\title{
THE GESNERIACEAE OF GUIANA
}

\section{A CRITICAL REVISION WITH NOTES ON SPECIES FROM ADJACENT REGIONS}

\author{
A. J. M. LEEUWENBERG ${ }^{1}$ ) \\ (Botanical Museum \& Herbarium, Utrecht)
}

(received March 31st, 1958)

\begin{abstract}
CONTENTS
CHAPTER 1. Introduction ................. 291

CHAPTER 2. Critical remarks and descriptions of new species. . . . 293

CHAPTER 3. Notes on geographical distribution and ecology . . . . . 323

GHAPTER 4. Revision of Chrysothemis . . . . . . . . . . . . 329

CHAPter 5. Revision of Napeanthus . . . . . . . . . . 340

CHAPTER 6. Revision of the Guianan Gesneriaceae . . . . . . . . 355

IITERATURE . . . . . . . . . . . . . . . . . 433

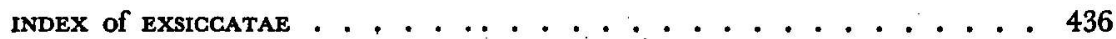

\section{CHAPTER 1}

\section{INTRODUGTION}

The present study was started as a revision of the Gesneriaceae of Suriname (Dutch Guiana). As it proved to be impossible to solve the taxonomic problems on the base of the scanty material from that country only and as the region of the three Guianas inclusive of the Brazilian territory "Amapá" and the Venezuelan "Delta Amacuro" turned out to be a sufficiently natural floristic unit, the author decided to extend his investigations to all Gesneriaceae known from that area.

Of those species material of many herbaria was studied in order to obtain a more complete impression of individual variation and geographical distribution. Among the material borrowed for this purpose the author came across misidentified (and unnamed) specimens some of which belonged to undescribed species. When the material was sufficiently ample and well-preserved new descriptions were drawn up; in some cases new combinations (transfers to other genera) were necessary. For the genera Chrysothemis and Napeanthus complete revisions were made, because otherwise it would have been impossible to work out properly the taxonomy of the Guiana species.

1) Assistant, Laboratorium voor Plantensystematiek en -geographie, Wageningen, Netherlands. 
The study was mainly carried out on material from the following herbaria:

A The Arnold Arboretum of Harvard University, Cambridge, Mass.

AWH Botanisch Museum Dr. H. van Heurck, Antwerpen, Belgium.

BM British Museum (Natural History), London, England.

BR Jardin Botanique de l'Etat, Bruxelles, Belgium.

C Botanical Museum \& Herbarium, Copenhagen, Denmark.

GGE Botany School, University of Cambridge, Cambridge, England.

F Chicago Natural History Museum, Chicago, Ill.

FI Herbarium Universitatis Florentinae, Florence, Italy.

G Herbarium Delessert, Conservatoire et Jardin Botaniques, Genève, Switzerland.

G-DC Herbarium of Prodromus of De Candolle, Conservatoire et Jardin Botaniques, Genève, Switzerland.

GH The Gray Herbarium of Harvard University, Cambridge, Mass.

GOET Systematisch-Geobotanisches Institut, Göttingen, Germany.

HBG Staatsinstitut für allgemeine Botanik und Botanischer Garten, Hamburg,

K The Herbarium, Royal Botanic Gardens, Kew, England.

L Rijksherbarium, Leiden, Netherlands.

LE Botanical Institute of the Academy of Sciences, Leningrad, U.S.S.R.

LINN The Linnean Society of London, London, England.

M Botanische Staatssammlung, München, Germany.

MA Instituto "Antonio José Cavanilles", Madrid, Spain.

MO The Missouri Botanical Garden, St. Louis, Mo.

NY The New York Botanical Garden, New York, N.Y.

P Herbier Général, Laboratoire de Phanérogamie, Muséum National d'Histoire Naturelle, Paris, France.

Separate collections at the Laboratoire de Phanérogamie at Paris:

P-BA Herbarium Baillon.

P-BO Herbarium Bonpland.

P-JU Herbarium Jussieu.

P-LA Herbarium Lamarck.

P-SU Herbarium Surian.

PH Academy of Natural Sciences, Philadelphia, Pennsylvania.

RO Istituto Botanico, Città Universitaria, Roma, Italy.

S Botanical Department, Naturhistoriska Riksmuseum, Stockholm, Sweden.

U Botanisch Museum \& Herbarium, Utrecht, Netherlands.

UC Herbarium of the University of California, Berkeley, California.

US United States National Herbarium, Smithsonian Institution, Washington, D.C.

W Naturhistorisches Museum, Wien, Austria.

WAG Laboratorium voor Plantensystematiek en -geographie, Wageningen, Netherlands.

The author is greatly indebted to the directors and curators of the herbaria who generously put their material at his disposal. Furthermore, he wishes to express his gratitude to DR. G. M. Schulze, Berlin-Dahlem, for sending him photographs of specimens in the Willdenow-Herbarium that could not be sent on loan.

In a few species living material from botanical gardens was available. They served principally for determining such characters as flowercolour, shape of corollas, habit of plants, etc., which are less readily observed in dried specimens. In some cases pickled specimens could be studied.

For locating the collecting localities the "Index to Map of Hispanic America" (Washington, 1943-'44) published by the American Geo- 
graphic Society proved to be of great help. The spelling of the names in this index has largely been followed. The maps are from Goode's series of base maps, published by the University of Chicago. Press.

\section{GHAPTER 2}

\section{CRITICAL REMARKS AND NEW SPECIES}

\section{NOTES ON MORPHOLOGY}

In drying the sappy stem $\mathbf{s}$ and leaves shrink, the stems become often sulcate and the leaves chartaceous or papyraceous. As flowers of dried specimens are fixed in one stage and as it is important to know what changes may take place in the bud during its development and during anthesis, flowers of cultivated plants and of herbarium specimens in various stages of development were studied. The zygomorphy of the flowers becomes more pronounced as the buds increase in size. TRAPP's observations (1956) on the development of stamens have been confirmed by the present author's investigations. The stamens curve towards each other shortly before the opening of the bud, putting the anthers in a connivent position often followed by coherence. In many species the filaments become contorted after the shedding of the pollen, as described by MOORE (1955, p. 111); by this the anthers often get detached from each other again. Coiled stamens were observed by the writer in living plants of Kohleria amabilis, Sinningia hybrids, Smithiantha hybrids, and Streptocarbus sp.; the phenomenon was found to be lacking in Columnea, gloriosa and Rechsteineria cardinalis. As the filaments become contorted, the stigma reaches maturity: the flowers are proterandric, as can readily be observed in cultivated Gesneriaceae and often be deduced in dried flowers from the stages of development of the organs involved in the phenomenon.

\section{RELATIONSHIP OF THE GENERA}

Some of the genera of the neotropical Gesneriaceae can be distinguished and defined with difficulty only, and there is great diversity of opinion as to the limits. There are several cases of close interrelationship of species usually placed in separate genera, rendering the boundaries between those genera vague or even untenable. Judging from these cases alone one would be compelled to unite large groups of genera or even complete tribes under one genus; this is particularly true for the Columneinae. The typical species of the genera are however so different that such a procedure would be quite undesirable. But part of these generic limits are based rather on convenient characters than on conspicuous discontinuities of features.

A consequence of this state of affairs is the presence of what may be called "borderline species", i.e. species that have some characters 
of one and some of an other genus. Nevertheless they can be assigned to one of the genera as certain characters may be considered as more important than others.

The author's views on the affinities of the critical genera involved in the present study are summed up in the chart which is presented below. The "points of contact" between the various genera have been numbered and comments on these are given in the following preceded by the corresponding number.

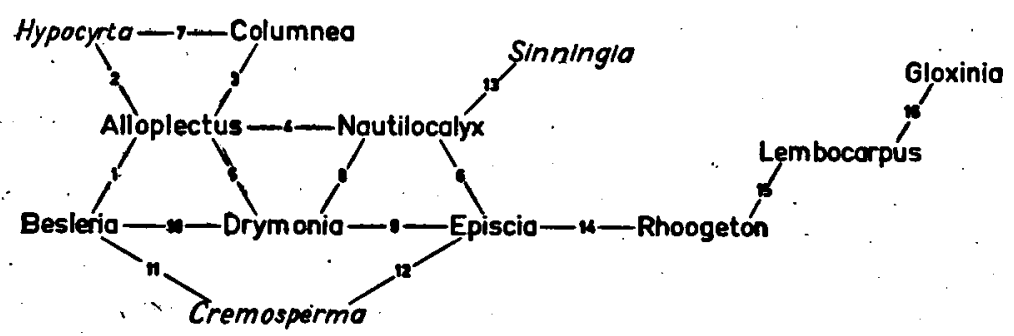

Chart of points of relationship between the critical genera involved in the present study. Those in italics do not occur in Guiana.

1. Alloplectus and Besleria differ mainly in the following points: Alloplectus

Corolla mostly hairy outside; Corolla often glabrous outside; sepals usually large; inflorescence when several-flowered with bracts; anther cells parallel, not confluent; fruit a capsule; disk of 1-5 glands.

Besleria

sepals smaller; inflorescence without bracts; anthers usually (at least in Guianan spp.) reniform, cells confluent at the apex; fruit a berry; disk mostly annular.

Points of similarity are habit and shape of the corolla. A "borderline species" is Alloplectus pycnosuzygius (Donn. Sm.) Morton as stipulated by Morton (1953, p. 522).

2. Alloplectus and Hypocyrta are closely related by their habit and their corolla, especially the limb; they can be separated as follows:

\section{Alloplectus}

Corolla mostly contracted in the throat, but not inflated; sepals usually $2 \times$ as long as wide or less.

\section{Hypocyrta}

Corolla strongly contracted in the throat, conspicuously inflated; sepals usually about $3-5 \times$ as long as wide.

3. Alloplectus and Columnea are very closely related. As far as represented in Guiana this is particularly the case with Alloplectus section Alloplectus and Columnea sections Collandra and Stygnanthe, through the shape of the corolla. They differ as follows: 
Alloplectus sect. Alloplectus

Leaves of a pair usually subequal, probably commonly decussate; fruit a capsule.

A. sect. Alloplectus

Sepals mostly less than $2 \times$ as long as wide; corolla limb actinomorphic or nearly so; fruit. a capsule, often fleshy.
Columnea sect. Collandra

Leaves of a pair strongly unequal, placed in one plane (in two ranks); fruit a berry.

C. sect. Stygnanthe

Sepals usually about $4 \times$ as long as wide; corolla limb obscurely bilabiate, the two dorsal lobes concave; fruit a berry, indehiscent.

A "borderline species" is Columnea lehmannii Mansf., as it has the calyx of Alloplectus. It is placed in Columnea because of its unequal leaves. When the fruits of more species will be known, more can be said about the boundary between these genera. At present it is not possible to give differential characters for both genera as a unit.

4. Alloplectus, especially section Heintzia and Nautilocalyx are very closely related by the inflorescence, capsule, and the shape of the corolla and often the sepals, but they differ mainly as follows:

\section{Alloplectus \\ Nautilocalyx}

Usually subshrubs or vines, if not corolla conspicuously contracted in the throat; sepals often large in proportion to the corolla.

Creeping or decumbent herbs; corolla not or hardly contracted in the throat, mostly about $3 \times$ as long as the calyx.

These genera are so closely related that it is hardly possible to give evident differential characters. In this case convenience, based on the above-named characters, indicates the limit rather than an exact definition. A "borderline species" is Nautilocalyx pallidus (Sprague) Sprague which was placed in this genus chiefly because of its habit and length of its corolla.

5. Alloplectus and Drymonia are closely allied by their habit, capsule, large sepals, and often sagittate anthers. The differential characters are:

\section{Alloplectus}

Corolla usually nearly cylindric and contracted in the throat; if not or hardly so, inflorescence with large bracts; sepals often coloured; corolla limb often small, actinomorphous or nearly so.
Drymonia

Corolla usually gradually widened, not contracted in the throat; sepals often green; corolla limb large, often slightly bilabiate.

6. Nautilocalyx is very close to Episcia sections Skiophila and Paradrymonia, the limit being determined by convenience rather than by exact definition as in Alloplectus. They differ as follows: 
Nautilocalyx

Inflorescence pedunculate and sepals covered by large bracts or inflorescence sessile; anther cells not confluent.
E piscia sect. Skiophila

Peduncle very short to conspicuous; pedicels elongate; sepals not covered by the bracts; bracts small; anther cells sometimes confluent at the apex.

A "borderline species" is Episcia mimuloides Benth. It is placed in Episcia as its anther cells are sometimes confluent.

Nautilocalyx

Stems usually longer than leaves; sepals mostly less than $5 \times$ as long as wide; anther cells glabrous.

\section{E. sect. Paradrymonia}

Stems usually shorter than leaves; sepals linear, about $10 \times$ as long as wide; anther cells often with stiff bristles.

7. Columnea, at leastı $\mathrm{n}$ some of its sections, is related to Hypocyrta by the habit and the calyx. These genera differ mainly by the following characters:

Columnea

Corolla not inflated, subactinomorphous to strongly bilabiate, not or hardly contracted in the throat; fruit a berry. . .

\section{Hypocyrta}

Corolla inflated, conspicuously contracted in the throat; fruit a capsule.

8. Nautilocalyx is allied to Drymonia, though less closely than to Alloplectus; the two genera differ in the following characters:

Nautilocalyx

Sepals usually comparatively small; corolla nearly cylindric or infundibuliform, mostly about 3 $X$ as long as the calyx; anthers orbicular or nearly so, not sagittate.

Points of similarity are the capsule and often the leaves and the calyx.

9. Drymonia and Episcia are related by the capsule and often by the shape of the corolla. The main differences are:

Drymonia

Usually subshrubs or vines; sepals mostly large in proportion to the corolla; corolla usually widely infundibuliform; anthers often sagittate at the base.
Drymonia

Sepals large; corolla widely infundibuliform, mostly $1-2 \times$ as long as the calyx; anthers mostly oblong, often sagittate at the base.

10. Besleria and Drymonia resemble each other by their habit and sometimes by their inflorescences and flowers, but differ much by the following characters: 


\section{Besleria}

Sepals entire, rather small; corolla often cylindric; anthers reniform, cells confluent at the apex; no bracts; fruit a berry.

\section{Drymonia}

Sepals often toothed, large; corolla mostly amply infundibuliform; anthers oblong, often sagittate at the base, cells parallel; inflorescence often with bracts; fruit a capsule.

11. Besleria is most closely related to Cremosperma as stipulated by MoRTon who also pointed out the differentiating characters (1939, p. 402).

12. Episcia, especially section Trichosperma Lwbg. (p. 312) is related to Cremosperma by the habit and the anthers with confluent cells. The principal differences are:

\section{E. sect. Trichosperma}

Peduncle obsolete; flowers in axillary fascicules; sepals free; disk of one dorsal gland.

\section{Cremosperma}

Peduncle conspicuous; inflorescence umbellate or capitate; sepals united; disk often annular.

13. Nautilocalyx is allied to Sinningia, especially to $S$. villosa which has a superior ovary, by the habit and the flowers. Both genera differ in the following characters:

Nautilocalyx

No tubers; sometimes creeping with adventitious roots; ovary superior; sepals free or nearly so.

\section{Sinningia}

Tubers; not creeping; ovary mostly partially inferior; if superior sepals united.

14. Rhoogeton Lwbg. is related to Episcia section Episcia as pointed out on p. 322.

15. Lembocarpus Lwbg. is allied to Rhoogeton as stipulated on p. 322.

16. Lembocarpus is distantly related to Gloxinia by the shape of the corolla, but differs conspicuously in the following characters:

Lembocarpus

Tubers; acaulescent; inflorescence conspicuously pedunculate; ovary superior.

\section{Gloxinia}

Rhizomes; caulescent; flowers axillary, mostly solitary; ovary inferior.

Cenera with critical and NeW Species

Alloplectus Mart., Nov. Gen. 3: 53.1829.

This genus is related to five other genera as pointed out in the numbers $1-5$ of the above chart. In Guiana it is represented by the following two sections: Alloplectus with 3 species, $A$. cristatus, A. meridensis(?) and A. savannarum, and Macrochlamys (Dcne.) Fritsch with $A$. coccineus. 
A. cristatus (L.) Mart. ex G. Don, Gen. Syst. 4: 655. 1838 (see also Martius, Nov. Gen. 3: 57. 1829); D.C., Prod. 7: 545. 1839; Grisebach, Fl. Br. W.I. Isl. 463. 1861 (“1864"); Hanstein, Linnaea 34: 375. 1865; Duss, Fl. Ant. fr. 432. 1897; Urban, Symb. Ant. 2: 356. 1901; in Fedde, Repert. Beih. 5: 47. 1920; Morton, Contrib. U.S. Nat. Herb. 29: 17. 1944.

Basionym: Besleria cristata L., Sp. Pl. 619. 1753; Jacquin, Sel. Stirp. Amer. Hist. 188, t. 119. 1763.

Neotype: Burmann, Plant. Amer. Car. Plumier. 1756. t. 50, "Besleria pedunculis simplicibus solitariis".

For comments on typification see under Besleria, p. 300. Homotypic synonyms: Crantzia cristata (L.) Scop., Intr. 173. 1777; Fritsch in Engl. Prantl, Nat. Pflanzenf. 4 (3b) : 168. 1894 ("1895"). Prionoplectus cristatus (L.) Oerst., Centralamer. Gesn. 78, t. 9. f. 36-40. 1858.

Lophia phoenicea Desv. ex Hamilton, Prod. Pl. Ind. Occ. 47. 1825.

$A$. cristatus is known from the Lesser Antilles in which region three varieties were distinguished by MorTon (1944, p. 17). The Guianan material of this species belongs to a new variety that may be keyed out from the others as follows:

1. Sepals laciniate and serrate. Martinique, St. Lucia. . . . . . . . . . . . . . A. c. var. cristatus Sepals simply incised, serrate or crenate . . . . . . 2

2. Sepals crenate, obtuse. Grenada, St. Vincent. . . . . . . . . . . . . . . . . . . . A. c. var. crenatus Sepals serrate or dentate-serrate, acute or acuminate . . . 3

3. Sepals sharply dentate-serrate; stems and pedicels hirsutulous. British Guiana . . . . . . A. c. var. epirotes

Sepals serrate; stems and pedicels densely strigose or subtomentose. Lesser Antilles north of Martinique .. . . . .................. c. var. brevicalyx

A. cristatus var. epirotes Lwbg., var. nov.

Caulis, petioli et folia subtus hirsutuli. Lamina apice paulum acuminata minutius crenato-serrata. Sepala acriter dentato-serrata, acuta.

Type: British Guiana: Mt. Ayanganna, Pakaraima Mts., Maguire, Bagshaw \& Maguire 40594. (NY, holotype; isotypes: U, US).

Leaf blade $2-3 \times$ as long as wide, $5-10 \times 2-4.5 \mathrm{~cm}$. Pedicel red, 5-8 cm long. Sepals about $22 \times 20 \mathrm{~mm}$.

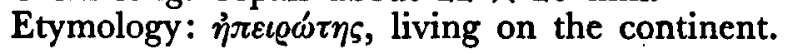

All West Indian specimens of $A$. cristatus examined could be identified to the variety. Enumeration of representative specimens:

A.c. var. cristatus:

MARTinique: Belanger 176(P), 194(P, G); Forsström s.n.(S); Hahn 118 (P, BM, G, K, L, U, UC); Mouret 219 (P), s.n. (L); Mme. Rivoire, anno 1839 (P); Sieber 161 (BR, K, L, M, MO, P); herb. Sieber s.n. (AWH); Terrasson 39 (P); herb. 
de Ventenat 440 (G); Plée, May 1820 (P); Rodriguez 3363 (P, U); Privault 113 (P); Stehlé $2133(\mathrm{P})$; Trinité, herb. Sieber s.n. (AWH).

ST. Lucia: Box 1725 (BM).

A.c. var. brevicalyx Morton, Contrib. U.S. Nat. Herb. 29: 17. 1944:

St. BarthelemY: Forsström, herb. Swartz s.n. (S).

St. Krrrs: Fairchild, 24 Jan. 1932 (BM); Forsström, herb. Swartz s.n. (S); Britton \& Cowell 300 (K, paratype).

Nevis: Jobin s.n. (G).

MontSerRat: Hollings, 30 April 1888 (K).

GUADELOUPE: Duss 2985 :(F, paratype); Duchassaing s.n. (GOET); Funck \& Schlim 38 (BM, BR, G, P, U, W); Quentin 485 (P), 700 (P).

Dominica: Hoskin 100 (CGE); Imray 75 (K), 152 (K); Laudat, Lloyd 191 (K, isotype); Othmer, 30 Oct. 1903 (M); Ramage, 27 Mar. 1888 (K), 6 Apr. 1888 (K), 24 Apr. 1888 (K); Eggers 602 (BR, G, GOET, L, M, P, U, UC, paratypes); Hodge 825 (BM, paratype).

A.c. var. crenatus Morton, l.c. p. 17:

Grenada: G. N. Smith 72 (K); Sherring 267 (F, paratype), s.n. (K); Eggers 6160 (L, P, paratypes); Broadway, Mar. 1906 (K); Grand Etang, Broadway 3719 (L), 4526 (BR).

ST. VINCENT: Anderson s.n. (K); Guildingh s.n. (K); herb. Sieber 20 (AWH); Beard 1428 (S); Parker s.n. (K).

A. coccineus (Aubl.) Mart. ex G. Don, Gen. Syst. 4: 655. 1838 (see also Martius, Nov. Gen. 3: 57. 1829); D.C., Prod. 7: 545. 1839; Hanstein, Linnaea 34: 366. 1865; Lemée, Fl. Guian. fr. 3: 463. 1953.

Basionym: Besleria coccinea Aubl., Hist. Pl. Guian. 2: 632. 1775.

Type: French Guiana: banks of Sinnemary R., 20 miles from the sea coast, Aublet s.n. (BM).

Homotypic synonym: Crantzia coccinea (Aubl.) Fritsch in Engl. Prantl, Nat. Pflanzenf. 4 (3b): 168. 1894 ("1895").

Heterotypic synonyms: Alloplectus circinatus Mart., Nov. Gen. 3: 56, t. 223. f. 2. 1829; G. Don, Gen. Syst. 4: 655. 1838; D.C., Prod. 7: 545. 1839; Hanstein, Linnaea 26: 177, 209, 215, t. 2. f. 50A. 1853; in Martius, Fl. Bras. 8 (1): 406. 1864; Linnaea 34: 366.1865. Lectotype: BRAzIL: Amazonas, near Madeira R., Martius herb. 75 (M, photographs: U, US).

Alloplectus patrisii D.C., Prod. 7: 545. 1839; Bentham, Lond. Journ. Bot. 5: 362. 1846; Schomburgk, Reisen Br. Guian. 3: 972. 1848; Hanstein, Linnaea 34: 365. 1865; Lemée, F1. Guian. fr. 3: 463. 1953. Type: French Gulana: Cayenne, Patris s.n. (G-DC, 4 sheets [one number?], , photographs of first: F, GH, US).

Homotypic synonym: Crantzia patrisii (D.C.) Fritsch in Engl. Prantl, Nat. Pflanzenf. 4 (3b): 168. 1894 ("1895"); in Engl., Bot. Jahrb. 37: 486. 1906; Pulle, Enum. 420. 1906.

This species was correctly described by Aublet, but his drawing was faulty; consequently interpretation of the name was only possible by study of the type. Its characters agree with those of $A$. patrisii and of $A$. circinatus. When the inflorescence develops and the corollas of the first flowers are shed it loses the outer bracts as figured by Martius (1829, t. 223. f. 2).

A. coccineus can be devided into two varieties as follows: 
Corolla yellow, usually not spotted. Basal part of peduncle below the bracts very short, much shorter than the outer bracts, mostly about one-fifth of the length (from 0.11 to 0.36 ) of the outer bracts.

Distribution: British Guiana, Suriname (only known from most eastern part), French Guiana, northern and central Brazil.

Extra-Guianan specimens:

Brazil: Amazonas: Rio Vaupés, near Panure, Spruce 2604 (K, P); Rio Jurua, Bom Fim, Ule 5215 (G, HBG); near mouth of R. Madeira, Martius herb. 77 (M, paratype of $A$. circinatus); near R. Madeira, Martius herb. 75 (M, lectotype of A. circinatus); "Prov. Rio Negro", Martius herb. 76 (M, paratype of A, circinatus).

GuAPORE: Upper Machado Region, source of R. Jatuarana, Krukoff 1526

(NY, G, K, P, U); ibid., near Tabajara, Krukoff 1353 (NY, G, K, P, U).

PARÁ: Ilha de Marajó, Spruce 1850 (K); Belem, Dahlgren \& Sella 520 (F); vic. of Belem, Baker 99 (BM, G, P, U) ; Upper R. Cupary, plateau between R. Xingu and R. Tapajos, Krukoff 1217 (NY, BM, G, K, P, S, U); Tapajos Region, Aramanahy, Monteiro da Costa 225 (F).

PIauf: Martius herb. 73 and 74 (paratypes of A. circinatus); near R. Parnaiba, Martius herb. 78.(M, paratype of A , circinatis).

Alagoas?: Sertão, von Wied, March 1817 (BR).

BAHIA: Blanchet 1465 (BM), 1835 (G), 2121 (BM), 2122 (P), s.n. (BM); Ilheos, Blanchet 1839 (G).

Sin. loc.: herb. J. E. Smith 1058. 3 (LINN).

A. coccineus var. fusco-maculatus Lwbg., var. nov. Fig. 8, p. 362

Corolla ochroleuca, flava vel alba, fauce vel limbo fusco-purpureovel rubro-fusco-maculata. Pars basalis pedunculi sub bracteis bracteas exteriores longitudine fere aequans.

Type: Suriname: Nassau Mts., Cowan \& Lindeman 39049 (U). Peduncle about 1.2 (from $0.4-2.2$ ) $\times$ as long as the outer bracts. Distribution: Suriname.

Paratypes see p. 361 .

The only specimen with spotted corollas and short peduncles was collected by Ule (5215) in Brazil.

Besleria L., Sp. Pl. 619. 1753.

This genus, one of the largest of the New World Gesneriaceae, is related to Alloplectus, Drymonia, and Cremosperma as pointed out under nos. 1, 10, and 11 of the preceding paragraph. All seven Guianan species have been dealt with by Morton (1939), one of them, $B$. flavo-virens, being of doubtful identity. For the arrangement of the species in sections and subsections the reader is referred to Morton's revision (l.c.).

LiNNAEUs described three species of this genus which were all taken from Plumier's "Nova Plantarum Americanarum Genera" (1703), namely $B$. [Episcia] melittifolia, $B$. lutea and $B$. [Alloplectus] cristata. Of the second species two varieties are distinguished which had been treated as species by Plumier. This author who left no herbarium depicted several flowers of Besleria, unfortunately not very clearly and without indication of species. The plates are therefore not eligible as types. BuRManN published later (after 1753) more elaborate draw- 
ings by Plumier which agree with our present-day concepts of the species. There are no specimens in the Linnean Herbarium and therefore BuRMANN's plates are designated by the present author as neotypes:

Burmann, Plant. Amer. Car. Plumier. etc. 1756. t. 48, "Besleria pedunculis ramosis", neotype of Episcia melittifolia (L.) Mart.

Burmann, op. cit. t. 49. f. 1, "Besleria pedunculis simplicibus confertis", neotype of Besleria lutea L.

Burmann, op. cit. t. 50, "Besleria pedunculis simplicibus solitariis", neotype of Alloplectus cristatus (L.) Mart. ex G. Don.

Of the three species of Besleria mentioned above two were subsequently removed to the genera indicated in brackets and the third, $B$. lutea, becomes automatically the type species of the genus as stated by Hitchcock and GreeN (1929, p. 167).

B. flavo-virens Nees et Mart., Nov. Act. Acad. Leop. Carol. 11 : 49. 1823; D.G., Prod. 7: 538. 1839; Hanstein in Martius, Fl. Bras. 8 (1): 400, t. 65. f. 6. 1864; Linnaea 34: 332. 1865; Fritsch in Engl., Bot. Jahrb. 37: 483. 1906; Morton, Contrib. U.S. Nat. Herb. 26: 473. 1939.

Fig. 10, p. 367

Type: Brazil: Bahia, Ilheos, Felisberta, vic. of São Pedro de Alcantara, von Wied, anno 1816 (BR, 2 sheets, photographs of one in $U$ and US).

Homotypic synonym: Besleria luteo-virens Mart., Nov. Gen. 3: 45. 1829; G. Don, Gen. Syst. 4: 651. 1838.

As this species has corollas with a subactinomorphic terminal limb and without spur it belongs to the section Besleria. Its peduncle would place it in the subsections Pedunculatae or Elongatae, but by the size of the calyx it would go in the Elongatae, because that is not less than half as long as the corolla; on the other hand its shape is characteristic for the Pedunculatae. Its closest relative is $B$. minutiflora from which it differs mainly by the way of branching of the inflorescence. The points of similarity are the size of the flowers, the white corolla, the rounded sepals, the habit, and the leaves. In Morton's key (1939, p. 410) B. flavo-virens is keyed out to the heading: "Corolla white, 5-6 mm long" (disregarding the number of primary veins) and can be distinguished from $B$. minutiflora as follows:

Inflorescence compound-subumbellate, not congested; corolla not ventricose. PERU . . . . . . . . . B. minutiflora

Inflorescence congested-paniculate; corolla dorsally bag-shaped. Guiana, Brazil • • • • . . . . . . B. flavo-virens

The holotype of $B$. minutiflora has been destroyed in the Berlin herbarium. An isotype is in HBG!

Extra-Guianan specimens are:

BraziL: BaHIA: Blanchet 1857 (G); Ilheos, Felisberta, vic. of São Pedro de Alcantara, von Wied, anno 1816 (BR, type); BAHIA?: Castelnova, Riedel 419 (K, US).

Sin. Loc., perhaps French Gutana: Herb. J. E. Smith 1058. 2 (LINN). 
Codonanthe (Mart.) Hanst., Linnaea 26: 209. 1853.

The two species of this genus which occur in Guiana are maintained as separate following SANDwITH (1931, p. 490) and MorTon (1935, p. $1159 ; 1948$, p. 564$)$.

C. crassifolia (Focke) Morton, Field Mus. Publ. Bot. 18: 1159. 1938; Bull. Torr. Bot. Cl. 75: 564. 1948; in Fl. Trin. and Tob. 2 (5): 307. 1955; Fieldiana 28: 1092. 1957; Lemée, Fl. Guian. fr. 3: 461. 1953. Fig. 13 Cr, p. 379

Basionym: Hypocyrta crassifolia Focke, Tijdschr. Nat. Wetensch. 5: 199. 1852.

Type: Suriname: Focke, coloured pencil drawing (L, photographs: U, WAG).

Heterotypic synonyms: Codonanthe confusa Sandwith, Kew Bull. 1931 : 492. Type: French Guiana: Acarouany R., left bank Lower Mana R., Sagot 426 (K, holotype, isotypes: BM, BR, P, 4 sheets, U, W, photographs of one $P$ sheet in U and US).

Misapplied name: Codonanthe calcarata Hanst., Linnaea 34: 416. 1865, exclusive of type.

Episcia hookeri Hanst., Linnaea 34: 350. 1865; Morton in Fl. Trin. and Tob. 2 (5): 309. 1955. Type: Hooker, Icones 9: 873. 1852, no specimens preserved.

Misapplied name: Centrosolenia glabra Hook., Icon. cit., not Benth.

In accordance with MORTON's opinion Episcia hookeri is regarded as a synonym of Codonanthe crassifolia, although the type-plate shows an aberrant specimen. Only one specimen of $C$. crassifolia collected by Broadway in Trinidad has some serrulate leaves as figured by Hooker.

Specimens from outside Guiana are:

British Honduras: Sibun R., 'Gentle 1693 (GH, K, MO); Stann Ck. distr., Gentle $2993(\mathrm{GH})$.

Guatemala: Petén: along R. San Diego, Steyermark 45412 (A, MO).

Honduras: Atrántida: Lancetilla Valley, Standley 52699 (A); vic. of La Ceiba, Yuncker, Koepper \& Wagner 8001 (BM, G, GH, K, MO, S).

Costa Rica: vic. of Puerto Limón, Cufodontis 721 (W); Lmón: Dodge \& Goerger 9596 (MO).

Panama: Bocas del Toro: vic. of Chiriquí Lagoon, von Wedel 1156 (MO, GH), 1296 (MO, GH), 1875 (MO, GH), 2549 (MO, GH), 2782 (MO, GH), 2893 (MO, GH); C.Z.: Barro Colorado Island, Bangham 604 (A); Chagres, Fendler $178(\mathrm{~K})$.

Colombia: Jiménez, R. Dagua, André 2484 (K); El Valle: Buenaventura, Killip 5221 (NY).

Venezuela: Bolfvar: between Ptari-tepuí and Soropán-tepuí, Steyermark 60732 (F); Chimantá Massif, Steyermark 75390 (US).

Trinidad: Barneu, Caroni, Britton \& Mendelson 823 (NY); Maracas Valley, Britton \& Hazen 1633 (NY, K); Blanchisseuse Road, Broadway, 25 Oct. 1931 (BM); Toco Road, Valencia, Britton, Hazen \& Mendelson 1784 (NY, GH); vic. of Valencia, Britton \& Britton 2835 (NY); Oropuche Road, Broadway 6175 (BM, K, S), 6926 (BM); Aripo Road, Broadway, 12 June 1925 (K); Ortoire R., Guayguayare Road, Britton, Freeman \& Nowell 2541 (NY); Quarc R., Broadway 7486 (BM), 11 May 1928 (K); St. Anns, Jan (?) des Amandes, Broadway, 9 May 1930 (BM); Caura, Broadway, 24 June 1934 (K); Santa Cruz, herb. Trin. 7008 (K); Mt. Jamana, Broadway 7974 (BM); Windbelt Reserve, herb. Trin. 15059 (K); Broadway 6784 (K); Fendler 490 (K). 
Columnea L., Sp. P1. 638. 1753.

This genus is perhaps the largest of the New World Gesneriaceae. It is closely related to Alloplectus and Hypocyrta as discussed in the numbers 3 and 7 of the preceding paragraph. In Guiana it is represented by 5 species in 3 sections: section Columnea with two species, C. scandens and $C$ : oerstediana, section Collandra (Lem.) Benth. et Hook. with two, C. aureonitens(?) and C. guianensis, and section Stygnanthe (Hanst.) Benth. et Hook, with one species, C. calotricha.

C. scandens L., Sp. P1. 638. 1753; Jacquin, Hort. Vindob. 27, t. 48. 1776; Sims, Bot. Mag. 39: t. 1614. 1814; Loddiges, Bot. Cab. 5: t. 403. 1820; Edwards, Bot. Reg. 10: t. 805. 1824; Martius, Nov. Gen. 3: 65, t. 226. f. 2. 1829; G. Don, Gen. Syst. 4: 652. 1838; D.C., Prod. 7: 542. 1839; Hanstein, Linnaea 26: 187, 211. 1853; Hooker, Bot. Mag. 85: t. 5118. 1859; Grisebach, Fl. Br. W.I. Isl. 464. 1861 ("1864"); Houllet, Rev. Hortic. 1864: pl. 231; Hanstein, Linnaea 34: 404. 1865; Duss, Fl. Ant. fr. 432. 1897; Urban, Symb. Ant. 2: 360. 1901; Morton, Fieldiana 28: 531. 1953; in Fl. Trin. and Tob. 2 (5): 312. 1955.

Neotype: Burmann, Plant. Amer. Car. Plumier. 1756. t. 139. f. 1, "Columnea".

Homotypic synonym: Glycanthes scandens (L.) Raf., Sylva Tellur. 83. 1838.

Heterotypic synonyms: Columnea rotundifolia Salisb., Parad. Lond. 1 (1): t. 29. 1806; Hanstein, Linnaea 34: 404. 1865; Urban, Symb. Ant. 2: 360. 1901. Type: TrINIDAD: Salisbury, icon. cit. (no specimen preserved).

Columnea jacquiniana Presl, Abh. Böhm. Ges. Wiss. 5 (3): 576.1845. Type: Jacquin, Hort. Vindob. t. 48. 1776 (specimen after which it might have been depicted: Herb. Jacquin, from Martinique (BM).). Columnea speciosa Presl, 1.c. p. 575; Grisebach, Fl. Br. W.I. Isl. 464. 1861 ("1864"). Type not seen (mentioned as: "cult. in hortis").

Columnea fendleri Sprague, Kew Bull. 1912: 41; Morton, Fieldiana 28: 529. 1953. Type: Venezuela: Mérida?. near Tovar, Fendler 2031 (K, holotype, isotype: GOET).

Columnea aripoensis Britton, Bull. Torr. Bot. Cl. 53: 470.1926.

Type: Trinidad: Heights of Aripo, Britton \& Freeman 2340 (NY). Homotypic synonym: C. scandens var. aripoensis (Britton) Morton, Contrib. U.S. Nat. Herb. 29: 14. 1944; in Fl. Trin. and Tob. 2 (5): 313. 1955.

C. scandens var. vincentina Morton, Contrib. U.S. Nat. Herb. 29: 13. 1944. Type: St. Vincent: Mt. St. Andrews, Eggers 6698 (US, holotype, isotype: $\mathrm{P}$ ).

C. scandens var. australis Morton, Fieldiana 28: 531. 1953. Type: Venezuela: Monagas, Montaña de Aguacate, between Caripe and Garipito, Steyermark 62229 (F, holotype, isotype: US).

Linnaeus described one species of Columnea: $C$. scandens with two varieties. The first is the present $C$. scandens, the second is perhaps a colour-form of $C$. tulae Urb. These two varieties were treated by 
Plumiter ás twò species. As in Besleria he also depicted several flowers of Columnea without indication of species. On the same grounds as mentioned there (p. 300) the present author designates BurmanN's plate as neotype:

Burmann, Pl. Amer. Car. Plumier. etc. 1756. t. 139. f. 1, "Columnea".

In his manuscript which is preserved in the library of the Kew Herbarium, Plumier stated that Columnea scandens phoenicea flore fructo albo occurs in Martinique. The sepals on the plate in this manuscript have some teeth near the base; those on BurManN's plate are entire. Both plates agree with $C$. scandens var. scandens sensu MoRTon (1944, p. 13).

C. scandens is a very variable species of which four varieties have been distinguished by MORTON. However, in the present author's. opinion, these varieties and $G$. fendleri which was supposed to be a related species cannot be maintained as separate entities. The most important characters that served to distinguish these taxa lie in the sepals which show the following peculiarities in what may be called typical specimens:

C. fendleri: linear, 8-10 $\times$ as long as wide, subulate, with some teeth. Venezuela.

C. s. var. scandens: linear-lanceolate, $4-6 \times$ as long as wide, acuminate, with some small or without teeth. MartiniQue, MonTserrat.

C. s. var. aripoensis: oblong-lanceolate, $2.5-5 \times$ as long as wide, acute or acuminate, mostly without teeth. Trinidad; ToBAgo.

C. s. var. vincentina: ovate-lanceolate or triangular-lanceolate, 2.5-5 $\times$ as long as wide, acuminate, with several large teeth. ST. Vincent, GrenAda.

C. s. var. australis: lanceolate, $3-4 \times$ as long as wide, acuminate, with some teeth. VenEzuELA.

It turned out, however, that intermediates exist, all taxa grading into "typical" $C$. seandens and most of them also into one another. These intermediates also obscure the correlation between morphological characters and geographical distribution. An amended description of $C$. scandens taking into account its great variability in the extended circumscription may be given as follows:

Leaf blade elliptic or oblong-elliptic, 1.4-3.5 $\times$ as long as wide (in all forms), 15-65 $\times 9-38 \mathrm{~mm}$, acute or obtuse at the apex, cuneate or rounded at the base, entire, subentire, or remotely and shallowly crenate-dentate (in all forms), mostly strigose to glabrous or sometimes subtomentose (most specimens from Dominica, Venezuela, and Golombia) on both sides. Sepals ovate-lanceolate, lanceolate, or linear, 2.5-10 $\times$ as long as wide, acuminate, acute, or subulate at the apex, entire or with some teeth near the base, 8-17 $\times 1-4 \mathrm{~mm}$, strigose on both sides, especially outside. Corolla $4-6 \times$ as long as the calyx, 43-70 mm long, extending its length during anthesis!; tube 1-2 $\times$ as long as the galea. Ovary appressed-pubescent or glabrous (type of $C$. fendleri and at least some Dominican specimens). 
Representative specimens (except Guianan) are:

Montserrat: Shafer 489 (NY), 546 (NY, US).

GuAdeloupe: Gravry (?), Dec. 1844 (P).

Dominica: Eggers 716 (BR, G, GOET, L, M); Hoskin 50 (CGE); Imray 194 (K, GOET), s.n. (K); Mouret, March 1911 (L); Ramage, 3 Apr. 1888 (K); Hodge \& Hodge 1075 (NY).

MARTINIQUE: Mouret $218(\dot{\mathrm{P}})$; Duss 1876 (NY), 4637 (NY, US); Isert, anno 1787 (C), s.n. (C); herb. Jacquin s.n. (BM); Plée 471 (P); L. C. Richard s.n. (P); Mme. Rivoire, anno 1839 (P); Rodriguez 3609 (P), 3806 (P); herb. Sieber s.n. (AWH); Thiébaut 331 (P); Hahn 287 (P); Torfeeth s.n. (C); Ewan 17081 (US). ST. LuGra: Beard 1041 (US); Howard 11517 (US), 11674 (US).

ST. VINCENT: Anderson s.n. (K); Eggers 6698 (US, holotype of C.s. var. vincentina, isotype: P), 6199 (P); Guildingh s.n. (K, GOET); herb. Sieber 496 (AHW); Morton 5291 (US), 5532 (US), 6224 (US).

GrenADA: Beard 1241 (K, S, US); Broadway 5789 (BR); G. N. Smith 107 (K); Miller 127 (US); Morton 4618 (US).

Tobago: Sandwith 1837 (K); herb. Trin. $15298(\mathrm{~K})$.

TRINIDAD: Heights of Aripo, Broadway 9856 (K, NY, topotype of C.s. var. aripoensis); ibid., Herb. Trin. 14017 (Simmonds 226) (US); North Mt. Range, Graf 2 (NY); Ciparia, Herb. Trin. 2514 (US).

Venezuela: Monagas: Montaña de Aguacate, between Caripe and Caripito, Steyermark 62229 (F, holotype of C.s. var. australis, isotype US).

AnzoÁtegui: near El Limón, Pittier 13521 (F).

ARAGUa: P. N. Aragua, Ll. Williams 12230 (F); Rancho Grande, Ll. Williams $10052(\mathrm{~F})$.

MÉRIDA?: near Tovar 2031 (K, holotype of C. fendleri, isotype: GOET).

Colombia: Karsten s.n. (BM, G).

Drymonia Mart., Nov. Gen. 3: 57. 1829.

Drymonia is closely allied to Alloplectus and Episcia, and less so to Nautilocalyx and Besleria (nos. 5, 8, 9, and 10 of preceding paragraph). In Guiana it is represented by three species of which two belong to the section Drymonia (Genuinae Hanst.) and one described below that cannot at present be assigned to a section.

\section{D. campostyla Lwbg., sp. nov. Figs. 19, p. 394; 20, p. 395}

Herba suffrutescens epiphytica vel terrestris repens. Tota planta facie interiore corollae excepta pilis hyalinis circiter 6-cellularibus vestita. Folia opposita laminis ovato-oblongis. Inflorescentia axillaris 1-3-flora. Sepala libera foliacea apice longe acuminata. Corolla alba intus flava ample tubulosa fauce non contracta basi calcarata limbo patente. Stamina quattuor didynama inclusa filamentis contortis basi omnibus connatis et basi corollae usque ad 4-5 mm adnatis antheris omnibus cohaerentibus. Ovarium superum hirsutum. Stylus pilis saepe obscure aggregatis vestitus. Stigma bilobum. Disci glandula dorsalis singula. Placentae duae parietales bilamellatae utraque facie ovula creberrima gerentes. Fructus immaturus globosus.

Type: Suriname: Upper Suriname R., near Kabel, Jonker 625 (U).

Complete diagnose see p. 393.

Paratypes:

SURINAME: Abontjeman, Sara Ck., Suriname R., coll. indig. 279 (U); Emmaketen, B.W. 5642 (U).

FRENCH GuiANA: Marowijne R., Wachenheim 173 (P); ibid., vic. of Codebert, Wachenheim $162(\mathrm{P})$; Lower Mana R., Charvein, Benoist $497(\mathrm{P})$. 
Etymology: $x a ́ \mu \pi \eta$, caterpillar, the style having obscurely aggregated hairs like a caterpillar.

This species differs from most species of Drymonia by its longacuminate sepals and its anthers which are not sagittate, and coherent rather than connivent. With the data at hand it is not possible to say what species is its closest relative.

D. serrulata (Jacq.) Mart. ex D.C., Prod. 7: 543. 1839 (see also Martius, Nov. Gen. 3: 59. 1829); Hanstein, Linnaea 26: 179, 207. 1853; op. cit. 34: 354. 1865; Urban, Symb. Ant. 2: 355. 1901; Pulle, Enum. 420. 1906; Johnston, Fl. Isl. Margarita, Contrib. Gray Herb. 34 (7): 260. 1909; Sandwith, Kew Bull. 1938: 371; Morton in Fl. Trin. and Tob. 2 (5): 309. 1955.

Fig. 21, p. 396 1798.

Basionym: Besleria serrulata Jacq., Hort. Schönbr. 3: 21, t. 290.

Type: Cult. in Hort. Schönbrunn, Jacquin s.n. (W, ster.).

Homotypic synonyms: Drymonia bicolor Lindl., Bot. Reg. 24: t. 4. 1838; Otto, Allg. Gartenz. 6: 158, t. 4. 1838.

Drymonia jacquini G. Don, Gen. Syst. 4: 653. 1838.

Heterotypic synonyms: Besleria spectabilis H.B.K., Nov. Gen. 2: 321. 1818 ("1817"). Type: Colombia: Cauca, Smitha R., between La Sequia and Popayan, Humboldt \& Bonpland 2028 (P-BO).

Homotypic synonym: Drymonia spectabilis (H.B.K.) Mart. ex G. Don, l.c. (see also Martius, l.c.); D.C., l.c.; Hanstein, Linnaea 26: 179. 1853; op. cit. 34: 355. 1865; Oersted, Centralamer. Gesn. 37. 1858; Morton, Field Mus. Publ. Bot. 18: 1175. 1938; Johnston, Sargentia 8: 276. 1949.

Drymonia cristata Miq., Linnaea 18: 26. 1844; Lemaire, Fl. d. Serres Sér. 1. 4: pl. 388. 1848; Lindley and Paxton, Flower Garden 1: 41, 42, f. 25. 1850; Hanstein, Linnaea 34: 356. 1865; Pulle, Enum. 420. 1906; Morton, Bull. Torr. Bot. Cl. 75: 565. 1948; Lemée, Fl. Guian. fr. 3: 460. 1953. Type: Suriname: Focke 766 (U, flowers lost).

Homotypic synonym: D. sarmentosula Lem., 1.c.

D. parvifolia Griseb., Fl. Br. W.I. Isl. 463. 1861 ("1864"); Urban, 1.c., not Morton. Type: Colombia: Magdalena, Santa Marta (not Jamaica!), Purdie 177 (GOET, holotype, isotype: K, photographs in $U$ and WAG).

D. maculata S. Moore, Trans. Linn. Soc. Ser. 2. 4: 410. 1895. Type: Brazil: Matto Grosso, Barro do Bugres, S. Moore 529 (BM).

The first description of this species made by JAcQuin is based on a cultivated specimen originally from Trinidad. Later it was described by KunTh from Colombia as Besleria spectabilis, by Miquel from Suriname as $D$. cristata, by GRIsEBACH from Colombia as $D$. parvifolia, and by Spencer Moore from Matto Grosso as D. maculata. After comparison of the types and more than a hundred specimens no character could be found to maintain any of these species. $D$. serrulata is a variable species, but there is no character that can be used for subdividing it into varieties, as the variable characters are rather 
continuously and independently variable. Most of the variation lies in the shape and the indument of the sepals and the colour of the corolla. The sepals are acute, acuminate, or sometimes obtuse at the apex, entire, sinuate, or serrulate, puberulous or sparsely appressedpubescent on both sides. The corolla is creamy, pale yellow, pinkish, pink, purple- or dark red-veined or -spotted, or purple. According to Johnston (no. 62) the corolla changes during anthesis from pink to purple. The difficulties about the interpretation of JAcQuiN's name arose probably as a consequence of the corolla being wrongly depicted on his plate, the ventral lobe being shown as serrate instead of erosefimbriate. The character is shown correctly on LEMAIRE's plate in the Fl. d. Serres Sér. 1. 4: pl. 388 (as D. cristata). The writer has examined the following specimens from outside Guiana:

Mexico: Guerrero: Montes de Oca distr., Hinton 10582 (U), 10616 (U).

OAXaCA: Tuxtepec distr., Martinez-Calderón 50 (GH).

Tabasco: Retiro Tenosique, Matuda 3440 (K).

Chiapas: Cacaluta, Escuintla, Matuda 16572 (BR).

Britrsh Honduras: El Cayo distr., Lundell 6549 (GH, S); Middlesex distr., Gentle 3016 (GH, K); Roaring Ck., Lundell 441 (A); Toledo, Peck 513 (GH, K, U); Punta Gorda, Schipp 1046 (A, BM, G, GH, K, MO, S, UC).

Guatemala: 'Cubilquitz, dept. Alta Verapaz, von Tuerckheim 7643 (GH), II 114 (BR, GH); Santa Rosa, dept. Santa Rosa, Heyde \& Lux 4034 (K); near Mazatenango, Bernoulli \& Cario 2014 (GOET).

EL Salvador: near Arachapa, dept. Sonsonate, Tucker 1345 (UC); Choussy. 48 (GH).

HondurAs: R. Lindo, dept. Cortes, Edwards 670 (A); Pito Solo, dept. Comayagua, Edwards 396 (A, K, UC).

Costa Rica: R. Frio, Holm \& Iltis 703 (BM, G, K, U); vic. of Alajuelita, Tonduz 8902 (BR, U); Santa Clara, J. D. Smith 6718 (GH, K); R. Maria Aguilar, Biolley 6958 (BR); Pittier 2550 (BR); Endres 184 (W).

Panama: vic. of Chiriquí Lagoon, prov. Bocas del Toro, von Wedel 1219 (MO, GH), 1272 (MO, GH), 1281 (MO, GH), 1388 (MO), 1555 (MO, GH), 1890 (MO), 2006 (MO, GH), 2052 (MO, GH), 2497 (MO); Boqueté, prov. Chiriquí, Davidson 828 (GH, MO); prov. Cocle, Allen 3557 (MO, BM, G); between Las Margaritas and El Valle, Woodson, Allen \& Seibert 1765 (MO, A); along R. Sirrí,prov. Golón, Pittier 4017 (BM); Fató, Nombre de Dios, prov. Colón, Pittier 3843 (GH); Chagres, Fendler 301 (K); Barro Colorado Island, C. Z., Bangham 415 (A), 460 (A); ibid., Salvoza 843 (A); ibid., Woodworth \& Vestal 371 (A); Manzanillo Island, C. Z., Hayes 23 (GH); San José Island, Gulf of Panama, Harlow 53 (GH); ibid., Johnston 62 (GH, MO).

Colombia: Magdalena: Santa Marta, Purdie 177 (GOET; $K$, type of $D$. parvifolia); ibid., Don Amo, H. H. Smith 1402 (NY, BM, BR, F, G, GH, K, L, P, PH, S, U, UC, US); ibid., Minca, Schlim 958 (G); San Pablo, Holton 609 (K, PH).

Norte de Santander: near La Mesita, Goudot 2 (P).

Santander: vic. of Puerto Berrio, Haught 1846 (US, BM, GH).

Cardas: west of Armenia, Cauca Valley, Pennell, Killip \& Hazen 8620 (GH, PH); between Coper and Mazo, Goudot, anno 1844 (P):

Tolmma: Ibaque, Goudot 1 (P).

Cauca: between La Sequia and Popayan, Humboldt \& Bonpland 2028 (P, type of $D$. spectabilis).

Putumayo: Umbria; Klug 1836 (BM).

ECUAdor: prov. Guayas, north of Pedro Carbo, Haught 3062 (UC).

Peru: Loreto: R. Putumayo, at mouth of R. Zubineta, Klug 2232 (GH, A, BM, G, K).

Madre de Dios: R. Acre, Ule 9774 (K).

Sin. loc., Pavon s.n. (G). 
Venezuela: Mérida: La Molina, Eggers 13525 (A).

Aragua: between Maracay and Choroni, Fendler 2033 (GH).

Monagas: R. Caripe, Caripe, Steyermark 62140 (F).

Amazonas: Chimborazo, R. San Antonio, Spruce s.n. (K).

Margarita IsLand: R. Asuncion, Johnston 278 (GH, G, F, K, NY, UC, W).

BraziL: GoIAs: near Goias City, Burchell 6455 (K).

Matto Grosso: Barro do Bugres (Santa Cruz), S. Moore 529 (BM, type of D. maculata).

ST. Thomas: Finlay 179 (P), 180 (P).

ST. Vincent: Caley 3 (P).

Toвago: Sandwith 1866 (K, NY, U).

Trinidad: near Sans Souci via Joco, Broadway 9053 (BM); Brazil via Guanapo, Broadway 5766 (BM, K); Diego Martin, Nurse 6857 (NY); near Iabaquite, Broadway, 15 Sept. 1918 (GH, NY); Siparia Quarry, Britton \& Broadway 2811 (G, GH, K, NY); Fendler 510 (BM, K), 513 (BM); Ryan 28 (C); anonym. (BM). Cult. (most important ones only): Kegel anno 1848, Hort. Gent (GOET); Hort. Knight, Sept. 1836, specimen depicted in Bot. Reg. (CGE); herb. Portenschlag, from Trinidad (W); herb. Jacquin, hort. Schönbrunn (W, type); Hort. Vienna (W).

D. calcarata Mart., Nov. Gen. 3: 58, t. 224. 1829; G. Don, Gen. Syst. 4: 653. 1838; D.C., Prod. 7: 543. 1839; Hanstein, Linnaea 26: 179, 207, 215, t. 2. f. 46.1853 ; in Martius, Fl. Bras. 8 (1): 404. 1864; Linnaea 34: 355. 1865; Fritsch in Engl., Bot. Jahrb. 37: 485. 1906.

Fig. 18, p. 392

Lectotype: BrazIL: Amazonas, near R. Japura, Martius 3014 (M). Heterotypic synonym: D. buscalionii Fritsch ex Buscalioni, Annali Bot. Roma 9: 113. 1911. Type: BraziL: Pará, near la Colonia di Annita Garibaldi, near Castanhal, Buscalioni 1765 (RO).

$D$. calcarata is about as variable as $D$. serrulata. It varies most in the indument and the shape of the sepals. From $D$. sernulata it differs by the shape of the ventral corolla lobe and the outside of the corolla as stated on p. 392.

Specimens from outside Guiana:

Brazil: ParA: near Castanhal, Buscalioni 1765 (RO, type of D. buscalionii); Belém, Silva $136(\mathbf{K})$.

AmazonAs: near R. Japura, Martius 3014 (M, lectotype), herb. 57 (M, paratype); near R. Madeira, Martius 3299 (M, paratype); Basin of R. Madeira, Municipality Humayta, near Tres Casas, Krukoff 6161 (F, K, MO, U); "Prov. R. Negro", Martius herb. 61 (M, paratype).

GuAPORE: near Tabajara, Upper Machado R. Region, Krukoff 1422 (G).

The specimen collected by Ule (3034 (HBG)) in Goias, cited by FrITsch (1906, p. 485 ), may belong to $D$. serrulata; it has no corolla. Both species occur in Brazil.

Episcia Mart., Nov. Gen. 3: 39. 1829.

This is one of the most critical genera of the family. Several species have been assigned to it which belong to various other genera and even to other tribes. It is allied to four other genera as stipulated in the numbers $6,9,12$, and 14 of the preceding paragraph. The subdivision of FRITSCH $(1894$, p. 166) cannot be maintained in the present author's opinion; especially the section Centrosolenia the type species of which, $E$. hirsuta, is more closely related to $E$. reptans than to any other species ascribed to it. Therefore the following subdivision is proposed: 


\section{Section 1. Episcia}

Mostly creeping terrestrial or epiphytic herbs with or without stolons. Leaf blades usually elliptic. Inflorescence usually sessile. Sepals lanceolate or rarely linear. Corolla trumpet-shaped or infundibuliform. Anthers all coherent or free; cells parallel or divergent at the base, confluent at the apex or not, dehiscent throughout or only partially. Placentae ovuliferous only on the inner or on both surfaces.

Subsection 1. Episcia (Cyrtodeira Hanst., Linnaea 26: 207. 1853, as genus).

Creeping stoloniferous mostly hairy plants. Stolons usually with one terminal plantlet. Leaves of a pair equal or subequal; blade often creamy along the midrib above and purple beneath, mostly bullate, elliptic, obtuse, rounded, or rarely acute at the apex. Sepals lanceolate, free or nearly so. Corolla trumpet-shaped. Anthers all coherent, orbicular or oblong; cells parallel, discrete, dehiscent throughout by a longitudinal split. Placentae ovuliferous on both surfaces.

Species: E. reptans Mart. (type species), E. cupreata (Hook.) Hanst., E. lilacina Hanst.

Subsection 2. Alsobia (Hanst.) Lwbg., stat. nov. (Alsobia Hanst., Linnaea 26: 207. 1853, as genus).

Creeping stoloniferous plants. Stolons with numerous plantlets in a series. Leaves of a pair equal or subequal; blade pale green, not bullate, elliptic, acute or obtuse at the apex. Sepals pale green, lanceolate, free. Corolla rather amply trumpet-shaped. Anthers nearly orbicular, all coherent; cells parallel, discrete, dehiscent throughout by a longitudinal split. Placentae ovuliferous only on the inner or on both surfaces.

Species: E. punctata (Lindl.) Hanst. (type species), E. dianthiflora Moore et Wilson.

Subsection 3. Physodeira (Hanst.) Lwbg., stat. nov. (Physodeira Hanst., Linnaea 26: 207. 1853, as genus).

Creeping without stolons. Leaves of a pair equal or subequal; blade bullate or not, elliptic, obtuse, rounded, or acute at the apex. Sepals lanceolate, free. Corolla, at least partially mauve, obliquely infundibuliform, above the base suddenly and strongly widened, especially ventrally, decurved. Anthers all coherent; cells parallel or slightly divergent at the base, discrete or obscurely connate at the apex, dehiscent throughout by a longitudinal split. Placentae ovuliferous on the inner surface only.

Species: $E$. bicolor Hook. (type species), E. silvatica Cuatr.

Subsection 4. Trematanthera Lwbg., subsect. nov.

Herbae stolonibus plantam parvam singulam gerentibus repentes. Folia cuiusque jugi aequalia vel subaequalia laminis ellipticis bullatis apice acutis. Sepala lanceolata paene libera. Corolla ample tubularis 
medio paulum curvata. Antherae reniformes omnes inter se cohaerentes loculis basi divergentibus apice connatis parte basali solum dehiscentibus. Placentae utraque facie ovuliferae.

Species: $E$. sphalera Lwbg.(type species), E. fimbriata Fritsch?.

E. sphalera Lwbg., sp. nov.

Fig. 27, p. 414

Herba terrestris stolonibus repens. Folia petiolata laminis ellipticis vel oblongo-ovatis utraque facie hirsutis. Flores axillares solitarii longe pedicellati. Sepala viridia foliacea lanceolata apice acuta. Corolla oblique infundibularis basi calcarata limbo patente. Stamina quattuor didynama filamentis basi omnibus connatis et basi corollae adnatis. Antherae oblongo-reniformes omnes inter se cohaerentes loculis basi divergentibus apice confluentibus et parte basali solum dehiscentibus. Ovarium superum hirsutum ovoideum. Stylus inclusus pilis glanduliferis vestitus. Disci glandula dorsalis solitaria. Placentae utraque facie ovuliferae. Capsula globosa bivalvis. Semina oblique striata.

Type: Suriname: Kappler 2044 (P, holotype, photograph: U, isotypes: GOET, RO, W, 2 sheets; distrib. as Tussacia villosa).

Complete diagnose see p. 413.

Paratype: French Guiana: Marowijne R., vic. of Codebert, Wachenheim 209 (P).

Etymology: $\sigma \varphi \alpha \lambda \varepsilon g o ́ \varsigma$, fallacious, as it resembles fallaciously $E$. cupreata from which it differs chiefly by its anthers and style.

Subsection 5. Centrosolenia (Benth.) Lwbg.; stat. nov. (Centrosolenia Benth., Lond. Journ. Bot. 5: 362. 1846, as genus; not of Morton, Field Mus. Publ. Bot. 18: 1158. 1938, etc.)

Creeping with or without stolons. Leaves of a pair equal or unequal; blade entirely green or above with a pale green area along the midrib and purple beneath, often bullate, elliptic or oblong-lanceolate. Inflorescence short-pedunculate or sessile. Sepals lanceolate or linear, acuminate. Corolla trumpet-shaped. Anthers coherent or not; cells parallel or divergent at the base, confluent at the apex or not, dehiscent throughout by a longitudinal split. Placentae ovuliferous on the inner surface only.

Species: $E$. hirsuta (Benth.) Hanst. (type species), $E$. peltata Morton, E. porphyrotricha Lwbg.

E. hirsuta (Benth.) Hanst., Linnaea 34: 350. 1865; Morton, Bull. Torr. Bot. Cl. 75: 566. 1948. Fig. 24, p. 406 1846.

Basionym: Centrosolenia hirsuta Benth., Lond. Journ. Bot. 5: 362.

Type: British Guiana: banks of Paramu R., Schomburgk s.n. (K, photographs: U, US).

Heterotypic synonym: Episcia cordata Gleason, Bull. Torr, Bot. Cl. 58: 466. 1931. Type: Venezuela: Amazonas, slopes of Mt. Duida, waterfall at Aguita, near Orinoco R., Tate 878 (NY, holotype, photograph: $\mathrm{U}$, isotypes: $\mathrm{K}, \mathrm{US})$.

In all characters both types fall within the variation of the species. Especially the leaves of this species are very variable in shape, size, and colour-pattern. 
Extra-Guianan specimens are:

Venezurla: Amazonas: Mt. Duida, Tate 878 (NY, K, US, type of E. cordata); ibid., Steyermark 57996 (F).

E. porphyrotricha Lwbg., sp. nov.

Fig. 26, p. 411

Herba terrestris. Folia cuiusque jugi inaequalia(?) petiolata laminis oblongo-lanceolatis apice acuminatis basi cuneatis vel in petiolum decurrentibus. Flores axillares solitarii vel bini. Sepala libera lineares. Corolla tubularis basi calcarata tubo cylindrico limbo patente. Stamina quattuor didynama inclusa filamentis basi omnibus connatis basi corollae adnatis. Antherae orbiculares omnes inter se cohaerentes loculis basi divergentibus discretis totam per longitudinem dehiscentibus. Ovarium superum hirsutum ovoideum. Stylus tubum corollae subaequans. Stigma bilobum. Disci glandula dorsalis singula. Placentae duae parietales bilamellatae facie interiore solum ovuliferae.

Type: British Guiana: Pakaraima Mts., Wenamu R., Davenport 7 (K, photographs: U, WAG).

Complete diagnose see p. 411.

Paratype: BRItrsh Guiana: Schomburgk 37 (K).

This species differs from the others of this section by the shape of the leaves and the sepals. By those characters it resembles some species of the section Paradrymonia from which it differs by its corolla with its narrow cylindric tube.

Section 2. Skiophila (Hanst.) Benth. et Hook. (Skiophila Hanst., Linnaea 26: 207. 1853, as genus).

Terrestrial mostly glabrous or pubescent herbs. Stems usually erect or ascending, sappy. Leaves of a pair equal or subequal; blade not bullate, acuminate at the apex. Inflorescence usually pedunculate and with some small green bracts. Pedicels slender. Sepals ovate, oblong, or lanceolate, free or connate at the base, acuminate at the apex. Corolla trumpet-shaped or infundibuliform. Anthers orbicular or oblong, coherent in pairs or free; cells parallel, hardly or not confluent at the apex, dehiscent throughout by a longitudinal split. Disk annular (teste Martius) or of one dorsal gland. Placentae ovuliferous on the inner surface only.

Species: E. melittifolia (L.) Mart. (type species), E. decumbens Mart., $E$. inclinata Brandegee, E. mimuloides Benth.

Section 3. Paradrymonia (Hanst.) Lwbg., stat. nov. (Paradrymonia Hanst., Linnaea 26: 207. 1853, as genus; taxonomic synonyms: Trichodrymonia Oerst., Centralamer. Gesn. 38. 1858; Centrosolenia Benth., exclusive of type species; Morton, Field Mus. Publ. Bot. 18: 1158. 1938; Ann. Mo. Bot. Gard. 29: 41. 1942; Journ. Wash. Acad. Sci. 35: 126. 1945).

Terrestrial or epiphytic herbs. Stems creeping or ascending, with many adventitious roots. Leaves of a pair equal to strongly unequal, usually, at least the longer one, surpassing the stem, mostly large; blade lanceolate, acuminate at the apex, cuneate at the base or often long-decurrent into the petiole. Inflorescence sessile, many-flowered. 
Sepals linear, free, long-acuminate. Corolla infundibuliform or trumpet-shaped. Anthers oblong, all coherent or coherent in pairs, often with bristles; cells parallel or divergent at the base, discrete, dehiscent throughout or only partially. Disk glands 1 or 2. Placentae ovuliferous on the inner surface only.

Species: E. glabra (Hook.) Hanst. (type species), E. buchtienii Mansf., $E$. ciliosa (Mart.) Hanst., E. congesta (Oerst.) Hanst., $E$. erythropus Hook. f., $E$. hansteiniana Mansf., $E$. longipetiolata Donn. Sm., Centrosolenia conferta Morton, $C$. decurrens Morton, C. lineata Morton, Alloplectus dariensis Seem.

For the species placed in this section that have been described in other genera no new combinations are made because at present it is not possible to investigate more in detail the boundaries between the species and their synonomy.

Section 4. Pagothyra Lwbg., sect. nov.

Herbae suffrutescentes repentes vel scandentes radicibus adhaesivis. Folia cuiusque jugi aequalia vel subaequalia longe petiolata laminis oblongo-ellipticis. Inflorescentia racemosa floribus crebris bracteis magnis sepala superantibus. Sepala lineari-lanceolata libera magna. Corolla infundibuliformis limbi lobis quattuor patentibus, quinto tubum occludente (teste Hooker). Antherae omnes inter se cohaerentes loculis parallelibus fissura longitudinali totam per longitudinem dehiscentibus. Disci glandula dorsalis singula. Placentae utraque facie ovula creberrima gerentes.

Species: E. maculata Hook. f.

Etymology: $\pi \alpha ́ \gamma \eta$, trap and $\vartheta \dot{v} \varrho a$, door, because the fifth corolla lobe closes the tube like a trap-door as figured by Hooker. From the other sections of Episcia it differs mainly by the habit, inflorescence, and large bracts.

Section 5. Trichosperma Lwbg., sect. nov.

Herbae suffrutescentes repentes. Folia cuiusque jugi valde inaequalia laminis oblongo-obovatis. Inflorescentia sessilis bracteis nonnullis parvis. Flores parvi. Sepala libera oblongo-lanceolata apice acuta. Corolla infundibuliformis. Antherae reniformes omnes inter se vel per paria cohaerentes loculis apice confluentibus totam per longitudinem dehiscentibus. Disci glandula dorsalis singula. Placentae facie interiore solum ovuliferae. Semina parce pilosa.

Species: $E$. bryogeton Lwbg.

This section differs chiefly from the other sections by the habit, the corolla, and the seeds. Its closest relative is section Episcia.

E. bryogeton Lwbg., sp. nov.

Fig. 22, p. 400 .

Herba suffrutescens terrestris vel epiphytica. Folia petiolata laminis oblongo-obovatis facie superiore tomentosis. Flores axillares fasciculati. Sepala viridia libera foliacea oblongo-lanceolata apice acuta. Corolla infundibuliformis basi breviter calcarata extus villosa limbo patente. Stamina quattuor didynama inclusa filamentis inter se liberis basi 
corollae adnatis. Antherae reniformes omnes inter se vel per paria cohaerentes loculis apice confluentibus totam per longitudinem dehiscentibus. Ovarium superum pilis longis molliter hirsutum globosum. Stylus glaber inclusus. Stigma stomatomorphum. Disci glandula dorsalis singula. Placentae facie interiore solum ovuliferae. Capsula globosa bivalvis. Semina parcissime pilosa oblique striata.

Type: British Guiana: Kurupung R., near Makreba Falls, Upper Mazaruni distr., Pinkus 12 (NY, holotype, photographs: U, WAG, isotype: US).

Complete diagnose on p. 400.

Paratype: British Guiana: Membaru Trail, Kurupung Mts., Altson 371 (K, 2 sheets).

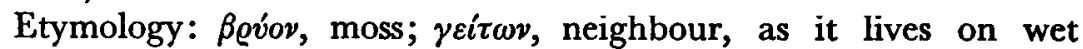
mossy rocks.

Its habit and leaves resemble exactly that of Cremosperma cotejense Morton of which it differs strikingly by its inflorescence and flowers.

Section 6. Salpinganthus Lwbg., sect. nov.

Herbae terrestres repentes. Caules saepe breves radicibus adventivis plerumque creberrimis repentes. Folia cuiusque jugi subaequalia longe petiolata caules longitudine vulgo superantes laminis oblongoellipticis vel oblongo-lanceolatis. Inflorescentia breviter pedunculata floribus crebris. Sepala oblongo-lanceolata apice acuta, quattuor pro parte tertio connata, quintum dorsale vulgo liberum. Corolla tubularis. Antherae reniformes per paria cohaerentes loculis basi divergentibus apice confluentibus fissura longitudinali totam per longitudinem dehiscentibus. Disci glandula dorsalis singula. Placentae facie interiore solum ovuliferae.

Species: $E$. densa C. H. Wright.

Etymology: $\sigma a ́ \lambda \pi \iota \gamma \xi$, trumpet, as the corolla is approximately trumpet-shaped.

This section is approximately intermediate between sect. $E$. subsect. Centrosolenia and sect. Skiophila. From the first it differs mainly by its habit and sepals, from the second by its sepals and anthers.

Section 7. Tarachanthus Lwbg., sect. nov.

Herbae repentes vel ascendentes. Folia cuiusque jugi subaequalia laminis ellipticis. Inflorescentia brevissime pedunculata bracteis nonnullis sepalis similibus. Sepala libera lanceolata apice acuminata. Corolla tubularis limbo magno. Antherae orbiculares omnes inter se cohaerentes loculis parallelibus discretis fissura longitudinali totam per longitudinem dehiscentibus. Disci glandula dorsalis singula. Placentae facie interiore solum ovuliferae.

Species: $E$. urticifolia Lwbg.

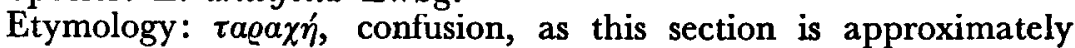
intermediate between sect. $E$. subsect. Episcia and sect. Skiophila. It is allied to sect. Episcia by its bullate leafblades and its anthers which are all coherent. Through its inflorescence, sepals, and placentation it resembles sect. Skiophila. 
E. urticifolia Lwbg., sp. nov.

Caulis radicibus fibrosis sparsis apice villoso-hirsutus glabrescens. Lamina apice acuminata basi oblique cuneata vel fere rotundata margine crenato-serrata. Inflorescentia axillaris umbellata. Sepala glabra vel facie exteriore solum pilosa. Corolla in calyce obliqua basi breviter calcarata extus pilosa. Stamina quattuor didynama inclusa filamentis basi omnibus connatis et basi corollae adnatis. Ovarium superum molliter hirsutum. Stylus glaber stamina longitudine subaequans. Stigma bilobum.

Type: Colombia: Chocó, between La Oveja and Quibdó, Archer
1698 (US).

Herb, 10-15 cm high. Stems creeping or ascending. Leaves of a pair subequal, petiolate; petiole 5-25 mm long; blade dark green above, pale green beneath, bullate, elliptic, about $2 \times$ as long as wide, $42-72 \times 22-35 \mathrm{~mm}$, papyraceous when dry, glabrous above, pilose beneath, especially on the midrib and veins. Inflorescence several-flowered. Peduncle very short with some sepal-like bracts which are approximately of the same size as the sepals. Pedicles glabrous, 6-14 mm long. Sepals free, lanceolate, 3-4.5 $\times$ as long as wide, $12-18 \times 3-5 \mathrm{~mm}$, acuminate at the apex, with some teeth, with scattered hairs along the margin, four erect, subequal, the fifth (dorsal) curved around the spur. Corolla salmon red (teste Archer) approximately trumpet-shaped, $1.5-2 \times$ as long as the calyx, 25-33 mm long, at the base $2.5-3 \mathrm{~mm}$, at the throat $4-5 \mathrm{~mm}$ wide, not contracted above the spur, slightly so in the throat, pilose outside, inside with glandular hairs in the throat; tube nearly cylindric, 15-20 mm long; limb spreading, proportionally wide, $22-26 \mathrm{~mm}$ wide; lobes unequal, rounded, entire, the ventral one $12 \mathrm{~mm}$ in diam., the others smaller. Filaments glabrous, not contorted; anthers orbicular, $1.7 \mathrm{~mm}$ in diam., all coherent in a square; cells parallel, discrete, dehiscent throughout by a longitudinal split. Staminode one, very small, about $0.5 \mathrm{~mm}$ long, glabrous, with an obscure sterile anther. Ovary ovoid, $3 \times 1.5 \mathrm{~mm}$. Disk gland 1, dorsal, entire, glabrous. Placentae ovuliferous on the inner surface only. Fruit and seeds not seen.

Paratype: Colombia: Antioquia, Medellin, Boxall s.n. (W, herb. 42551).

The key presented in the following is wholly based on characters visible without dissection of flowers. This appeared to the present author to be the most practical solution as herbarium specimens of Gesneriaceae have usually only very few flowers which are not rarely mounted in such a way that analysis of their interior characters would result in total destruction. It is a matter of course that with such an almost entirely artificial key individual species rather than sections are keyed out. More or less the same is true for the key in the last chapter of the present study (p. 355).

Key to the species (except section Paradrymonia):

1. Inflorescence racemose, with large green, purple- or redveined bracts; stems with a unilateral line of adhesive roots (like Hedera). British Guiana . . . E. maculata

Inflorescence if several-flowered umbellate, pedunculate or sessile, without or with small bracts . . . . . . . .

2. Flowers small; corolla about $12 \mathrm{~mm}$ long; leaves of a pair strongly unequal, all petiolate, the smaller one often deciduous; blade tomentose above. BRITISH GuIANA 
Flowers larger, at least $15 \mathrm{~mm}$ long; leaves glabrous or

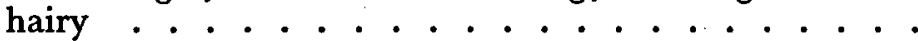

3. Leaves peltate, bullate; plant hirsute. Colombia E. peltata

Leaves not peltate, bullate or not; plant hirsute or not

4. Sepals linear, often purple, free; leaves usually longer than the stems; blade oblong-lanceolate, acuminate, cuneate or decurrent into the petiole, not bullate . . . . .

Sepals ovate, oblong, or lanceolate, free or connate; leafblades mostly glabrous, or hirsute and bullate if oblonglanceolate

5. Corolla crimson, about 5 cm long, trumpet-shaped, tube narrow, hardly widened towards the throat; plant hirsute; inflorescence few-flowered. British GuIANA . . . . .

E. porphyrotricha

Corolla usually creamy or white, about $2-4 \mathrm{~cm}$ long, infundibuliform or trumpet-shaped; inflorescence sessile, manyflowered; stems with many adventitious roots.

Sect. Paradrymonia

6. At least four of the sepals connate at the base for one-third of their length, acute at the apex, sparsely pilose to glabrous; leaves mostly glabrous or nearly so. GUIANA. . .

E. densa

Sepals free or connate at the base, acuminate, if acute plant hirsute . . . . . . . . . . . . . .

7. Plant hirsute; leafblades usually bullate . . . . . .

Plant glabrous, pubescent, strigillose, or pilose; leafblades not bullate .............. 17

8. Corolla red . . . . . . . . . . . . . . . 9

Corolla white and/or mauve .......... . 11

9. Leafblades glabrous above; plants creeping or ascending, without stolons . . . . . . . E. urticifolia

Leafblades hirsute above; plant stoloniferous . . . . .

10. Corolla tube $30-35 \mathrm{~mm}$ long, nearly straight, slightly widened, pinkish in the throat, all lobes spreading. CoLombia, Guiana, Brazil . . . . . • E. reptans

Corolla tube up to $25 \mathrm{~mm}$ long, suddenly decurved in the middle and from there more widened, yellow in the throat, 2 dorsal lobes recurved. Colombia, Venezuela, GuiANA ............ E. cupreata

11. Corolla about $3 \times$ as long as the width of the limb, usually more than $5 \mathrm{~cm}$ long ............

Corolla about $1-1 \frac{1}{2} \times$ as long as the width of the limb, 2-4 cm long ..............

12. Leafblades bullate, usually obtuse at the apex; corolla $47-70$ mm long. Guayana Highlands . . . E. hirsuta

Leafblades not bullate, acuminate; corolla about $4-5 \mathrm{~cm}$ long. Mexico . . . . . . . . E. inclinata

13. Inflorescence shortly pedunculate; pedicels slender or not; flowers often more than five; corolla tube ventrally 
suddenly and strongly widened, 20-25 mm long; plants without stolons . . . . . . . . . . . . . . . . . .

Inflorescence sessile; pedicels slender; flowers 1-4; corolla tube usually gradually widened; plants stoloniferous . .

14. Leafblades not bullate, green beneath; corolla purple-spotted inside; flowers numerous. Colombia . . . E. bicolor

Leafblades bullate, purple beneath; corolla not spotted; flowers several. Colombia . . . . . . E. silvatica

15. Corolla throat narrow; lobes conspicuously wider than the tube; stamens included; anthers oblong; cells parallel, discrete, dehiscent throughout by a longitudinal split. Nicaragua, Costa Rica, Panama, Colombia E. lilacina

Corolla throat wide; lobes not or hardly wider than the throat; stamens when filaments not contorted sligtly exserted; anthers reniform; cells divergent at the base, confluent at the apex, partially dehiscent at the base

16. Corolla about $25 \mathrm{~mm}$ long; leaves entirely green. GuiAnA

Corolla 30-41 mm long; leaves with purple margin beneath. Peru, Brazil (Matto Grosso) . . E. fimbriata? 1)

17. (7). Sepals acute; plants stoloniferous; corolla limb fimbriate . . . . . . . . . . . . . . .

Sepals acuminate; plants without stolons; corolla limb not fimbriate . . . . . . . . . . . . . . .

18. Corolla limb spotted; leafblades acute. Mexico, GuateMalA . . . . . . . . . . . . . . . . E. punctata

Corolla limb not spotted; leafblades obtuse. Mexico . . .

. . . . . . . . . . . . . . E. dianthiflora

19. Corolla creamy or white, $40-60 \mathrm{~mm}$ long . . . . . . .

Corolla purple or crimson, 15-30 mm long. Lesser ANTILLES

20. Leafblade decurrent into the petiole. Guiana, Toвago E. melittifolia Leafblade not decurrent into the petiole. BrazIL.

............... E. decumbens

Species of doubtful identity:

E. longifolia (Poepp. et Endl.) Hanst., Linnaea 34: 350. $1865=$ Nautilocalyx sp.?.

E. luciani Lind. et Fourn., Illustr. Hortic. 23: 43, pl. 236. 1876.

E. ochroleuca (Poepp. et Endl.) Hanst. in Martius, Fl. Bras. 8 (1): 403. $1864=$ Besleria sp.?.

Centrosolenia aenea Lind. et André, Illustr. Hortic. 22: 168, pl. 222. 1875.

1) Type not seen; characters taken from specimens identified with Frrtsch's description (1906, p. 484). 
Excluded species except those transferred to Nautilocalyx by SpRague (1912):

E. aurea Brandegee, Univ. Calif. Publ. Bot. 6: 374. $1917=$ Besleria pansamalana Donn. Sm.

E. cuneata Gleason, Bull. Torr. Bot. Cl. 58: 467. 1931 = Tylosperma cuneatum (Gleason) Lwbg.

E. elongata Jackson, Ind. Kew. 1: 856. $1895=$ "Gesneria" elongata Ann. Fl. et Pom. 1838: 188.

E. hispida D.C., Prod. 7: 547. $1839=$ Diastema hispidum (D.C.) Fritsch.

E. hookeri Hanst., Linnaea 34: 350. $1865=$ Codonanthe crassifolia (Focke) Morton.

E. lanceolata Hanst., Linnaea 34: 346. $1865=$ Drymonia lanceolata (Hanst.) Morton.

E. pulchella (Donn ex Sims) Mart. ex G. Don, Gen. Syst: 4: 656. $1838=$ Chrysothemis pulchella (Donn ex Sims) Dcne.

E. purpusii (Brandegee) Brandegee, Univ. Calif. Publ. Bot. 6: 194. $1915=$ Drymonia sp. aff. lanceolata (Hanst.) Morton.

E. rosea Mart. et Galeotti, Bull. Acad. Brux. 9 (2): $39.1842=$ Tetranema mexicanum Benth. (Scrophulariaceae).

E.? subacaulis Griseb., Fl. Br. W.I. Isl. 462. 1861 ("1864") = Napeanthus subacaulis (Griseb.) Benth. et Hook. ex Kuntze.

E. truncicola Brandegee, Univ. Calif. Publ. Bot. 6: 64. $1914=$ Hypocyrta nummularia Hanst.

Kohleria Regel, Flora 31: 250. 1848.

K. hirsuta (H.B.K.) Regel, l.c.; Hanstein, Linnaea 34: 441. 1865; Urban, Symb. Ant. 2 : 369. 1901; H.E. Moore, Baileya 1 : 99, f. 50A. 1953; Morton in Fl. Trin. and Tob. 2 (5) : 313.1955.

Basionym: Gesneria hirsuta H.B.K., Nov. Gen. 2: 317, t. 189.1818 ("1817"); D.C., Prod. 7: 530. 1839.

Type: Venezuela: Sucre, Cumaná, Caripe, Humboldt \& Bonpland 330 (B, herb. Willdenow 11717, holotype, not seen, photograph: U, isotypes: $P, 2$ sheets!).

Homotypic synonyms: Isoloma hirsutum (H.B.K.) Regel, Bot. Zeit. 9: 893 (not 583). 1851 ; Grisebach, Fl. Br. W.I. Isl. 459. 1861 (“1864"). Brachyloma hirsutum (H.B.K.) Hanst., Linnaea 26: 203. 1853; op. cit. 29: 526, 569. 1858.

Heterotypic synonym: Brachyloma rhodomallon Hanst., Linnaea 29: 526, 570. 1858. Type: Venezuela: Mérida, Moritz 414 (BR, CGE, $\mathrm{L}, \mathrm{P}$, isotypes).

Homotypic synonym: Kohleria rhodomallos (Hanst.) Hanst., Linnaea 34: 441. 1865; Fritsch in Engl., Bot. Jahrb. 37: 420.1913.

After comparison of the types and the other specimens extant it turned out that none of the differential characters between $K$. hirsuta and $K$. rhodomallos which Hanstein (1858, p. 526, 529) listed can be used. All of them are gradually and independently variable. For instance the red venation of the leaves was used as an important 
character, but in one single specimen the leaves may be red-veined or entirely green beneath.

Extra-Guianan specimens are:

Colombia: Magdalena: east of Manaure, Haught 4345 (UC).

Norte de Santander: vic. of Ocaña, Schlim 93 (BR, CGE, G, P).

SANTANDER: between El Roble and Tona, Killip \& Smith 19412 (W).

Venezuela: TÁchira: between Las Delicias and Paraguita, Steyermark 57145 (F, US).

MÉrida: Laguna Grande, Páramo Mucuchíes, de Bellard 18 (US); Moritz $1127(\mathrm{~L}, \mathrm{P})$.

MÉRIDA?: near Tovar, Fendler 792 (P), 793 (BR, GOET, PH).

Trujillo: near Escuque, Pittier 13140 (F, G, PH).

Portuguesa?: Las Adjuntas, Eggers 13358 (C, L, US).

Cojedes: Los Chorros, Eggers 13078 p.p. (L).

D.F.: between La Guaira and Caracas, herb. Kuntze 1383 (K); ibid., Pittier 9864 (US); ibid., Rose \& Rose 21739 (US); vic. of Caracas, Bailey \& Bailey 731 (US); ibid., Moritz 414 (BR, CGE, L, P, isotypes of $K$. rhodomallos); herb. Buek (leg. Moritz?) s.n. (HBG); ibid., Plée s.n. (P); ibid., Steyermark 55037 (F, US); near Galipán, Funck \& Schlim 152 (P); ibid., Pittier 104 (US).

Aragua: El Castaño, Ll. Williams 10455 (US).

Mrranda: above Los Teques, Pittier 11251 (US); El Encanto, Allart 152 (US).

SuGRE: Caripe, Funck \& Schlim 242 (BR, P, U); ibid., Cumaná, Humboldt \& Bonpland 330 (P, 2 sheets, isotypes); Moritz 413 (BM, K).

Bolfvar: Quebrada O-paru-má, trib. of R. Pacairao, Steyermark 60582 (F, US). Sin. Loc.: Linden 42 (BR).

Trinidad: North Coast Road to Maracas Bay, herb. Trin. 14139 (US), 14729 (K); Maracas, Crueger, 10 Nov. 1845 (K, GOET); ibid., herb. Trin. 15061 (K); Mt. Tucuche, Britton, Hazen \& Mendelson 1304 (K, US).

Lembocarpus Lwbg., gen. nov.

Herba acaulis humilis terrestris vel epiphytica $5-20 \mathrm{~cm}$ alta tubero parvo petiolos pedunculosque gerente. Folia plerumque solitaria petiolata; lamina cordato-orbicularis apice acuta vel obtusa basi cordata margine irregulariter sinuato-dentata subpalmativenia. Inflorescentia axillaris solitaria. Flores in dichasium asymmetricum interdum ad florem singulum redactum dispositi. Pedunculus gracilis folia longitudine fere aequans bracteis parvis suffultus. Sepala quinque viridia libera aequalia lineari-lanceolata trinervia. Corolla tota pallide caerulea vel corollae tubus albus et limbus lilacinus vel corolla alba et limbo facie interiore purpureo-maculato, ample tubulosa vel campanulata in calyce obliqua calycem longitudine $2-4 \times$ superans ecalcarata basi latere dorsali gibbosa supra gibberum et fauce non contracta vix ventricosa; limbus patens paulum obliquus lobis quinque subaequalibus late rotundatis. Stamina quattuor didynama inclusa; filamenta non contorta inter se libera basi corollae inserta; antherae oblongae per paria cohaerentes loculis parallelibus discretis fissura longitudinali dehiscentibus. Ovarium superum; stylus inclusus deciduus; stigma bilobum facie superiore pilis glanduliferis pubescens. Discus annularis angustus integer. Placentae duae parietales bilamellatae laminis facie exteriore solum ovuliferis. Fructus capsularis bivalvis loculicidus. Semina immatura nitentia oblique ellipsoidea longitudinaliter vel oblique striata. 


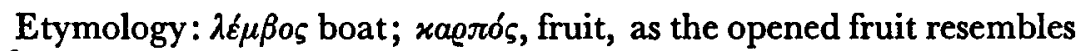
a boat.

Only one species in the mountains of Suriname.

This peculiar plant is distantly related to Gloxinia by the shape of the corolla and the disk, but differs widely from it by its entirely superior ovary, the inflorescence, and the absence of stems (p. 297). Furthermore it is related to Rhoogeton as pointed out on p. 322.

L. amoenus Lwbg., sp. nov.

Fig. 28, p. 419

Tuber globosus radicibus fibrosis vestitus. Folia laminis paulum obliquis. Pedunculus et pedicelli villosi vel pilosi. Bracteae linearilanceolatae. Sepala acuminata integra vel obscure sinuato-dentata. Corolla extus pilosa vel villosa intus glabra limbo subactinomorpho. Ovarium puberulum ovoideum. Stylus glaber.

Type: Suriname: Nassau Mts., Maguire 40806 (U).

Complete diagnose see p. 418.

Paratypes: Suriname: Nassau Mts., Lanjouw \& Lindeman 2466 (U), 2593 (U), 2833 (U); ibid., Maguire 40788 (U); Wilhelmina Mts., B.W. 7077 (U); Emmaketen, B.W. 5807 (U).

The following specimens belong to Lembocarpus and perhaps to $L$. amoenus, but they differ from the others by their white corolla with purple dots (according to field notes):

Suriname: Tafelberg, Maguire 24335 (NY), 24748 (NY).

Nautilocalyx Linden, Cat.'1851: 12 (fide Hanstein, Linnaea 26: 181, 207. 1853).

As defined by SPRAGUE. (1912) this is a natural and useful unit, although it is very difficult to state by virtue of what characters it forms an entity. It is closely related to Alloplectus and Episcia (more distantly to Drymonia and Sinningia), being approximately intermediate between Alloplectus section Heintzia and the sections Skiophila and Paradrymonia of Episcia as stipulated in the numbers 4, 6, 8, and 13 of the preceding paragraph.

N. pictus (Hook.) Sprague, Kew Bull. 1912: 88.

Basionym: Centrosolenia picta Hook., Bot. Mag. 77: t. 4611. 1851. Type: Cult. in Kew Gardens from a collection of Spruce in Brazil (K, photographs: U, US). Homotypic synonym: Episcia picta (Hook.) Hanst. in Martius, Fl. Bras. 8 (1): 403. 1864; Linnaea 34: 349.1865.

Misapplied names: Collandra picta (Hook.) Lem., Jardin fleuriste 2: pl. 214. 1852, not Hanst.

Columnea(?) picta (Hook.) Lem. ex Hanst. in Martius, Fl. Bras. 8 (1): 422. 1864, not Karsten.

Heterotypic synonym: Nautilocalyx lacteus Sandwith, Kew Bull. 1931: 489. Type:- British Guina: Essequibo -R., Moraballi Gk., near Bartica, Sandwith 3 (K, photographs: U, US).

$\mathcal{N}$. lacteus cannot be maintained as a distinct species on the following grounds: 
Leaves from sparsely strigose to glabrous above. Corolla not always entirely white, in duplicate sheets of some paratypes of $\mathcal{N}$. lacteus inside purple spotted as figured by Hooker (Bot. Mag. 4611), never purple lined as erroneously stated by Hanstein (1864, p. 403). Filaments glabrous or with some hairs near the apex, in the type of $\mathcal{N}$. lacteus connective pilose. Disk gland mostly one, dorsal, rarely also a ventral gland.

The only extra-Guianan report is that of Spruce's collection which is also the origin of the type.

N. villosus (Kunth et Bouché) Sprague, Kew Bull. 1912: 89.

Basionym: Drymonia villosa Kunth et- Bouché, Ind. Sem. Hort. Berol. 1847: 12 (fide Walpers, Ann. 1: 474. 1848-1849); Hooker, Bot. Mag. 81 : t. 4866.1855.

Type not seen, destroyed in Berlin?

Homotypic synonym: Episcia villosa (Kunth et Bouché) Hanst., Linnaea 34: 348. 1865.

In the present study HookeR's plate (Bot. Mag. 4866) and WALPERs' description (1848-'49, p. 474) were taken as base for this species, as neither an isotype nor the original description were at hand. It was reported by Hooker from Suriname, but the only Guianan specimen examined by the present author (collected by Benoist) is of doubtful identity. The colour of the flowers is not noted, the limb of the corolla is damaged, and the sepals are proportionally wider. Venezuela: D.F.: Caruao, Pittier 11930 (US).

French Guiana: St. Jean, Benoist 820 (P)?

Cult.: Hort. Kew. s.n. (K, specimen depicted in Bot. Mag.); Hort Kew., July 1860 (K); Hort. Berol., anno 1855 (W); Hort. Petrop. (LE).

Rechsteineria Regel, Flora 31 : 247. 1848.

This genus is represented in Guiana by the section Corytholoma Benth. with two species, $R$. incarnata and $R$. schomburgkiana.

R. incarnata (Aubl.) Lwbg., comb. nov.

Figs. 29, p. $426 ; 30$ I, p: 428

Basionym: Besleria incarnata Aubl., Hist. Pl. Guian. 2: 635, and 4: t. 256. 1775; G. Don, Gen. Syst. 4: 652. 1838; D.C., Prod. 7: 539. 1839; Hanstein, Linnaea 34: 421. 1865.

Type: French Guiana: basin of Galibi Ck., Aublet s.n. (BM, flowers lost).

Heterotypic synonyms: Gesneria chelonioides H.B.K., Nov. Gen. 2: 315. 1818 ("1817"); G. Don, l.c., p. 647; D.C., l.c., p. 527; Hanstein, Linnaea 34: 262. 1865 (as Gesnera). Type: Colombia: Cauca, near the glens of the R. Guilguase and R. Smitha, between Popayan and Amalguer, Humboldt \& Bonpland s.n. (P-BO, photographs: F, U, US).

Homotypic synonyms : Corytholoma chelonioides (H.B.K.) Regel, Gartenflora 7: 374. 1858.

Rechsteineria chelonioides (H.B.K.) Kuntze, Rev. 2: 474. 1891. 
Gesneria rutila Lindl., Bot. Reg. 14: t. 1158. 1828; Martius, Nov. Gen. 3: 34.1829 (as Gesnera); G. Don, 1.c., p. 648; D.C., l.c., p. 529; Hanstein in Martius, Fl. Bras. 8 (1): 372. 1864; Linnaea 34: 270. 1865 (as Gesnera). Type: Cult. from South America, G. de Vandes, Sept. 1827 (CGE, holotype, photographs: U, WAG).

Homotypic synonyms: Corytholoma rutilum (Lindl.) Dcne., Rev. Hortic. Sér: 3. 2: 467. 1848.

Rechsteineria rutila (Lindl.) Kuntze, l.c.

Gesneria lindeniana Brongn., Rev. Hortic. Sér. 3. 1: 363. 1847. Type: Venezuela: Mérida, Linden 1408 (G, holotype, isotypes: BM, F, G, $\mathrm{K}, \mathrm{P}, \mathrm{W}, 2$ sheets).

Gesneria stachydifolia Benth., Pl. Hartweg. 230. 1846; Hanstein, Linnaea 34: 263. 1865 (as Gesnera stachyfolia). Type: Colombia: Cauca, vic. of Popayan, opposite the junction of $R$. Sucio with $R$. de las Botas, Hartweg 1259 (K, holotype, photographs: U, WAG).

Homotypic synonym: Rechsteineria stachydifolia (Benth.) Kuntze, l.c.

Inflorescence terminal, thyrsoid; bracts leafy, deminishing in size towards the apex or not; when not, the flowers stand actually in the axils of common leaves. The degree of reduction of the bracts and the number of flowers in each inflorescence may vary as figured in Figure 29. The diversity of several individuals of one collection is evident in the various sheets of H. H. Smith 1398 and Linden 1408. The Colombian and some Venezuelan specimens have slightly shorter and more or less appressed hairs on the stems and the pedicels than the Guianan ones, but some Venezuelan are intermediate in this character.

Extra-Guianan specimens are:

Colombia: Magdalena?: Santa Marta, H. H. Smith 1398 (BM, BR, G, K, L, P, S, U).

Norte DE Santander: Ocaña, Cucutilla, Kalbreyer 1048 (K); ibid.?, Kalbreyer s.n. (BM).

T'olima: between Melgar and Pandi, Goudot 6 (P); vic. of Ibagué, Linden $981(\mathrm{~W})$.

CAucA: between Popayan and Amalguer, Humboldt \& Bonpland s.n. (P-BO, type of $\boldsymbol{R}$. chelonioides); ibid.?, Bonpland $2032(\mathrm{P})$; vic. of Popayan, Hartweg 1259 ( $\mathrm{K}$, holotype of $R$. stachydifolia), Sept. 1844 (CGE); ibid., Langlassé 81 (P, G, U); Aganche, Cauca Valley, Pennell \& Killip 8329 (PH).

Venezuela: Mérida: Linden 1408 (BM, F, G, K, P, W, type of Gesneria lindeniana); Funck \& Schlim 1517 (BM, G, P, W).

MÉrIDA?: near Tovar, Fendler 795 (G, P, PH).

Cojedes: Los Chorros, Eggers 13078 p.p. (A).

Carabobo: Montalbán, Funck \& Schlim 656 (BM, CGE, G, P, U, W).

D.F.: Lower Cotiza R., Pittier 7894 (PH).

Miranda: between Petare and Guarenas, Ll. Williams 11146 (F).

Amazonas: near R. Negro, vic. of San Carlos, Spruce 3024 (AWH, BM, BR, CGE, G, K, P, W), $3149(\mathrm{~K}, \mathrm{P})$.

Sin. loc., Grosourdy, anno 1862 (W).

Cult.: C. de Vandes, from South America, Sept. 1827 (CGE, holotype of $R$. rutila); several specimens in: $B R, G, K, L, M, P, W$.

Rhoogeton Lwbg., gen. nov.

Herba acaulis terrestris tubero parvo radicibus fibrosis vestito vel sine tubero (?). Folia radicularia petiolata; lamina rotundata, ovata 
vel oblongo-ovata penninervia. Inflorescentia axillaris umbellata, subcymosa vel thyrsoidea longe pedunculata 1-6-flora bracteis lanceolatis suffulta. Sepala quinque viridia libera oblonga vel lanceolata apice acuta. Corolla tubularis in calyce obliqua basi breviter calcarata limbo patente. Stamina quattuor didynama inclusa filamentis inter se liberis prope basin corollae insertis. Antherae vulgo cohaerentes loculis parallelibus discretis fissura longitudinali totam per longitudinem dehiscentibus. Staminodium perparvum anantherum singulum. Ovarium superum. Stylus inclusus. Stigma bilobum utraque facie pilis glanduliferis pubescens. Disci glandula dorsalis singula magna emarginata glabra. Placentae duae bilamellatae facie exteriore solum ovuliferae.

Type species: R. cyclophyllus Lwbg.

Leafblades often proliferous at the margin with small bulb-shaped (?) rooting plants; midrib and veins prominent beneath. Sepals with 3 longitudinal veins. Corolla orange or red (?), nearly trumpet-shaped, slightly widened, somewhat contracted above the spur and in the throat, slightly ventricose, somewhat bent downwards, sparsely pubescent or strigillose outside, inside with glandular hairs in the throat; lobes 5 , more or less unequal, rounded. Filaments contorted or not; anthers orbicular, square, or oblong. Fruit and seeds not seen.

Two species in the mountains of British Guiana.

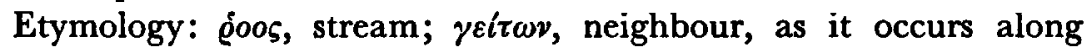
streams.

This genus is related to Episcia from which it differs as follows: Caulescent; peduncle much shorter than the leaves; placentae ovuliferous only on the inner or on both surfaces ...... Episcia Acaulescent; peduncle elongate, often longer than the leaves; placentae ovuliferous only on the outer surface ... . Rhoogeton

The rather narrow corolla resembles that of Episcia sect. $E$. subsect. $E$. The same is true for the stamens and the calyx. On the other hand Rhoogeton is allied to Lembocarpus by its tubers, venation of the calyx, inflorescence, absence of stems, and placentation. From this it keys out as follows:

Corolla campanulate, not spurred, not contracted in the throat, glabrous inside; disk annular ........ Lembocarpus Corolla nearly trumpet-shaped, spurred at the base, contracted in the throat, inside with glandular hairs in the throat; disk of one

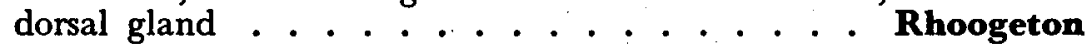

R. cyclophyllus Lwbg., sp. nov.

Fig. 31, p. 430

Herba tenuis tubero parvo radicibus fibrosis vestito. Petiolus tenuis. Lamina orbicularis apice rotundata basi cordata margine grosse crenato-serrata. Inflorescentia subcymosa vel thyrsoidea 1-6flora. Filamenta glabra non contorta. Antherae omnes inter se cohaerentes vel liberae.

Type: British Guiana: Mt. Ayanganna, Pakaraima Mts., Maguire, Bagshaw \& Maguire 40585 (NY, photographs: U, WAG). .

Complete diagnose see p. 429.

Paratype: British Guiana: Kaieteur, Jenman 896 (K, photograph: U). 
R. viviparus Lwbg., sp. nov.

Fig. 32, p. 431

Lamina ovata vel oblongo-ovata apice acuminata basi rotundata vel oblique cuneata margine biserrato-dentata. Inflorescentia umbellata vel subcymosa 1-6-flora. Filamenta glabra contorta. Antherae onmes inter se vel per paria cohaerentes vel liberae (?).

Type: British Gulana: Kaieteur, Jenman 880 (P, holotype, photographs: $\mathrm{U}, \mathrm{WAG}$, isotypes: $\mathrm{K}, \mathrm{U})$.

Complete diagnose see p. 431.

Paratype: BRITISH GUIANA: Kaieteur, Jenman $888(\mathrm{~K})$.

The species of Rhoogeton differ as stipulated on p. 429.

Tylosperma Lwbg., gen. nov.

Herba terrestris. Caulis brevissimus radicibus fibrosis obtectus. Folia radicularia rosulata petiolata vel subsessilia laminis oblanceolatis apice acuminatis basi breviter vel longe decurrentibus. Inflorescentia axillaris foliis brevior paniculata floribus parvis numerosis. Pedunculus pedicellique tenues et ebracteati. Sepala basi connata linearilanceolata longe acuminata. Corolla alba tubularis basi gibbosa limbo patente. Stamina quattuor inclusa. Filamenta inter se libera prope basin corollae inserta. Antherae reniformes per paria cohaerentes loculis basi divergentibus apice confluentibus fissura longitudinali totam per longitudinem dehiscentibus. Staminodium parvum anantherum. Ovarium superum. Stigma capitatum. Disci glandulae quinque, una dorsalis magna, ceterae parvae. Placentae utraque facie ovuliferae. Fructus capsularis valvis duabus vel quattuor(?) translucentibus, loculicidus et etiam septicidus(?). Semina nitentia subglobosa pustulata basi oblique mucronata.

Only one species: T. cuneatum (Gleason) Lwbg., comb. nov.

Basionym: Episcia cuneata Gleason, Bull. Torr. Bot. Cl. 58: 467. 1931.

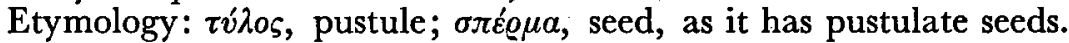

This genus has been separated from Episcia on the following grounds: Inflorescence paniculate, long-pedunculate, ebracteate. Disk glands five instead of one. Seeds globose, somewhat flattened, pustulate instead of striate.

The indument of $T$. cuneatum may vary, especially on the leaves which are subtomentose to glabrous.

Specimens from outside Guiana are:

Venezuela: Amazonas: Mt. Duida, Tate 879 (NY, holotype, photographs: U, WAG, isotypes: K, US); ibid., Steyermark 57992 (F, US); near R. Casiquiare, Spruce 3379 (AWH, BM, BR, CGE, G, GH, GOET, NY, P, U, W).

GHAPTER 3

\section{NOTES ON GEOGRAPHICAL DISTRIBUTION AND ECOLOGY}

This rather large family of which about 1800 species have been described occurs in America throughout the whole tropical region 
TABLE

Geography of genera of Gesneriaceae occurring in Guiana

\begin{tabular}{|c|c|c|}
\hline Genus & Area & $\begin{array}{l}\text { Centre of species } \\
\text { concentration }\end{array}$ \\
\hline Alloplectus & $\begin{array}{l}\text { West Indies, Mexico, Central and South America } \\
\text { south to Peru, Bolivia, and southern Brazil. }\end{array}$ & Colombia \\
\hline Besleria & $\begin{array}{l}\text { West Indies, Mexico, Central and South America } \\
\text { south to Peru, Bolivia, and southern Brazil. }\end{array}$ & Colombia \\
\hline Chrysothemis & $\begin{array}{l}\text { West Indies, Guatemala to Colombia, Venezuela, } \\
\text { British Guiana, Suriname, and Brazil (Amazonas). }\end{array}$ & $\begin{array}{l}\text { Guayana } \\
\text { Highlands? }\end{array}$ \\
\hline Codonanthe & $\begin{array}{l}\text { Trinidad, Tobago, Mexico, Central and South } \\
\text { America south to Peru and southern Brazil. }\end{array}$ & Colombia \\
\hline Columnea & $\begin{array}{l}\text { West Indies, Mexico, Central and South America } \\
\text { south to Peru, Bolivia, and southern Brazil. }\end{array}$ & Colombia \\
\hline Drymonia & $\begin{array}{l}\text { West Indies, Mexico, Central and South America } \\
\text { south to Peru, Bolivia, and southern Brazil. }\end{array}$ & Colombia? \\
\hline Episcia & $\begin{array}{l}\text { Lesser Antilles, Mexico, Central and South America, } \\
\text { south to Peru, Bolivia, and southern Brazil. }\end{array}$ & Colombia \\
\hline Gloxinia & $\begin{array}{l}\text { Mexico, Central and South America south to Peru, } \\
\text { Bolivia, and southern Brazil. }\end{array}$ & Colombia? \\
\hline Kohleria & $\begin{array}{l}\text { Trinidad, Mexico, Central and South America south } \\
\text { to Peru, Bolivia, and southern Brazil. }\end{array}$ & Colombia \\
\hline Lembocarpus & Suriname & 一 \\
\hline Napeanthus & $\begin{array}{l}\text { Trinidad, Tobago, Central and South America south } \\
\text { to Peru, Bolivia, and southern Brazil. }\end{array}$ & Colombia? \\
\hline Nautilocalyx & $\begin{array}{l}\text { Colombia, Peru, Venezuela, Guiana, and northern } \\
\text { Brazil. }\end{array}$ & Colombia \\
\hline Rechsteineria & $\begin{array}{l}\text { Mexico and South America from Colombia to } \\
\text { northern Argentina and Uruguay. }\end{array}$ & $?$ \\
\hline Rhoogeton & British Guiana & - \\
\hline Tylosperma & Guayana Highlands & - \\
\hline
\end{tabular}

and extends somewhat into the southern temperate region. In the North the family extends to approximately the $18^{\circ} \mathrm{N}$. Lat. in Mexico and to the Greater Antilles and in the South to Chile, northern Argentina, and Uruguay. All American genera except Klugia are restricted to the Western Hemisphere. On the continent representatives of the family occur up to considerable elevations in the mountains, 
Number in parentheses of species only known from that region.

\begin{tabular}{|c|c|c|c|c|c|c|}
\hline $\begin{array}{l}\text { Total number } \\
\text { of species }\end{array}$ & $\begin{array}{l}\text { No. of species } \\
\text { in Guiana }\end{array}$ & $\begin{array}{l}\text { Delta } \\
\text { Amacuro }\end{array}$ & $\begin{array}{c}\text { British } \\
\text { Guiana }\end{array}$ & Suriname & $\begin{array}{l}\text { French } \\
\text { Guiana }\end{array}$ & Amapa \\
\hline about 80 & 4 & - & 3 & 2 & 1 & 1 \\
\hline about 170 & $7(4)$ & - & $5(1)$ & 3 & $5(1)$ & 1 \\
\hline 6 & $3(2)$ & - & $3(1)$ & 2 & - & - \\
\hline about 15 & 2 & 1 & 2 & 2 & 2 & 1 \\
\hline about 160 & $5(1)$ & 1 & 2 & 2 & 3 & 1 \\
\hline about 35 & $3(1)$ & - & 1 & 2 & 2 & 1 \\
\hline about 35 & $10(5)$ & - & $8(3)$ & 2 & 4 & 1 \\
\hline about 6 & $1 ?$ & - & - . & $1 ?$ & $1 ?$ & - \\
\hline about 45 & 1 & - & 1 & - & - & - \\
\hline 1 & $1(1)$ & - & 一 & $1(1)$ & - & - \\
\hline 12 & $2(1-2)$ & 一 & - & 1 & $2(1)$ & - \\
\hline 10 & 2 & 一 & 1 & $1-2$ & 2 & - \\
\hline about 75 & $2(1)$ & - & $2(1 ?)$ & 1 & $1-2$ & - \\
\hline 2 & $2(2)$ & - & $2(2)$ & 一 & - & - \\
\hline \multirow[t]{2}{*}{1} & 1 & 一 & 1 & - & - & - \\
\hline & $45-46(18-19)$ & 2 & $31(7-8)$ & $19-21(1)$ & $22-24(2)$ & 6 \\
\hline
\end{tabular}

in the West Indies nearly all are restricted to the higher parts of the larger islands. Some species that are resistant to drought occur on the small islands. It is not possible to say much in general about the distribution on the continent, because very few data are available. In some regions the maps indicate rather the distribution of collectinggrounds than of the species. 
Habit AND Ecology

The majority of the Gesneriaceae inhabit tropical rainforests. Most species are terrestrial: some are epiphytic, but occur not rarely also in terrestrial habitats. As far as known they prefer an acid or oligotrophic substrate. Many terrestrial species prefer a granitic subsoil, others may also occur on sandy or lateritic soils, on sandstone, or on ferrite; not one of the collectors reported a clayey substrate. Although they frequently grow in moist places, they may have structures for water-storage: tubers, succulent stems or leaves.

The habit of the Gesneriaceae is most diverse. Small delicate sappy herbs occur side by side with thick-leaved trees. Most herbaceous species are terrestrial, and some of these have tubers or thickened rhizomes with annual stems; others creep with stolons or are scandent and attach themselves with adventitious, sometimes specialized adhesive roots. The shrubby species (mostly subshrubs) usually have only a few little-branched erect or climbing, sometimes liana-like stems. Most climbers are indifferently epiphytic or terrestrial. The true epiphytes which are only exceptionally terrestrial are almost all subshrubs with erect, scandent, or pendulous branches, depending on the circumstances. Owing to their inaccessibility the data on these plants are scanty. Most of them were taken along riverbanks where they occur on lower branches.

\section{Distribution of The genera (Table, p. 324-325)}

As far as our present knowledge goes, it seems that many genera have a high species concentration in Colombia. For instance, in Besleria, the only large genus that has recently been revised, 50 out of a total of 170 species occur in Colombia and 40 of those are only known from there. Other instances are presented by the medium-sized or large genera Alloplectus, Columnea, Episcia, and Kohleria. On the other hand the genera occurring mainly or exclusively in the Guayana Highlands or in Guiana are much poorer in species: Chrysothemis (4 out of 6 species) and Tylosperma (monotypic) in the Guayana Highlands, Rhoogeton (2 species) in the Guayana Highland part of British Guiana, Lembocarpus ( 1 species) in the mountains of Dutch Guiana.

In Guiana as defined in the present study there are 15 genera of Gesneriaceae among which the two endemics mentioned just before. Most of the non-endemic genera occur throughout the greater part of the Western Hemisphere, though only one, Rechsteineria, attains both the northern and the southern limit of the range.

\section{Distribution of THE SPECIES}

As only 45 species of Gesneriaceae are known with certainty from Guiana, but little can be said in general about their distribution. Nevertheless it may be useful to summarize what is known of their areas, as these data are scattered through the taxonomic part of the present study.

The species can be arranged in groups with a roughly similar distribution. One of them (6.4) occurs almost throughout tropical America 


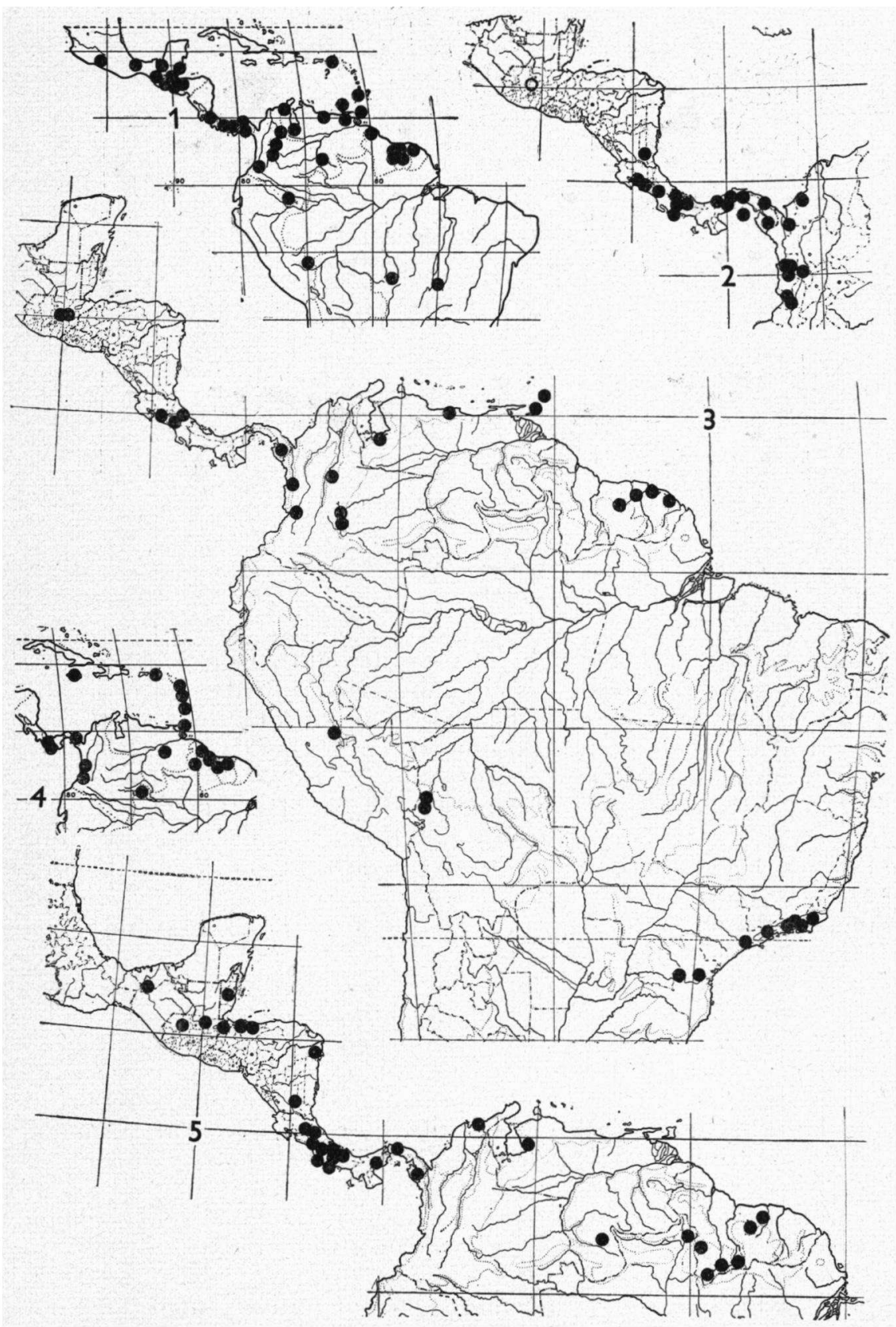

Map 1. Drymonia serrulata; Map 2. Chrysothemis friedrichsthaliana; Map 3. Napeanthus; Map 4. Chrysothemis pulchella; Map 5. Besleria laxiflora. 


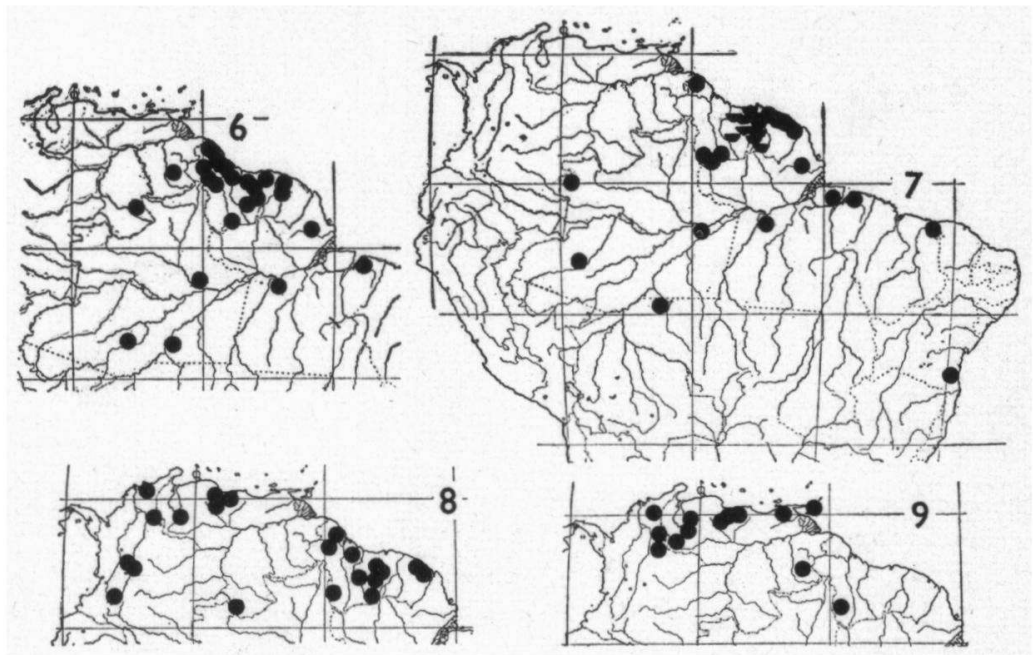

Map 6. Codonanthe calcarata; Map 7. Alloplectus coccineus, complete dots: var. coccineus, half dots: var. fusco-maculatus; Map 8. Rechtsteineria incarnata; Map 9. Kohleria hirsuta.

(Map 1), one (7.10) throughout tropical South America. Three (1.2, 3.1, 5.5) have a more or less circum-caraibic distribution (e.g. Map 4). Four species are restricted to the continental part of tropical America adjacent to the Caraibic region, two $(2.4,4.2)$ occurring from Central to northern South America (see Map 5), two others $(5.2,5.4)$ with a gap in Venezuela and Colombia. Five species $(1.3$, $5.1,7.3,9.1,13.1$ ) occur from Colombia to Guiana (Map 8), some of which have a more or less Andean distribution within this area (Map 9). Four species $(1.1,4.1,6.1,12.1)$ occur in Guiana and the Amazon basin (Maps 6 and 7). One species (2.1) is only known from Guiana and Bahia. Ten or eleven species $(1.4,2.7,3.3,5.3,7.2,7.5$, $7.9,13.2,14.1,14.2,15.1)$ are confined to the Guayana Highlands four or five of which are endemics of British Guiana $(3.3,7.9,13.2$, 14.1, 14.2). Eighteen or nineteen are endemics of Guiana: one of these occurs from British Guiana to Amapá with a gap in Suriname (2.2), one is known from the three Guianas (7.4), one from British and French Guiana, presumably still to be discovered in Suriname, one from British and Dutch Guiana (3.2), and two or three (6.2, $7.11,11.2)$ from Dutch and French Guiana. Three $(2.8,7.1,7.6)$ are restricted to British Guiana (i.e. not in the Guayana Highland part of that country), one (10.1) to Suriname, and two $(2.6,11.1)$ to French Guiana.

It is evident that the endemism is comparatively high, and that the greatest concentration of species and endemics in the region is found in British Guiana. 


\section{REVISION OF GHRYSOTHEMIS (TUSSACIA)}

\section{HisTORY}

This small genus was first described by ReichenBach (1829, p. 28) as Tussaca with Besleria pulchella as type species. Later WALPERS (1847, p. 740) citing ReIchensach published the combination $T$. pulchella as Tussacia; this spelling was maintained by subsequent authors. Independently Decaisne (1849, p. 242) described Chrysothemis and designated the same species as type species. As the name Tussaca was earlier used by RAFINESQUE (1814, p. 42) for a genus of the Orchidaceae, Chrysothemis is the valid name as stated by Moore (1954, p. 86)

Most additional species have been described as Tussacia.

\section{Geographical Distributton}

Chrysothemis occurs in the West Indies, Central and northern South America. Three species, $C$. dichroa, $C$. semiclausa, and $C$. villosa, are only known from the Guayana Highlands. The fourth, C. pulchella, which occurs in the West Indies, Panama and northern South America, is the most widespread (Map 4, p. 327). C. friedrichsthaliana occurs in Central America and western Colombia (Map 2, p. 327). The sixth, $C$. rupestris, is restricted to British Guiana and Suriname.

\section{RELATIONSHIP WTTH OTHER GENERA}

Within the subtribe of the Columneinae of the tribe Columneae, Chrysothemis is the most clear-cut genus. It is related to the section Skiophila of Episcia (of the same subtribe) and to the section Sinningia of Sinningia (tribe Sinningieae). From Episcia it differs mainly by its tubers and united sepals. Points of similarity are the habit, fruit, and seeds. Sinningia differs by its large corolla which is usually more than $3 \times$ as long as the calyx, its mostly inferior ovary, and its often less united sepals; it agrees in its tubers and sometimes in its habit.

Chrysothemis Dcne., Rev. Hortic. Sér. 3. 3: 242. 1849; op. cit. 4: 381. 1850; H. E. Moore, Baileya 2: 86. 1954.

Homotypic synonyms: Tussaca Rchb., Icon. Bot. Exot. 1: 28, t. 41. 1827 .

Tussacia Rchb. in Mösler, Gemeinnütz. Handb. Gewächsk. 2nd ed. 1: p. XXXVI. 1827 (not in 3rd ed. 1833); Conspect. Regn. Veg. 125. 1828; Bartling, Ord. Nat. Pl. 175. 1830; Reichenbach, Repert. Herb. sive Nom. Bot. Gen. Pl. 1: 120. 1841; Steudel, Nom. Bot. 2nd ed. 2: 725. 1841; Heynhold, Nom. Bot. Hort. 2: 747. 1846 (= Alph. Syn. Aufz.); Bentham, Lond. Journ. Bot. 5: 360. 1846; Walpers, Repert. 6: 740. 1847; Ann. 2: 1076. 1852; op. cit. 5: 432. 1858; Oersted, Centralamer. Gesn. 39. 1858; Grisebach, Fl. Br. W.I. Isl. 463. 1861 (“1864"); Hanstein, Linnaea 34: 245. 1865; Bentham \& Hooker, Gen. Pl. 2: 1006. 1876; Fritsch in Engl. Prantl, 
Nat. Pflanzenf. 4 (3b): 167. 1894 (“1895”); Duss, Fl. Ant. fr. 431. 1897; Urban, Symb. Ant. 2: 355. 1901; Lemée, Dict. 6: 765. 1935; Bailey, Hortus Second 745. 1947; Chittenden, R.H.S. Dict. Gard. 4: 2172. 1951; Morton in Fl. Trin. and Tob. 2 (5): 303. 1955.

Not Tussaca Rafinesque, Précis Découv. Somiol. 42. 1814; nor Schmalz ex Steudel, Nom. Bot. 859. 1821; nor Willdenow ex Schultes et Schultes, Syst. Veg. 7: p. X. 1829; nor Tyssacia Reichenbach ex Steudel, Nom. Bot. 2nd ed. 2: 727. 1841.

Terrestrial herbs with tubers, $0.05-2.00 \mathrm{~m}$ high. Stems succulent, erect, branched or not, mostly with spreading adventitious roots at the base. Leaves opposite, those of a pair equal or subequal, petiolate; blade dark green above, paler green or purple beneath, elliptic or oblong-elliptic to oblong-lanceolate, about $2-3 \times$ as long as wide, acuminate at the apex, crenate-serrate or serrate; midrib and veins prominent beneath; ends of primary veins arcuate along the margin; secondary veins reticulate. Inflorescence axillary, umbellate or rarely compound-umbellate, much shorter than the leaves, with some small bracts. Bracts leafy, oblong, lanceolate, or linear, puberulous on both sides. Calyx red, green, or yellow, campanulate or infundibuliform, wide in proportion to the corolla; sepals united for most of their length. Corolla yellow or orange, red-spotted or -lined on the limb and in the throat or only in the throat, nearly trumpet-shaped, erect in the calyx, $1 \frac{1}{4}-2 \frac{1}{2} \times$ as long as the calyx, not spurred, not or hardly gibbous at the base, not ventricose, glabrous or hairy outside, inside with a ring of hairs near the insertion of the stamens and glandular hairs in the throat; tube cylindric; limb spreading, horizontal on the tube; lobes 5, subequal, rounded or nearly so, entire. Stamens 4, didynamous, included; filaments glabrous, contorted or not, all connate at the base, inserted on the base of the corolla, anthers orbicular, 1-1.5 mm in diam., coherent or not; cells parallel, discrete, dehiscent throughout by a longitudinal split. Staminode none. Ovary superior, pubescent, ovoid; style mostly glabrous, erect, about as long as the stamens or shorter; stigma bilobed, pubescent with glandular hairs. Disk gland usually one, dorsal, mostly bilobed, sometimes four. Placentae bilamellate ovuliferous on the inner surface only. Fruit a capsule, included in the calyx, globose, bivalved, obtuse at the apex. Seeds dark brown, shining, ellipsoid, obliquely striate, mostly about $2 \times$ as long as wide, mucronate at the base, with endosperm, borne on long white translucent funiculi with small arilli. Embryo straight.

Type species: $C$. pulchella (Donn ex Sims) Dcne.

Distribution: 6 species in the West Indies, Central and northern South America.

Key to the species:

1. Calyx not angled, red, infundibuliform or campanulate, serrate at the margin or with 5 broadly triangular lobes which are up to half as long as wide; corolla mostly $2-2 \frac{1}{2} \times$ as long as the calyx

Calyx 5-angled, red, green, or yellow, campanulate, $\dot{5}$-lobed, 
lobes mostly about as long as wide; corolla $1 \frac{1}{4}-2 \frac{1}{2} \times$ as long as the calyx . . . . . . . . . . . . . . . . . .

2. Leafblades not decurrent into the petiole; calyx serrate or obscurely 5-lobed. British Guiana, Suriname

Leafblades decurrent into the petiole; calyx 5-lobed, lobes broadly triangular, about half as long as wide. BRITISH Gulana . . . . . . . . . . . . 6. C. villosa

3. Calyx red, campanulate, not inflated, approximately rounded at the base, 5-angled, seemingly winged or not, inside mostly glabrous or puberulous throughout; corolla $1 \frac{1}{2}-2 \frac{1}{2} \times$ as long as the calyx . . ... . . . . . . . . . .

Calyx red, green, or yellow, inflated, mostly subcordate at the base, strongly 5 -angled, seemingly winged, inside puberulous above; glabrous below; corolla usually $1 \frac{1}{4}-1 \frac{1}{2} \times$ as long as the calyx . . . . . . . . . . . . . . . . . .

4. Leafblades not decurrent into the petiole; calyx lobes broadly triangular, about half as long as wide, acute. GuAyana Highlands ............ . 5. C. semiclausa

Leafblades decurrent into the petiole; calyx lobes about as long as wide, acuminate. West Indies, Panama to Suriname

3. G. pulchella

5. Calyx red; corolla outside villose above. Guayana Highlands

Calyx green or yellow; corolla outside glabrous or nearly so. Central America and western Colombia

2. C. friedrichsthaliana

1. C. dichroa Lwbg., sp. nov.

Fig. 1D, p. 332

Herba terrestris. Caulis apice puberulus. Folia breviter petiolata laminis ellipticis vel oblongo-lanceolatis. Inflorescentia axillaris umbellata 2-4-flora. Pedunculus solitarius puberulus bracteis nonnullis suffultus. Calyx campanulatus inflatus. Corolla ecalcarata basi gibbosa haud ventricosa. Stamina quattuor didynama inclusa filamentis glabris contortis basi omnibus inter se connatis basi corollae insertis. Antherae orbiculares cohaerentes (?) loculis parallelibus discretis fissura longitudinali totam per longitudinem dehiscentibus. Staminodium nullum. Ovarium pubescens ovoideum. Stylus glaber. Stigma bilobum. Disci glandula singula dorsalis rectangularis emarginata glabra. Placentae duae bilamellatae lamellis facie interiore solum ovuliferis.

Type: Colombia: Vichada, near Maipures, left bank Orinoco R., Spruce 3612 (K, holotype, photographs: U, WAG; isotypes: BM, BR, P, W, photographs of $\mathrm{W}$ sheet: F, US).

Plant 10-30 cm high. Stems red. Leaves: petiole puberulous, $3-15 \mathrm{~mm}$ long; blade dark green above, paler beneath, $2-3 \frac{1}{2} \times$ as long as wide, $6.5-24 \times 2.5-11$ $\mathbf{c m}$, the basal ones often smaller, succulent, papyraceous or membranaceous when dry, acuminate, obliquely cuneate at the base, decurrent into the petiole or not, crenate-serrate, strigillose above, puberulous beneath. Inflorescence: peduncle $1.5-5 \mathrm{~cm}$ long. Bracts oblong-lanceolate, $2-3 \frac{1}{2} \times$ as long as wide, $4-9 \times 2-4 \mathrm{~mm}$, 


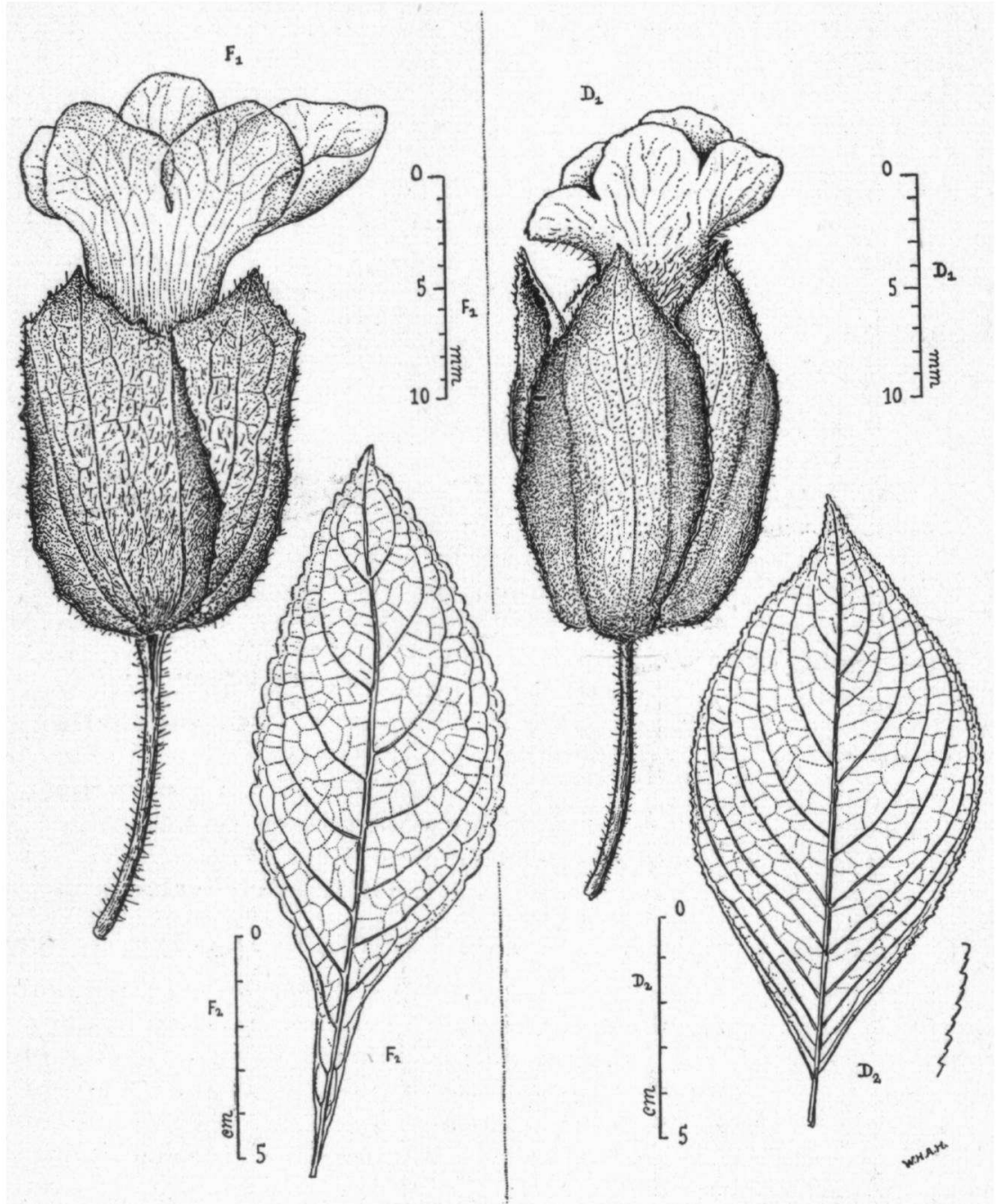

Fig. 1. $D_{1}$. flower and $D_{2}$. leaf of Chrysothemis dichroa (Spruce 3612); $F_{1}$. flower and $\mathrm{F}_{2}$. leaf of $C$. friedrichsthaliana (Allen 2674).

acuminate, entire, puberulous on both sides. Pedicels puberulous, 10-20 mm long. Calyx red, strongly 5-angled, subcordate at the base, 17-22 $\times 11-13 \mathrm{~mm}$, puberulous outside, inside puberulous only on the lobes, glabrous; sepals united for most of their length; lobes triangular, as long as wide, 5-7 mm long, acuminate, obscurely dentate. Corolla orange or yellow, with two rounded red (?) spots in the throat (seen on Spruce 3612), erect in the calyx, $1 \frac{1}{-1} \frac{1}{2} \times$ as long as the calyx, 22-25 mm long, 4-6 mm wide, slightly contracted above the gibbosity, outside villose above, glabrous below, inside with a ring of hairs near the insertion of the stamens and glandular hairs in the throat; tube cylindric, about as long as the calyx; limb spreading, large, 14-15 $\mathrm{mm}$ wide; lobes subequal, nearly rounded, entire, 6-7 $\times 8-9 \mathrm{~mm}$. Anthers $1.2 \mathrm{~mm}$ in diam. Ovary $3 \times 2 \mathrm{~mm}$. Fruit, seeds, and tubers not seen. 
Distribution: Guayana Highlands.

Ecology: on rocks in forests.

Colombia: Vichada: near Maipures, left bank Orinoco R., Spruce 3612 (K, holotype, isotypes: BM, BR, P, W); ibid., Chaffanjon $330(\mathbf{P})$.

Venezurla: Amazonas: Puerto Ayacucho, alt. about $100 \mathrm{~m}$, Holt \& Blake 817 (US); Upper Orinoco R., Puerto Zamuro, Gaillard 12 (P).

2. C. friedrichsthaliana (Hanst.) Moore, Baileya 2: 87. 1954; African Violets, Gloxinias, and their relatives 192, 272, f. 27. 1957.

Fig. 1F, p. 332

Basionym: Tussacia friedrichsthaliana Hanst., Linnaea 34: 337. 1865; Pittier, Prim. Fl. Costar., Anales Inst. Fisico-Geogr. Nac. 8: 186. 1898; Morton, Field Mus. Publ. Bot. 18: 1186. 1938; Johnston, Sargentia 8: 277. 1949.

Type: Guatemala: Friedrichsthal 1252 (W, holotype, photographs: U, US, WAG; isotype: W, photographs: U, WAG).

Leafblades decurrent into the petiole. Calyx green or yellow, campanulate, pentagonal, inflated, mostly subcordate at the base, 11-22 (mostly 14-20) × 7-17 mm, puberulous outside, inside puberulous above, glabrous below; lobes about as long as wide, acuminate, dentate. Corolla yellow or orange, with red longitudinal lines in the throat, 11-1 $\frac{1}{2}$ (sometimes up to 2) $\times$ as long as the calyx, 19-28 $\mathrm{mm}$ long, outside glabrous or nearly so. Style short, mostly glabrous, $5-8 \mathrm{~mm}$ long.

Plant 5-50 (mostly about 20-40) $\mathrm{cm}$ high. Tuber about $2-3.5 \mathrm{~cm}$ in diam. Stems green, rarely with some short red lines, one or several on one tuber. Leaves short-petiolate; blade dark green above, paler beneath, elliptic, $2-3 \times$ as long as wide, mostly about $10-20 \times 4-8 \mathrm{~cm}$, up to $30 \times 13 \mathrm{~cm}$, succulent, membranaceous when dry, irregularly crenate-serrate, pilosulous above, puberulent beneath, especially on the midrib and veins. Inforescence many-flowered. Peduncle puberulous, 5-45 mm long. Bracts lanceolate, about $5 \times$ as long as wide, $2-15 \times 0.5-3 \mathrm{~mm}$. Pedicels puberulous, 10-40 mm long. Corolla 5-6 mm wide, not gibbous at the base; limb 10-21 mm in diam.; lobes 4-7 $\mathrm{mm}$ in diam. Filaments not contorted or only at the apex; anthers coherent in pairs, 1-1.2 mm in diam. Ovary 2-4 $\times 2-3$ $\mathrm{mm}$. Disk gland one, mostly bilobed, sometimes entire or several-lobed. Capsule pubescent, $7-10 \mathrm{~mm}$ in diam. Seeds $1 \frac{1}{2}-3$ (mostly about 2) $\times$ as long as wide, $0.3-0.6 \times 0.2-0.25 \mathrm{~mm}$.

Distribution: Central America and western Colombia.

Ecology: Usually on wet rocks in mostly open forests or in clearings; alt. 0-900 m.

Guatemala: Friedrichsthal 1252 (W, 2 sheets, type), anno 1841 (K).

Nigaragua: between R. Punta Gorda, Alemán, and Zapote, Shank \& Molina 4958 (US).

Costa RrcA: vic. of Los Chiles, R. Frio, Alajuela, Holm \& Iltis 816 (US); Basin of El General, San José, Skutch 4951 (MO, US); banks of R. Jesus Maria, road from Puntarenas, Pittier 466 (BR, US), 467 (C); vic. of Cascajal, near Machuca R., Holm \& Iltis 252 (BM, G, NY, U, US).

Panama: Bocas del Toro: Changuinola Valley, Dunlap 352 (BM, US); region of Almirante, Proctor Cooper 142 (NY); vic. of Chiriquí Lagoon, von Wedel 789 (MO, US), 1130 (MO), 1458 (MO, US), 2509 (MO, US), 2621 (MO, US).

ChIRIQuf: vic. of San Bartolomé, Peninsula de Burica, Woodson \& Schery 878 (MO, NY, US).

Colón: vic. of Porto Bello, Pittier 2488 (US); near Fató, Nombre de Dios, Pittier 4085 (US). 
C.Z.: vic. of Salamanca Hydrographic Station, R. Pequení, Woodson, Allen \& Seibert 1614 (MO, NY, US); vic. of Las Cruces, Seibert 569 (US); Barro Colorado Island, Kenoyer 536 (US); ibid., Killip 40038 (US); ibid., Standley 40952 (US, BM), 41121 (US, K); ibid., Dodge 3469 (GH); vic. of Madden Dam, near R. Chagres, Seibert 556 (MO, K, NY, US); Azote Caballo Road near Madden Dam, Allen 2647 (MO, US); Gamboa, Riley 301 (BM, K).

Panama: Capira, Bro. Paul 134 (US); vic. of Alhajuela, Chagres Valley, Pittier 3490 (US); between Pacora and Chepo, Woodson, Allen \& Seibert 1642 (MO, NY, US).

San Blas: Perme, Proctor Cooper III 258 p. p. (NY).

DARIEn: Cana and vic., R. S. Williams 939 (NY); Headwaters R. Chico, Allen 4594 (MO, G).

SAN Još Iszand: Erlanson 493 (NY, G, US), 574 (US); ibid., Johnston 141 (US, FI).

Colombia: Bolfvar: Boca Verde, on R. Sinu, Cacaotala, Pennell 4588 (NY).

Antioquia: South of Turbo, R. Chiado, Haught 4647 (US); Gorge of R. Chigorodó, Haught 4726 (US); Punta Piedra, Haught 4824 (US); near Villa Arteaga, Hodge 6976 (US).

Chocó: Bahia de Solano, between Quebrada Seca and La Chorrera, Fernández 325 (US); between La Oveja and Quibdó, Archer 1703 (US); Quibdó, R. Atrato, Archer 2107 (US); La Concepción, East of Quibdó, Archer 2210 (US); near junction of R. Condoto and R. San Juan, Killip 35079 (US, BM); Corcovado Region, Upper R. San Juan, Killip 35280 (US); Mandinga, vic. of Andagoya, R. San Juan, Garcia-Barriga 11528 (US); Juntos de Dagua, Novita, Triana 4167 (BM).

Caldas: Santa Cecilia, von Sneidern 5189 (US).

Valle del Cauca: vic. of Buenaventura, Killip 33232 (US); R. Anchicayá, Køie 4766 (C); ibid., Quebrada del Danubio, Cuatrecasas 13722 (US); R. Calima, Quebrada de La Brea, Schultes \& Villarreal 7394 (US); Cordoba, Dagua Valley, Killip 5077 (GH, NY, PH, US); Cisneros, Dagua Valley, Killip 11436 (NY, PH), 35535 (US, BM).

Cauca or Valle del Cauga: R. Dagua, Lehmann 8423 (K).

Cauca: Lower R. Dagua, Lehmann, April 1885 (K).

Dept.? "Chocó and Barbacoas", Triana 2539 (G, P).

3. C. pulchella (Donn ex Sims) Dcne., Rev. Hortic. Sér. 3. 3: 242. 1849; op. cit. 4: 382. 1850; H. E. Moore, African Violets, Gloxinias, and their relatives $192,272.1957$.

Basionym: Besleria pulchella Donn [, Hort. Cantabr. 4th ed. 140. 1807 (nomen nudum)] ex Sims, Bot. Mag. 28: t. 1146.1808 (descr. et icon.); D. Donn, op. cit. 5th ed. 149. 1809; op. cit. 6th ed. 166. 1811; James Donn, op. cit. 7th ed. 179. 1812; Pursh, op. cit. 8th ed. 197. 1815; op. cit. 9th ed. 197. 1819; Lindley, op. cit. 10th ed. 230. 1823; op. cit. 11th ed. 239. 1826; Sinclair, op. cit. 12th ed. 285. 1831; Aiton, Hort. Kew. 2nd ed. 4: 21. 1812; Loddiges, Bot. Cab. 11 : t. 1028. 1825; Sprengel, Syst. Veg. 2: 840. 1825; Loudon, Encycl. 354. 1829; Maycock, Fl. Barb. 242. 1830; D.C., Prod. 7: 539. 1839; A. Richard in Sagra, Fl. Cubana 3 (Fanerogamia 2): 72. 1853.

Type: Cult., Woodford, Springwell, Hertfordshire, Aug. 1808 (BM). Homotypic synonyms: Tussacia pulchella (Donn ex Sims) Rchb. ex Walpers, Repert. 6: 740.1847 (see also Reichenbach, Icon. Bot. Exot. 1: 28, t. 41. 1827); Oersted, Centralamer. Gesn. 39, t. 10. f. 14-19. 1858; Grisebach, Fl. Br. W.I. Isl. 463. 1861 ("1864"); Cat. Pl. Cub. 201. 1866; Hanstein, Linnaea 34: 337. 1865; author?, Kew Bull. 1893: 267; Duss, Fl. Ant. fr. 431. 1897; Urban, Symb. Ant. 2: 355. 1901; L1. Williams, Expl. Bot. Guyana Venezolana 385. 1942; 
Bailey, Hortus Second 745. 1947; Chittenden, R. H. S. Dict. Gard. 4: 2172 , with fig. 1951; Morton in Fl. Trin. and Tob. 2 (5) : 303: 1955. Episcia pulchella (Donn ex Sims) Mart. ex G. Don, Gen. Syst. 4: 656. 1838 (see also Martius, Nov. Gen. 3: 45. 1829); D. Dietrich, Syn. 3: 580. 1843; P. N. Donn, Hort. Cantabr. 13th ed. 408. 1845; Seemann, Voy. H. M. S. Herald 187. 1854; Hartinger, Parad. Vindob. $1: 39$, t. 66.1860 ("1848-1860").

Skiophila pulchella (Donn ex Sims) Hanst., Linnaea 26: 207.1853.

Heterotypic synonyms: Chrysothemis aurantiaca Dcne., Rev. Hortic. Sér. 3. 4: 381. 1850; G. Don in Loudon, Encycl. suppl. 2: 1402. 1855. Lectotype: Cult. Hort. Paris, Aug. 1850 (P).

Tussacia woodsoni Morton, Ann. Mo. Bot. Gard. 26: 308.1939. Type: Panama: prov. Chiriquí, between R. Chiriquí and Remedios, Woodson, Allen \& Seibert 1195 (US, holotype, isotypes: NY, US; photographs of NY sheet in U and WAG).

Misapplied name: Besleria melittifolia Drapiez, Herb. Amateur 6: t. 438. 1833, not L.

Leafblade decurrent into the petiole. Calyx orange or red, campanulate, 5-angled, $10-18 \times 5-11 \mathrm{~mm}$, puberulous or villose outside, especially below, glabrous or (rarely only apically) minutely puberulous inside; lobes acuminate, dentate. Corolla with red longitudinal lines on the limb and in the throat, $1 \frac{1}{2}-2 \frac{1}{2}$ (mostly about 2 ) $\times$ as long as the calyx, 16-30 mm long, outside more or less white-sericeous above, glabrous below.

Plant $10-90 \mathrm{~cm}$ high. Tuber about $2-3 \mathrm{~cm}$ in diam. Stems green, sometimes partially purple or with some short red lines, puberulous, often glabrescent. Leaves: blade elliptic, ovate, or oblong-ovate, variable, $1 \frac{1}{2}-3 \frac{1}{2}$ (mostly about 2 ) $\times$ as long as wide, up to $30 \times 12 \mathrm{~cm}$, membranaceous or papyraceous when dry, sparsely strigillose to villose above, puberulous beneath, especially on the midrib and veins. Inflorescence: peduncle puberulous, $1-5 \mathrm{~cm}$ long. Bracts oblong, lanceolate, or sometimes linear, 2-10 (mostly 3-5) $\times$ as long as wide, 4-15 $\times 1-6 \mathrm{~mm}$, acuminate, entire or obsc urely serrate, puberulous on both sides. Pedicels puberulous, $1.5-2.5 \mathrm{~cm}$ long. Corolla $3-6 \mathrm{~mm}$ wide, somewhat gibbous at the base, slightly contracted above the gibbosity; limb $10-15 \mathrm{~mm}$ wide; lobes $3-8 \mathrm{~mm}$ in diam. Anthers all coherent or free, $1-1.2 \mathrm{~mm}$ in diam. Ovary $2-4 \times 2-3 \mathrm{~mm}$; style about as long as the stamens, glabrous, 7-11 mm long. Disk gland one, bilobed, rarely entire or several-lobed (no ventral or lateral glands seen). Capsule sparsely pubescent, $6 \mathrm{~mm}$ in diam.

Distribution: West Indies, Panama, Colombia, Venezuela, British Guiana, Suriname, and Brazil (Amazonas).

Ecology: On wet rocks in rainforests; alt. 0-300 m.

CuBA: near Habana, La Ossa (fide D.C., 1839, p. 539), no specimens seen.

JAMAica: St Elisabeth, Balaclava, Orcutt 2345 (BM, UC); Mandeville, Clover, Harris 12101 (NY, BM, GH, K, MO, US); from Miss Stewart of Liberty Hill, original from some miles Southeast, Britton 2553 (NY); Caley s.n. (BM); W.T. March 1185 (K, GH), 1333 (GOET). (In Jamaica naturalized according to W. T. Stearn, personal commun.).

ST. Thomas (not the island, according to Urban, 1901, p. 356): Eggers 349b, comm. Rensch (LE, M, P, W); Eggers 393 (GH), Nov. 1882 (US).

GuAdeloupe : Ravine Chaude, Duss 3384 (MO, NY, US); Trois Rivières, Stehlé 141 (S, US).

MARTINIQUe: Ravin of Mt. Pelée, Hahn 495 (P, BM, G); Camp Colson, between la Fontaine Didier et Absalon, Duss 328 (NY, US); Trinité, herb. Sieber s.n. 
(AWH); Belanger 365 (P), 776 (P), s.n. (P); Plée, anno 1820 (P); herb. Sieber s.n. (AWH).

St. Vincent: Guildingh, anno 1822 (K).

Grenada: Road to Grand Etang, Guildingh (?), July 1824 (GH).

Barbados: (fide MaYcock, 1830, p. 242), no specimen seen.

Antilles: herb. Persoon s.n. (L).

Panama: Ghiriquí: R. Chiriquí to Remedios, Woodson, Allen \& Seibert 1195

(NY, US, 2 sheets, type of Tussacia woodsoni); vic. of San Felix, Allen 3652 (MO, BM, BR, G, U); ibid., Pittier 5200 (US).

Veraguas: Isla de Uva, Contretas Group, Pittier 5111 (US); Coiba Island, Collenette 465 (BM, K, US).

San Blas: Perme, Proctor Cooper III 258 p. p. (US).

Sin. loc.: Hayes 188 (BM, K); herb. Bernhardi s.n. (MO); Duchassaing, anno 1850 (GOET, P).

Colombia: Chocó: Cabo Corrientes, Seemann s.n. (BM).

VAlle del CaUca: Porreto, R. Dagua, Buenaventura, Lehmann 4717 (K, US).

Cauga: near R. Dagua, Lehmann 2631 (BM).

Colombia?: herb. Triana, rec. 1891 (BM).

Venezuela: Bolfvar: Middle Caura R., Salto de Para, Ll. Williams 11460 (F, US).

Trinidad: Blue Basin, near Port of Spain, Friend 128 (NY); ibid., Graham 513

(NY); ibid., Went 1020 (U); Slope of Mt. Tocuche, Othmer 455 (M); Aripo Savanna at Waller Field, Howard 10345 (US); Oropuche Road via Valencia, Broadway 6172 (K), 6925 (BM, K, MO, S) ; Cumaca Road, Heights of Oropuche, Simmonds 274 (US); Maraval, Crueger, anno 1847 (or no. 47) (K); Diego Martin Valley, Simmonds 207 (US); Woodbrook, Broadway, 4 Sept. 1932 (BM); Balandra Bay, Britton, Britton \& Hazen 395 (NY, GH, US); Fendler 509 (BM, K); herb. Kuntze 624 (NY, US); herb. Splitgerber, annis 1837-1838 (L); Warming, 28 Feb. 1891 (C).

ToBAGo: between Parlatuvier and Bloody Bay, Sandwith 1895 (K).

British Guiana: Pakaraima Mts., Wenamu R., Davenport $8(\mathrm{~K})$; Pomeroon distr., Moruka R., de la Cruz 4593 (NY, F, GH, MO, PH, US); Great Falls, Demerara R., Jenman 3990 (K, NY, U); spontaneous in Bot. Garden, Jenman $5608(\mathbf{K})$; Drake, comm. Campbell, June $1871(\mathrm{~K})$.

SuRINAmE: Avanavero Falls, Kabalebo R., Corantijn trib., B. W. 4602 (U); ibid., Florschütz 2213 (U); ibid., Pulle 437 (U); Paramaribo, near Kombé, Pulle H 13 (U).

Brazil: Amazonas: San Gabriel, near R. Tiquié, right bank R. Vaupés, Spruce 2407 (K, P).

Sin. loc.: Delessert, anno 1822 (G-DC); herb. J. E. Smith 1058.8 (LINN), 1058.9 (LINN).

Cult.: herb. J. E. Smith 1058.7 (LINN); Lady Bourne, 4 Dec. 1900 (K); herb. Gay, Chelsea Garden, 13 Sept. 1824 (K); Hort Paris, Aug. 1850 (P, lectotype of Chrysothemis aurantiaca); Hort. Kew., June 1858 (K); Caum, from Panama, Beltsville, Md. (US, MO); Woodford, Springwell, Hertfordshire, Aug. 1808 (BM, holotype); Hort. Utrecht sin. dat. (U, L); ex herb. Hamburg (C); Hort. Boyton, anno 1825 (W); herb. Endlicher, Hort. Vienna (W); herb. Fenzl., Hort. Vienna (W); Hort. Vindob., Aug. 1824 (W); herb. Fischer s.n. (GOET); herb. Martens s.n. (BR); anonym., ante 1840 (U); anonym. (W. herb. 111006, 111074); herb. Baillon s.n. (P-BA).

Cult.?: herb. Gesner (?), anno $1895(\mathrm{~K})$; from South America, Shakespear s.n. (BM); anonym., anno 1823 (G); anonym. (BM).

\section{C. rupestris (Benth.) Lwbg., comb. nov.}

Figs. 2, p. 337; 3R, p. 339

Basionym: Tussacia rupestris Benth., Lond. Journ. Bot. 5: 363. 1846; Walpers, Repert. 6: 740. 1847; Schomburgk, Reisen, Br. Guian. 3: 972. 1848; Hanstein, Linnaea 34: 339. 1865; Morton, Bull. Torr. Bot. Cl. 75: 566. 1948.

Type: British Guiana: Kanuku Mts., Schomburgk s.n. (K, holotype, photographs: U, WAG). 


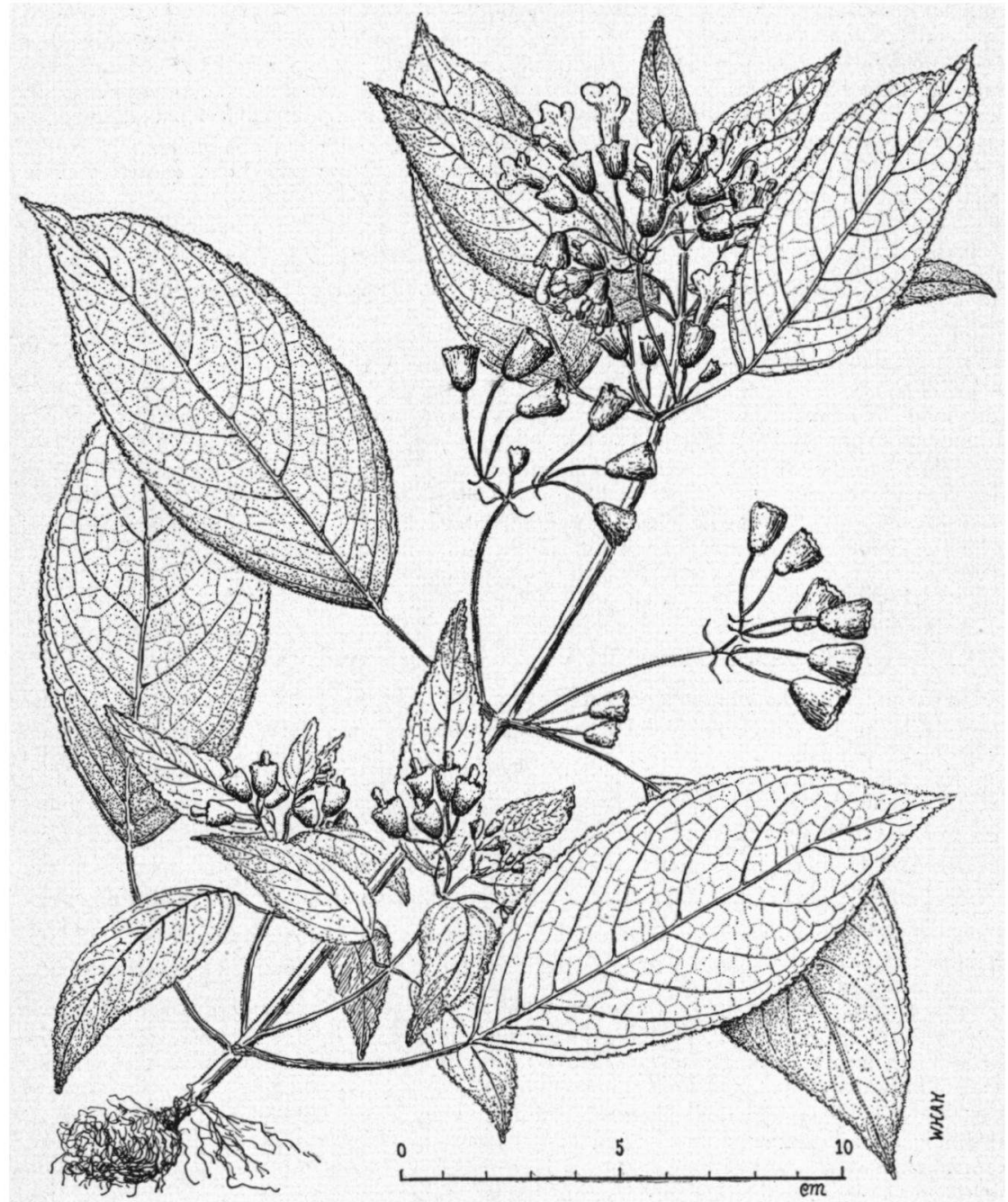

Fig. 2. Chrysothemis rupestris (Florschütz 1326).

Leafblade not decurrent into the petiole. Calyx orange or red, infundibuliform, 10-15 $\mathrm{mm}$ long, at the margin 6-12 $\mathrm{mm}$ wide, serrate or obscurely 5-lobed, puberulous outside, glabrous inside. Corolla $1 \frac{1}{2}-2 \frac{1}{2}$ (mostly 2 ) $\times$ as long as the calyx, 18-25 mm long, outside villose above, glabrous below.

Plant $5-200 \mathrm{~cm}$ high. Tuber about $2-3 \mathrm{~cm}$ in diam. Stems green or partially purple, sometimes with short red lines, puberulous at the apex, glabrescent. Leaves: petiole puberulous, $0.5-5 \mathrm{~cm}$ long; blade ovate or oblong-ovate, $1 \frac{1}{2}-2 \frac{1}{2} \times$ as long as wide, of very variable size, up to $24 \times 14.5 \mathrm{~cm}$, membranaceous or papyraceous when dry, cuneate or rounded at the base, sparsely strigillose above, puberulous beneath, especially on the midrib and veins. Inflorescence: peduncle puberulous, $1-6 \mathrm{~cm}$ long. Bracts linear, about $10 \times$ as long as wide, $5-12 \times 0.2-1$ 
mm, acuminate, entire, puberulous on both sides. Pedicels puberulous, $0.5-2.5$ $\mathrm{mm}$ long. Corolla red-lined or -spotted, 3-5 mm wide, obscurely gibbous at the base, slightly contracted above the gibbosity; limb 10-15 mm wide; lobes 4-5 $\mathrm{mm}$ in diam. Filaments not contorted or only at the apex; anthers coherent in pairs, 1-1.2 mm in diam. Ovary $2-4 \times 2-3 \mathrm{~mm}$; style mostly glabrous, sometimes with scattered hairs, 6-9 mm. Disk gland one, large, almost as long as the ovary, bilobed, rarely several-lobed. Capsule pubescent, $6-7 \mathrm{~mm}$ in diam. Seeds mostly about $2 \times$ as long as wide, $0.6-0.8 \times 0.25-0.3 \mathrm{~mm}$.

Distribution: British Guiana and Suriname.

Ecology: On wet, often granitic rocks among shrubs or in rainforests; alt. $0-400 \mathrm{~m}$.

British Guiana: Kanuku Mts., Schomburgk s.n. (K, holotype); Appun, rec. July $1866(\mathbf{K})$.

SURINAME: Corantijn R., Wonotobo Falls, Rombouts 119 (U); Wilhelmina Mts., on islet in rapid, B. W. 7226 (U); Tafelberg, Maguire 24762 (NY, U, US); Upper Gran Rio, Hulk 243 (U); ibid., Tresling 470 (U); Upper Tapanahoni R., near Mt. Teboe, Versteeg 760 (U); base of Voltzberg, Schulz 7611 (U); between Saramacca and Suriname Rs., between Pakka Pakka and Ebbatop, Florschütz $1326(U)$; near fall in trib. ck. of Kleine Saramacca R., Saramacca R., Florschütz $1400(\mathrm{U})$.

\section{C. semiclausa (Hanst.) Lwbg., comb. nov.}

Basionym: Tussacia semiclausa Hanst., Illustr. Hortic. 17: 140, t. 28. 1870; Chittenden, R. H. S. Dict. Gard. 4: 2172.1951.

Type: Brazil: Rio Branco, Serra Parima, Wallis, anno 1865; not seen, destroyed in Berlin?

Leafblades not decurrent into the petiole. Calyx red, pale green at the base, campanulate, 5-angled, hispid outside; lobes very short, broadly triangular, about half as long as wide, acute, serrate-dentate. Corolla yellow, red-lined on the limb and with two red spots in the throat, about $2 \times$ as long as the calyx, $25 \mathrm{~mm}$ long, hispid outside.

Stems green, with short red lines, purple at the base, sparsely pubescent at the apex, glabrescent. Leaves: petiole sometimes purple, short, widened at the base, sparsely pubescent; the bases of the petioles of a pair clasping the nodes of the stems; blade dark green above, paler beneath, red at the margin, elliptic, cuneate or rounded (?) at the base, serrate, ciliate, strigillose on both sides. Inflorescence: peduncle conspicuously longer than the petioles, sparsely pubescent: Bracts elliptic, acuminate, with a brown apex. Corolla dorsally gibbous at the base. Filaments slightly contorted; anthers connivent. Style about as long as the stamens. Disk glands four, surrounding the ovary, the dorsal one emarginate at the apex.

No specimens seen. Description based on Illustr. Hortic., icon. cit.

6. C. villosa (Benth.) Lwbg., comb. nov.

Fig. 3V, p. 339

Basionym: Tussacia villosa Benth., Lond. Journ. Bot. 5: 363. 1846; Walpers, Repert. 6: 740. 1847; Schomburgk, Reisen Br. Guian. 3: 972. 1848; Hanstein, Linnaea 34: 338. 1865.

Type: British Guiana: Kanuku Mts., Schomburgk s.n. (K, holotype, photographs: U, WAG).

Leafblade shortly decurrent into the petiole. Calyx red, pale green at the base, campanulate-infundibuliform, 10-17 mm long, at the limb 9-14 $\mathrm{mm}$ wide, 5-lobed; lobes broadly triangular, about half 


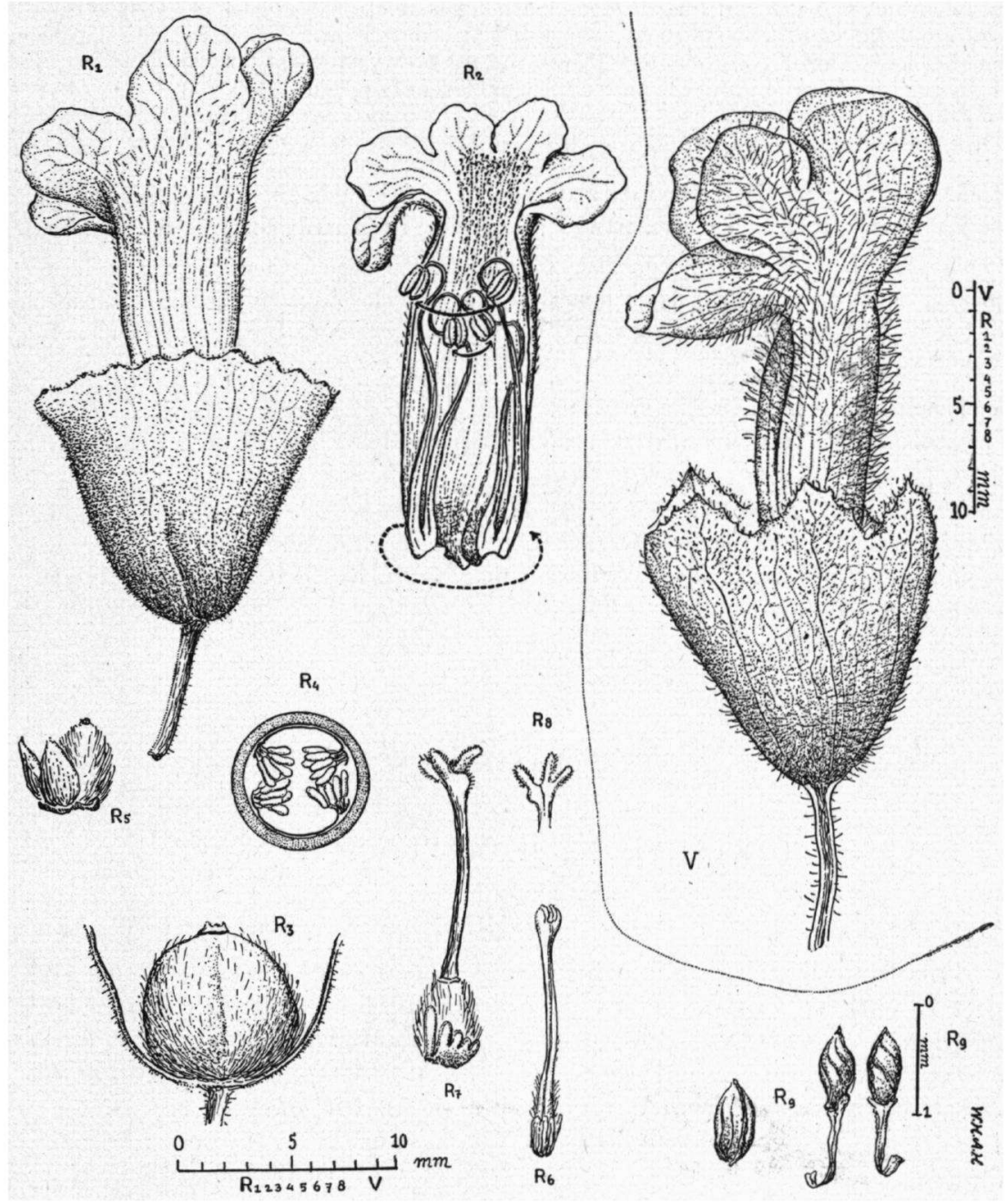

Fig. 3. R. Chrysothemis rupestris: 1. flower (Florschütz 1400); 2. opened corolla; 3. capsule; 4 . diagrammatic transverse section of ovary; 5 . ovary and disk gland; 6. pistil; 7. pistil with aberrant disk and stigma; 8. stigma of $7 ; 9$. seeds, two with funiculi $(2-9$, Florschütz 1326); V. flower of C. villosa (A. C. Smith 3579).

as long as wide, $2-4 \times 4-8 \mathrm{~mm}$, acute or shortly acuminate, dentate, puberulous outside, glabrous inside. Corolla $1 \frac{3}{4}-2 \frac{1}{2} \times$ as long as the calyx, 25-30 mm long, outside villose above, glabrous below.

Plant $5-40 \mathrm{~cm}$ high. Tuber about $2-3 \mathrm{~cm}$ in diam. Stems green, sometimes with short red lines, puberulous. Leaves: petiole puberulous, $1-3.5 \mathrm{~cm}$ long; blade ovate or oblong-ovate, 1-2 (mostly about $1 \frac{1}{2}$ ) $\times$ as long as wide, 9-24.5 $\times 4.5-14.5$ $\mathrm{cm}$, papyraceous when dry, crenate-serrate, strigillose above, puberulous beneath. Inflorescence: peduncle puberulous, $1-6 \mathrm{~cm}$ long. Bracts linear, $5-20 \times$ as long as wide, 6-15 $\times 0.5-2 \mathrm{~mm}$, acuminate, entire, puberulous on both sides. Pedicels 
puberulous, $0.5-2.5 \mathrm{~cm}$ long. Corolla with lobes deep red inside, or with yellow or orange lobes and red-spotted and -lined in the throat, 4-6 $\mathrm{mm}$ wide, gibbous at the base, slightly contracted above the gibbosity; limb 10-13 $\mathrm{mm}$ wide; lobes 4-6 mm in diam. Filaments not contorted or only at the apex; anthers coherent in pairs, $1.2-1.5 \mathrm{~mm}$ in diam. Ovary $2-4 \times 2-3 \mathrm{~mm}$; style glabrous, $9-10 \mathrm{~mm}$ long. Disk gland one, large, bilobed. Capsule pubescent, 6-8 $\mathrm{mm}$ in diam.

Distribution: British Guiana.

Ecology: On wet granitic (?) rocks in rainforests, along watercourses, at middle elevations.

British Guiana: Kanuku Mts., Schomburgk. s.n. (K, holotype); ibid., in drainage of Moku-moku Ck., Takutu trib., A. C. Smith 3579 (NY, F, G, K, MO, U, US); Komo Ck., Takutu R., F. D.-W. B. 569 (K), 635 (NY).

Excluded species:

Chrysothemis melittifolia (L.) G. Don in Loudon, Encycl. suppl. 2: 1402. 1855 = Episcia melittifolia (L.) Mart.

Chrysothemis venosa Dcne., Rev. Hortic. Sér. 3. 3: 242. $1849=$ Episcia melittifolia (L.) Mart.

Tussacia sanguinea (Pers.) Heynhold, Nom. Bot. Hort. 2: 747. $1846=$ Columnea sanguinea (Pers.) Hanst.

CHAPTER 5

\section{REVISION OF NAPEANTHUS}

\section{HisTORY}

The first description of this genus was made by Gardner (1843, p. 13) with the type species $\mathcal{N}$. brasiliensis $(=\mathcal{N}$. primulifolius $)$. Independently Karsten (1860, p. 97) described Marssonia in the Gentianaceae which later turned out to be a taxonomic synonym of Napeanthus. Most other species were correctly placed in the present genus.

\section{Geographical distribution}

Napeanthus occurs in tropical America, in Central and South America south to Peru, Bolivia, and southern Brazil (Map 3). All species have narrowly restricted areas in one or two countries, and the areas of most species do not overlap. Consequently there is no centre of species concentration.

$\mathcal{N}$. primulinus which has an actinomorphic corolla and androeceum occurs in Venezuela. $\mathcal{N}$. andinus, $\mathcal{N}$. primulifolius, and $\mathcal{N}$. rigidus where corolla and androeceum are pronouncedly zygomorphic occur towards the boundary of the area of the genus. Species with a slightly zygomorphic or actinomorphic corolla and four or five equal or four slightly didynamous stamens, for instance $\mathcal{N}$. subacaulis and $\mathcal{N}$. apodemus, inhabit regions closer to Venezuela. It is feasible that Venezuela is the centre of origin of the genus. 
Relationship OF THE SPECIES

The corolla is actinomorphic with five equal stamens or with one large staminode and four stamens, or zygomorphic with four mostly didynamous stamens and usually with one staminode. The more zygomorphic the corolla, the more unequal the stamens; there is an almost complete series between both extremes.

As $\mathcal{N}$. primulinus, the type species of Marssonia, differs mainly from most other species of $\mathcal{N}$ apeanthus by its entirely actinomorphic corolla, the present author does not maintain Marssonia as a distinct genus. In all other characters it agrees very well with Napeanthus.

$\mathcal{N}$. reitzii differs chiefly from the other species of $\mathcal{N}$ apeanthus by its angled seemingly inflated calyx, but by all other characters it is also a true Napeanthus. When the fruit is mature and the seeds have been shed the valves are also shed, but the placentae which are longitudinally cleft are persistent on the dried pedicel, conveying the impression of an axis, as they are erect and ovuliferous on both sides. The last has been observed on herbarium specimens of most species of this genus. This is probably what induced L. B. Smith to describe $\mathcal{N}$. reitzii in a new genus of Scrophulariaceae, Hatschbachia.

RELATIONSHIP WITH OTHER GENERA

Among the New World Gesneriaceae Napeanthus occupies an isolated position. According to FrITSCH (1894, p. 155) and BURTT it is related to certain Old World genera.

Napeanthus Gardn., Lond. Journ. Bot. 2: 13. 1843; D.C., Prod. 9: 274. 1845; Walpers, Repert. 6: 523. 1847; Bentham \& Hooker, Gen. PI. 2: 1018. 1876; Fritsch in Engl. Prantl, Nat. Pflanzenf. 4 (3b) : 155. 1894 ("1895"); Sitzungsb. Akad. Wiss. Wien 134, Abt. 1: 121. 1925; Solereder, Beih. Bot. Centralbl. 24 (2): 432. 1909; Lemée, Dict. 4: 642. 1932.

Type species: $\mathcal{N}$. primulifolius (Raddi) Sandwith (N. brasiliensis Gardn.).

Heterotypic synonyms: Marssonia Karst., Fl. Colomb. 1 : 97, t. 48. 1860; in Engl., Bot. Jahrb. 8: 361. 1887; Fritsch in Engl. Prantl, Nat. Pflanzenf., Nachtr. 3: 319. 1908; Solereder, Beih. Bot. Centralbl. 24 (2): 433. 1909; Lemée, Dict. 4: 319. 1932.

Type species: $\mathcal{N}$. primulinus (Karst.) Benth. et Hook. ex Jackson.

Hatschbachia L. B. Smith, Anais Bot. Herb. Barb. Rodr. 5 (5): 37. 1953.

Type species: $\mathcal{N}$. reitzii (L. B. Smith) Burtt.

Terrestrial perennial or sometimes annual (?) herbs, $5-45 \mathrm{~cm}$ high. Stems short or very short, rhizomatous, often woody. Leaves crowded at the apex, rosulate, sessile or subsessile, lanceolate, oblong-spathulate, oblanceolate, or linear-lanceolate, very variable, membranaceous or papyraceous when dry, remotely serrate, sinuate, or crenate-serrate, acute, acuminate, or obtuse at the apex, narrowed towards the base. Inflorescence axillary, cymose, subcymose, umbellate, subumbellate, or nearly paniculate, 1-many-flowered. Peduncle with some green leafy bracts when inflorescence several-flowered. Flower-buds nodding. 
Calyx usually green, campanulate; sepals 5 , equal, valvate in the bud, connate up to half of their length, oblong, lanceolate, or oblongtriangular, in flower spreading and inconspicuously veined, acuminate, entire, in fruit erect, enlarged, becoming thicker and conspicuously veined with longitudinal veins, connected here and there by cross veins. Corolla white or lilac with or without white at the base, campanulate, cup-shaped, or rotate, soon deciduous, glabrous or nearly so, about $1-2 \times$ as long as the calyx; tube shorter than the calyx; limb zygomorphic to actinomorphic, spreading; lobes 5 , imbricate in the bud, entire, more or less emarginate at the apex. Stamens included or exserted, five, equal, or four, often didynamous and mostly with a staminode; filaments glabrous, not contorted, free from each other, inserted on the base of the corolla; anthers free, oblong, orbicular, or reniform; cells often divergent at the base, confluent at the apex, dehiscent throughout by a longitudinal split, parallel and hardly confluent in the bud. Staminode glabrous, with a sterile anther. Ovary superior, glabrous, ovoid or globose; style mostly curved; stigma saucer-shaped or obscurely bilobed. Disk none. Placentae bilamellate, ovuliferous on both surfaces. Capsule included in the calyx, apiculate by the persisting style, 2- or 4-valved, loculicidal, and when 4-valved also septicidal; valves somewhat translucent, deciduous. Seeds brown, shining, ellipsoid, obliquely or sometimes longitudinally striate or reticulate-striate, with endosperm. Embryo straight; cotyledons short.

Distribution: 12 described species in tropical America.

Key to the species:

1. Calyx not angled, not inflated . . . . . . . . Calyx angled, seemingly inflated. Brazil (Paraná, Santa Gatarina) . ........ . . . N. reitzii

2. Leaves green beneath; inflorescence several-flowered, with bracts . . . . . . . . . . . . .

Leaves purple beneath; inflorescence usually 1 -flowered and without bracts; small plants, about $5-7 \mathrm{~cm}$ high. CoLOMBIA . ........... . . . $\mathbf{N}$. riparius

3. Leaves acuminate or acute, glabrous and glaucous above; inflorescence about half as long as the leaves, manyflowered; corolla campanulate; stamens 4, didynamous, included. Bolrvis . . . . . 11. N. rigidus

Leaves acute or obtuse, usually hairy and dark green above; inflorescence shorter or longer than the leaves; corolla campanulate or not ............

4. Leaves glabrous above; inflorescence racemose-paniculate, many-flowered; flowers small; flowering calyx 4-5 mm, fruiting one up to $7 \mathrm{~mm}$ long; corolla rotate; stamens 4, subequal, exserted. Costa Riga, Panama (see also $\mathcal{N}$. ecuadoriensis) . . . . . 2. N. apodemus

Leaves - at least some - hairy above; the younger ones mostly hairy; inflorescence usually not paniculate; flowers larger or not; corolla rotate or not . . . . . . . . 
5. Sepals connate for one-third to one-half of their length; calyx with mature fruit sometimes partially splitting; bracts often shorter than $10 \mathrm{~mm}$ (see also 2. $\mathcal{N}$. apodemus)

Sepals connate at the base up to one-fourth of their length; mostly many large many-flowered inflorescences which are usually longer than the leaves; bracts rather large, mostly longer than $10 \mathrm{~mm}$.............

6. Sepals $2-3 \times$ as long as wide; corolla $1 \frac{1}{2}-2 \times$ as long as the calyx; stamens 4 or 5 , equal, exserted; leaves dentate towards the apex, mostly about $3 \times$ as long as wide. Trinidad, Tobago ..... 12. N. subacaulis

Sepals $3-5 \times$ as long as wide; leaves when dentate towards the apex $4 \times$ as long as wide or more .......

7. Sepals connate up to one-third of their length, about $3 \times$ as long as wide, mostly about $5-6 \mathrm{~mm}$ long; leaves mostly acute, subentire or remotely serrate-dentate; stamens 4 or 5; seeds $0.2-0.3 \mathrm{~mm}$ long, not spinose (also in $\mathcal{N}$. ecuadoriensis ?) . . . . . . . . . . . .

Sepals connate for one-third to one-half of their length, 3-5 $\times$ as long as wide, mostly more than $8 \mathrm{~mm}$ long, leaves subentire to crenate-serrate; stamens 4 ; seeds $0.5 \mathrm{~mm}$ long

8. Seeds spirally striate, spinose; leaves beneath with many stomata-groups, but less than in the following species. Brazil (Rio de Janeiro, São Paulo) . . . . . . . . . . 6. N. primulifolius

Seeds reticulate-striate, not spinose; leaves beneath densely covered with stomata-groups .......6a. N. sp.

9. Leaves mostly - at least the younger ones - sparsely pilose above ................. . . .

Leaves practically. glabrous above, also the younger ones. Ecuador, Colombia (?) . . . 4. N. ecuadoriensis

10. Bracts $2-4 \mathrm{~mm}$ long; flowering calyx $3 \mathrm{~mm}$ (or more ?), fruiting one up to $6 \mathrm{~mm}$ long; stamens 4 (or 5?, no complete corolla known). FreNCH GuIANA . . 5. N. jelskii

Bracts 3-15 mm long; flowering calyx 4.5-6 mm, fruiting one up to $9 \mathrm{~mm}$ long; corolla campanulate, $6-10 \mathrm{~mm}$ long; stamens 4, didynamous, included. BoLIvia 1. N. andinus

11. (5). Lower bracts large, about $2 \times$ as long as wide, 13-20 $\mathrm{mm}$ long; plant large; leaves strigillose above with very delicate hairs; inflorescence few-flowered, about as long as the leaves; corolla campanulate; stamens 4, didynamous, included. Guatemala ... 3. N. bracteatus

Lower bracts 3-5 $\times$ as long as wide; inflorescence mostly many-flowered, mostly longer than the leaves . . . . .

12. Leaves dentate towards the apex; corolla cup-shaped, actinomorphic; stamens 5, equal, hardly included. VENEzUELA . . . . . . . 7. $\mathbf{N}$. primulinus

Leaves subentire; corolla and stamens unknown. ECUADOR 
1. N. andinus Rusby, Mem. Torr. Bot. Cl. 6: 98.1896 [J. D. Smith, Bot. Gaz. 20: 6. 1895, nomen]; Fritsch, Sitz. Akad. Wiss. Wien 134, Abt. 1: 124. 1925; Solereder, Beih. Bot. Centralbl. 24 (2): 435.1909.

Type: Bolrvia: La Paz, between Tipuani and Guanai, Bang 1729 (NY, holotype, photographs: U, WAG, isotypes: BM, G, GH, K, LE, M, MO, PH, US, 2 sheets, W).

Leaves dark green above, paler beneath, oblong-spathulate, $2 \frac{1}{2}-5$ (mostly 4) $\times$ as long as wide, acute or obtuse at the apex, sinuate or remotely serrate, pilose (with partially branched hairs) on both sides, especially on the midrib and veins beneath. Inflorescence about as long as the leaves or shorter, subcymose. Bracts 3-15 $\times 1-5$ $\mathrm{mm}$. Calyx green; sepals connate to one-fourth or one-third of their length, lanceolate, $3-4 \times$ as long as wide, in flower $4.5-6 \times 1.5 \mathrm{~mm}$, in fruit up to $9 \times 2 \mathrm{~mm}$. Corolla white, somewhat longer than the calyx, broadly campanulate, 6-10 $\mathrm{mm}$ long, slightly ventricose; tube somewhat shorter than the calyx, 4-5 $\mathrm{mm}$ long; lobes subequal, oval. Stamens 4, didynamous, included. Seeds (immature) about $2 \times$ as long as wide, $0.2 \times 0.1 \mathrm{~mm}$, obliquely striate.

Plant 5-20 cm high. Stems short, stout, $0.5-13 \mathrm{~cm}$ long. Leaves sessile, oblique, 3-20 $\times 0.7-5 \mathrm{~cm}$, papyraceous when dry; primary veins 3-10 pairs. Inflorescence: peduncle slender, pilose, $0.5-10.5 \mathrm{~cm}$ long, bearing 2-8 branches, with some bracts. Bracts lanceolate, $3-5 \times$ as long as wide, acute, entire or remotely serrulate, pilose on both sides. Pedicels slender, pilose, 3-45 mm long. Sepals pilose outside, glabrous inside. Corolla glabrous on both sides; lobes $2.5-5 \times 1.5-4 \mathrm{~mm}$. Filaments: two ventral, $1.5-2 \mathrm{~mm}$, and two lateral, $2-2.5 \mathrm{~mm}$ long; anthers reniform, $0.7-1$ $\mathrm{mm}$ in diam.; cells divergent at the base. Staminode slender, $1.2 \mathrm{~mm}$ long, glabrous; sterile anther small. Ovary ovoid, $1.5 \times 1-1.2 \mathrm{~mm}$; style curved, glabrous, $3 \mathrm{~mm}$ long; stigma obscurely stomatomorphic. Capsule about half as long as the calyx, ovoid, $4 \times 2 \mathrm{~mm}$, bivalved. Seeds dark brown.

Distribution: Bolivia.

Ecology: On very moist floor in dense shady forests (teste Buchtien). Bolivia: LA PAz: San Carlos, near Mapiri, alt. $550 \mathrm{~m}$, Buchtien 1337 (US); ibid., alt. $700 \mathrm{~m}$, Buchtien 1921 (US); between Tipuani and Guania, Bang 1729 (NY, holotype, isotypes: BM, G, GH, K, LE, M, MO, PH, US, 2 sheets, W).

2. N. apodemus Donn. Sm., Bot. Gaz. 20: 6. 1895; Pittier, Prim. Fl. Costar., Anales Inst. Fisico-Geogr. Nac. 8: 199. 1898; Morton, Field Mus. Publ. Bot. 18: 1185. 1938; Contrib. U. S. Nat. Herb. 29: 40. 1944.

Fig. 4, p. 345

Type: Costa Rica: Llanos de Santa Clara, Jiménez, Comarca de Limón, J. D. Smith 5109 (US, holotype, photographs: U, WAG, isotype: $\mathrm{GH}$ ).

Leaves medium green above, paler beneath, oblong-spathulate, 2-5 (mostly 3-4) $\times$ as long as wide, acute or obtuse at the apex, entire or dentate towards the apex, practically glabrous (with scattered minute hairs) above, minutely puberulous beneath. Inflorescence shorter or longer than the leaves, racemose-paniculate. Bracts 3-7 $\times$ 0.5-1.5 mm. Calyx green or sometimes reddish; sepals connate at the base to about one-third of their length, lanceolate, $3-4 \times$ as long as wide, in flower 4-5 $\times 1-1.5 \mathrm{~mm}$, in fruit up to $7 \times 2.5 \mathrm{~mm}$. Corolla white, somewhat longer than the calyx, rotate, 5-6 $\mathrm{mm}$ long; 
A

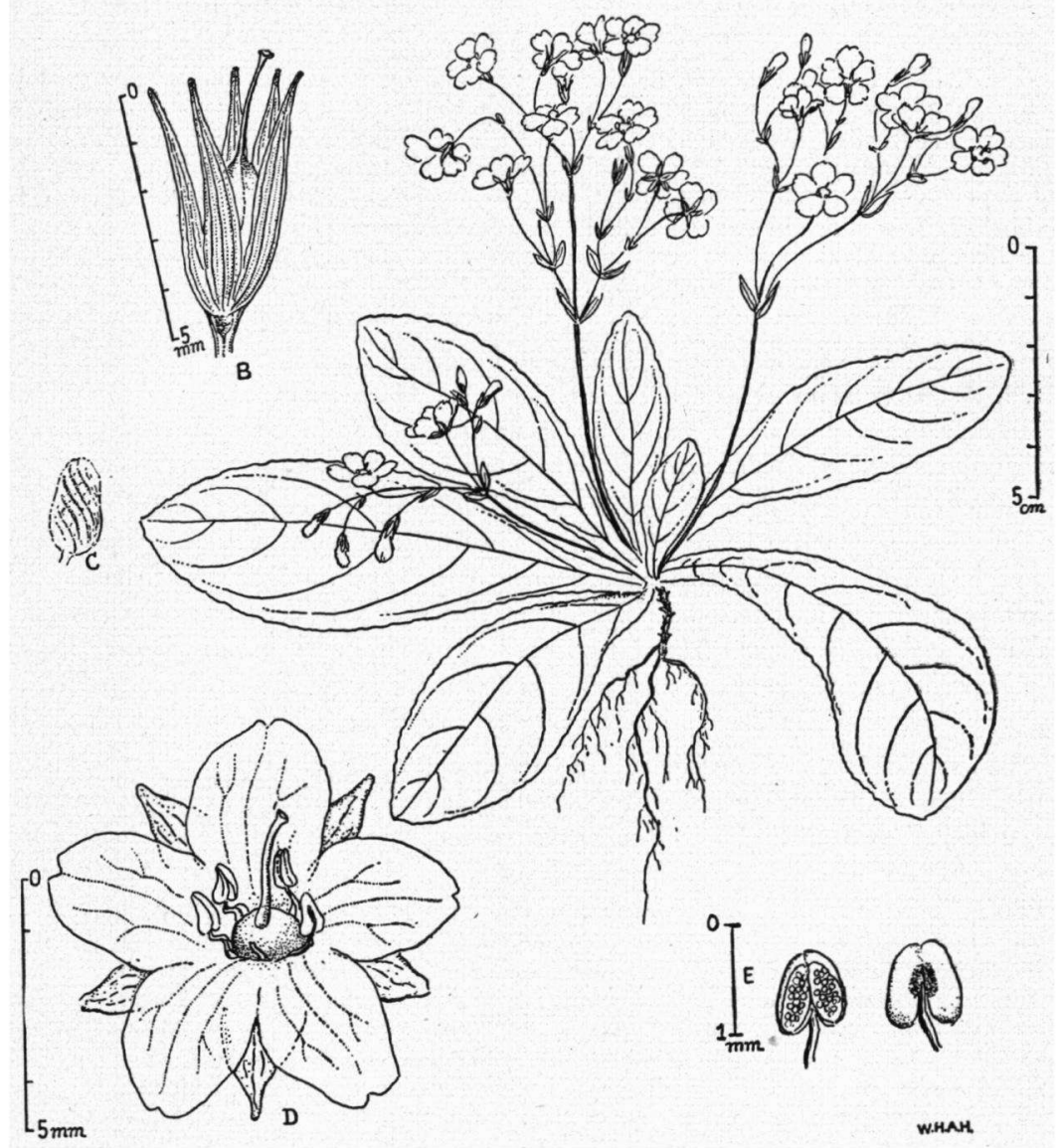

Fig. 4. Napeanthus apodemus (Skutch 4854): A. plant; B. fruit; C. seed; D. flower; E. anther, both sides.

tube very short, about $1.5 \mathrm{~mm}$ long; limb subactinomorphic; lobes oval. Stamens 4, equal or subequal (?), exserted. Seeds $1 \frac{1}{2}-2 \times$ as long as wide, $0.3 \times 0.15-0.2 \mathrm{~mm}$, obliquely striate.

Plant 5-15 cm high. Stems very short, $0.3-2 \mathrm{~cm}$ long. Leaves sessile, $2-14 \times 0.7-5$ cm or smaller, membranaceous when dry; primary veins $2-6$ pairs. Inflorescence: peduncle slender, terete, glabrous, 1-6 cm long, dichotomously branched, with small opposite bracts. Bracts linear-lanceolate, about $5 \times$ as long as wide, acuminate, entire, glabrous. Pedicels slender, glabrous, 6-25 mm long. Sepals sparsely pilose outside, glabrous inside. Corolla glabrous on both sides; lobes about $4 \times 2 \mathrm{~mm}$. Filaments about $1.5 \mathrm{~mm}$ long, widened at the base; anthers orbicular, $0.7-0.8 \mathrm{~mm}$ in diam.; cells divergent at the base. Staminode about half as long as the stamens, $1 \mathrm{~mm}$ long. Ovary ovoid, $1 \times 0.7 \mathrm{~mm}$; style glabrous, curved, $2 \mathrm{~mm}$ long; stigma saucer-shaped. Capsule about half as long as the calyx, globose. Seeds dark brown, mucronate at the base. 
Distribution: Costa Rica, Panama.

Ecology: In rainforests, from the coast to the mountains; alt. 0-900 m.

Costa Rica: La Colombina Farm, prov. Limón, Standley 36891 (US), 36918 (US); R. Verde, Santa Clara, J. D. Smith 6707 (US, K); La Concepción, Santa Clara, J. D. Smith 6709 (US, BM, GH, K, M, MO, NY, UC); Jiménez, Santa Clara, J. D. Smith 6708 (US); ibid., Comarca de Limón, J. D. Smith 5109 (US, holotype, isotype: GH); between Simón and Moin, Pittier 16008 (BM, GH); Isaki, Salamanca, Tonduz 9553 (BM); Los Ayotes, near Tilarán, prov. Guanacaste, Standley \& Valerio 45390 (US); Basin of El General, prov. San José, Skutch 4854 (MO, US); Siquirres, Kupper 454 (M).

Panama: Darien: between Cana and Cuasi, Chepigana, Terry \& Terry 1471 (GH, MO).

3. N. bracteatus Morton, Contrib. U.S. Nat. Herb. 29: 39. 1944. Type: Guatemala: Alta Verapaz, near Finca Sepacuité, Cook \& Griggs 614 (US, holotype, photographs: U, WAG).

Leaves dark green above, paler beneath, oblanceolate, $3 \frac{1}{2}-4 \times$ as long as wide, acute at the apex, denticulate, strigillose above with very delicate tortuous hairs, subtomentose beneath, especially on the midrib and veins. Inflorescences numerous, subumbellate or subcymose. Lower bracts large, 13-20 × 7-9 mm. Calyx green; sepals connate at the base, lanceolate, about $4 \times$ as long as wide, in flower about $9 \times 2.5 \mathrm{~mm}$, in fruit up to $10 \times 2.5 \mathrm{~mm}$ (or more?). Corolla white, campanulate, about $12 \mathrm{~mm}$ long; tube about $6 \mathrm{~mm}$ long; limb slightly bilabiate; lobes suborbicular. Stamens 4, didynamous, included. Seeds $1 \frac{1}{2}-2 \frac{1}{2} \times$ as long as wide, $0.5 \times 0.2-0.3 \mathrm{~mm}$, obliquely striate.

Plant about $25 \mathrm{~cm}$ high. Stems short, 5-10 cm long.' Leaves 8-27 $\times 2-8 \mathrm{~cm}$, membranaceous when dry, subcordate at the extreme base; primary veins $10-15$ pairs, arcuate and anastomosing towards the margin. Inflorescence $10-14 \mathrm{~cm}$ long. Peduncle sparsely pilose, 5-10 cm long, bibracteate at the apex. Bracts ovate or ovate-lanceolate, $1 \frac{1}{2}-3 \times$ as long as wide, acute, entire, sparsely pilose on both sides, the lower ones largest. Pedicels slender, practically glabrous, $2-5 \mathrm{~cm}$ long. Sepals sparsely pilose outside, glabrous inside, ciliate. Corolla very minutely puberulous outside, glabrous inside; lobes 6-7 mm long, not ciliate. Filaments: two ventral, $3 \mathrm{~mm}$, and two lateral, $2 \mathrm{~mm}$ long; anthers oblong, $1-1.5 \mathrm{~mm}$ long. Staminode none (fide Morton). Ovary ovoid, $3 \times 1.5 \mathrm{~mm}$; style glabrous, $5 \mathrm{~mm}$ long; stigma saucer-shaped. Capsule about half as long as the calyx (?), $5 \times 2.5$ (?) $\mathrm{mm}$. Seeds dark brown, mucronate at the base.

Distribution: Guatemala.

Ecology: In dense montane rainforests; alt. 1500-1600 m (teste Standley).

Guatemala: Alta Verapaz: near Finca Sepacuité, Cook \& Griggs 614 (US, holotype); between Tactic and the divide on road to Tamahú, Standley 90621 a (US, paratype), 90623 (US, paratype); east of Tactic, near Tamahú, Standley 71201 (US, paratype).

4. N. ecuadoriensis Fritsch, Sitzungsb. Hofm. Wien 134, Abt. 1: 125. 1925.

Type: Ecuador: along Peripa R., Sodiro 119/57, not seen, destroyed in Berlin.

Leaves paler beneath, large, oblong-spathulate, dentate, nearly glabrous above, puberulous beneath, especially on the midrib and 
veins. Inflorescence shorter than the leaves; few-flowered. Sepals highly connate, oblong, in fruit $5 \mathrm{~mm}$ long. Corolla and stamens unknown.

Plant with the habit of $\mathcal{N}$. primulinus. Stems over $10 \mathrm{~cm}$ long. Leaves subsessile (with a winged petiole), 8-16 × 3-5 cm; primary veins 8-10 pairs. Inflorescence 4-6 cm long. Peduncle slender, hirtellous. Bracts narrow, pilose beneath. Sepals pilosulous outside. Fruit shorter than the calyx, nearly $5 \mathrm{~mm}$ long.

No specimens seen. Description compiled.

The following specimens may belong to this species:

Colombia: Valle del Cauca: R. Digua, La Elsa, Quebrada La Cristalina, Cuatrecasas 15237 (F, US); R. Digua Valley, along R. Engaña, Killip 34853 (US, BM).

5. N. jelskii Fritsch, Sitzungsb. Akad. Wiss. Wien 134, Abt. 1: 124. 1925.

Type: French Guiana: Cayenne, Jelski s.n., not seen, destroyed in Berlin.

Leaves oblong-spathulate, $2-4 \times$ as long as wide, $1.5-14 \times 0.8-4.5$ $\mathrm{cm}$, obtuse or rounded at the apex, remotely serrate, sinuate, or subentire. Peduncle filiform. Bracts small, 2-4 × 0.4-1 mm. Sepals connate at the base to one-third of their length, lanceolate, about $3 \times$ as long as wide, in flower $3 \times 1 \mathrm{~mm}$ (or more?), in fruit up to $6 \times 2 \mathrm{~mm}$. Corolla (only seen in bud) pilose outside; limb subactinomorphic. Stamens four with a staminode. Seeds about $2 \times$ as long as wide, about $0.3 \times 0.15 \mathrm{~mm}$.

Plant 5-10 cm high. Stems very short, 0.5-2 cm long. Leaves medium green above, paler beneath, membranaceous when dry, sparsely and minutely pilose on both sides, especially beneath. Inflorescence cymose. Peduncle sparsely pilose, 2.5-6 cm long. Bracts lanceolate, about $4 \times$ as long as wide, acuminate, entire, pilose on both sides. Pedicels filiform, sparsely pilose, $10-25 \mathrm{~mm}$ long. Sepals pilose outside, glabrous inside. Corolla white (?), glabrous inside; lobes rounded. Filaments (in the bud) of the stamens and of the staminode equal, $0.5 \mathrm{~mm}$ long. Ovary globose, $1 \mathrm{~mm}$ in diam.; style glabrous; stigma obscurely saucer-shaped. Capsule one-third of the length of the calyx, globose, $1.5 \mathrm{~mm}$ in diam., bivalved. Seeds dark brown.

Distribution: French Guiana.

Ecology: no data extant.

French GuIANA: Acarouany R., Lower Mana R., Mélinon 58 (P); Leprieur, anno $1838(\mathrm{P})$.

The following specimens may belong to a new species closely related to $\mathcal{N}$. jelskii, but the material is insufficient for description. They differ by the leaves which are 4-12 $\times$ as long as wide, $4-24 \times 0.8-3.5 \mathrm{~cm}$, acute or acuminate at the apex, and more hairy above. Stamens 5 (or 4 with a staminode ?).

French Guiana: Cayenne, anonym. (G, U); De Candolle \& l'Héritier s.n. (G); Poiteau s.n. (K, P, U); L. C. Kichard s.n. (P).

Guiana: Frfnch?: Boordes (?) s.n. (C).

6. N. primulifolius (Raddi) Sandwith, Webbia 22 (1): 332. 1956.

Basionym: Oxalis primulaefolia Raddi, Memoria in, Atti Soc. Ital. Sci. Modena 18: 400. 1820.

Type: BrazIL: Rio de Janeiro, near Mandiocca, Raddi s.n. (holotype and isotype: PI, not seen, photographs of both sheets: $\mathrm{K}, \mathrm{U}$ ). 
Heterotypic synonym: Napeanthus brasiliensis Gardn., Lond. Journ. Bot. 2: 13. 1843; D.C., Prod. 9: 275. 1845; J. D. Smith, Bot. Gaz. 20: 6. 1895; Fritsch in Engl., Bot. Jahrb. 37: 482. 1906; Sitzungsb. Akad. Wiss. Wien 134, Abt. 1: 122. 1925; Solereder, Beih. Bot. Centralbl. 24 (2) : 432. 1909; Glaziou, Bull. Soc. Bot. France, Mem. 3: 515. 1911. Type: Brazil: Rio de Janeiro, Serra das Orgãos, Gardner 581 ( $\mathrm{K}$, holotype, isotypes: BM, CGE, FI, G, 2 sheets, $\mathrm{K}, \mathrm{NY}, 2$ sheets, $\mathrm{P}, \mathrm{W}$, photographs: of one G sheet in F and US; of one NY sheet in F and US, of P sheet in U and WAG).

Leaves dark green above, paler beneath, oblong or oblong-spathulate, usually 3-4 (rarely up to 5 ) $\times$ as long as wide, acute, obtuse, or rounded at the apex, crenate-serrate or subentire, practically glabrous above, puberulous beneath. Inflorescence shorter to longer than the leaves, subumbellate or subcymose. Bracts 3-12 $\times 1-4 \mathrm{~mm}$. Calyx green; sepals connate for one-third to one-half of their length, oblong or lanceolate, $3-5 \times$ as long as wide, in flower $5-9 \times 1-3 \mathrm{~mm}$, in fruit up to $13 \times 5 \mathrm{~mm}$. Corolla white, campanulate, $13-14 \mathrm{~mm}$ long, dorsally somewhat ventricose; tube somewhat shorter than the calyx, about $7 \mathrm{~mm}$ long; limb rather zygomorphic; lobes obovate. Stamens 4 , didynamous, included. Seeds $1 \frac{1}{2}-2 \times$ as long as wide, $0.5 \times 0.25-0.3 \mathrm{~mm}$, spirally striate, spinose.

Plant 10-25 cm high. Stems short, 3-15 cm long. Leaves subsessile, 5-23 $\times 1.5-6.5$ cm or sometimes smaller, papyraceous when dry, subcordate at the extreme base; primary veins 6-9 pairs. Inflorescence 4-24 cm long. Peduncle slender, practically glabrous, 1.2-14 cm long. Bracts oblong or lanceolate, 3-5 (rarely 2) $\times$ as long as wide, acute or acuminate, puberulous on both sides or only beneath. Flowers 1-many. Pedicels slender, practically glabrous, $1.5-6 \mathrm{~cm}$ long. Sepals more or less pilose outside, glabrous inside. Corolla glabrous on both sides; lobes 6-7 $74-6 \mathrm{~mm}$. Filaments: two ventral, $3.5 \mathrm{~mm}$, two lateral, $3 \mathrm{~mm}$ long; anthers oblong-reniform, $0.75 \mathrm{~mm}$ long. Staminode $0.8 \mathrm{~mm}$ long. Ovary ovoid, $2 \times 1.5 \mathrm{~mm}$; style erect, glabrous, 4-5 mm long; stigma obscurely bilobed. Capsule ovoid, $\frac{1}{2}-1 \times$ as long as the calyx, 4-6 $\times 2-3 \mathrm{~mm}$, apiculate, bivalved. Seeds medium brown.

Distribution: Brazil (Rio de Janeiro, São Paulo).

Ecology: On banks of watercourses, in montane rainforests.

Brazil: Rio de Janeiro: Serra das Orgãos, Gardner 581 (K, holotype of $\mathcal{N}$. brasiliensis; isotypes: BM, CGE, G, 2 sheets, $K, N Y, 2$ sheets, $P, W$ ); ibid., herb. Miers 4231 (BM); ibid., Ule 2417 (HBG); ibid., herb. Schwacke 4394 (GOET); near Mandiocca, St. Hilaire, annis 1816-1821 (P); ibid., Raddi s.n. (PI, 2 sheets, type, not seen, photographs: K, U); Palmeiras, Glaziou 8835 (P, G, K); Alto Macahé, Glaziou $18142(\mathrm{P}, \mathrm{K}), 19745(\mathrm{P}, \mathrm{C}, \mathrm{LE})$; Langsdorff, anno $1821(\mathrm{P})$; Glaziou 1075 (P, BR).

Rio DE JANEIRo ?: Bowie \& Cunningham 19 (BM).

SȦo Paulo: Ubatuba, Guillemin 620 (P).

6a. N. sp.

Several specimens have been collected in Guiana which resemble $\mathcal{N}$. primulifolius, but they differ in the following characters: seeds reticulate-striate, not spinose. Leaves beneath with numerous stomata groups, more than the specimens from southern Brazil. No complete corolla seen. In bud 4 stamens and one staminode. They probably belong to a new species. 
Suriname: Emmaketen, Stahel 183 (U); Nassau Mts., Lanjouw \& Lindeman $2630(U)$.

French Guiana: Leprieur, anno 1840 (P), s.n. (G); L. C. Richard s.n. (P); herb. expos. col. s.n. (P).

7. N. primulinus (Karst.) Benth. et Hook. ex Jackson, Ind. Kew. 2: 292. 1894 (see also Benth. \& Hook., Gen. Pl. 2: 1018. 1876); J. D. Smith, Bot. Gaz. 20: 6. 1895; Knuth, Init. Fl. Venez. in Fedde, Repert. Beih. 43: 642. 1928.

Basionym: Marssonia primulina Karst., Fl. Colomb. 1: 97, t. 48. 1860; Urban, Symb. Ant. 2: 347. 1901 (exclusive of first and second synonym); Solereder, Beih. Bot. Centralbl. 24 (2): 433. 1909.

Type: Venezuela: Mérida, Tovar, Karsten s.n. (LE, holotype, isotypes: LE, 2 sheets, $W, 3$ sheets, photographs of one $W$ sheet in $\mathrm{U}$ and $\mathrm{WAG}$ ).

Leaves dull dark green above, paler beneath, oblong-spathulate or oblanceolate, 3-6 $\times$ as long as wide, acute or acuminate, dentate towards the apex, practically glabrous above, subvillose beneath, especially on the midrib, the veins, and the base; hairs white or purplish.. Inflorescence mostly longer than the leaves, paniculate. Bracts 10-25 × 3-7 mm. Calyx green; sepals connate at the base, oblong or lanceolate, $2-3 \frac{1}{2} \times$ as long as wide, in flower 5-7 $\times 2 \mathrm{~mm}$, in fruit up to $8 \times 3 \mathrm{~mm}$. Corolla white or lilac with white at the base (teste Steyermark), about $2 \times$ as long as the calyx, cup-shaped, 13-15 mm long; tube short, about $4 \mathrm{~mm}$ long; limb actinomorphic; lobes imbricate in the bud, not contorted, obovate. Stamens 5, equal, hardly included. Seeds $1 \frac{1}{2}-2 \times$ as long as wide, $0.3-0.4 \times 0.2-0.25$ $\mathrm{mm}$, obliquely striate, obscurely reticulate.

Plant 5-45 cm high. Stems short, 3-15 cm long. Leaves subsessile, 6.5-28 $\times 2.5-7$ $\mathrm{cm}$, papyraceous or chartaceous when dry, subcordate at the extreme base; primary veins 5-8 pairs. Inflorescence up to $45 \mathrm{~cm}$ long, proliferous in the axils of the bracts. Peduncle slender, sparsely pilose to glabrous, $3-21 \mathrm{~cm}$ long. Bracts opposite, lanceolate, 3-5 $\times$ as long as wide, acuminate, obscurely dentate, pilose on both sides, especially beneath. Pedicels slender, sparsely pilose to glabrous, 1-5 cm long. Flowers 5-, rarely 6-merous. Sepals sparsely pilose outside, glabrous inside. Corolla glabrous on both sides; lobes 9-11 $\times$ 5-7 mm. Filaments $3 \mathrm{~mm}$ long, widened at the base; anthers reniform, $1 \times 1.5 \mathrm{~mm}$; cells divergent at the base. Ovary ovoid, $2.5 \times 1.7 \mathrm{~mm}$; style slightly curved, glabrous, $4 \mathrm{~mm}$ long; stigma obscurely bilobed. Capsule ovoid or subglobose, $3 \times 2.5 \mathrm{~mm}$, apiculate, bivalved. Seeds medium brown.

Distribution: Venezuela.

Ecology: On shaded moist slopes (teste Steyermark); alt. about $2000 \mathrm{~m}$.

Venezuela: MERIDA: Tovar, Karsten s.n. (LE, holotype, isotypes: LE, 2 sheets, W, 3 sheets); ibid., Moritz 870 (BM).

Merida ?: near Tovar, Fendler 770 (GH, GOET, K, MO, NY, PH).

D.F.: east of El Junquito, Steyermark 57016 (F, K, NY, S, US).

8. N. reitzii (L. B. Smith) Burtt ined.?

Fig. 5, p. 350

Basionym: Hatschbachia reitzii L. B. Smith, Anais Bot. Herb. Barb. Rodr. 5 (5): 37, with fig. 1953. 


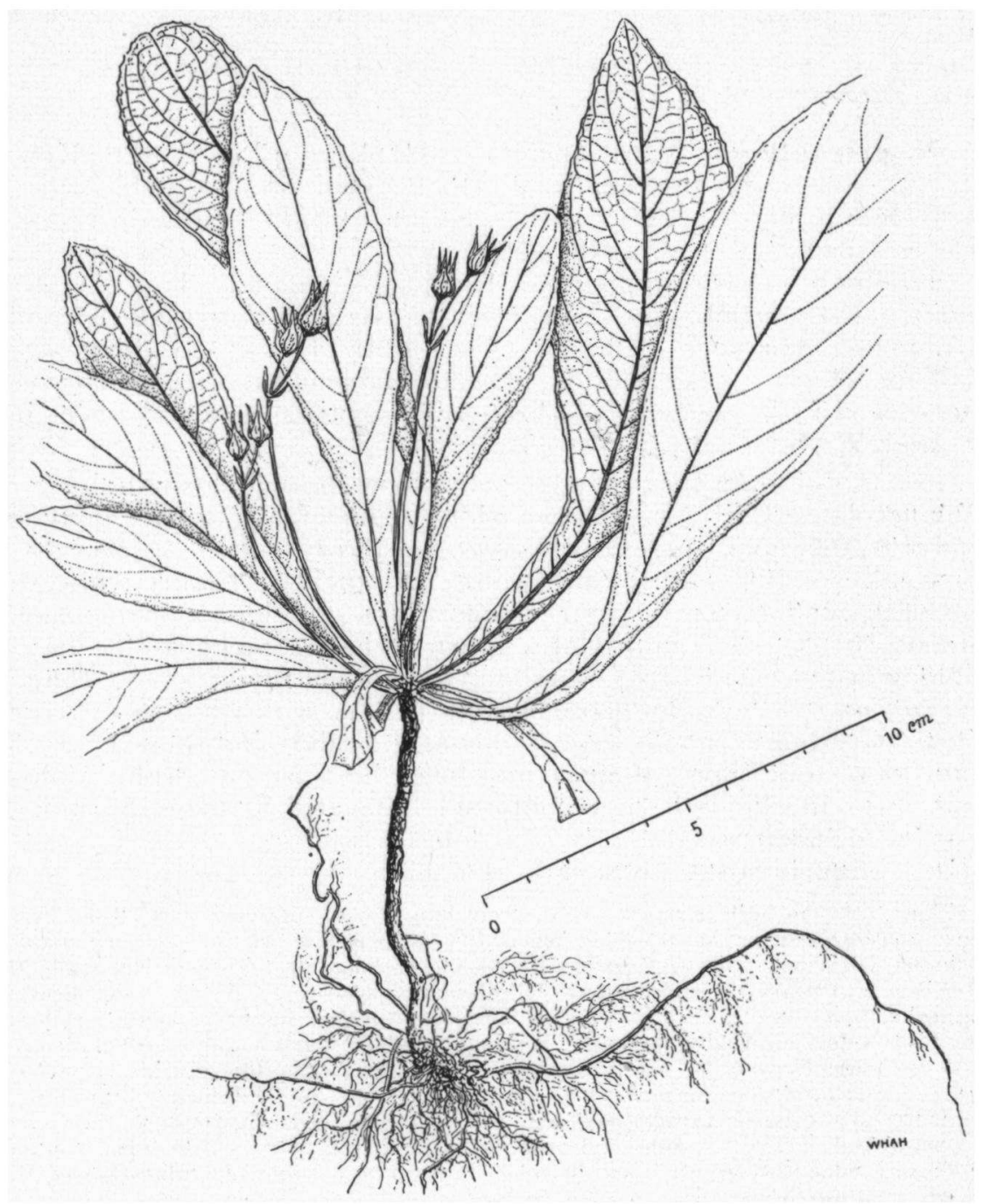

Fig. 5. Napeanthus reitzii (Dusén 3693).

Type: Brazil: Paraná, Munic. de Guaratuba (São José dos Pinhaes), Garuva, Hatschbach 1717 (US, holotype, not seen, photographs: $\mathbf{K}, \mathbf{U})$.

Leaves medium green above, paler beneath, oblong-spathulate or oblanceolate, $4-5 \frac{1}{2} \times$ as long as wide, acute at the apex, subentire or remotely serrate towards the apex, glabrous above or only at the base sparsely pubescent, appressed-pubescent beneath. Inflorescence few-flowered, shorter than the leaves. Bracts 5-9 $\times 1,3 \mathrm{~mm}$. Calyx green, angled, entirely closed after anthesis and therefore seemingly' 
inflated; sepals connate to about one-third of their length, oblongtriangular, $1 \frac{1}{2}-2 \times$ as long as wide, $10-12 \times 5-6 \mathrm{~mm}$, tapering from the base to the apex, in fruit up to $14 \times 8 \mathrm{~mm}$ (or more ?): Corolla lilac with white at the base (teste Hatschbach), somewhat longer than the calyx, campanulate, $15 \mathrm{~mm}$ long; lobes subequal (?). Stamens 4 , didynamous (?), included. Seeds about $0.4 \mathrm{~mm}$ long, longitudinally or obliquely striate.

Plant 15-30 cm high. Stems short, 10-12 cm long. Leaves opposite, oblique, 5-21 $\times 1-4.8 \mathrm{~cm}$, papyraceous when dry; primary veins 4-8 pairs. Inflorescence: peduncle sparsely pubescent, $6-9 \mathrm{~mm}$ long, with two bracts at the apex. Bracts oblong-lanceolate, $3-5 \times$ as long as wide, acuminate, entire, glabrous above, sparsely pubescent beneath. Pedicels short, sparsely pubescent, 6-18 mm long, thickened towards the calyx. Sepals sparsely pubescent outside, especially at the base, glabrous inside. Corolla glabrous on both sides. Filaments widened at the base; anthers oblong-elliptic, $1.2 \mathrm{~mm}$ long. Ovary ovoid; style with scattered hairs, $6 \mathrm{~mm}$ long; stigma saucer-shaped. Placentae 2, parietal, bilamellate, ovuliferous on both surfaces. Fruit: only immature capsule seen. Seeds dark brown.

Description of corolla and stamens compiled. Corolla only seen on photograph of type.

Distribution: Brazil (Paraná, Santa Catarina).

Ecology: On moist slopes, in dense rainforests (teste Hatschbach). Brazil: Paraná: Munic. de Guaratuba, Garuva, Hatschbach 1717 (US, holotype, not seen, photographs: K, U); Volta Grande, Marumby, alt. about $600 \mathrm{~m}$, Dusén 3693 (S).

Santa Catarina: Joinville, Reitz 3. 811 (HBR, paratype, not seen).

9. N. rigidus Rusby, Mem. Torr. Bot. Cl. 6: 99. 1896; Solereder, Beih. Bot. Centralbl. 24 (2): 435. 1909; Fritsch, Sitzungsb. Akad. Wiss. Wien 134, Abt. 1: 122. 1925.

Fig. 6, p. 352

Type: Bolrvia: La Paz, between Tipuani and Guanai, Bang 1729a (NY, holotype, photographs: $\mathrm{U}, \mathrm{WAG}$, isotypes: $\mathrm{MO}, \mathrm{PH}$ ).

Leaves glabrous and glaucous above, subtomentose beneath, lanceolate, $3-5 \times$ as long as wide, acute or acuminate at the apex, coarsely short-serrate, the teeth mostly sharp, thick, and rigid. Inflorescence about half as long as the leaves, umbellate. Bracts 5-11 $\times$ 1-2 mm. Calyx green; sepals connate to about one-third of their

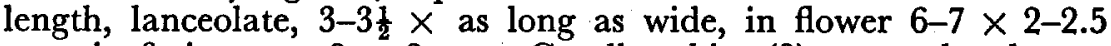
$\mathrm{mm}$, in fruit up to $9 \times 3 \mathrm{~mm}$. Corolla white (?), somewhat longer than the calyx, campanulate, $9 \mathrm{~mm}$ long, slightly ventricose; tube somewhat shorter than the calyx, 5-6 mm long; limb oblique; lobes subequal, ovate. Stamens 4 , slightly unequal, included. Seeds about $2 \times$ as long as wide, $0.4 \times 0.2 \mathrm{~mm}$, spirally striate.

Plant $10-20 \mathrm{~cm}$ high. Stems short, stout, appressed-pubescent at the apex, glabrescent, 5-10 cm long. Leaves opposite, sessile, 7-12 $\times 1.5-3 \mathrm{~cm}$, papyraceous when dry; primary veins 4-7 pairs. Inflorescence: peduncle short, pilose, 1-2 cm long. Bracts linear-lanceolate, about $5 \times$ as long as wide, acute, entire, glabrous above, pilose beneath. Pedicels rigid, erect, sparsely pilose, 1-2 cm long. Sepals pilose outside, glabrous inside. Corolla at the base and at the throat $3.5 \mathrm{~mm}$ wide, glabrous on both sides; lobes $3 \times 2-2.5 \mathrm{~mm}$, obscurely emarginate at the apex. Filaments slightly widened at the base, two ventral, $2.25 \mathrm{~mm}$, and two lateral, $2 \mathrm{~mm}$ long; anthers oblong, connivent (?), $0.75 \times 0.6 \mathrm{~mm}$; cells parallel. Staminode erect, $1.7 \mathrm{~mm}$ long. Ovary ovoid, $2 \times 1.5 \mathrm{~mm}$; style curved, glabrous, $4 \mathrm{~mm}$ long; stigma obscurely bilobed. Capsule bright green (fide Rusby), about half as long 
as the calyx, ovoid, $4 \times 3 \mathrm{~mm}$, acute at the apex, bivalved. Seeds dark brown, obliquely ellipsoid, mucronate at the base.

Distribution: Once collected in Bolivia.

Ecology: No data extant.

Bolrvia: LA PAz: between Tipuani and Guanai, Bang 1729a (NY, holotype, isotypes: $\mathrm{MO}, \mathrm{PH})$.

10. N. riparius Philipson, Mutisia 24: 6. 1956.

Type: Colombia: Meta, Sierra de la Macarena, R. Guapaya, Philipson, Idrobo \& Fernández 1679 (BM, holotype).

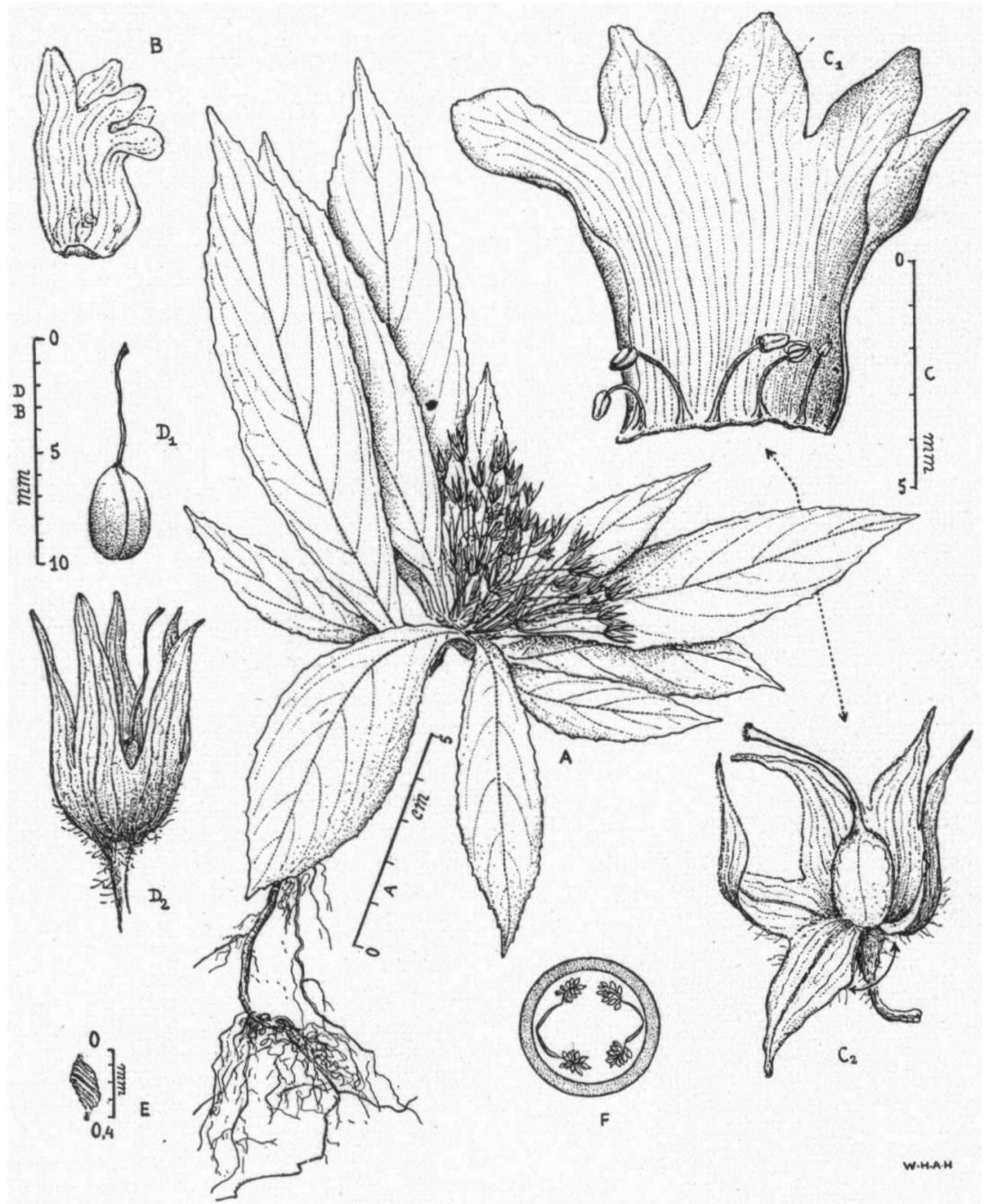

Fig. 6. Napeanthus rigidus (Bang 1729a): A. plant; B. corolla; $\mathrm{C}_{1}$. opened corolla; $C_{2}$. calyx with pistil; $D_{1}$. capsule; $D_{2}$. fruiting calyx; $E$. seed. 
Leaves dark green above, purple beneath, oblong-lanceolate, oblong, or oblanceolate, $2-4 \times$ as long as wide, obtuse or rounded at the apex, subentire or often obscurely serrate near the apex, pilose on both sides. Inflorescence shorter than the leaves, 1-3-flowered. Peduncle when present very short, about $3 \mathrm{~mm}$ long, with two bracts. Bracts 4-7 $\times 1.5-2 \mathrm{~mm}$. Calyx green; sepals connate to about onehalf of their length, oblong-lanceolate, $2 \frac{1}{2}-4 \times$ as long as wide, in flower 4-6 $\times 1-1.5 \mathrm{~mm}$, in fruit up to $9 \times 2.5 \mathrm{~mm}$. Corolla white (testibus Philipson, Idrobo \& Fernández) or pale lilac (teste Haught), somewhat longer than the calyx, campanulate, 6-9 $\mathrm{mm}$ long; tube 3-4.5 mm long; limb subactinomorphic, lobes nearly rectangular. Stamens 4, didynamous, included. Seeds $1 \frac{1}{2}-2 \times$ as long as wide, $0.25-0.3 \times 0.15-0.2 \mathrm{~mm}$, obliquely striate.

Plant about 5-7 cm high. Stems very short, $0.5-1.5 \mathrm{~cm}$ long. Leaves 3-8.5 $\times$ $0.9-4.3 \mathrm{~cm}$, papyraceous when dry, subcordate at the extreme base; primary veins 4-6 pairs. Inflorescence: bracts oblong-lanceolate, about $3 \times$ as long as wide, acuminate, subentire, sparsely pilose on both sides. Pedicels slender, sparingly pilose or practically glabrous, $2.5-4 \mathrm{~cm}$ long. Sepals sparsely pilose outside, glabrous inside. Corolla glabrous on both sides or nearly so, obscurely ciliolate; lobes 3-4.5 $\times 2-3 \mathrm{~mm}$. Filaments widened at the base, two ventral, about $1.5 \mathrm{~mm}$ long, two lateral, about $1 \mathrm{~mm}$ long; anthers oblong-reniform, $0.75 \times 0.5 \mathrm{~mm}$. Staminode half as long as the stamens, $1 \mathrm{~mm}$ long. Ovary ovoid, about $1.5 \times 1 \mathrm{~mm}$; style slightly curved, glabrous, $2 \mathrm{~mm}$ long; stigma obscurely bilobed. Capsule subglobose, about half as long as the calyx, $4 \times 3 \mathrm{~mm}$. Seeds dark brown.

Distribution: Colombia.

Ecology: On riverbanks, in rainforests; alt. about 500-600 m.

Colombra: Meta: Sierra de la Macarena, R. Guapaya, Philipson, Idrobo \& Fernández 1679 (BM, holotype); Sierra de la Macarena, Caño Ciervo, Philipson, Idrobo \& Jaramillo 2051 (BM, paratype); Villavicencio, Haught 2523 (US).

11. N. robustus Fritsch, Sitzungsb. Akad. Wiss. Wien 134, Abt. 1: 122. 1925.

Type: Ecuador: near S. Domingo, Sodiro 119/58, not seen, destroyed in Berlin.

Leaves paler beneath, large, oblong-spathulate, acute at the apex, subentire, nearly glabrous above, pubescent-tomentose beneath. Inflorescences many, elongate, longer than the leaves, loose-flowered. Bracts 7-15 $\times 2-5 \mathrm{~mm}$. Sepals connate at the base, oblong-lanceolate, in fruit 6-7 mm long. Corolla delicate; lobes rounded.

Stems thick, elongate, about $20 \mathrm{~cm}$ long. Leaves $17-24 \times 5.5-7.5 \mathrm{~cm}$; primary veins 6-8 pairs. Inflorescence up to $50 \mathrm{~cm}$ long. Peduncle pilose. Bracts lanceolate. Pedicels elongate, slender, curved, sparsely pilose, about $2 \mathrm{~cm}$ long. Sepals pilose outside. Style curved. Capsule shorter than the calyx, about $5 \mathrm{~mm}$ long.

No specimens seen. Description compiled.

12. N. subacaulis (Griseb.) Benth. et Hook. ex Kuntze, Rev. 2: 474. 1891 (see also Benth. \& Hook., Gen. Pl. 2: 1018. 1876); J. D. Smith, Bot. Gaz. 20: 6. 1895; Sandwith. Kew Bull. 1938: 371.

Basionym: Episcia (?) subacaulis Griseb., Fl. Br. W.I. Isl. 462. 1861 (“1864"); Solereder, Beih. Bot. Centralbl. 24 (2): 433. 1909.

Type: TRINIDAD: Las Cuevas, Crueger s.n. (GOET, holotype, isotype: $\mathrm{K}$, photographs: U, WAG). 
Homotypic synonym: Marssonia subacaulis (Griseb.) Urb., Symb. Ant. 7: 377. 1912; Morton in Fl. Trin. and Tob. 2 (5): 302.1955.

Leaves dark green above, paler beneath, oblong or oblong-spathulate, 2-4 (mostly 3) $\times$ as long as wide, acute or obtuse at the apex, dentate near the apex, sparsely strigillose to glabrous above, sparsely pubescent beneath. Inflorescence mostly shorter than the leaves, racemose, few-flowered. Bracts 3-10 $\times 0.5-3 \mathrm{~mm}$. Calyx green; sepals connate to about one-third of their length, oblong or lanceolate, $2-3 \times$ as long as wide, in flower 4-5 $\times 1.5-2 \mathrm{~mm}$, in fruit up to $10 \times 3 \mathrm{~mm}$. Corolla pale blue to lilac, $1 \frac{1}{2}-2 \times$ as long as the calyx, cup-shaped, 7-10 $\mathrm{mm}$ long; tube somewhat shorter than the calyx, 3-4 mm long; limb actinomorphic or subactinomorphic; lobes oval. Stamens equal, 5 or 4 with a staminode, included or hardly so. Seeds $1 \frac{1}{2}-2 \times$ as long as wide, $0.25-0.3 \times 0.15-0.2 \mathrm{~mm}$, obliquely striate or reticulate-striate.

Plant 5-15. cm high. Stems very short, $0.5-6 \mathrm{~cm}$ long. Leaves subsessile, $2-16 \times$ $0.7-6 \mathrm{~cm}$, membranaceous or papyraceous when dry; primary veins 6-8 pairs. Inflorescence up to $15 \mathrm{~cm}$ long. Peduncle slender, sparsely pilose, $1-6 \mathrm{~cm}$ long. Bracts lanceolate, $3-5 \times$ as long as wide, acuminate, entire, pilose to glabrous. Pedicels slender, sparsely pilose, $1-4 \mathrm{~cm}$ long. Sepals sparsely pilose outside, glabrous inside. Corolla glabrous on both sides; lobes 4-6 $\times$ 3-4 mm. Filaments somewhat longer than the ovary, $2 \mathrm{~mm}$ long, widened at the base; anthers reniform, $0.5-0.75 \times$ 1-1.2 mm; cells divergent at the base. Staminode half as long as the stamens, $1.2 \mathrm{~mm}$ long; sterile anther very small. Ovary ovoid, $2 \times 1.5 \mathrm{~mm}$; style curved, glabrous, 3-4 mm long; stigma obscurely saucer-shaped. Capsule about half as long as the calyx, ovoid, $3-4 \times 2.5-3 \mathrm{~mm}$, acuminate at the apex. Seeds medium brown, mucronate at the base.

Distribution: Trinidad and Tobago.

Ecology: On moist rocky banks, in rainforests; at low (?) and middle elevations.

Trinidad: Blanchisseuse, herb. Kuntze 831 (NY); Blanchisseuse Road, Broadway 6738 (K, MO, S,.US); Mt. Tucuche, Othmer 217 (M); ibid. Britton, Hazen \& Mendelson 1277 (NY, GH, US); Maracas, Broadway, I June 1928 (K), 13 March 1927 (BM); ibid., herb. Trin. 2506 (US); Maraval, Broadway, 1 Jan. 1892 (NY); Cauro, Broadway, 24 June 1934 (K); Las Cuevas, Crueger s.n. (GOET, holotype, isotype: K); northwest of Piedra Blanca, Simmonds 301 (US) ; Morne Bleu, Britton, Freeman \& Bailey 2247 (NY, G, GH, K, US); Ortinola Estate, Britton \& Hazen 252 (NY, GH, US); La Canoa Road, Santa Cruz, Broadway 2291 (MO); Fendler 506 (BM, K).

Toвago: Campbleton, near Charlotte Ville, Broadway 3132 (BM, G, L, MO, NY); Main Ridge above Parlatuvier, Sandwith 1915 (K, NY), 1922 (K, with spirit coll., stamens 5 or 4 with a staminode!).

Excluded species:

$\mathcal{N}$. repens Donn. Sm., Bot. Gaz. 31: 118. $1901=$ Phinaea repens (Donn. Sm.) Solereder.

$\mathcal{N}$. saxicola Brandegee, Univ. Calif. Publ. Bot. 6: 65. $1914=$ Niphaea sp.?

Linden 995 cited as Napeanthus sp. by Bentham and Hooker (1876, p. 1018) belongs to an undescribed genus related to Episcia and Cremosperma. The BR sheet has a strongly zygomorphic nearly trumpetshaped corolla, coherent anthers, and a dorsal disk gland. 


\section{REVISION OF THE GUIANAN GESNERIACEAE}

\section{Diagnose of THE Family ${ }^{1}$ )}

Terrestrial or epiphytic shrubs, subshrubs, herbs, or vines, or rarely lianas or small trees, sometimes tuber-bearing. Stems often fleshy. Leaves cauline or less often radicular, usually opposite, mostly petiolate; blade mostly rather fleshy, membranaceous to coriaceous when dry, mostly dentate, usually pinnately veined, usually conspicuously hairy with septate hairs. Inflorescence mostly axillary, sometimes terminal. Flowers bisexual, solitary, fasciculate, or in pedunculate umbels or cymes, pedicellate. Sepals green or coloured, valvate or imbricate in the bud, free or connate, sometimes entirely united, herbaceous, sometimes toothed. Corolla variously coloured, tubular or rarely campanulate or rotate, erect, oblique, or horizontal in the calyx, often spurred or gibbous at the base, contracted in the throat or not; limb typically 5-lobed, actinomorphic to strongly bilabiate; lobes imbricate in the bud. Stamens included or exserted, mostly 4 (in Guianan spp.), sometimes 2 , or rarely 5 ; if 4 didynamous or rarely equal, if 5 equal; filaments inserted on the corolla, mostly on the base, connate at the base or not; anthers 2-celled, all coherent, coherent in pairs, or if free mostly connivent; cells parallel or divergent at the base, confluent at the apex or not, dehiscent throughout or only partially. Staminodes wanting, one (then flowers 4-staminate), or three (in some 2-staminate flowers), often very small, with or without sterile anther. Ovary superior or often partially inferior, often hairy, 1-celled; style 1, simple, included or exserted; stigma bilobed, stomatomorphic, or capitate or nearly so, pubescent with glandular hairs. Disk annular, surrounding the ovary, composed of $2-5$ glands, or reduced to a single dorsal gland. Placentae 2 , parietal; mostly bilobed. Ovules numerous, anatropous ${ }^{2}$ ). Fruit a berry or a capsule, if a capsule usually loculicidal and bivalved or also septicidal and 4-valved. Valves membranaceous to sublignose or fleshy. Seeds numerous, small, mostly obliquely striate or reticulate-striate, with endosperm ${ }^{3}$ ). Embryo straight; cotyledons short; rootlet turned to the hilum.

Distribution: About 1800 species in about 140 genera, mainly in tropical and subtropical regions.

1) Diagnose of the family mainly based on American representatives.

2) Ovules analysed in herbarium specimens of Chrysothemis pulchella and Napeanthus primulifolius, and in living plants cultivated in the greenhouse at Utrecht of Chirita lavandulacea, Columnea gloriosa, Kohleria amabilis, Saintpaulia ionantha, and Smithiantha hybr.

$\left.{ }^{3}\right)$ Interior structure of seeds analysed in herbarium specimens of Alloplectus cristatus var. brevicalyx, Besleria lutea, Chrysothemis friedrichsthaliana, rupestris, Codonanthe calcarata, crassifolia, Columnea aureonitens, oerstediana, Drymonia serrulata, Episcia maculata, Gloxinia perennis, Kohleria spicata, Napeanthus primulinus, rigidus, subacaulis, and Rechsteineria allagophylla. 
Key to the genera (based on Guianan species only) ${ }^{1}$ ):

1. Leaves radicular or nearly so, solitary, several together, or rosulate; when stems present peduncle conspicuous, mostly longer than the petioles . . . . . . . . .

Leaves cauline, mostly opposite; when stems very short peduncle mostly obsolete . . . . . . . . . . . . .

2. Leafblades lanceolate or oblong-spathulate; stems short or very short . . . . . . . . . . . . . .

Leafblades rounded, ovate, or oblong-ovate; no stems . .

3. Leaves sessile or subsessile; inflorescence with small bracts; fruiting calyx strongly many-nerved; corolla not spurred; no disk ..... 11. Napeanthus, p. 420

Leaves petiolate; inflorescence without bracts; calyx not strongly nerved; corolla spurred; disk glands 5 . . .

15. Tylosperma, p. 432

4. Corolla tubular, spurred at the base; leafblades often proliferous at the margin . . 14. Rhoogeton, p. 429

Corolla campanulate, not spurred at the base; leafblades not proliferous . . . 10. Lembocarpus, p. 418

5. (1). Ovary inferior; terrestrial, mostly herbaceous plants with rhizomes

Ovary superior, or when not quite superior corolla bilabiate and stamens exserted; sometimes epiphytic . . . . .

6. Stems and leaves glabrate; corolla campanulate, violetblue . . . . . . 8. Gloxinia, p. 415

Stems and leaves densely hairy; corolla tubular, orange or red . . . . . . . 9. Kohleria, p. 416

7. Inflorescence with large bracts, several-flowered, pedunculate; bracts larger than the sepals, at least the outer ones

Inflorescence with or without small bracts . . . . . .

8. Bracts purple or dark red; leafblades entire; adventitious roots when present spreading .

. . . . . . 1.1. Alloplectus coccineus, p. 359

Bracts green, purple- or dark red-veined; leafblades serrate; adventitious roots in one unilateral line.

7.6. Episcia maculata, p. 407

9. Calyx red, wide in proportion to the corolla; sepals completely united or nearly so; inflorescence pedunculate, umbellate, seemingly terminal; terrestrial herbs with tubers . . . . . . . 3. Chrysothemis, p. 375

Calyx green or coloured; sepals never completely united, when nearly so inflorescence sessile (Besleria insolita). .

10. Corolla red, $3-5 \times$ as long as the calyx, conspicuously bilabiate; dorsal lip erect, ventral lip spreading; stamens exserted; anthers all coherent, covered by the dorsal lip

Corolla not or obscurely bilabiate; stamens exserted when corolla not red . . . . . . . . . . . . .

1) See the remark on p. 314 . 
11. Erect terrestrial herbs with tubers; flowers axillary or in a terminal thyrsus; the base of the ovary adnate to the base of the calyx; fruit a sublignose capsule, dehiscent . . .

13. Rechsteineria, p. 424

Creeping, climbing or pendant mostly epiphytic subshrubs, vines, or herbs with spreading adventitious roots; ovary completely superior; fruit a berry, indehiscent . . . .

5. Columnea (sect. Columnea), p. $38 \dot{2}$

12. Corolla infundibuliform, $3-6 \times$ as long as the calyx, spurred at the base; anther cells subglobose, separated by the enlarged connective; leaves fleshy, about $4-7 \mathrm{~cm}$ long; plant glabrous or nearly so, epiphytic on ants' nests

4. Codonanthe, p. 378

Corolla not infundibuliform, otherwise plant usually conspicuously hairy. . . . . . . . . . .

13. Peduncle short or long, but conspicuous, never hidden by hairs; bracts when present not conspicuously hairy; if flowers solitary sepals entire or nearly so, not serrate

Peduncle obsolete or very short; flowers 1-many, axillary

14. Inflorescence congested-paniculate; flowers numerous, small; sepals rounded, $2-3 \mathrm{~mm}$ long; corolla $5 \mathrm{~mm}$ long . . . .

2.1. Besleria flavo-virens, p. 366

Inflorescence not congested; sepals not rounded; corolla more than $8 \mathrm{~mm}$ long . . . . . . . . . .

15. Corolla trumpet-shaped; inflorescence with small bracts; disk gland 1, dorsal . . . . . . 7. Episcia, p. 398

Corolla cylindric or nearly so; inflorescence without bracts; disk annular . . . . . . 2. Besleria, p. 365

16. (13). Flowers aggregated; sepals not linear, acuminate, entire; corolla tube cylindric or nearly so, glabrous outside; anthers reniform; no bracts 2. Besleria, p. 365

Flowers 1-many; when aggregated sepals not entire . . . 17

17. Corolla lobes erect and/or small; corolla yellow or yellowish Lobes not small, otherwise corolla white or red . . . . .

18. Leaves of a pair strongly unequal, the larger one about $10 \times$ as long as the other.

5. Columnea (sect. Collandra), p. $38 \dot{2}$

Leaves of a pair equal or unequal, when unequal the larger one up to $2 \times$ as long as the other . . . . . . . .

19. Sepals - oblong-spathulate, $4-4.5 \times$ as long as wide; leafblades lanceolate or oblong-elliptic; plant hirsute . . .

5.2. Columnea calotricha, p. 385

Sepals ovate, up to $2 \times$ as long as wide; leafblades elliptic or oblong-elliptic

1. Alloplectus (sect. Alloplectus), p. 358

20. Corolla amply tubular, horizontal in the calyx, limb large; sepals green, mostly large, about $2-4 \mathrm{~cm}$ long; $1-4 \times$ as long as wide, mostly epiphytic subshrubs or vines . . . .

6. Drymonia, p. 391 
Corolla trumpet-shaped or infundibuliform; mostly terrestrial herbs, sometimes shrubby .........

21. Sepals mostly long-acuminate, villose outside; corolla creamy or white, never red; leaves of a pair equal or subequal; no stolons; anther cells parallel 12. Nautilocalyx, p. 422 When sepals acuminate corolla red, or leaves of a pair strongly unequal and the smaller one often wanting, or corolla at least partially mauve, or anther cells divergent at the base . . . . . . . 7. Episcia, p. 398

1. Alloplectus Mart., Nov. Gen. 3: 53. 1829.

Epiphytic or terrestrial shrubs, subshrubs, vines, lianas, or herbs of very different size, when climbing up to $9 \mathrm{~m}$ long, when erect up to $2 \mathrm{~m}$ high. Stems erect, ascending, creeping, or climbing, branched or not, mostly with spreading adventitious roots. Leaves opposite, those of a pair equal, subequal, or rarely unequal, often rather longpetiolate; blade usually elliptic. Flowers axillary, solitary, fasciculate, or in pedunculate umbels or racemes with often branched axes. Sepals 5, usually coloured, free or nearly so. Corolla variously coloured, erect or horizontal in the calyx, contracted in the throat; limb narrow, erect or spreading, lobes 5 , subequal, rounded, usually entire. Stamens 4, didynamous, included; filaments connate at the base, inserted on the base of the corolla; anthers commonly free, oblong to quadrate or broader than long; cells parallel, discrete, sometimes sagittate at the base, sometimes only partially dehiscent. Ovary superior; style included; stigma bilobed, stomatomorphic, capitate, or nearly so. Disk glands $1-5$, mostly one large dorsal one. Placentae ovuliferous only on the inner or on both surfaces. Fruit a berry-like capsule, bivalved; valves usually thick, coriaceous. Seeds shining, fusiform or ellipsoid, longitudinally or obliquely striate.

Type species: $A$. hirtellus (Schott) Preston (A. sparsiforus Mart.).

Distribution: about 80 species in tropical America.

Key to the species:

1. Leafblades and sepals serrate; flowers mostly solitary; anthers not sagittate (sect. Alloplectus) . . . . . . . . . . .

Leafblades entire; flowers arranged in inflorescences with large purple or dark red bracts; anthers sagittate at the base (sect. Macrochlamys (Dcne.) Fritsch) . . . 1. A. coccineus

2. Leaves of a pair unequal, the larger one about twice as long as the other; corolla about as long as the calyx ... . . . .

Leaves of a pair subequal; corolla about twice as long as the calyx

3. Flowers solitary; pedicels long, often almost equalling the leaves . . . . . . . . . . . 2. A. cristatus

Flowers fasciculate; pedicels much shorter than the leaves 
1. A. coccineus (Aubl.) Mart. ex G. Don, Gen. Syst. 4: 655. 1838 (see also Martius, Nov. Gen. 3: 57. 1829); D.C., Prod. 7: 545. 1839; Hanstein, Linnaea 34: 366. 1865; Lemée, Fl. Guian. fr. 3: 463.1953.

Basionym: Besleria coccinea Aubl., Hist. Pl. Guian. 2: 632. 1775.

Type: French GuiANA: banks of Sinnemary R., 20 miles from the sea coast, Aublet s.n. (BM).

Homotypic synonym: Crantzia coccinea (Aubl.) Fritsch in Engl. Prantl, Nat. Pflanzenf. 4 (3b): 168. 1894 ("1895").

Heterotypic synonyms: Alloplectus circinatus Mart., Nov. Gen. 3: 56, t. 223. f. 2. 1829; G. Don, Gen. Syst. 4: 655. 1838; D.C., Prod. 7: 545. 1839; Hanstein, Linnaea 26: 177, 209, 215, t. 2. f. 50A. 1853; in Martius, Fl. Bras. $8(1): 406$. 1864; Linnaea 34:366. 1865. Lectotype: BRAzIL: Amazonas, near Madeira R., Martius herb. 75 (M, photographs: U, US).

Alloplectus patrisii D.C., Prod. 7: 545. 1839; Bentham, Lond. Journ. Bot. 5: 362. 1846; Schomburgk, Reisen Br. Guian. 3: 972. 1848; Hanstein, Linnaea 34: 365. 1865; Lemée, Fl. Guian. fr. 3: 463.1953.

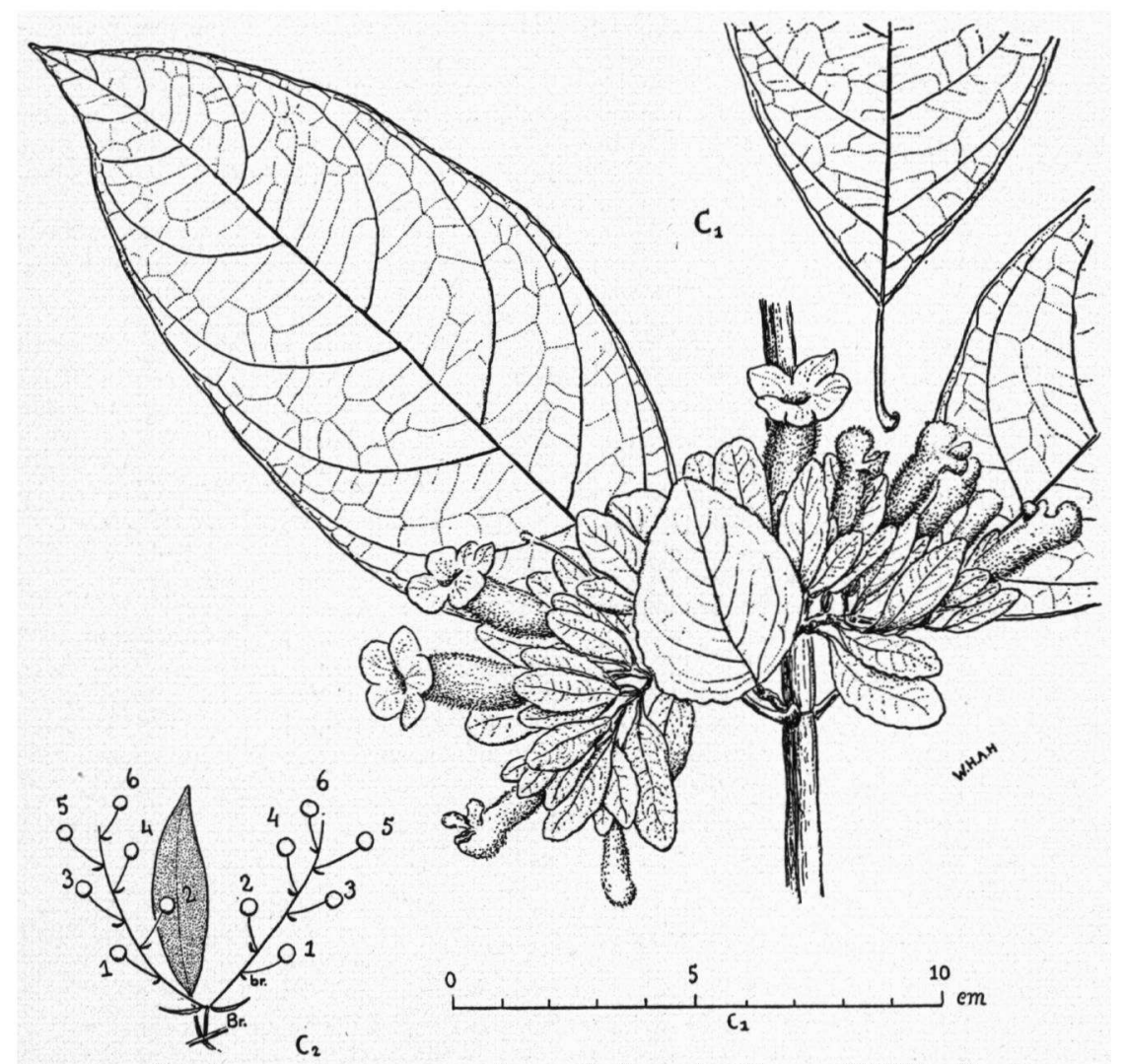

Fig. 7. Alloplectus coccineus var. coccineus: $\mathrm{C}_{1}$. branch (Cowan 38361); $\mathrm{C}_{2}$. diagram of inflorescence. 
Type: French Gutana: Cayenne, Patris s.n. (G-DC, 4 sheets [one number ?], photographs of first: F, GH, US). Homotypic synonym: Crantzia patrisii (D.C.) Fritsch in Engl. Prantl, Nat. Pflanzenf. 4 (3b): 168. 1894 ("1895"); in Engl., Bot. Jahrb. 37: 486. 1906; Pulle, Enum. 420. 1906.

Leaves of a pair subequal or unequal, the larger one up to $2 \frac{1}{2} \times$ as long as the other; blade entire or sometimes obscurely denticulate near the apex, sparsely appressed-pubescent to glabrous on both sides. Inflorescence racemose, often with branched axis, provided with large purple or dark red bracts covering the sepals, these coloured like the bracts or somewhat paler, obovate, obtuse or rounded at the apex, narrowed towards the base. Anthers mostly connivent, sagittate at the base.

Epiphytic or terrestrial shrub, subshrub, vine, or liana, when climbing up to $8 \mathrm{~m}$ long, when erect $0.60-2.50 \mathrm{~m}$ high. Stems sarmentose, branched or not, sometimes with spreading adventitious roots, puberulous at the apex, glabrous and shining. Leaves: petiole sparsely appressed-pubescent, $0.5-6$ (mostly 1-4) cm long; blade dark green above, paler green and purple- or dark red-veined beneath, obliquely elliptic, oblong-elliptic, or oblong, variable, $2 \frac{1}{2}-3 \frac{1}{2} \times$ as long as wide, $2.7-29 \times 1.8-10 \mathrm{~cm}$ (mostly about 8-15 $\times 3-6 \mathrm{~cm}$ ), fleshy, papyraceous or subcoriaceous when dry, acuminate at the apex, cuneate to almost decurrent at the base. Inflorescence: peduncle sparsely appressed-pubescent. Flowers 1-10 or more together. Bracts purple, dark red, or pinkish-red, the inner ones mostly paler, sometimes yellowish-white, leafy, veined, orbicular, ovate, or obovate, $1-1 \frac{1}{2} \times$ as long as wide, $15-43 \times 15-43 \mathrm{~mm}$, acute, obtuse, rounded, or apiculate at the apex, cuneate, rounded, or subcordate at the base (in the same specimen!), entire, sinuate, or crenulate, sparsely pubescent on both sides, the two outer ones mostly larger than the others, orbicular. Sepals purple, dark red, pinkish-red, or sometimes yellowish-white, free, subequal, leafy, veined, $1 \frac{1}{2}-2 \frac{1}{2} \times$ as long as wide, 13-25 $\times$ 6-15 mm, entire, sinuate, or crenulate, sparsely appressed-pubescent on both sides. Corolla horizontal in the calyx, $1 \frac{1}{2}-3 \times$ as long as the calyx, 30-48 $\mathrm{mm}$ long, at the base $4-6 \mathrm{~mm}$, at the throat $6-12 \mathrm{~mm}$ wide, spurred at the base, slightly contracted above the spur and in the throat, not conspicuously ventricose, puberulous to villose outside, sometimes glabrous at the base, inside with an area of glandular hairs dorsally in the tube; tube nearly cylindric, 28-35 mm long; spur obtuse, $3 \times 3-5 \times 5 \mathrm{~mm}$; limb spreading, 8-26 mm wide; lobes subequal, rounded, entire or sinuate, $4-10 \mathrm{~mm}$ in diam. Filaments glabrous, contorted at the apex, all connate and widened at the base, inserted on the base of the corolla; anthers free, oblong, 5-6 $\times 2 \mathrm{~mm}$; cells dehiscent by a longitudinal split starting at the base. Staminode filiform, glabrous, curved through the spur, without anther. Ovary appressed-pubescent, ovoid, 4-6 $\times 3-5 \mathrm{~mm}$; style appressed-pubescent, curved; stigma capitate. Disk gland one, dorsal, large, entire or obscurely bilobed, glabrous. Placentae ovuliferous only on the inner or on both surfaces. Capsule yellow (teste Aublet), subglobose, sparsely appressed-pubescent, 13-15 $\times 9-10 \mathrm{~mm}$, bivalved, with a thickened ring along the line of dehiscence; valves coriaceous. Seeds dark brown (?), fusiform, longitudinally striate, $1 \times 0.2 \mathrm{~mm}$.

Distribution: Guiana and northern Brazil.

Ecology: in rainforests; alt. 0-600 m.

\section{A. coccineus var. coccineus}

Fig. 7, p. 359

Corolla yellow, usually not spotted. Basal part of peduncle below the bracts very short, much shorter than the outer bracts, mostly about one-fifth (from 0.11 to 0.36 ) of the length of the outer bracts.

Distribution: Guiana, northern and central Brazil. 
Brttish Gutana: Itabu Ck., Beddington 35 (K), 45 (K); Basin of Kuyuwini R., Essequibo trib., A. C. Smith 2531 (NY); Basin of Shodikar Ck., Essequibo trib., A. C. Smith 2880 (NY, US), 2887 (NY, A, F, G, K, MO, S, U, US); Upper Essequibo R., Mataruki R., Meyers 5827 (K); Schomburgk 110 (K).

Suriname: Moengo, Jonker 486 (U) ; vic. of Moengo, Cottica R., Cowan 38985 (U). French Guiana: Marowijne R., Mélinon 109 (P), 342 (P), 434 (P, U); ibid:, Wachenheim $146(\mathrm{P})$; near mouth of Mana R.; Acarouany R., Benoist 196 (P)'; ibid., Sagot 425 (P, BM, G, K, S, U, W); banks of Sinnemary R., Aublet s.n. (BM, holotype); near Montagne Anglaise, L. G. Richard s.n. (P); Roura, Soubirou s.n. (P); Mt. Kaw, Cowan 38734 (U); Lower Oyapock R., Geay 334 (P); De Candolle et l'Héritier s.n. (G); herb. expos. col. 62 (P), s.n. (P); Gabriel, anno 1802 (G); Leblond 284 (G); herb. Maire s.n. (P); Martin s.n. (P, U); Martin, herb. Rudge s.n. (BM); Patris s.n. (G-DG, type of A. patrisii); Poiteau s.n. (K, W). BRAzIL: AmAPÁ: Rio Amapari, Serro do Navio, Cowan \& Maguire 38072 (US); ibid., Cowan 38258 (US), 38310 (US), 38361 (US), 38393 (US), 38513 (US).

\section{A. coccineus var. fusco-maculatus Lwbg., p. 300 . Fig. 8, p. 362}

Type: Suriname: Nassau Mts., Cowan \& Lindeman 39049 (U).

Corolla creamy, yellow, or white, brown-purple- or reddish-brownspotted in the throat or on the limb. Basal part of peduncle below the bracts on the average 1.2 (from 0.4 to 2.2 ) $\times$ as long as the outer bracts, up to $60 \mathrm{~mm}$ long.

Distribution: Suriname.

SURINAmE: Corantijn R., Winana, Kaboeri Ck., B.W. 2987 (U); Emmaketen, B.W. 5653 (U); Lucie R., B.W. 7103 (U); Tosso Ck., Tapanahoni R., Florschütz 359 (U); Placer Lawa, B.W. 4115 (U); banks of Gonini R., Versteeg (U,, 2 sheets); Upper Litanie R., Tumuc Humac Range, Rombouts 828 (U); Brownsberg, B.W. 6328 (U); near Guyana Goudplacer, Boldingh $3918 \mathrm{e}$ (U); ibid., coll. indig. 99 (U); near Plant. Berlijn in Para distr., Splitgerber 716 (L); Jodensavanne-Mapane Ck. area, Suriname R., Lindeman 4987 (U); Suriname R., near Bergendaal, Focke 975 (U); Suriname R.; near Victoria Station, Hostmann \& Kappler 1372 (G, MO, P, 2 sheets, S, U, W, 2 sheets); banks of Perica R., Wullschlägel 1322 (BR, GÓET, U); near Maripaston, Saramacca R., Kegel 1370 (GOET); Nassau Mts., Lanjouw \& Lindeman 2096 (U) ; ibid., Cowan \& Lindeman 39049 (U, holotype), 39179 (U); ibid., Maguire 40702. (U); Hostmann 1225 (K, 2 sheets).

2. A. cristatus (L.) Mart. ex G. Don, Gen. Syst. 4: 655.1838 (see also Martius, Nov. Gen. 3: 57. 1829); D.C., Prod. 7: 545. 1839; Grisebach, Fl. Br. W.I. Isl. 463. 1861 ("1864"); Hanstein, Linnaea 34: 375. 1865; Duss, Fl. Ant. fr. 432. 1897; Urban, Symb. Ant. 2: 356. 1901; in Fedde, Repert. Beih. 5: 47. 1920; Morton, Contrib. U.S. Nat. Herb. 29: 17. 1944.

Basionym: Besleria cristata L., Sp. Pl. 619. 1753; Jacquin, Sel. Stirp. Amer. Hist. 188, t. 119. 1763.

Neotype: Burmann, Plant. Amer. Car. Plumier. 1756. t. 50. Homotypic synonyms: Crantzia cristata (L.) Scop., Intr. 173. 1777; Fritsch in Engl. Prantl, Nat. Pflanzenf. 4 (3b): 168. 1894 ("1895"). Prionoplectus cristatus (L.) Oerst., Centralamer. Gesn. 78, t. 9. f. 36-40. 1858.

Lophia phoenicea Desv. ex Hamilton, Prod. P1. Ind. Occ. 47. 1825.

Leaves of a pair equal or subequal; blade serrate or crenate-serrate, strigose above. Flowers solitary. Pedicel long, mostly somewhat shorter than the leaves. No bracteoles. Sepals dark red, broadly ovate, 
cordate at the base. Corolla yellow. Anthers broader than long, all coherent.

Terrestrial or epiphytic subshrub. Stems usually creeping, sarmentose, branched, with spreading adventitious roots, glabrescent. Leaves: petiole densely strigose or subtomentose, 3-35 mm long; blade dark green above, paler beneath, oblongelliptic or oblong-ovate, $1 \frac{1}{2}-3$ (mostly 2 ) $\times$ as long as wide, papyraceous when dry, acuminate at the apex, cuneate or rounded at the base. Sepals connate at the base for about $3 \mathrm{~mm}$, erect, strigillose on both sides, in fruit sometimes slightly enlarged, four subequal, $1-1 \frac{1}{2} \times$ as long as wide, the fifth (dorsal) somewhat smaller and narrower. Corolla nearly cylindric, oblique in the calyx, $1 \frac{1}{2}-2 \times$ as long as the calyx, 25-35 mm long, at the base $3-4 \mathrm{~mm}$, at the throat $5-8 \mathrm{~mm}$ wide,

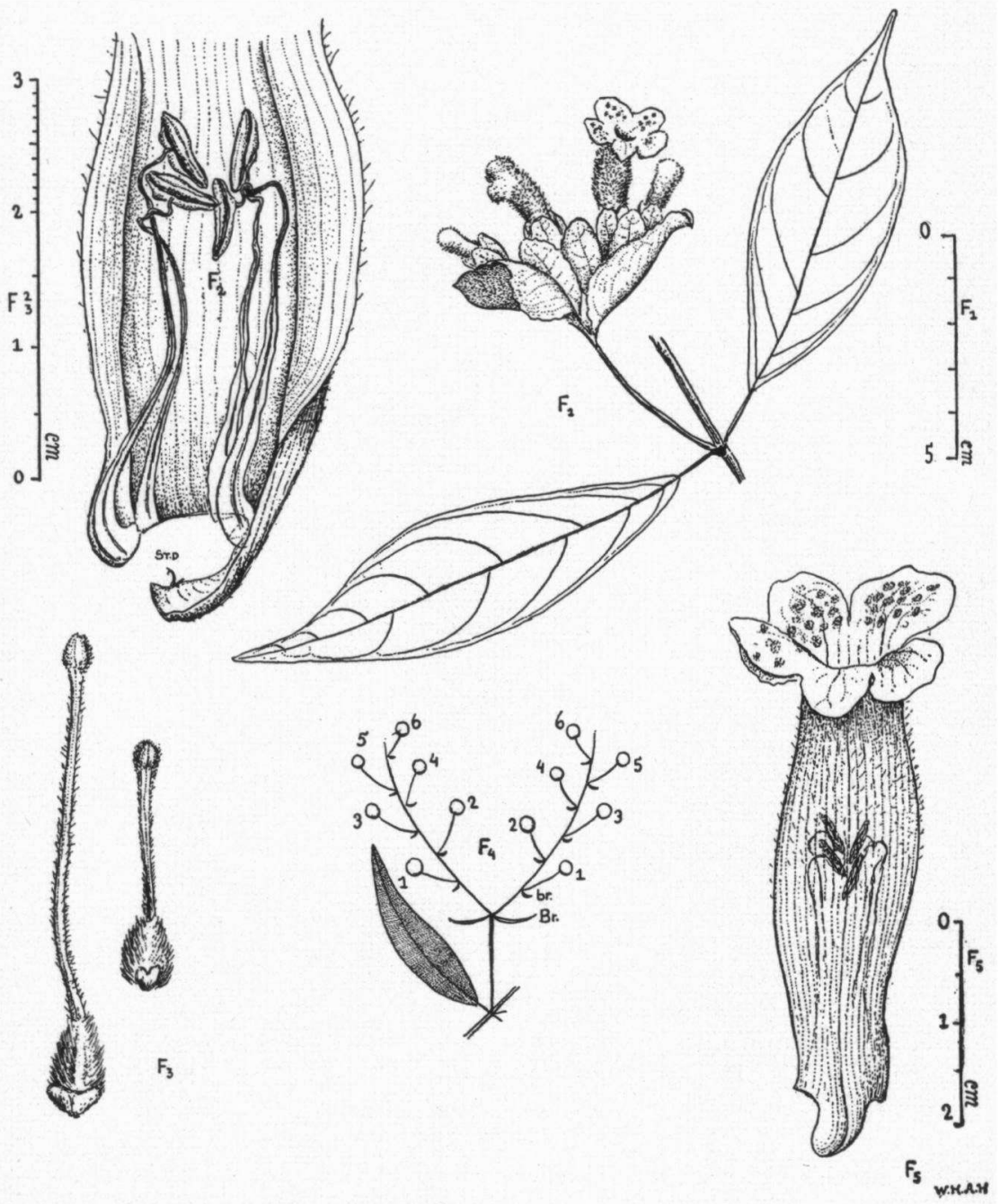

Fig. 8. Alloplectus coccineus var. fusco-maculatus: $F_{1}$. branch (Versteeg 253); $F_{8}$. opened corolla (Cowan 39049); $F_{3}$. pistil, extremes of length (left: Cowan 39049, right: Coll. indig. 99); $F_{4}$. diagram of inflorescence; $F_{5}$. corolla (Cowan 39179). 
shortly spurred at the base, not or scarcely contracted above the spur and in the throat, hardly ventricose, densely pilose outside, especially above, glabrous inside; tube 20-25 mm long; limb spreading, 9-14 $\mathrm{mm}$ wide; lobes subequal, rounded, minutely dentate, $2.5-4 \mathrm{~mm}$ in diam. Filaments glabrous, contorted or not, all connate at the base for about $4 \mathrm{~mm}$, inserted on the base of the corolla; anther cells dehiscent throughout. Staminode small, $2 \mathrm{~mm}$ long, glabrous, without anther. Ovary appressed-pubescent, ovoid, 4-5 $\times 3 \mathrm{~mm}$; style about as long as the stamens, glabrous; stigma stomatomorphic. Disk gland one, dorsal, entire, glabrous. Placentae ovuliferous on the inner surface only. Capsule globose, sparsely appressedpubescent, $9-10 \mathrm{~mm}$ in diam., bivalved. Seeds dark brown, ellipsoid, about $2 \times$ as long as wide, $0.8-1 \times 0.3-0.5 \mathrm{~mm}$, obliquely striate.

Distribution: Lesser Antilles and Guiana.

In Guiana only one variety:

A. cristatus var. epirotes Lwbg., p. 298.

Type: British Guina: Mt. Ayanganna, Pakaraima Mts., in montane rainforest, 1000-1500 $\mathrm{m}$ alt., Maguire, Bagshaw \& Maguire 40594 (NY, holotype; isotypes: U, US).

Sepals sharply dentate-serrate, acute at the apex, in flower about $22 \times 20 \mathrm{~mm}$. Leafblades about $2 \frac{1}{2} \times$ as long as wide, $5-10 \times 2-4.5$ $\mathrm{cm}$, slightly acuminate at the apex, rounded at the base or nearly so, crenate-serrate, hirsutulous beneath, especially on the midrib and veins. Pedicel red, hirsutulous, 5-8 cm long. Stems hirsutulous at the apex.

Distribution: Once collected in British Guiana.

British Gutana: Mt. Ayanganna, Pakaraima Mts., Maguire, Bagshaw \& Maguire 40594 (NY, holotype; isotypes: U, US).

Notes : One additional specimen of $A$. cristatus is known from Guiana, which belongs to A.c. var. cristatus, differing mainly from the above variety by its laciniate and serrate sepals.

French Guiana: Leblond s.n. (P-LA).

Its locality is somewhat doubtful as this variety is otherwise only known from Martinique and St. Lucia.

The specimen identified by Aublet (1775. D. 637) as A. (Besleria) cristatus is Besleria flavo-virens. SchomburgK's specimen $(1848$, p. 972) has been destroyed in Berlin.

3. A. meridensis Klotzsch ex Hanst., Linnaea 34 : 372 . 1865; Morton, Fieldiana 28: 520. 1953.

Type: Venezuela: Mérida, Moritz 1132 ("1032") (BM, BR, L, isotypes).

Leaves of a pair equal or subequal ; blade serrate, strigillose above, hirsute or subtomentose beneath, especially on the midrib and veins. Inflorescence sessile, with several often deciduous bracts, severalflowered. Pedicels much shorter than the leaves. Sepals red or rosered, ovate, obtuse or acute at the apex, cordate at the base, serrate, with recurved margins. Corolla tube rose-red, with 2 red dorsal and 3 yellow ventral lobes.

Terrestrial or epiphytic(?) herb or shrub, $0.30-1.80 \mathrm{~m}$ high. Stems sarmentose, erect, ascending, or climbing, sometimes with spreading adventitious roots, hirsute or subtomentose at the apex, glabrescent. Leaves: petiole hirsutulous or subtomentose, 12-60 mm long; blade dark green above, paler green or red beneath, often obliquely oblong-elliptic, $1 \frac{1}{2}-3$ (mostly $2-2 \frac{1}{2}$ ) $\times$ as long as wide, $7-20 \times 3-12 \mathrm{~cm}$, papyraceous when dry, acuminate at the apex, cuneate or rounded at the base. 
Inflorescence: bracts red, sometimes green at the base, oblong, obtuse or rounded at the apex, sinuate, virtually glabrous above, hirsutulous beneath. Pedicels hirsutulous, 6-40 mm long. Sepals free, about as long as wide, 12-18 mm long, hirsutulous outside, expecially in the middle, pilose inside. Corolla oblique in the calyx, $1 \frac{1}{2}-2 \times$ as long as the calyx, 20-29 $\mathrm{mm}$ long, at the base $4 \mathrm{~mm}$, at the throat $6 \mathrm{~mm}$ wide, shortly spurred at the base, contracted above the spur and in the throat, ventrally ventricose above and there $6-9 \mathrm{~mm}$ wide, villose outside, inside with glandular hairs in the throat; tube 16-20 mm long; limb spreading, about $7-10 \mathrm{~mm}$ wide; lobes subequal, rounded, crenate-serrate, $1.5-3 \mathrm{~mm}$ in diam. Filaments glabrous, contorted, all connate at the base, inserted on the base of the corolla; anthers orbicular, coherent (?); cells dehiscent throughout by a longitudinal split. Staminode small, linear, glabrous, $2 \mathrm{~mm}$ long, with an obscure sterile anther. Ovary hirsute, ovoid, $4 \times 3 \mathrm{~mm}$; style glabrous; stigma bilobed(?). Disk gland one, dorsal, entire, glabrous. Placentae ovuliferous on the inner surface only. Fruit and seeds not seen.

Distribution: Colombia and Venezuela:

Ecology: In montane rainforests, at high elevations.

Description based on Colombian and Venezuelan material. From Guiana only known by a vegetative specimen with note: "Calyx red":

Suriname: Wilhelmina Mts., B.W. 7092 (U).

This specimen might be $A$. ichthyoderma Hanst. which differs mainly by its entirely yellow corolla, or another species.

\section{A. savannarum Morton, Bull. Torr. Bot. Cl. 75: 563. 1948.}

Type: British Guiana: Kaieteur, Maguire \& Fanshawe 23127 (NY, holotype, isotypes: $\mathrm{BR}, \mathrm{F}, \mathrm{G}, \mathrm{K}, \mathrm{MO}, \mathrm{U}, \mathrm{UC}$ ).

Leaves of a pair mostly unequal, the larger one mostly about $2 \times$ as long as the other; blade serrate, shortly pilose above, hirsute beneath. Flowers solitary or 2 together. Bracts very small. Pedicels short, shorter than the petioles. Sepals vermilion or scarlet, ovate, largely dentate-serrate, entire near the acute apex, subcordate at the base. Corolla yellowish, oblique, curved.

Epiphytic vine, up to $5 \mathrm{~m}$ high. Stems terete, branched or not, yellow-tomentose near the apex, glabrescent. Leaves: petiole yellow-hirsute, $2-5.5 \mathrm{~cm}$ long; blade medium green above, paler beneath, obliquely oblong-elliptic, papyraceous when dry, shortly acuminate at the apex, very obliquely cuneate at the base, in the larger leaf of a pair about $2 \frac{1}{2} \times$ as long as wide, $7 \frac{1}{2}-16 \times 3-7 \mathrm{~cm}$, in the smaller one about $2 \times$ as long as wide, $2 \frac{1}{2}-7 \mathrm{~cm} \times 1-4 \mathrm{~cm}$. Inflorescence: pedicels hirsute, 9-12 mm long. Bracts linear, hirsute, about 5-10 $\times \frac{1}{2} \mathrm{~mm}$. Sepals free, subequal, erect, about $2 \times$ as long as wide, $2-3 \times 1-1 \frac{1}{2} \mathrm{~cm}$, hirsute on both sides. Corolla not or hardly gibbous at the base, $20-28 \mathrm{~mm}$ long, at the base $5 \mathrm{~mm}$ wide, upwards ampliate, up to $10 \mathrm{~mm}$, contracted in the throat and there $5 \mathrm{~mm}$ wide, outside glabrous at the base, upwards densely yellow-sericeous, inside pilosulous towards the apex; limb small, erect; lobes equal, triangular, rounded at the apex, about $2 \mathrm{~mm}$ long and wide. Filaments glabrous, hardly contorted, all connate at the base for $8 \mathrm{~mm}$, inserted on the base of the corolla; anthers nearly orbicular, all coherent; cells dehiscent throughout by a longitudinal split. Staminode very small, about $0.7 \mathrm{~mm}$ long, glabrous, with a triangular sterile anther. Ovary hirsute with long hairs, ovoid, about $3 \times 2 \frac{1}{2} \mathrm{~mm}$; style short, glabrous; stigma bilobed. Disk gland one, dorsal, large, emarginate, glabrous. Placentae ovuliferous on the inner surface only. Capsule subglobose, hirsute, about $12 \mathrm{~mm}$ long, with two thick valves. Seeds reddish, fusiform, about $5 \times$ as long as wide, about $1.5 \times 0.3$ $\mathrm{mm}$, longitudinally striate.

Distribution: Guayana Highlands.

Ecology: In rainforests on mountain slopes; alt. 400-1500 m. 
British Gulana: Kaieteur, Maguire \& Fanshawe 23127 (NY, holotype, isotypes: BR, F, G, K, MO, U, UC); ibid., Sandwith 1362 (K); Mt. Ayanganna, Pakaraima Mts., Maguire, Bagshaw \& Maguire 40595 (US).

\section{Besleria L.; Sp. Pl. 619. 1753.}

Terrestrial or sometimes epiphytic shrubs, subshrubş, or herbs of very diverse size, up to $6 \mathrm{~m}$ high. Stems erect, ascending, or sometimes creeping or climbing, usually branched, woody or herbaceous, sometimes, with spreading adventitious roots; twigs mostly sappy. Leaves

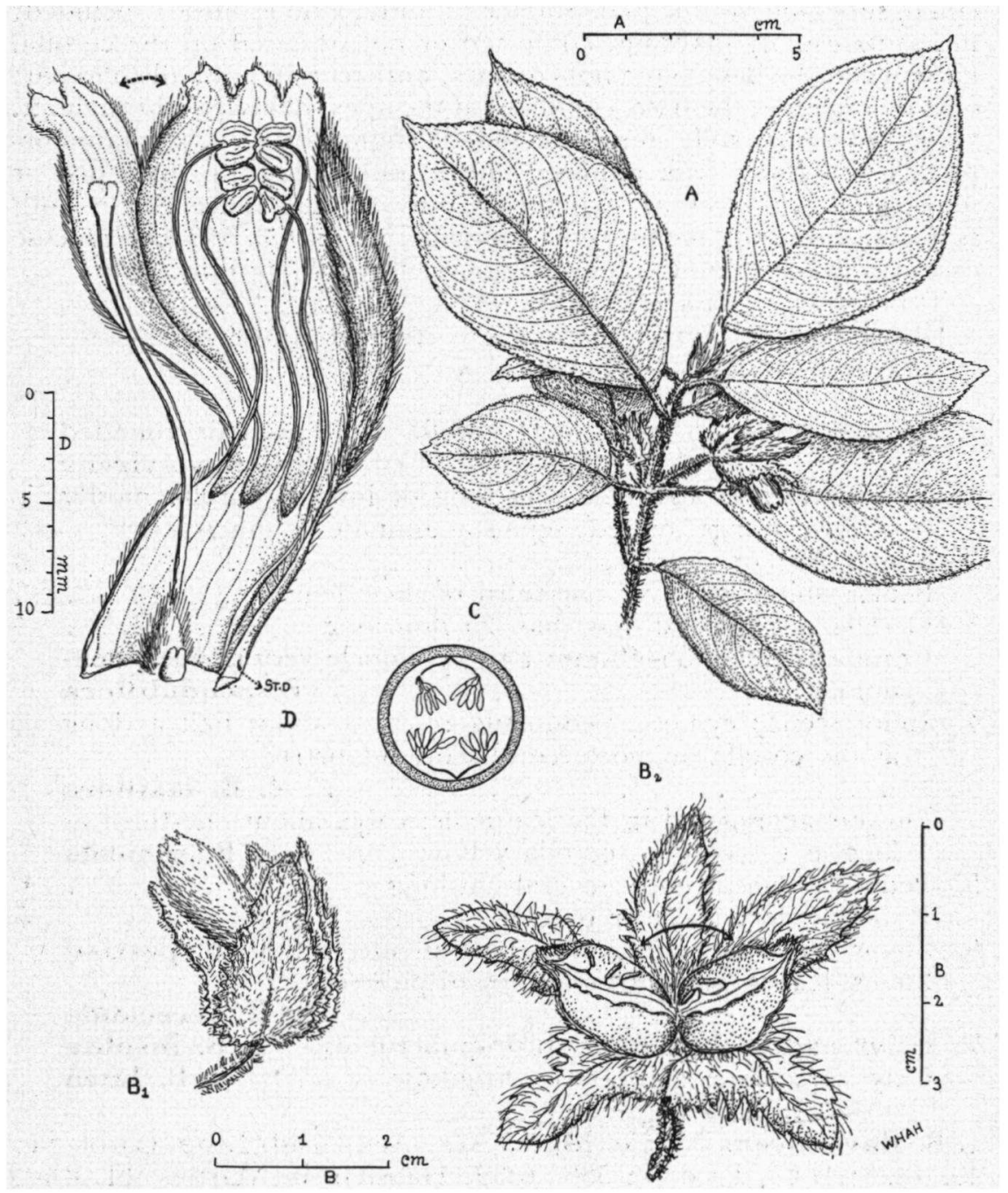

Fig. 9, Alloplectus savannarum: A. branch (Maguire a.o. 40595); $\mathbf{B}_{\mathbf{1}}$. flower; $\mathbf{B}_{2}$. open capsule; C. diagrammatic transverse section of ovary; $D$. opened corolla (B-D: Maguire \& Fanshawe 23127). 
opposite, those of a pair equal, subequal, or sometimes unequal, petiolate; blade usually elliptic. Flowers ebracteate, commonly borne in axillary pedunculate or sessile umbels, rarely solitary. Calyx coloured or green, campanulate or urceolate, rarely cylindric, 5toothed or mostly 5-parted almost to the base; sepals imbricate in aestivation, rounded to acuminate, mostly entire. Corolla yellow, orange, red, or white, often cylindric, erect, oblique, or horizontal in the calyx, spurred at the base or not, usually ventricose; limb sometimes oblique on the tube, actinomorphic or zygomorphic; lobes 5 , equal, subequal, or unequal. Stamens 4, didynamous, mostly included; filaments usually glabrous, contorted or not, inserted on the corolla, flattened at the base, often not connate; anthers reniform, all coherent; cells divergent at the base, confluent at the apex, dehiscent throughout. Ovary superior; style mostly included; stigma bilobed, stomatomorphic, or capitate. Disk annular, entire, rarely dorsally thickened or semi-annular. Placentae ovuliferous only on the inner or on both surfaces. Fruit an indehiscent fleshy berry, with a thick verrucose exocarp. Seeds shining, often obliquely ellipsoid, sprirally striate.

Type species: Besleria lutea L.

Distribution: About 170 species in tropical America.

Key to the species:

1. Flowers small, $5 \mathrm{~mm}$ long; corolla white; sepals rounded 1. B. flavo-virens Flowers larger, about $20 \mathrm{~mm}$ long or longer; corolla mostly yellow, orange, or red; sepals acuminate or mucronate . . 2

2. Sepals free or nearly so . . . . . . . . . . . . . 3

Sepals united for over one-third of their length . . . . . 5

3. Corolla not spurred, less than $25 \mathrm{~mm}$ long . . . . . . . . 4

Corolla spurred, 35-40 mm long; peduncle very slender, pendulous . . . . . . . . . . 7. B. pendulifiora

4. Inflorescence cymose, pedunculate; calyx about half as long as the corolla or more; corolla orange or red

4. B. laxiflora

Flowers aggregated in the leaf axils; calyx about one-third as long as the corolla; corolla yellow . . . 8. B. saxicola

5. Peduncle conspicuous, several $\mathrm{cm}$ long . . . . . . . 6

Peduncle very short or obsolete . . . . . . . . . . 7

6. Stems, leaves beneath, and sepals hirsute . . 6. B. patrisii Stems, leaves, and sepals appressed-pubescent

7. Calyx narrowly cylindric hirto-puberulous 2 B insolita Calyx urceolate, glabrate or strigillose . . . 5. B. lutea

1. B. Alavo-virens Nees et Mart., Nov. Act. Acad. Leop. Carol. 11 : 49. 1823; D.G., Prod. 7: 538. 1839; Hanstein in Martius, Fl. Bras. 8 (1): 400, t. 65. f. 6. 1864; Linnaea 34: 322. 1865; Fritsch in Engl., Bot. Jahrb. 37: 483. 1906; Morton, Contrib. U.S. Nat. Herb. 26: 473. 1939. 
Type: Brazil: Bahia, Ilheos, Felisberta, vic. of São Pedro de Alcantara, von Wied, anno 1816 (BR, 2 sheets, photographs of one: U, US).

Homotypic synonym: Besleria luteo-virens Mart., Nov. Gen. 3: 45. 1829; G. Don, Gen. Syst. 4: 651. 1838.

Leaves of a pair equal. Inflorescence congested-paniculate, pedunculate. Flowers small, numerous, aggregated. Pedicels irregularly branched. Sepals connate at the base, orbicular or nearly so, $1-1 \frac{1}{2} \times$ as long as wide, $2-3 \times 2-2.5 \mathrm{~mm}$, rounded or obscurely emarginate at the apex. Corolla white (bud purple at the apex), about $2 \times$ as long as the calyx, $5 \mathrm{~mm}$ long, at the base and at the throat $2 \mathrm{~mm}$ wide, not spurred or gibbous at the base, contracted in the throat, dorsally bag-shaped.

Terrestrial subshrub or herb, $0.30-2.00 \mathrm{~m}$ high. Stems erect or ascending, sometimes with spreading adventitious roots, puberulous at the apex, glabrescent. Leaves: petiole practically glabrous, $1.5-6 \mathrm{~cm}$ long; blade dark green above, paler beneath, oblong-lanceolate, variable, 2.5-3.5.(mostly about 3 ) $\times$ as long as wide, $12.5-32 \times 5-12 \mathrm{~cm}$, papyraceous when dry, acuminate at the apex, cuneate at the base, more or less serrate, glabrous above, appressed-pubescent beneath
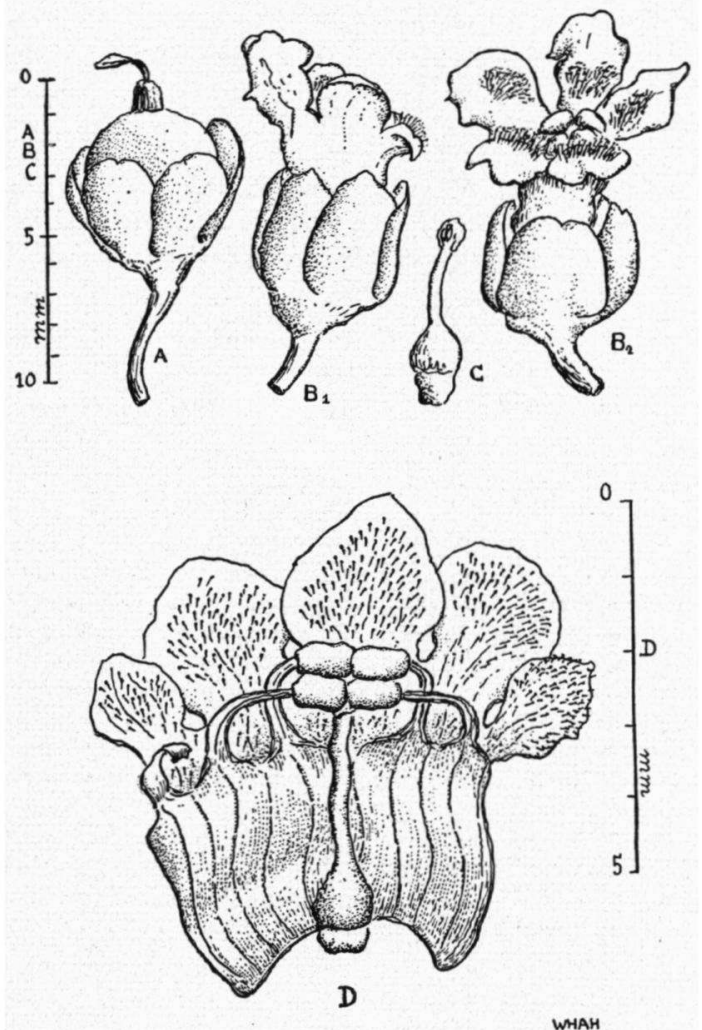

Fig. 10. Besleria flavo-virens (Lanjouw \& Lindeman 2373): A. immature fruit; B. flower; C. pistil; D. opened corolla with pistil. 
especially on the midrib and veins. Inflorescence: peduncle nearly glabrous, 5-25 $\mathrm{mm}$ long, with irregular branchings. Pedicels puberulous when young, glabrescent, 3-10 mm long. Sepals green(?), entire, outside puberulous when young and glabrescent, inside glabrous. Corolla glabrous outside, inside with an annular area of glandular hairs from the insertion of the stamens to the lower part of the lobes; tube 3.5-4 mm long; limb spreading, slightly oblique on the tube, 3-4 mm wide; lobes subequal, rounded, entire, 2 dorsal ones smaller than the others, $1 \mathrm{~mm}$, 3 ventral ones $1.5 \mathrm{~mm}$ in diam. Stamens hardly included; filaments glabrous, not contorted, free from each other, inserted on the middle of the corolla tube. Staminode $1 \mathrm{~mm}$ long, glabrous, with a sterile anther. Ovary broadly ovoid, $0.7 \mathrm{~mm}$ long and wide, glabrous; style glabrous, $3 \mathrm{~mm}$ long; stigma capitate. Disk annular, $0.5 \mathrm{~mm}$ high, entire, glabrous. Placentae ovuliferous on both surfaces. Berry red (teste Maguire), globose, 4-6 mm in diam., shortly apiculate. Seeds reddish-brown, obliquely ellipsoid, $1 \frac{1}{2}-2 \times$ as long as wide, $0.25-0.3 \times 0.15-0.2 \mathrm{~mm}$.

Distribution: Suriname, French Guiana, Brazil (Bahia).

Ecology: In rainforests, at middle elevations.

Suriname: Tafelberg, Maguire 24539 (NY, A, U, US); Nassau Mts., Lanjouw \& Lindeman 2373 (U).

French Gutana: Leprieur, anno 1838 (P, U), anno 1839 (G); Martin 276 (BM), s.n. (BM); Martin, herb. Rudge s.n. (BM); L. C. Richard s.n. (P); vic. of Oyac R., Aroura trib., Aublet s.n. (BM), identified by Aublet as B. cristata.

2. B. insolita Morton, Phytologia 1: 153. 1935; Contrib. U.S. Nat. Herb. 26: 458. 1939; Lemée, Fl. Guian. fr. 3 : 460. 1953. Fig. 12I, p. 372

Type: French Guiana: Cayenne, Martin s.n. (K, photographs: F, US).

Leaves of a pair equal or subequal, long-petiolate. Flowers fasciculate. Calyx yellow, cylindric, about $2 \frac{1}{2} \times$ as long as wide, $10-19 \times$ 4-7 mm, hirto-puberulous outside, glabrous inside; sepals highly connate; tube 7-13 mm long; lobes half as long as the tube, erect, lanceolate, about $3 \times$ as long as wide, $3-6 \times 1-2 \mathrm{~mm}$, mucronate, entire. Corolla creamy or pale yellow, erect in the calyx, 1.25-1.3 $\times$ as long as the calyx, 16-20 mm long, not spurred, hardly ventricose, glabrous outside; limb erect; lobes small.

Terrestrial herb or subshrub, $0.50-1.25 \mathrm{~m}$ high. Stems sappy, at least near the apex, erect, unbranched, terete, near the apex hirto-strigose, glabrescent. Leaves: petiole strigillose, 2.5-11.5 (mostly 4-9) cm long; blade dark green above, paler beneath, elliptic or oblong-elliptic, $2-3$ (mostly $2 \frac{1}{2}-3$ ) $\times$ as long as wide, 9.5-27 $\times$ 4-11 cm, papyraceous when dry, shortly acuminate at the apex, cuneate at the base, serrate, except at the base, sparsely strigose above, strigillose beneath, especially on the midrib and veins. Inflorescence: pedicels hirto-puberulous, 5-20 mm long. Corolla at the base and at the throat $3-4 \mathrm{~mm}$ wide, inside with a ring of glandular hairs in the throat; tube cylindric, 14-18 $\mathrm{mm}$ long; lobes subequal, rounded, entire, $2 \mathrm{~mm}$ in diam. Stamens included; filaments glabrous, not contorted, inserted on the corolla at $4-5 \mathrm{~mm}$ from the base. Staminode glabrous, $2 \mathrm{~mm}$ long, inserted on the corolla at the same level as the stamens, with a sterile anther. Ovary glabrous, ovoid, $5 \times 4 \mathrm{~mm}$; style about as long as the stamens, glabrous; stigma bilobed. Disk annular, entire or sinuate, glabrous, $1.5 \mathrm{~mm}$ wide. Placentae ovuliferous on both surfaces. Berry red (teste L. C. Richard), included in the calyx, subglobose, $7 \times 6 \mathrm{~mm}$. Seeds pale brown, obliquely ellipsoid, about $1 \frac{1}{4}$ as long as wide, $0.25 \times 0.2 \mathrm{~mm}$.

Distribution: Guiana.

Ecology: In rainforests; alt. 0-300 m.

British Gulana: Holmia, Potaro R., Bartlett 8743 (K, NY, paratypes). French Guiana: Mt. Kaw, Cowan 38776 (U); Cayenne, Martin s.n. (K, holotype), 
s.n. (P, U); Martin, herb. Rudge s.n. (BM); Poiteau s.n. (P, G, K, W, paratypes, photograph of $\mathrm{K}$ sheet in US); L. C. Richard s.n. (P); Leprieur, anno 1839 (G). Brazil: Amapá: Serro do Navio, R. Amapari, Cowan 38247 (US), 38453 (US).

3. B. Lanceolata Urb., Symb. Ant. 2: 351. 1901; Morton, Contrib. U.S. Nat. Herb. 26: 427. 1939 (exclusive of synonym). Lectotype: Belanger 193 (G, photographs: U, WAG).

Endemic species of Martinique. In the collection Lamarck at Paris there is one sheet labelled: "French. Guiana". As it is the only sheet from Guiana the present author supposes the labels have been mixed up. In the above key it runs to $B$. saxicola from which it can be distinguished by the narrower coriaceous leaves.

4. B. laxiflora Benth., Lond. Journ. Bot. 5: 361. 1846; Hanstein, Linnaea 34: 324. 1865; Morton, Phytologia 1: 151. 1935; Field Mus. Publ. Bot. 18: 1152. 1938; Contrib. U.S. Nat. Herb. 26: 453. 1939; Fieldiana 28: 1092. 1957.

Fig. 11, p. 370

Type: British Guiana: Schomburgk 205 (K, photograph: US). Heterotypic synonyms: $B$. chiapensis Brandegee, Univ. Calif. Publ. Bot. 6: 64. 1914; 1.c., p. 193. 1915. Type: Mexrco: Chiapas, Finca Mexiquito, Purpus 7003 (UC, holotype, isotypes: BM, F, MO, NY, UC, 2 sheets).

$B$. debilis Rusby, Descr. New S. Amer. Pl. 123. 1920. Type: ColomBIA : Santa Marta, below Valparaiso, H. H. Smith 1399 (NY, holotype, isotypes: $\mathbf{B M}, \mathbf{K}$ ).

B. tenuifolia Rusby, 1.c., p. 124. Type: Colombia: Magdalena, Santa Marta, near the coast at Don Diego, H. H. Smith 2672 (NY, holotype, isotype: $\mathrm{K}$ ).

Leaves of a pair equal or subequal. Inflorescence umbellate or subcymose, 1-10-flowered. Peduncle sparsely strigillose, $15-65 \mathrm{~mm}$ long. Calyx mostly orange or red, sometimes yellow or green, exceedingly variable; sepals almost free, ovate, ovate-lanceolate, or lanceolate, $2-4 \times$ as long as wide, $6-17 \times 2-5 \mathrm{~mm}$, attenuate into a mucro, entire. Corolla red or orange, erect in the calyx, 1.5-3 $\times$ as long as the calyx, 13-23 mm long, not spurred or gibbous at the base, slightly ventricose, glabrous outside; tube cylindric, $11.5-20 \mathrm{~mm}$ long; limb spreading.

Terrestrial shrub or suffrutescent herb, $0.60-3.60 \mathrm{~m}$ (up to $7.50 \mathrm{~m}$, teste von Wedel 709) high. Stems sappy, erect, branched, densely strigose when young, glabrescent. Leaves: petiole sparsely strigillose, $1-6 \mathrm{~cm}$ long; blade dark green above, paler beneath, elliptic or narrowly oblong, $1.5-3.5 \times$ as long as wide, variable, 3-25 × 1-8.5 cm (mostly about 10-20 $\times$ 4-7 cm), membranaceous when dry, acuminate at the apex, cuneate at the base, serrulate, above practically glabrous, sparsely strigillose beneath, especially on the midrib and veins. Inflorescence: pedicels sparsely strigillose, $8-25 \mathrm{~mm}$ long. Sepals glabrous or outside sparsely strigillose. Corolla at the base 3-6.5 $\mathrm{mm}$, at the throat 4-7 mm wide, inside with a ring of hairs near the insertion of the stamens and a second ring of glandular hairs in the throat; lobes subequal, rounded, entire, $1.5-3 \mathrm{~mm}$ in diam. Stamens included; filaments glabrous, contorted or not, free from each other, inserted below the middle of the corolla tube. Staminode glabrous, $1.5-3 \mathrm{~mm}$ long, with a sterile anther. Ovary glabrous, ovoid, 2.5-4 × 2-3 mm; style glabrous; stigma stomatomorphic. Disk annular, entire, glabrous. Placentae ovuliferous on both surfaces. Berry orange or red, globose, shortly apiculate, $10-12 \mathrm{~mm}$ in diam. Seeds reddish-brown, $1-2 \times$ as long as wide, $0.3-0.4 \times 0.2-0.3 \mathrm{~mm}$. 
Distribution: Mexico, Central America, Colombia, Venezuela, Guiana, Brazil (Rio Branco).

Ecology: In dense rainforests on banks of watercourses or on slopes, sometimes in clearings; alt. 0-1400 m.

British Guiana: Schomburgk 205 (K, type); Kanuku Mts., Moku-moku Ck., Takutu trib., A. C. Smith 3555 (NY, F, G, K, MO, S, U, US); Upper Essequibo R., Mataruki R., Meyers 5839 (K); Basin of Shodikar Ck., Essequibo trib., A. C. Smith 2886 (NY, F, G, K, U, US); New R. and Oronoque R. Heads, Beddington $33(\mathrm{~K})$.

SURINAME: Emmaketen, B.W. 5705 (U); Brownsberg, B.W. 645 (U).

French Guiana: Perrottet, anno 1820 (P, G); herb. expos. col. s.n. (P).

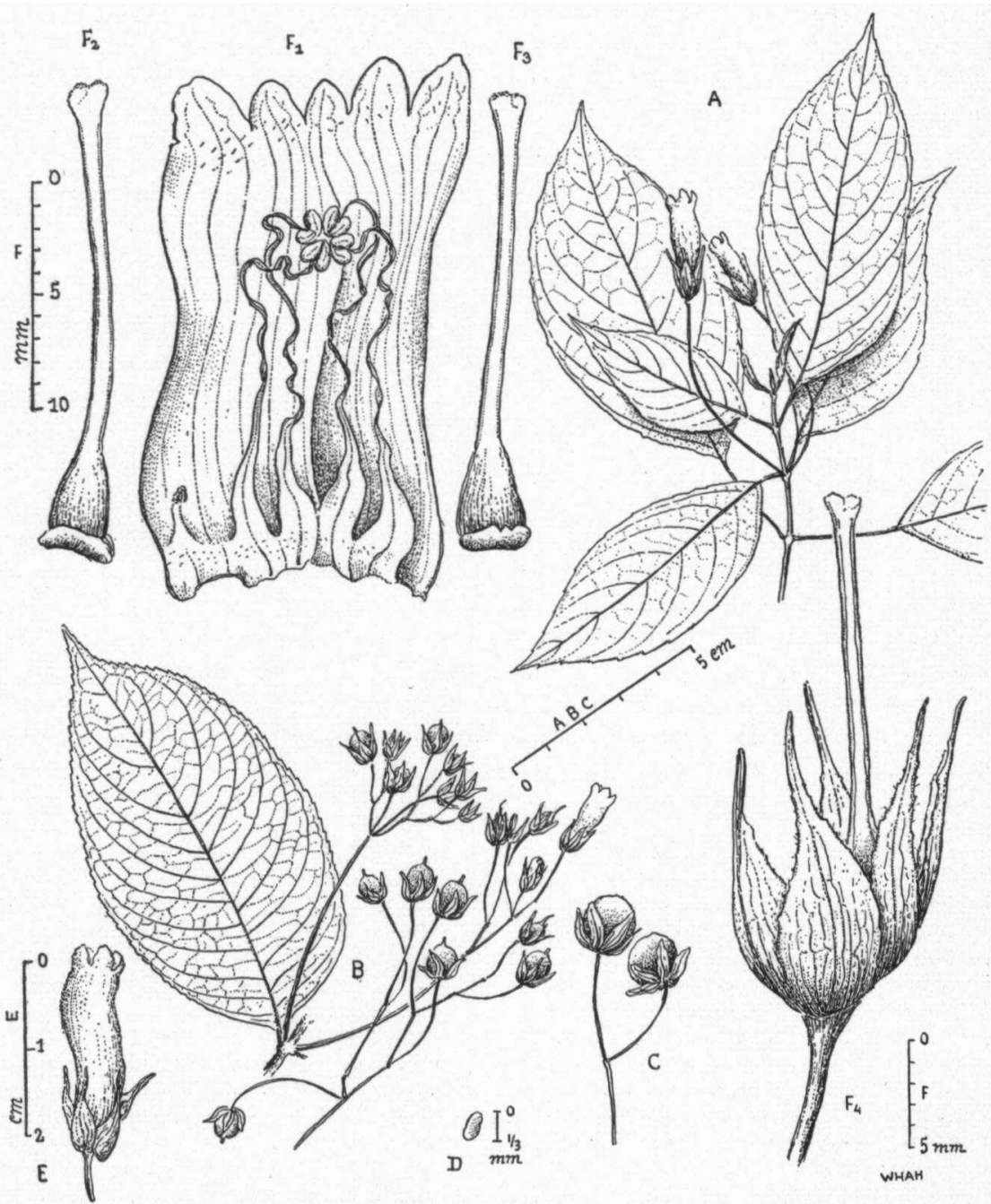

Fig. 11. Besleria laxiflora: A. branch (Karsten s.n.); B. branch with flower and immature fruits (Ule 8459); C. mature fruits; D. seed (C-D: H. H. Smith 2672); E. flower; $F_{1}$. opened corolla; $F_{2}$ and $F_{2}$. pistils; $F_{\mathbf{a}}$. calyx with pistil (E-F, B. W. 645). 
5. B. lutea L., Sp. Pl. 619. 1753; G. Don, Gen. Syst. 4: 651. 1838; D.C., Prod. 7: 538. 1839; Oersted, Centralamer. Gesn. 54, t. 11 . f. 1-10. 1858; Grisebach, Fl. Br. W.I. Isl. 463. 1861 ("1864"); Hanstein, Linnaea 34; 316. 1865; Duss, Fl. Ant. fr. 431. 1897; Urban, Symb. Ant. 2: 347. 1901; op. cit. 9: 268. 1925; in Fedde, Repert. Beih. 5: 46. 1920; Boldingh, Fl. W.I. Eil. 365. 1913; Morton, Contrib. U.S. Nat. Herb. 26: 457. 1939; in Bro. Leon \& Bro. Alain, Fl. Cuba 4: 470. 1957.

Neotype: Burmann, Plant. Amer. Car. Plumier. 1756. t. 49. f. 1, "Besleria pedunculis simplicibus confertis".

Heterotypic synonyms: $B$. berteriana D.C., Prod. 7: 538. 1839. Type: Jamaica: Bertero s.n. (G-DG).

$B$ imrayi Hook., Bot. Mag. 104: t. 6341. 1878. Type: Cult. Hort. Kew., Imray s.n., from Dominica(K).

Shrub or tree $1-7 \mathrm{~m}$ high. Stems erect or rarely scandent, branched, glabrous and shining at maturity. Leaves petiolate or sometimes subsessile; petiole 1-35 (mostly 10-20) mm long; blade dark green above, paler beneath, ovate, elliptic, or oblong, $1 \frac{1}{2}-2 \frac{1}{2} \times$ as long as wide, $5 \frac{1}{2}-28 \times 2-10 \mathrm{~cm}$, papyraceous or chartaceous when dry, acuminate at the apex, cuneate or rounded at the base, serrate or serrulate, glabrous on both sides or strigillose on the midrib and veins beneath. Inflorescence: peduncle very short or usually obsolete. Flowers 1-6 (mostly 3-4) together. Calyx urceolate, $6-10 \mathrm{~mm}$ long and $4-6 \mathrm{~mm}$ wide; sepals connate for two-thirds of their length or more, entire, mucronate, sparsely strigillose outside. Corolla yellow or rarely white, erect in the calyx, not spurred nor gibbous at the base, contracted in the throat, slightly ventricose, glabrous on both sides; lobes small, subequal, rounded, entire, $2-3 \mathrm{~mm}$ in diam. Stamens included; filaments glabrous, free from each other, not contorted, inserted below the middle of the corolla tube. Staminode present. Ovary glabrous, ovoid, $4 \times 2.5 \mathrm{~mm}$; style glabrous; stigma capitate. Disk annular. Placentae ovuliferous on both surfaces. Berry red, globose, 5-8 $\mathrm{mm}$ in diam.

A common West Indian species. In the Paris herbarium there is only one sheet labelled: "French Guiana", collected by L. C. Richard. The correctness of the label is open to doubt. Aublet's specimen (1775, p. 637) is B. verecunda. The specimens mentioned by SchomburGK $(1848$, p. 971$)$ have been destroyed in Berlin. It remains doubtful if this species occurs in Guiana.

6. B. patrisii D.C., Prod. 7 : 538. 1839; Morton, Contrib. U.S. Nat. Herb. 26: 452. 1939; Lemée, Fl. Guian. fr. 3: 459. 1953.

Type: French Guiana:' Patris s.n. (G-DC, 2 sheets, photographs of first: GH, US).

Fig. 12 P, p. 372

Stems erect, hirsute, glabrescent. Leaves opposite or sometimes three together, those of a pair or a whorl equal or subequal; blade hirsute beneath, especially on the midrib and veins. Inflorescence umbellate or subcymose, 1-6-flowered. Peduncle hirsute, $1-3.5 \mathrm{~cm}$ long. Calyx campanulate; sepals oblong-lanceolate, $3-5 \times$ as long as wide, $10-15 \times 2-3 \mathrm{~mm}$, connate to the middle, attenuate into a mucro, entire, hirsute outside, glabrous inside. Corolla red(?), cylindric, erect in the calyx, 1.5-2 $\times$ as long as the calyx, 18-22 mm long, not spurred or gibbous at the base, glabrous outside; lobes small.

Terrestrial suffrutescent(?) herb. Leaves: petiole hirsute, $1-4.5 \mathrm{~cm}$ long; blade dark green above, paler beneath, oblong-elliptic, about $2 \frac{1}{2} \times$ as long as wide, $11-20 \times 3.5-8 \mathrm{~cm}$, papyraceous when dry, shortly acuminate at the apex, cuneate at the base, more or less serrate, sparsely hirsute-above. Inflorescence: pedicels sparsely hirsute, $0.5-3 \mathrm{~cm}$ long. Corolla at the base $4-5 \mathrm{~mm}$, at the throat 5-7 $\mathrm{mm}$ wide, not ventricose, inside with a ring of hairs near the insertion of the stamens 
and a second ring of glandular hairs in the throat; lobes subequal, rounded, obscurely sinuate, about $2 \mathrm{~mm}$ in diam. Stamens included; filaments glabrous, not contorted, free from each other, inserted below the middle of the corolla tube. Staminode glabrous, $2.5 \mathrm{~mm}$ long, with a sterile anther. Ovary glabrous, ovoid, $5 \times 4 \mathrm{~mm}$; style glabrous; stigma bilobed. Disk annular, entire, glabrous. Placentae ovuliferous on both surfaces. Fruit and seeds not seen.

Distribution: French Guiana.

Ecology: No data extant.

French Gutana: Patris s.n. (G-DC, type); Leprieur, anno 1850 (P, U).

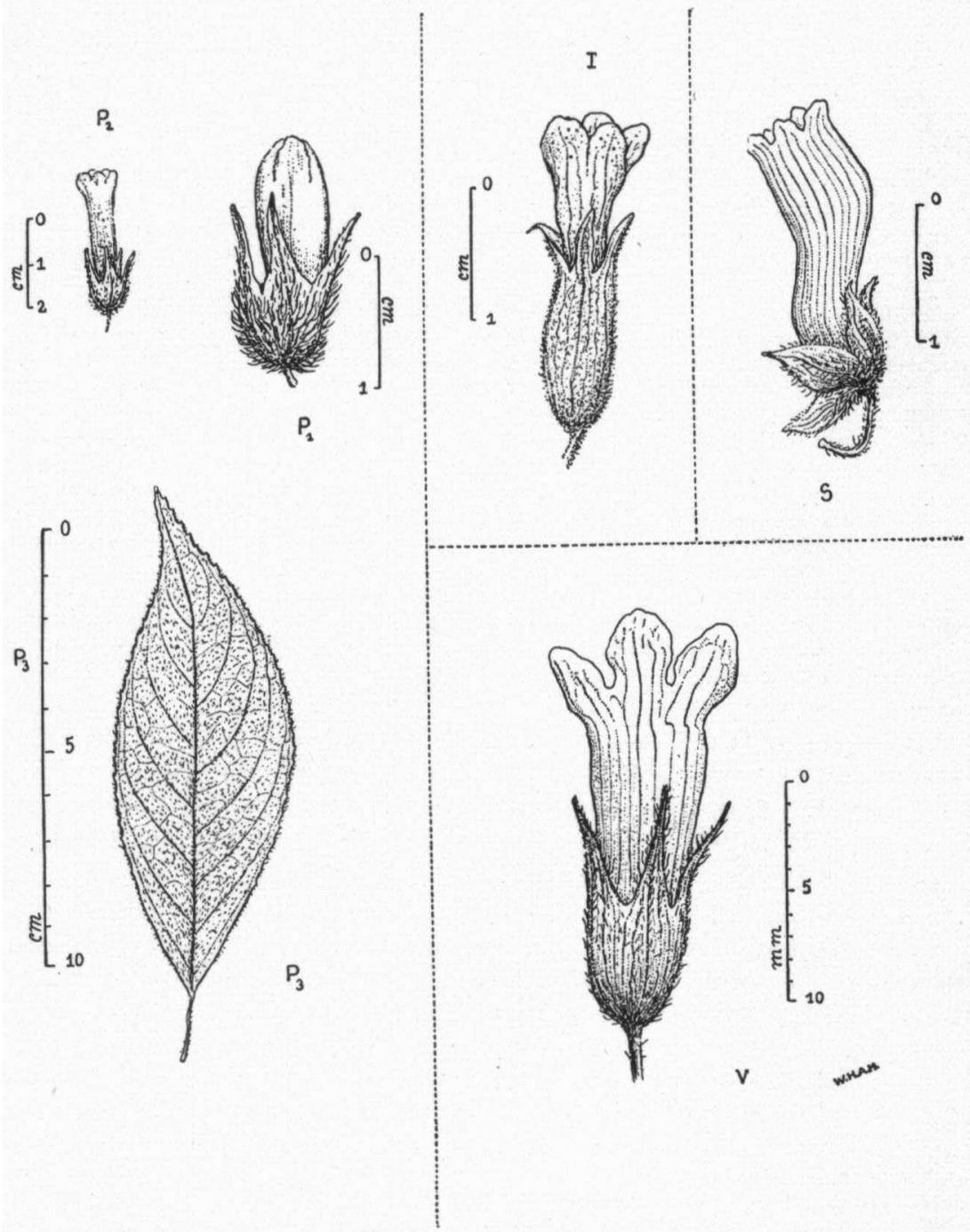

Fig. 12. I. flower of Besleria insolita (Martin, herb. Rudge); P. B. patrisii (Leprieur, anno 1850): 1. flower-bud; 2. flower; 3. leaf; S. flower of $B$. saxicola (Hitchcock 17375); V. flower of $B$. verecunda (Jenman 4156). 
7. B. penduliflora Fritsch in Fedde, Repert. 18: 9. 1922; Morton, Contrib. U.S. Nat. Herb. 26: 458. 1939.

Type: Venezuela: Roraima, Ule 8751 (G, K, L, U, isotypes, fragment in F, photograph of G sheet in US; holotype destroyed in B, photograph in US).

Leaves of a pair equal or unequal; blade lanceolate, dark green and densely strigillose above, grey-green and tomentose beneath. Inflorescence umbellate or subcymose, 1-3-flowered. Peduncle very slender, pendulous, tomentose, 35-65 $\mathrm{mm}$ long. Sepals green(?), connate at the base, ovate-lanceolate, $2-3.5 \times$ as long as wide, $6-7 \times$ 2-3 mm, acuminate at the recurved apex, entire, unequal, the dorsal one narrower than the others, curved around the spur. Corolla yellow, strongly oblique in the calyx, 5-6 $\times$ as long as the calyx, $35-40 \mathrm{~mm}$ long, spurred at the base; limb spreading, about $15 \mathrm{~mm}$ wide.

Terrestrial shrub, 1.50-6.00 m high. Stems woody, erect, branched; twigs tomentose, glabrescent. Leaves: petiole tomentose, 5-20 mm long; blade 2.5-4 (mostly 3.5-4) $\times$ as long as wide, 3.5-13.5 $\times 1-5 \mathrm{~cm}$, papyraceous when dry, acuminate at the apex, cuneate or rounded at the base, remotely denticulate. Inflorescence: pedicels slender, subtomentose, 15-45 mm long. Sepals strigillose outside, puberulous inside. Corolla at the base about $5 \mathrm{~mm}$, at the throat about $10 \mathrm{~mm}$ wide, slightly widened, not at all ventricose, somewhat contracted above the spur but not in the throat, very sparingly pilosulous outside, inside with a ring of glandular hairs in the throat; tube about $30 \mathrm{~mm}$ long; spur rounded, about $3 \mathrm{~mm}$ long; lobes subequal, rounded, entire, about $4 \mathrm{~mm}$ in diam. Stamens included or slightly exserted; filaments glabrous, not contorted, free from each other, inserted on the corolla tube at $8 \mathrm{~mm}$ from the base. Staminode glabrous, $5 \mathrm{~mm}$ long, with a sterile anther. Ovary densely strigillose, ovoid, $5 \times 3.5 \mathrm{~mm}$; style densely strigillose; stigma bilobed (?). Disk annular, entire, glabrous. Placentae ovuliferous on both surfaces. Berry globose, strigillose, shortly apiculate, $10-11 \mathrm{~mm}$ in diam. Seeds reddish-brown, ellipsoid, $1.5-3 \times$ as long as wide, $0.6-1 \times 0.3-0.5 \mathrm{~mm}$.

Distribution: Guayana Highlands.

Ecology: In montane rainforests on sandstone(?), at higher elevations.

Brmish Gulana: Roraima, im Thurn 287 (K, BM).

8. B. saxicola Morton, Phytologia 1: 151. 1935; Contrib. U.S. Nat. Herb. 26: 431. 1939.

Fig. 12 S, p. 372

Type: British Guiana: Potaro R., Tumatumari, Hitchcock 17375 (US, holotype, isotype: NY).

Stems hirsute at the apex, glabrescent. Leaves of a pair equal or subequal, long-petiolate; petiole hirsute; blade sparsely hirsute on both sides. Flowers fasciculate, numerous. Pedicels short, hirsute, 5-10 mm long. Sepals yellow, free, subequal, ovate or ovate-lanceolate, $2-3.5 \times$ as long as wide, $6-8 \times 2-3 \mathrm{~mm}$, subulate-acuminate, entire, hirsute outside, long-ciliate, glabrous inside. Corolla yellow, oblique in the calyx, about $3 \times$ as long as the calyx, $18-20 \mathrm{~mm}$ long, gibbous at the base, glabrous outside; limb hardly spreading.

Terrestrial shrub, subshrub, or herb, $0.30-1.80 \mathrm{~m}$ high. Stems mostly unbranched, terete. Leaves: petiole 3-12 cm long; blade dark green above, paler beneath, obliquely elliptic, 2-2.5 $\times$ as long as wide, $20-30 \times 7-13 \mathrm{~cm}$, membranaceous when dry, short-acuminate at the apex, cuneate at the base, remotely sharpserrulate, hirsute on the midrib and veins beneath. Corolla at the base $3-5 \mathrm{~mm}$, 
at the throat 5-6 mm wide, hardly contracted above the gibbosity, more conspicuously so in the throat, ventrally somewhat ventricose, inside with a ring of glandular hairs in the throat; tube 17-18 $\mathrm{mm}$ long; lobes subequal, broadly rounded, 1-1.3 $\times$ as wide as long, $1.5-2 \times 1.5-2 \mathrm{~mm}$, entire. Stamens included; filaments glabrous, not contorted, free from each other, inserted below the middle of the corolla tube. Staminode glabrous, $3 \mathrm{~mm}$ long, with a sterile anther. Ovary glabrous, conic, about as long as wide, 2-3 mm long; style puberulous; stigma bilobed. Disk semiannular, dorsal, entire, glabrous. Placentae ovuliferous on both surfaces. Berry yellow, globose, shortly apiculate, $8-10 \mathrm{~mm}$ in diam. Seeds dark brown, obliquely ellipsoid, $1-2 \times$ as long as wide, $0.25-0.3 \times 0.15-0.25 \mathrm{~mm}$.

Distribution: British Guiana.

Ecology: In rainforests, on moist, often rocky soil; alt. 0-80 m. British Guiana: Winiporo Ck., F.D. 3024 (K); Essequibo R., Moraballi Ck., near Bartica, Sandwith 58 (K, NY, paratypes); Potaro R., Abraham 345 (K, $\mathrm{U}$, paratypes); ibid., Tumatumari, Gleason 420 (NY, GH, US, paratypes); ibid., Hitchcock 17375 (US, holotype, NY, isotype); Kurupung R., Kumara, Altson $311(\mathrm{~K}, \mathrm{NY})$.

9. B. verecunda Morton, Phytologia 1: 151. 1935; Contrib. U.S. Nat. Herb. 26: 453. 1939.

Fig. 12 V, p. 372

Type: British Guiana: Upper Demerara R., Jenman 4156 (Morton errore 5156) (US, holotype, isotypes: $\mathrm{K}, 2$ sheets, NY, US).

Leaves of a pair equal or subequal. Inflorescence umbellate or subcymose, 1-5-flowered. Peduncle strigillose, $1-6 \mathrm{~cm}$ long. Calyx yellow or pale green, campanulate, $10-15 \times 4-7 \mathrm{~mm}$; sepals united for 5-6 mm, lanceolate, 3-5 $\times$ as long as wide, 3-5 $\mathrm{mm}$ wide, subulateacuminate, entire, glabrous or outside with scattered hairs. Corolla red, erect in the calyx, 1.5-2 $\times$ as long as the calyx, 17-25 mm long, not spurred or gibbous at the base, glabrous outside; limb small, spreading.

Terrestrial shrub or subshrub, 0.90-1.50 m high. Stems sappy, erect, branched or not, terete, strigose at the apex, glabrescent. Leaves: petiole sparsely strigillose, 1-7 cm long; blade dark green above, paler beneath, elliptic or oblong-elliptic, 2t-3 (mostly $2 \frac{1}{2}$ ) $\times$ as long as wide, 5-22 $\times 1.5-10 \mathrm{~cm}$, papyraceous when dry, acuminate at the apex, cuneate at the base, serrate or repand-serrate; with scattered hairs above, strigose beneath, especially on the midrib and veins. Inflorescence: pedicels sparsely strigillose, 5-30 $\mathrm{mm}$ long. Gorolla at the base $3-5 \mathrm{~mm}$, at the throat 4-8 $\mathrm{mm}$ wide, slightly contracted in the throat, hardly ventricose, inside with some hairs near the insertion of the stamens and a ring of glandular hairs in the throat; tube cylindric, 15-22 mm long; lobes subequal, rounded, entire, 2-3 $\mathrm{mm}$ in diam. Stamens included; filaments glabrous, contorted or not, free from each other, inserted below the middle of the corolla tube. Staminode glabrous, $2-3 \mathrm{~mm}$ long, with a sterile anther. Ovary glabrous, ovoid, 3-4 $\times 2.5-3 \mathrm{~mm}$; style glabrous; stigma bilobed. Disk annular, entire, glabrous. Placentae ovuliferous on both surfaces. Berry globose, apiculate at the apex, 10-12 $\mathrm{mm}$ in diam. Seeds red (teste Morton) or dark brown, ellipsoid, $1-2 \times$. as long as wide, $0.25-0.3 \times$ $0.15-0.25 \mathrm{~mm}$.

Distribution: Guianas.

Ecology: In rainforests; alt. 0-200 m.

British Gutana: Demerara R., Sibaruni Ck., F.D. 6334 (K, NY); Upper Demerara R., Jenman 4156 (US, holotype, isotypes: $K, 2$ sheet, NY, US).

SURINAME: Upper Litanie R., Versteeg 342 (U), 354 (U).

French Guiana: Galibi Gk., Aublet s.n. (BM), identified as B. lutea. 
3. Chrysothemis Dcne., Rev. Hortic. Sér. 3. 3: 242.1849.

Terrestrial herbs with tubers, $0.05-2.00 \mathrm{~m}$ high. Stems succulent, erect, mostly unbranched, usually with spreading adventitious roots at the base. Leaves opposite, those of a pair equal or subequal, petiolate; blade dark green above, paler green or purple beneath, usually oblong-elliptic, acuminate at the apex, crenate-serrate or nearly so. Inflorescence axillary, umbellate, much shorter than the leaves, several-flowered. Peduncle solitary, with some small bracts which are leafy, lanceolate or nearly so, and puberulous on both sides. Calyx red (Guianan spp.), green, or yellow, campanulate or infundibuliform, wide in proportion to the corolla; sepals united for most of their length. Corolla yellow or orange, red-spotted or -lined on the limb and in the throat or only in the throat, nearly trumpet-shaped, erect in the calyx, $1 \frac{1}{1}-2 \frac{1}{2} \times$ as long as the calyx, not spurred, not or hardly gibbous at the base, not ventricose, glabrous or hairy outside, inside with a ring of hairs near the insertion of the stamens and glandular hairs in the throat; tube cylindric; limb spreading, horizontal on the tube; lobes 5 , subequal, rounded, entire. Stamens 4, didynamous, included; filaments glabrous, contorted or not, all connate at the base, inserted on the base of the corolla; anthers orbicular, coherent or not; cells parallel, discrete, dehiscent throughout by a longitudinal split. Staminode none. Ovary superior, pubescent, ovoid; style mostly glabrous, about as long as the stamens or shorter; stigma bilobed. Disk gland one (Guianan spp.), dorsal, mostly bilobed. Placentae ovuliferous on the inner surface only. Fruit a capsule, included in the calyx, globose, bivalved, obtuse at the apex. Seeds dark brown, shining, ellipsoid, obliquely striate, mostly about $2 \times$ as long as wide, apiculate at the base.

Type species: $C$. pulchella (Donn ex Sims) Dcne.

Distribution: 6 species in the West Indies, Central and northern South America.

Key to the species:

1. Calyx infundibuliform or campanulate, not angled, serrate at the margin or with 5 broadly triangular lobes which are up to about half as long as wide

Calyx campanulate, 5-angled, 5-lobed, lobes about as long as wide, acuminate ....... 1. C. pulchella

2. Leafblades not decurrent into the petiole; calyx serrate or obscurely 5-lobed . . . . . . . 2. C. rupestris

Leafblades decurrent into the petiole; calyx 5-lobed, lobes broadly triangular, about half as long as wide

\section{G. villosa}

1. C. pulchella (Donn ex Sims) Dcne,, Rev. Hortic. Sér. 3. 3: 242. 1849; op. cit. 4: 382. 1850; H. E. Moore, African Violets, Gloxinias, and their relatives 192, 272. 1957.

Basionym: Besleria pulchella Donn [Hort. Cantabr. 4th ed. 140. 1807, (nomen nudum)] ex Sims, Bot. Mag. 28: t. 1146. 1808 (descr. 
et icon.); Loddiges, Bot. Cab. 11: t. 1028. 1825; D.C., Prod. 7: 539. 1839.

Type: Cult., Woodford, Springwell, Hertfordshire, Aug. 1808 (BM). Homotypic synonyms: Tussacia pulchella (Donn ex Sims) Rchb. ex Walpers, Repert. 6: 740. 1847 (see also Reichenbach, Icon. Bot. Exot. 1: 28, t. 41. 1827); Oersted, Centralamer. Gesn. 39, t. 10. f. 14-19. 1858; Grisebach, Fl. Br. W.I. Isl. 463. 1861 ("1864"); Hanstein, Linnaea 34: 337. 1865; Duss, Fl. Ant. fr. 431. 1897; Urban, Symb. Ant. 2: 355. 1901; Morton in Fl. Trin. and Tob. 2 (5): 303. 1955.

Episcia pulchella (Donn ex Sims) Mart. ex G. Don, Gen. Syst. 4: 656. 1838 (see also Martius, Nov. Gen. 3: 45. 1829); Hartinger, Parad. Vindob. 1: 39, t. 66. 1860 ("1848-1860").

Skiophila pulchella (Donn ex Sims) Hanst., Linnaea 26: 207. 1853. Heterotypic synonyms: Chrysothemis aurantiaca Dcne., Rev. Hortic. Sér. 3. 4: 381. 1850. Lectotype: Cult. Hort. Paris, Aug. 1850 (P). Tussacia woodsoni Morton, Ann. Mo. Bot. Gard. 26: 308. 1939. Type: Panama: prov. Chiriquí, between R. Ghiriquí and Remedios, Woodson, Allen \& Seibert 1195 (US, holotype, isotypes: NY, US, photographs of NY sheet in U and WAG).

Leafblade decurrent into the petiole. Calyx orange or red, campanulate, 5-angled, 10-18 mm long, 5-11 mm wide, puberulous or villose outside, especially below, glabrous or minutely puberulous inside; lobes acuminate, dentate. Corolla with red longitudinal lines on the limb and in the throat, $1 \frac{1}{2}-2 \frac{1}{2}$ (mostly about 2) $\times$ as long as the calyx, 16-30 mm long, outside more or less white-sericeous above, glabrous below.

Plant 10-90 cm high. Stems green, sometimes partially purple or with some short red lines, puberulous, often glabrescent. Leaves: blade elliptic, ovate, or oblongovate, variable, $1 \frac{1}{2}-3 \frac{1}{2}$ (mostly about 2 ) $\times$ as long as wide, up to $30 \times 12 \mathrm{~cm}$, membranaceous or papyraceous when dry, sparsely strigillose to villose above, puberulous beneath, especially on the midrib and veins. Inflorescence: peduncle puberulous, 1-5 cm long. Bracts oblong, lanceolate, or sometimes linear, 2-10 (mostly 3-5) $\times$ as long as wide, 4-15 $\times 1-6 \mathrm{~mm}$, acuminate, entire or obscurely serrate, puberulous on both sides. Pedicels puberulous, $1.5-2.5 \mathrm{~cm}$ long. Corolla somewhat gibbous at the base, slightly contracted above the gibbosity; limb 10-15 mm wide; lobes 3-8 $\mathrm{mm}$ in diam. Anthers all coherent or free. Ovary 2-4 $\times 2-3 \mathrm{~mm}$; style about as long as the stamens, glabrous. Disk gland bilobed, rarely entire or several-lobed. Capsule sparsely pubescent, $6 \mathrm{~mm}$ in diam.

Distribution: West Indies, Panama, Colombia, Venezuela, British Guiana, Suriname, and Brazil (Amazonas).

Ecology: On wet rocks in rainforests; alt. 0-300 m.

British Guiana: Pakaraima Mts., Wenamu R., Davenport $8(\mathrm{~K})$; Pomeroon Distr., Moruka R., de la Gruz 4593 (NY, F, GH, MO, PH, US); Great Falls, Demerara R., Jenman 3990 (K, NY, U); spontaneous in Bot. Garden, Jenman 5608 (K); Drake, comm. Campbell, June 1871 (K).

SURINAME: near Avanavero Falls, Kabalebo R., Corantijn R., B.W. 4602 (U); ibid., Florschütz 2213 (U); ibid., Pulle 437 (U); Paramaribo, plant. Combé, Pulle H 13 (U).

2. Chrysothemis rupestris (Benth.) Lwbg., p. 336.

Figs. 2, p. 337; 3 R, p. 339

Basionym: Tussacia rupestris Benth., Lond. Journ. Bot. 5: 363. 
1846; Schomburgk, Reisen Br. Guian. 3: 972. 1848; Hanstein, Linnaea 34: 339. 1865; Morton, Bull. Torr. Bot. Gl. 75: 566. 1948.

Type: British Guiana: Kanuku Mts., Schomburgk s.n. (K, holotype, photographs: U, WAG).

Leafblades not decurrent into the petiole. Calyx orange or red, infundibuliform, 10-15 $\mathrm{mm}$ long, at the margin 6-12 $\mathrm{mm}$ wide, serrate or obscurely 5-lobed, puberulous outside, glabrous inside. Corolla $1 \frac{1}{2}-2 \frac{1}{2}$ (mostly 2) $\times$ as long as the calyx, 18-25 mm long, outside villose above, glabrous below.

Plant 5-200 cm high. Stems green or partially purple, sometimes with short red lines, puberulous at the apex, glabrescent. Leaves: petiole puberulous, $0.5-5 \mathrm{~cm}$ long; blade ovate or oblong-ovate, $1 \frac{1}{2}-2 \frac{1}{2} \times$ as long as wide, of very variable size, up to $24 \times 14.5 \mathrm{~cm}$, membranaceous or papyraceous when dry, cuneate or rounded at the base, sparsely strigillose above, puberulous beneath, especially on the midrib and veins. Inflorescence: peduncle puberulous, $1-6 \mathrm{~cm}$ long. Bracts linear, about $10 \times$ as long as wide, 5-12 $\times 0.2-1 \mathrm{~mm}$, acuminate, entire, puberulous on both sides. Pedicels puberulous, $0.5-2.5 \mathrm{~cm}$ long. Corolla red-lined or -spotted, obscurely gibbous at the base, slightly contracted above the gibbosity; limb $10-15 \mathrm{~mm}$ wide; lobes 4-5 $\mathrm{mm}$ in diam. Anthers coherent in pairs. Ovary $2-4 \times 2-3 \mathrm{~mm}$; style mostly glabrous. Disk gland large, almost as long as the ovary, bilobed, rarely several-lobed. Capsule pubescent, 6-7 $\mathrm{mm}$ in diam.

Distribution: British Guiana and Suriname.

Ecology: On wet, often granitic rocks among shrubs or in rainforests; alt. $0-400 \mathrm{~m}$.

British Guiana: Kanuku Mts., Schomburgk s.n. (K, holotype); Appun s.n., rec. July $1866(\mathbf{K})$.

Suriname: Corantijn R., Wonotobo Falls, Rombouts 119 (U); Wilhelmina Mts., on islet in rapid, B.W. 7226 (U); Tafelberg, Maguire 24762 (NY, U, US); Upper Gran Rio, Hulk 243 (U); ibid., Tresling 470 (U); Upper Tapanahoni R., near Mt. Teboe, Versteeg 760 (U); base of Voltzberg, Schulz 7611 (U); between Saramacca and Suriname Rs., between Pakka Pakka and Ebbatop, Florschütz 1326 (U); near fall in trib. ck. of Kleine Saramacca R., Saramacca R., Florschütz 1400 (U).

3. C. villosa (Benth.) Lwbg., p. 338. Fig. 3 V, p. 339

Basionym: Tussacia villosa Benth., Lond. Journ. Bot. 5: 363. 1846; Schomburgk, Reisen Br. Guian. 3: 972. 1848; Hanstein, Linnaea 34: 338.1865.

Type: BRITISH Guiana: Kanuku Mts., Schomburgk s.n. (K, holotype, photographs: U, WAG).

Leafblades shortly decurrent into the petiole. Calyx red, pale green at the base, campanulate-infundibuliform, 10-17 $\mathrm{mm}$ long, at the limb 9-14 $\mathrm{mm}$ wide, 5-lobed; lobes broadly triangular, about half as long as wide, $2-4 \times 4-8 \mathrm{~mm}$, acute or shortly acuminate, dentate, puberulous outside, glabrous inside. Corolla $1 \frac{3}{4}-2 \frac{1}{2} \times$ as long as the calyx, 25-30 mm long, outside villose above, glabrous below.

Plant 5-40 cm high. Stems green, sometimes with short red lines, puberulous. Leaves: petiole puberulous, $1-3.5 \mathrm{~cm}$ long; blade ovate or oblong-ovate, 1-2 (mostly about $1 \frac{1}{2}$ ) $\times$ as long as wide, 9-24.5 $\times 4.5-14.5 \mathrm{~cm}$, papyraceous when dry, strigillose above, puberulous beneath. Inflorescence: peduncle puberulous, $1-6 \mathrm{~cm}$ long. Bracts linear, $5-20 \times$ as long as wide, $6-15 \times 0.5-2 \mathrm{~mm}$, acuminate, entire, puberulous on both sides. Pedicels puberulous, $0.5-2.5 \mathrm{~cm}$ long. Corolla lobes deep red inside or red-spotted and -lined in the throat, gibbous at the base, 
slightly contracted above the gibbosity; limb $10-13 \mathrm{~mm}$ wide; lobes $4-6 \mathrm{~mm}$ in diam. Anthers coherent in pairs. Ovary 2-4 $\times 2-3 \mathrm{~mm}$; style glabrous. Disk gland large, bilobed. Capsule pubescent, $6-8 \mathrm{~mm}$ in diam.

Distribution: British Guiana.

Ecology: On wet granitic(?) rocks, in rainforests, along watercourses, at middle elevations.

BrtTsh Gutana: Kanuku Mts., Schomburgk s.n. (K, holotype); ibid., in drainage of Moku-moku Ck., Takutu trib., A. C. Smith 3579 (NY, F, G, K, MO, U, US); Komo Gk., Takutu R., F.D.-W.B. 569 (K), 635 (NY).

\section{Codonanthe (Mart.) Hanst., Linnaea 26: 199, 209. 1853.}

Epiphytic or sometimes terrestrial vines, shrubs, subshrubs, or herbs, mostly on arboreal ants' nests; when creeping, climbing, or pendant, $0.15-2.00 \mathrm{~m}$ long, when erect $0.10-2.10 \mathrm{~m}$ high. Stems branched or not, usually with nodigenous adventitious roots, sarmentose, often woody at the base. Leaves opposite, those of a pair equal, mostly rather short-petiolate; blade oblong-ovate, elliptic, or oblongelliptic, usually thick and fleshy, entire or serrulate. Flowers axillary, one to several, mostly short-pedicellate. Bracts none. Sepals 5 or 2, usually green, when 5 slightly unequal, when 2 strongly unequal, the ventral one much larger than the dorsal. Corolla white, pinkish, lilac, or creamy, sometimes pink- or red-striped or -spotted, sometimes yellow in the throat, infundibuliform (Guianan spp.), horizontal in the calyx, 3-6 $\times$ as long as the calyx, spurred or gibbous at the base, contracted above the spur or the gibbosity, contracted in the throat or not (Guianan spp.), ventricose or not, glabrous outside or nearly so; limb spreading; lobes 5 , subequal, rounded. Stamens 4 , didynamous, included; filaments glabrous, contorted or not, all connate at the base (Guianan spp.) or free from each other, ventrally adnate for several $\mathrm{mm}$; anthers about twice as wide as long, all coherent, coherent in pairs, or free; cells subglobose, $1-1.5 \times$ as long as wide, divaricate, separated by the enlarged connective, partially dehiscent in the middle or at the base. Ovary superior; style included; stigma bilobed or stomatomorphic. Disk gland one, dorsal, large. Placentae ovuliferous only on the inner or on both surfaces. Fruit a berry, globose or nearly so. Seeds often reddish, slightly shining, surrounded by a white diaphanous arillus, fusiform or ellipsoid, longitudinally or obliquely striate.

Type species: C. gracilis (Mart.) Hanst.

Distribution: About 15 species in tropical America.

Key to the species:

Calyx 5-parted; leafblades entire ..... 2. C. crassifolia Calyx 2-parted; leafblades near the apex mostly serrulate .

1. C. calcarata (Miq.) Hanst., Linnaea 34: 416. 1865 (type only); Pulle, Enum. 420. 1906; Benoist, Bull. Soc. Bot. France Sér. 4. 21 : 320. 1921; Sandwith, Kew Bull. 1931 : 490; Morton, Bull. Torr. Bot: 
Cl. 75: 564. 1948; Fieldiana 28: 1092. 1957; Lemée, Fl. Guian. fr. 3: 461. 1953.

Fig. 13 Ca, p. 379

Basionym: Nematanthus calcaratus Miq., Linnaea 22: 472. 1849. Type: Suriname: near plant. Berlijn, Suriname distr., Focke941 (U). Heterotypic synonym: Codonanthe bipartita L. B. Smith, Bull. Torr. Bot. Gl. 60: 657, f. 1-6. 1933. Type: BrITISH Guiana: Kartabo Region, Bailey 181 (GH, holotype, isotype: NY).
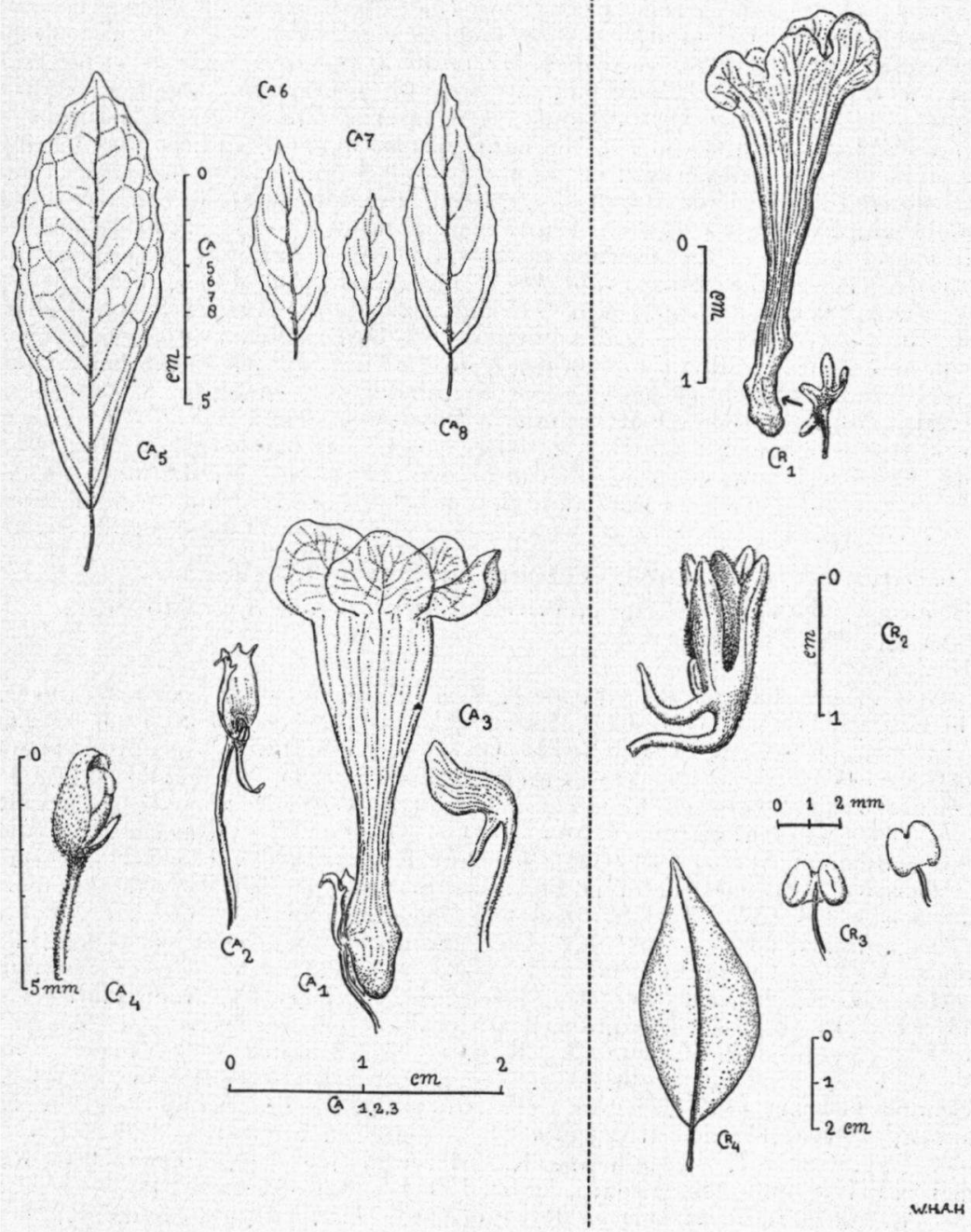

Fig. 13. Ca. Codonanthe calcarata: 1. flower (de la Cruz 1316); 2. calyx (de la Cruz 3735); 3. calyx (de la Cruz 1316); 4. flower-bud (A. C. Smith 2815); 5-8. leaves (5: Maguire \& Fanshawe 22838, 6: A. C. Smith 2815, 7-8; de la Cruz 1135). Cr. C. crassifolia (Mennega 560): 1. calyx and corolla; 2. immature fruit; 3. anther, both sides; 4 . leaf. 
Leafblades mostly serrulate near the apex, minutely puberulous to glabrous on both sides. Sepals 2, free, the dorsal one linear-lanceolate, $4-7 \times$ as lóng as wide, $2-4 \times 0.3-1 \mathrm{~mm}$, acute, entire, curved around the spur, the ventral one much larger, oblong or rectangular, $1.5-3 \times$ as long as wide, 3-7 $\times 1.5-4 \mathrm{~mm}$, rounded and entire or quadridentate at the apex. Corolla $3.5-6 \times$ as long as the calyx, 20-37 mm long.

Stems $0.15-1.00 \mathrm{~m}$ long, usually more woody than in the following species, puberulous at the apex, glabrescent. Leaves: petiole puberulous or glabrous, 1-9 $\mathrm{mm}$ long; blade dark green above, somewhat paler green or purple beneath, oblong-elliptic, 2.5-3.5 (mostly 3) $\times$ as long as wide, 15-115 $\times$ 5-40 mm, fleshy, membranaceous or papyraceous when dry, acute at the apex, cuneate at the base; veins inconspicuous; midrib prominent beneath. Flowers 1-8 together. Pedicels minutely puberulous, 7-17 mm long. Sepals green, puberulous or glabrous on both sides. Corolla white, pinkish, or light purple, sometimes pink- or red-striped and pink- or pale crimson-spotted, at the base 1-2 mm, at the throat 5-11 mm wide, spurred at the base, slightly contracted above the spur, not in the throat, strongly ampliate upwards, not ventricose, glabrous outside, inside sometimes with several hairs near the insertion of the stamens and minutely puberulous with glandular hairs in the throat; tube 16-25 mm long; spur obtuse, about $2 \times$ as long as wide, 2-4 $\times 1-2 \mathrm{~mm}$; limb 9-16 $\mathrm{mm}$ wide; lobes entire, 3-8 mm in diam. Filaments glabrous, contorted, all connate at the base, inserted on the base of the corolla and ventrally adnate to $5-6 \mathrm{~mm}$ from the base; anthers dumb-bell-shaped, $0.8 \times 1.5 \mathrm{~mm}$, coherent in pairs or free. Staminode very small, $0.2-0.3 \mathrm{~mm}$ long, glabrous, without anther. Ovary minutely puberulous, ovoid, $1.5-2 \times 1-1.5 \mathrm{~mm}$; style sparsely pubescent, 16-21 mm long; stigma stomatomorphic. Disk gland entire, $1 \times 0.5-1 \mathrm{~mm}$, glabrous. Placentae ovuliferous only on the inner surface. Berry purple, blue-black, or dark red, globose, glabrous, 8-12 $\mathrm{mm}$ in diam. Seeds slightly curved, $4 \times$ as long as wide, $3-4 \times 0.8-1 \mathrm{~mm}$, longitudinally striate.

Distribution: Venezuela, Guiana, northern Brazil.

Ecology: Epiphytic on arboreal ants' nests in rainforests; alt. 0-500 m.

British Guiana: Barima R., Matthews Ridge, Cowan 39358 (NY); Waini R., de la Cruz 3735 (NY, GH, US); Itabu Ck., East branch, Beddington $44(\mathrm{~K})$; Mt. Everard, de la Cruz 1316 (NY, PH, US); Pomeroon distr., Waramuri Mission, de la Cruz 1135 (NY); Upper Mazaruni R., de la Cruz 2294 (NY, F, GH, MO, US); Mazaruni Region, between Membaru Ck. and Makreba Falls on Kurupung R., Pinkus 244 (NY); Mazaruni Station, F.D. 4158 (K, NY); Mazaruni R., near Forest Settlement, Weber 347 (GH); Kartabo Region, Bailey 110 (GH, paratype of $C$. bipartita) 181 (GH, holotype of $C$. bipartita, isotype: NY); Penal Settlement, Hitchcock 17234 (NY, K, US); Kaieteur Savanna, Jenman 1067 (K); Potaro R., Tumatumari, Gleason 361 (NY, US); Basin of Essequibo R., near mouth of Oronoro Ck., A. C. Smith 2708 (NY, US), 2815 (NY, US); Upper Essequibo R., Mataruki R., Meyers 5817 (K); Essequibo R., Tiger Ck., Labbakabra Ck., Sandwith 1216 (K, U); Essequibo R., Moraballi Ck., near Bartica, Sandwith $286(\mathrm{~K})$; Essequibo R., Groete Ck., Kamuni Ck., Maguire \& Fanshawe 22838 (NY, A, F, K, U, US); southeast of Georgetown, Hitchcock 16948 (NY, US); Rockstone, Gleason 745 (NY), 854 (NY); Corantijn R., Epeiro, Jenman 236 (K). SuRiname: Maratakka R., B.W. 3486 (U); island in Lucie R., Hulk 416 (U); Upper Coppename R., Tanjimama R., Mennega 378 (U); Upper Gran Rio, Stahel 248 (U); ibid., Maupé-dam, Stahel 180 (U); Saramacca R., Pulle 134 (U); above Awana-Falls in Saramacca R., Florschütz 1181 (U); Suriname R., near Kadjoe, coll. indig. 255 (U); Patrick-savanna near Brownsberg, Lanjouw 1244 (U); Zanderij I, Florschütz 613 (U); ibid., Maguire \& Stahel 25050 (NY, U, US); Para distr., Went 379 (U); Para R., Onoribo, Suringar, 1 Jan. 1885 (L); Berlijn, Focke 941 (U, holotype).

French Guiana: Lower Mana R., Charvein, Benoist 57 (P); Cottica Mts., Versteeg 
329 (U); de Candolle \& l'Héritier s.n. (G); Martin s.n. (BM); Poiteau s.n. (K); L. C. Richard s.n. (P).

Brazil: Amapá: Rio Amapari, Serro do Navio, Cowan \& Maguire 38055 (NY); Cowan 38327 (US), 38555 (US).

2. C. crassifolia (Focke) Morton, Field Mus. Publ. Bot. 18: 1159. 1938; Bull. Torr. Bot. Cl. 75: 564. 1948; Fieldiana 28: 1092. 1957; Lemée, Fl. Guian. fr. 3: 461. 1953. Fig. $13 \mathrm{Cr}$, p. 379

Basionym: Hypocyrta crassifolia Focke, Tijdschr. Nat. Wetensch. 5: 199. 1852.

Type: Suriname: Focke, coloured drawing (L, photographs: U, WAG).

Heterotypic synonyms: Codonanthe confusa Sandwith, Kew Bull. 1931 : 492. Type: French Guiana: Lower Mana R., Acarouany R., Sagot 426 (K, holotype, isotypes: BM, BR, P, 4 sheets, U, W, 2 sheets, photographs of one $P$ sheet in U and US).

Misapplied name: Codonanthe calcarata Hanst., Linnaea 34: 416. 1865, exclusive of type.

Episcia hookeri Hanst., Linnaea 34: 350. 1865; Morton in Fl. Trin. and Tob. 2 (5): 309. 1955. Type: Hooker, Icones 9: 873. 1852, aberrant!

Misapplied name: Centrosolenia glabra Hook., Icon. cit., not Benth.

Leafblades entire or rarely obscurely sinuate, glabrous on both sides. Sepals 5, free, linear-lanceolate, $4-8 \times$ as long as wide, 2.5-9 $\times$ $0.5-1.5 \mathrm{~mm}$, slightly unequal, acute at the apex, entire, four erect, oblique, the fifth (dorsal) curved around the spur. Corolla $3-4 \times$ as long as the calyx, 15-28 $\mathrm{mm}$ long.

Stems 0.15-2.00 m long, puberulous at the apex, glabrous. Leaves: petiole puberulous or glabrous, $2-15 \mathrm{~mm}$ long; blade green, sometimes reddish, oblongovate or oblong-elliptic, $1 \frac{1}{2}-3$ (mostly $2 \frac{1}{2}$ ) $\times$ as long as wide, $20-85 \times 6-39 \mathrm{~mm}$, fleshy, chartaceous when dry, acute or obtuse at the apex, cuneate or rounded at the base; veins inconspicuous; midrib prominent beneath. Flowers 1-4 together. Pedicels puberulous, 5-11 mm long. Sepals green, puberulous on both sides. Corolla white or yellowish, sometimes pink-striped outside, yellow in the throat (testibus Focke et Lindeman), at the base 1-1.5 mm, at the throat 3-6 mm wide, spurred at the base, contracted above the spur, not so in the throat, strongly ampliate upwards, not ventricose, puberulous or glabrous outside, inside with an irregular ring of glandular hairs in the throat; tube 12-18 $\mathrm{mm}$ long; spur obtuse, about $2 \times$ as long as wide, 2-4 $\times 1-2 \mathrm{~mm}$; limb oblique, 6-15 $\mathrm{mm}$ wide; lobes entire, 2-6 mm in diam. Filaments glabrous, contorted, gradually widened towards the base, all connate at the base, inserted on the base of the corolla and ventrally adnate to 3-4 mm from the base; anthers creamy, nearly reniform, $0.8 \times 1.5 \mathrm{~mm}$, coherent in pairs or free. Staminode very small, glabrous, $0.2-0.7 \mathrm{~mm}$ long, without or with a sterile anther. Ovary puberulous, oblong-ovoid, 2-4 $\times 1-2 \mathrm{~mm}$; style shorter than the stamens, glabrous, 8-10 mm long, deciduous; stigma stomatomorphic. Disk gland entire, $1 \times 0.5-1 \mathrm{~mm}$, glabrous. Placentae ovuliferous only on the inner surface. Berry red, subglobose, practically glabrous, 10-12 $\times 7-9 \mathrm{~mm}$. Seeds reddish, slightly curved, $3 \times$ as long as wide, $2 \times 0.7 \mathrm{~mm}$, longitudinally striate.

Distribution: Mexico, Central America, Colombia, Venezuela, Guiana, and Trinidad.

Ecology: Epiphytic on ants' nests on tree trunks in rain- or swampforests; alt. $0-1400 \mathrm{~m}$. 
Venezuela: Delta Amacuro: Rio Manimo, Bond, Gillin \& Brown 219 (PH, NY). British Guiana: Kabakaburi, Pomeroon distr., de la Cruz 3250 (NY, GH, PH, US); Upper Mazaruni R., de la Cruz 2197 (NY, F, US); ibid., Kamakusa, de la Cruz 2810 (NY, GH, PH, US); Potaro R., Post above Kaieteur Falls, Sandwith 1385 (K); Upper Rupununi R., near Dadanawa, de la Cruz 1405 (NY, US); Basin of Kuyuwini R., Essequibo trib., A. C. Smith 3026 (NY, F, G, K, MO, S, U, US); Essequibo R., Moraballi Ck., near Bartica, Sandwith 316 (K); Macouria R., Lower Essequibo R., Jenman 2517 (K); Jenman 7983 (K).

Suriname: Kabalebo R., Avanavero Falls, Pulle 444 (U); Maratakka R., B.W. 3489 (U); Upper Nickerie R., B.W, 1063 (U); Lucie R., Hulk (?) s.n: (U, spirit coll.); Coronie, Lanjouw \& Lindeman 1410 (U); Tibiti savanna, Lanjouw \& Lindeman 1940 (U); between Pakka Pakka and Ebbatop, near fall in Kleine Saramacca R., Florschütz 1522 (U); Tapanahoni R., near Granholo Falls, Florschütz 515 (U); Litanie R., near Knopaia Moi, Versteeg 394 (U); Litanie R., near Jamaiké, Rombouts 790a (U); Nassau Mts., bank of Marowijne R., Lanjouw \& Lindeman 2104 (U) ; Jodensavanne-Mapane Ck. area, Suriname R., Lindeman 3613 (U), 5186 (U); ibid., Suhoza, Mennega 564 (U); Republiek, Kuyper 1 (U); N.W. of Lelydorp, $25 \mathrm{~km}$ south of Paramaribo, Jonker 308 (U), 314 (U); Paramaribo, Boldingh 3825 (U); ibid., Weigelt, anno 1827 (PH, W); ibid., Maguire \& Stahel 22765 (A, F, K, U, US); ibid., Beekhuizen, Wullschlägel 379 (BR, GOET, U, W); ibid., near plant. Geyersvlijt, Kegel 805 (GOET); ibid., plant. Peperpot, Mennega 560 (U, spirit coll.); herb. Endlicher s.n. (W); Focke, drawing ( $\mathrm{L}$, type); Hostmann $285(\mathrm{~K})$.

French Gutana: Lower Mana R., Acarouany R., Sagot 426 (K, holotype of C. confusa; isotypes: BM, BR, P, U, W); ibid., Charvein, Benoist $194(\mathbf{P})$.

\section{Columnea L., Sp. Pl. 638. 1753.}

Epiphytic or terrestrial shrubs, subshrubs, vines, or herbs of very variable size, up to $5 \mathrm{~m}$ long when climbing, when erect up to $1.50 \mathrm{~m}$ high. Stems erect, ascending, creeping, or pendant, branched or not, mostly with spreading adventitious roots. Leaves opposite, those of a pair equal to strongly unequal, petiolate or subsessile, very variously shaped, ovate or oblong-elliptic to linear-lanceolate. Flowers axillary, solitary or fasiculate, short- or long-pedicellate, with or without bracts. Sepals 5 , usually coloured, free or connate at the base, acute or acuminate, often toothed or incised. Corolla variously coloured, erect in the calyx, often gibbous-but not spurred-at the base; tube ampliate upwards or not, usually not ventricose; limb actinomorphic, subactinomorphic, or slightly to strongly bilabiate. Stamens 4, didynamous, included or exserted; filaments all connate at the base, usually not adnate to the corolla except at the very base; anthers mostly all coherent, in bilabiate corollas covered by the dorsal lip, sometimes free, quadrate, oblong or nearly so; cells parallel, discrete, dehiscent throughout by a longitudinal split. Ovary superior; style mostly about as long as the stamens, included or exserted; stigma mostly bilobed, in bilabiate corollas above the anthers and covered by the dorsal lip. Disk glands 1-5, mostly one large dorsal one. Placentae ovuliferous only on the inner or on both surfaces. Fruit a berry, globose or nearly so. Seeds shining, ellipsoid or fusiform, longitudinally or obliquely striate.

Type species: $C$. scandens $\mathrm{L}$.

Distribution: About 160 species in tropical America. 
Key to the species:

1. Leaves of a pair strongly unequal . . . . . . . . . . 2

Leaves of a pair equal or subequal . . . . . . . . . . 3

2. Blade of larger leaf sparsely pilose above, partially purple beneath ............ 3. C. guianensis Blade of larger leaf hirsute above, plain green beneath . . 1. C. aureonitens

3. Corolla red; limb strongly bilabiate; plant not conspicuously hairy; leaves small, usually shorter than the flowers ...

Corolla yellow; limb subactinomorphic; plant hirsute; leaves longer than the flowers ...... 2. C. calotricha

4. Pedicels mostly about as long as the leaves; sepals ovate, $1.5-3$ $X$ as long as wide; leafblades acute or acuminate at the apex, revolute at the margin, glabrous above : . . . . . . . . . . . . . . . . 4. C. oerstediana Pedicels much shorter than the leaves; sepals lanceolate or linear-lanceolate, $2.5-10$ (mostly about 5) $\times$ as long as wide; leafblades acute or obtuse at the apex, usually not revolute, strigose on both sides . . . . 5. C. scandens

1. C. aureonitens Hook., Bot. Mag. 73: t. 4294. 1847; Hanstein, Linnaea 34: 386. 1865.

Type: Hooker, Bot. Mag., Icon. cit., no specimen preserved; also none of the specimens mentioned by HoOKER is at present at $K$. Homotypic synonyms: Collandra aureonitens (Hook.) Hanst., Linnaea 26: 209. 1853.

Columnea pilosa Lem., Fl. d. Serres Sér. 1. 3: t. 223.1847.

Collandra pilosa Lem., l.c. (published in synonomy).

Leaves of a pair strongly unequal, the larger one about $10 \times$ as long as the other, shortly petiolate; blade dark green above, paler green beneath, oblique, lanceolate, densely strigose above, subtomentose beneath, especially on the midrib and veins; the smaller one stipule-like, subsessile, ovate-lanceolate. Flowers solitary or aggregated. Bracts lanceolate, laciniate, about as long as the sepals and similarly shaped. Sepals green, often reddish by the hairs, lanceolate. Corolla yellow, inside seemingly with one red spot on each lobe due to the hairs on the outside, softly hirsute outside with reddish-brown or golden-shining hairs, nearly cylindric; limb erect, actinomorphic. Stamens included.

Terrestrial or epiphytic subshrub. Stems sometimes with spreading adventitious roots, tomentose or softly hirsute at the apex, glabrescent. Leaves: larger one:

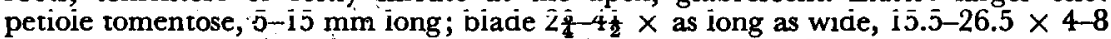
$\mathrm{cm}$, papyraceous when dry, acuminate at the apex, obliquely cuneate at the base, serrate-dentate; smaller one: similarly coloured, $2 \frac{1}{2}-4 \times$ as long as wide, $20-38 \times$ 6-14 mm, long-acuminate, obliquely rounded at the base, subentire or obscurely serrate. Sepals free or nearly so, subequal, $3-5 \times$ as long as wide, $12-20 \times 3-4 \mathrm{~mm}$, acuminate at the apex, slightly narrowed near the base, laciniate, softly reddishbrown-hirsute on both sides, especially outside. Corolla slightly oblique in the calyx, 11-2 $x$ as long as the calyx, 24-30 (according to Hooker -38) $\mathrm{mm}$ long, at the base and at the throat 5-6 mm wide, gibbous at the base, slightly contracted above the gibbosity and in the throat, hardly ventricose, somewhat bent down- 
wards, inside partially pubescent with glandular hairs; tube $21-27 \mathrm{~mm}$ long; lobes equal, oval, entire, $2.5-4 \times 2-2.5 \mathrm{~mm}$. Filaments pilose or glabrous, contorted or not, all connate at the base for $2.5-6 \mathrm{~mm}$, inserted on the base of the corolla; anthers all coherent, orbicular or broader than long. Staminode very small, 0.5-1 mm long, glabrous, with a triangular sterile anther. Ovary hirsute, ovoid, 4-5 $\times 2.5-3 \mathrm{~mm}$; style included, glabrous or nearly so; stigma bilobed. Disk glands 1-5; two dorsal ones mostly united, three others (figured and described by HOOKER) smaller, but longer, approximately tongue-shaped, wanting in the examined specimens. Placentae ovuliferous on the inner surface only. Berry white (teste Steyermark), sugblobose, sparsely pubescent, when dry, $15 \times 10 \mathrm{~mm}$. Seeds dark brown, ellipsoid, $2 \frac{1}{2}-3 \frac{1}{2} \times$ as long as wide, $1.2-1.4 \times 0.4-0.55 \mathrm{~mm}$, obliquely striate.

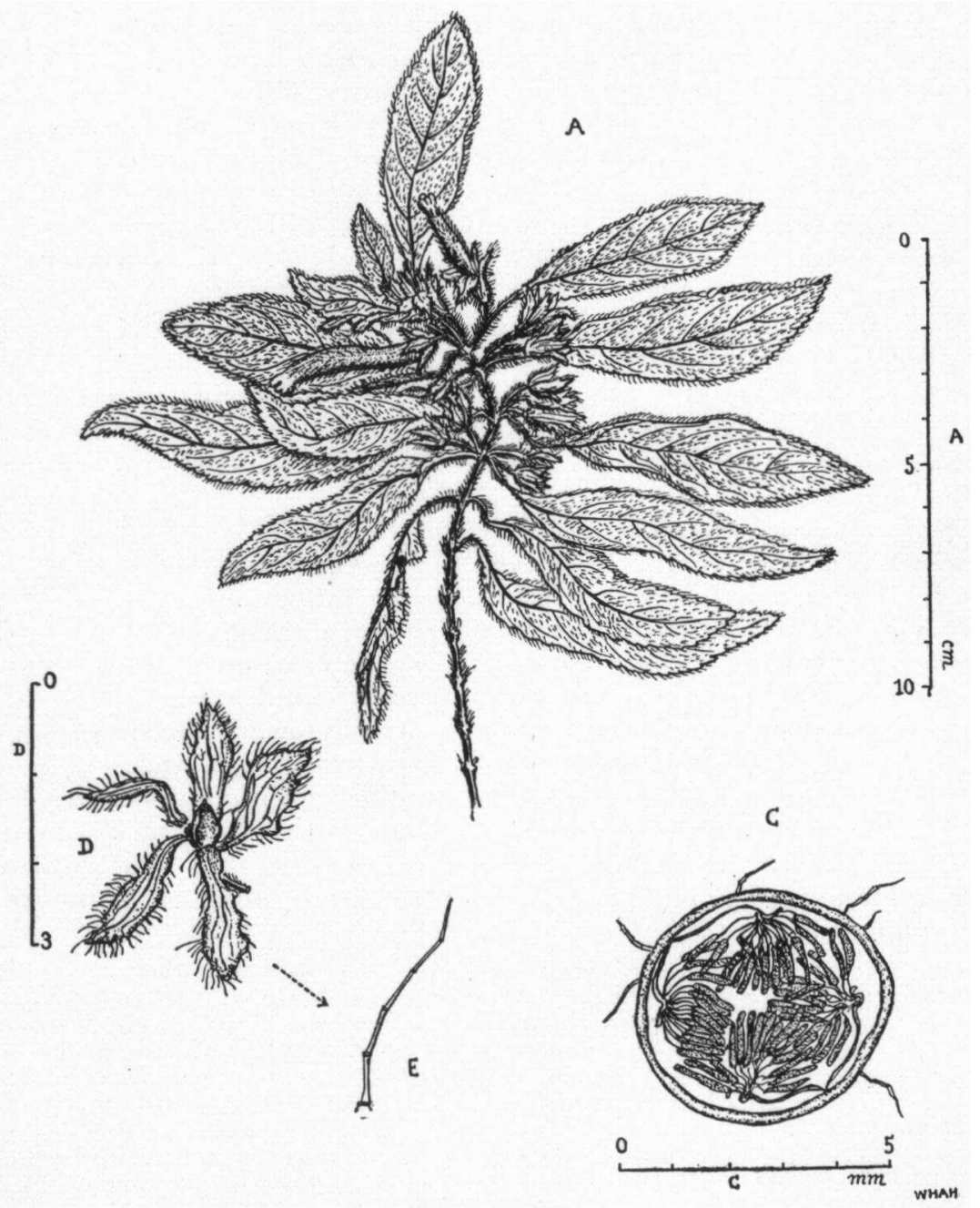

Fig. 14. Columnea calotricha: A: branch (B. W. 5723); C. transverse section of immature fruit; D. immature fruit; E. hair (C-E. Lanjouw \& Lindeman 2742). 
Distribution: northern Colombia and Venezuela. ${ }^{1}$ )

Ecology: in montane rainforests; alt. about $1000-1500 \mathrm{~m}$.

Description based on Colombian and Venezuelan material. From Guiana only known by a vegetative specimen:

SuRINAME: Wilhelmina Mts., B.W. 7059 (U).

This might be $C$. affinis Morton described from Venezuela which differs chiefly by its red bracts and calyx, or another species.

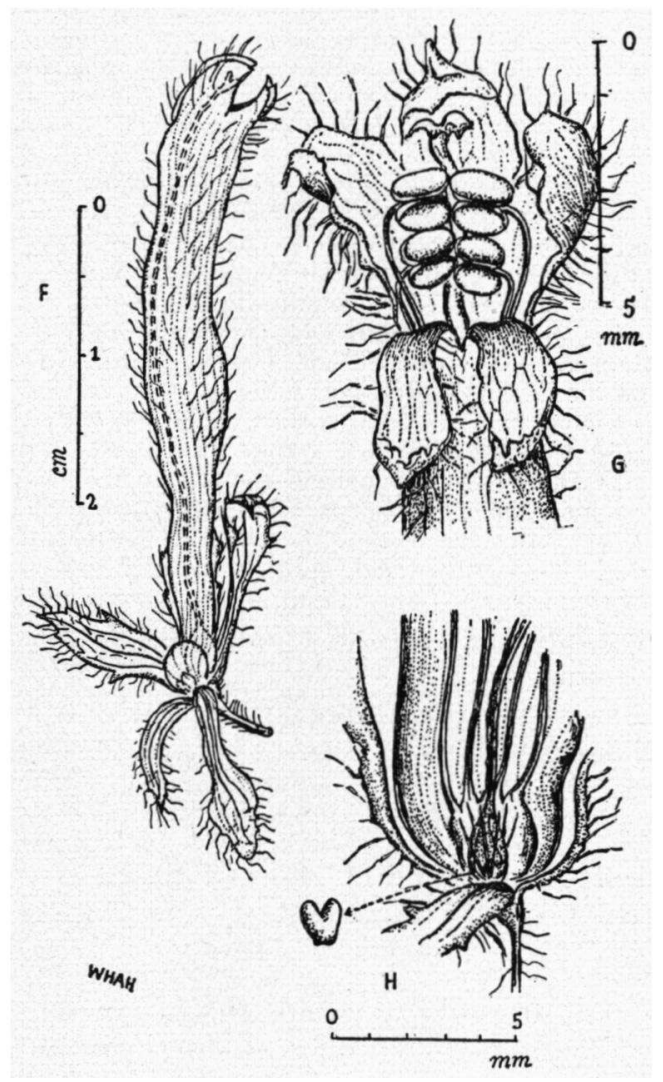

Fig. 15. Columnea calotricha: F. flower; G. upper portion of flower; H. lower portion of flower, opened (F and H: B. W. 5723, G: Lanjouw \& Lindeman 2921).

2. C. calotricha Donn. Sm., Bot. Gaz. 40: 9. 1905; Morton, Field Mus. Publ. Bot. 18: 1163. $1938 . \quad$ Figs. 14, p. 384; 15, p. 385 Type: Guatemala: Dept. Alta Verapaz, Cubilquitz, von Tuerckheim 8542 (US, 2 sheets).

1) The Central American specimens identified as $C$. aureonitens belong to an undescribed species that is closely related. This differs from it by the following characters: Corolla about $3-4 \times$ as long as the calyx, pilose outside; stamens exserted, anthers nearly rectangular, longer than wide. 
Plant hirsute. Leaves of a pair subequal, petiolate; blade dark green above, paler green, purplish, or red beneath, oblong-elliptic or lanceolate. Flowers 1-7 together. Sepals reddish, often green at the base, spathulate-oblong. Corolla yellow with brown hairs outside, narrowly tubular; limb obscurely bilabiate; lobes five, subequal, erect, two dorsal ones concave. Stamens exserted when filaments not contorted.

Epiphytic or terrestrial shrub, subshrub, or herb, 0.20-1.00 m high. Stems erect or ascending, branched, with spreading adventitious roots, hirsute. Leaves: petiole hirsute, 5-25 mm long; blade 2.5-6 (mostly 4) $\times$ as long as wide, 3-16 $\times 1-4 \mathrm{~cm}$, papyraceous when dry, acute at the apex, obliquely cuneate at the base, remotely crenulate, hirsute on both sides. Inflorescence: pedicels hirsute, 3-10 mm long. Sepals free, subequal, $4-4.5 \times$ as long as wide, $12-25 \times 3-6 \mathrm{~mm}$, acute at the apex, narrowed towards the base, serrate near the apex, hirsute outside, inside only above. Corolla erect in the calyx, $1.5-3 \times$ as long as the calyx, $2-4 \mathrm{~cm}$ long, extending its length during anthesis!, at the base $2-3 \mathrm{~mm}$, at the throat $2-5 \mathrm{~mm}$ wide, gibbous at the base, slightly contracted above the gibbosity and in the throat, not or hardly curved, not ventricose, hirsute outside except for the glabrous basal part, glabrous inside; tube 17-35 mm long; lobes oblong, 3-4 $\times 2-3 \mathrm{~mm}$, acute, entire. Filaments glabrous, contorted or not, equalling the corolla tube, all connate at the base, inserted on the base of the corolla; anthers orbicular or nearly so, all coherent, covered by' the dorsal corolla lobes when filaments not contorted. Staminode none. Ovary sparsely, hirsute, ovoid, $3 \times 2 \mathrm{~mm}$; style filiform, glabrous, about as long as the stamens; stigma bilobed, covered by the dorsal corolla lobes. Disk gland one, dorsal, large, entire or bilobed, glabrous. Placentae ovuliferous on the inner surface only. Berry red, ovoid, with scattered hairs, $10 \times 5 \mathrm{~mm}$ (16 mm long, teste J. D. Smith). Seeds (immature ?) dark brown, fusiform, about $6 \times$ as long as wide, $1.2 \times 0.2 \mathrm{~mm}$, longitudinally or slightly obliquely striate.

Distribution: Only known from Guatemala, Costa Rica, Suriname, and French Guiana.

Ecology: In rain- and swampforests, at low and middle elevations. SURINAME: Brownsberg, B.W. 626 (U), 664 (U), 6619 (U) ; Nassau Mts., Lanjouw \& Lindeman 2742 (U), 2921 (U); Emmaketen, B.W. 5723 (U).

French Gulana: Martin 275 (BM), s.n. (BM); Martin, herb. Rudge s.n. (BM).

3. C. guianensis Morton, Bull. Torr. Bot. Cl. 75: 564. 1948.

Type: British Gutana: Potaro :R. Gorge, Maguire \& Fanshawe 23067 (F, K, MO, NY, 2 sheets, U; isotypes). $\quad$ Fig. 16, p. 387

Leaves of a pair strongly unequal, the larger one about 10-15 $\times$ as long as the other, shortly petiolate; blade medium (?) green above, beneath usually partially red above the middle, oblanceolate, minutely pilose above when young, soon glabrous, beneath pilose with thin hairs; the smaller one stipule-like, subsessile, ovate-lanceolate. Flowers 1-3 together. Bracts of approximately the same size and shape as the smaller leaves. Sepals green, lanceolate. Corolla pale yellow, with outside crimson lobes, narrowly cylindric; limb erect, subactinomorphic. Stamens included.

Epiphytic or terrestrial (?) procumbent and ascending perennial herb. Stems with spreading adventitious roots, villose or hirsute, eventually glabrescent. Leaves: petiole of the larger one villose or hirsute, 4-10 mm long; its blade 3-4 $\times$ as long as wide, $15-27 \times 5-8.5 \mathrm{~cm}$, chartaceous when dry, minutely denticulate, shortly acuminate at the apex, very obliquely cuneate at the base; the smaller one about $3 \times$ as long as wide, $15-30 \times 5-10 \mathrm{~mm}$, argutely acuminate at the apex, very obliquely rounded or subcordate at the base, nearly entire, practically glabrous 
above, pilose beneath. Inflorescence: pedicels villose, 5-10 mm long. Sepals leafy, hardly connate at the base, subequal, erect, 4-5 $\times$ as long as wide, $20-25 \mathrm{~mm}$ long, long-acuminate, sharply serrate, somewhat narrowed towards the base, yellowvillose on both sides, the dorsal one $4 \mathrm{~mm}$, the others 5-6 mm wide. Corolla erect in the calyx, 25-33 mm long, dorsally obscurely gibbous at the base, slightly contracted above the gibbosity and in the throat and there 4-6 mm wide, yellowsericeous outside, sparsely puberulous inside; lobes five, entire, about $2 \mathrm{~mm}$ long and wide, three free, nearly triangular, rounded at the apex, two dorsal ones almost to the apex connate. Filaments contorted, at the base for $2.5-4 \mathrm{~mm}$ connate, inserted on the base of the corolla, with scattered minute hairs; anthers subquadrate, free; connective minutely pilose. Staminode very small, $0.2 \mathrm{~mm}$ long, tongue-shaped, acuminate, glabrous, without anther. Ovary pubescent, ovoid, $4 \times 2.5-3 \mathrm{~mm}$; style included, glabrous; stigma bilobed. Disk gland one, dorsal,

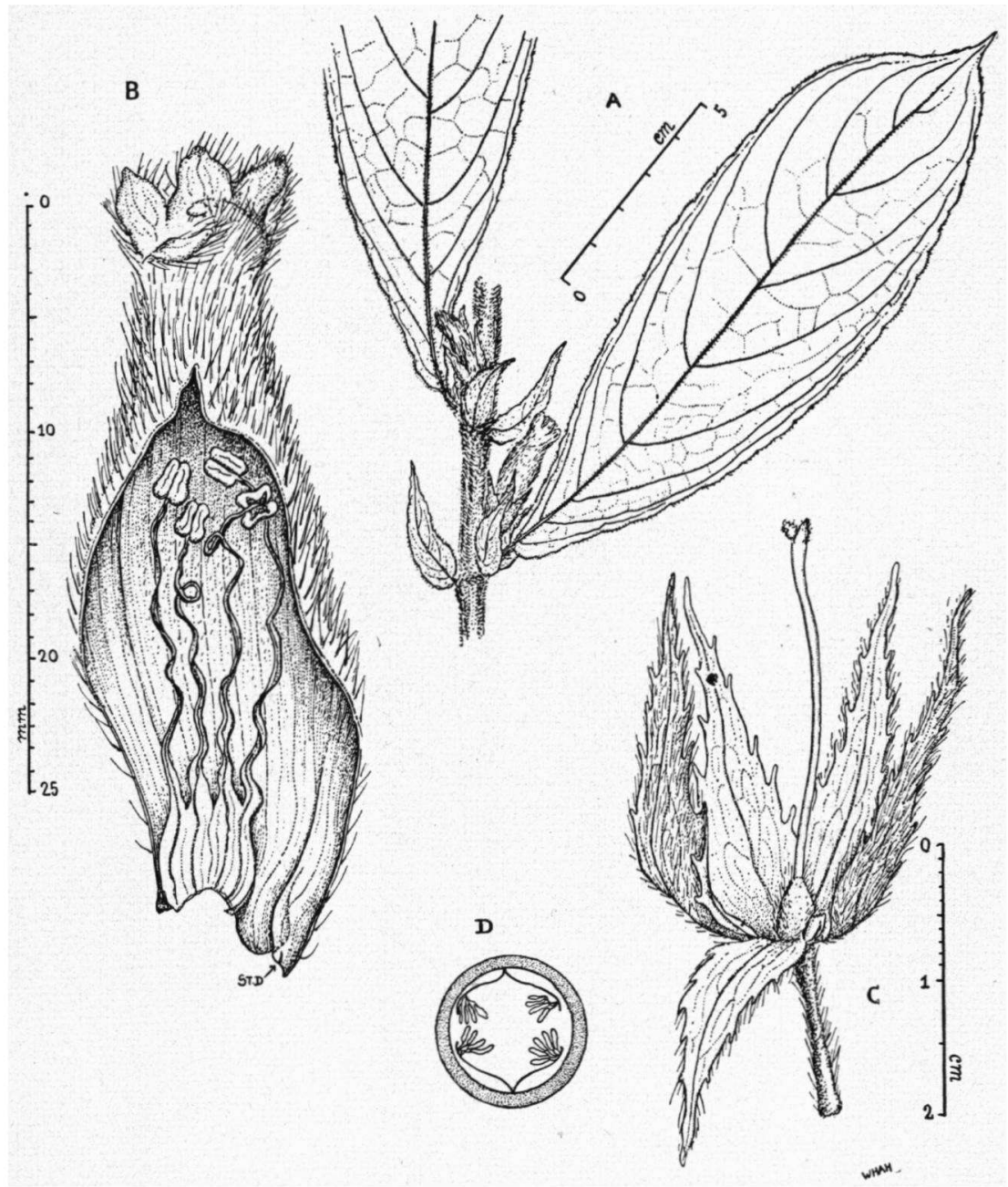

Fig. 16. Columnea guianensis (Maguire \& Fanshawe 23067): A. branch; B. opened corolla; C. calyx with pistil; D. diagrammatic transverse section of ovary. ‘ 
large, emarginate, glabrous, about $1.5 \mathrm{~mm}$ high. Placentae ovuliferous on the inner surface only. Fruit a berry (?), (immature ?) ellipsoid, pubescent, $10 \times 6 \mathrm{~mm}$. Seeds (immature ?) dark brown, ellipsoid, about $4 \times$ as long as wide, $1.2 \times 0.3$ $\mathrm{mm}$, longitudinally striate.

Ecology: In montane rainforests; at higher elevations.

Only known from the following collections:

British Guiana: Potaro R. Gorge, Maguire \& Fanshawe 23067 (F, K, MO, NY, 2 sheets, $U$; isotypes).

Brazil: Rio Branco: Roraima, Ule 8760 (K).

4. C. oerstediana Klotzsch ex Oerst., Centralamer. Gesn. 61, t. 8. 1858; Hanstein, Linnaea 34: 407. 1865; Prain, Bot. Mag. 136: t. 8344. 1910; Morton, Field Mus. Publ. Bot. 18: 1167. 1938.

Fig. 17, p. 388

Type: Costa Rica: Naranjo, Oersted 9291 (G, holotype, photographs: U, US, isotypes: C, 2 sheets, US, 2 sheets, photograph of one $\mathrm{G}$ sheet in US).

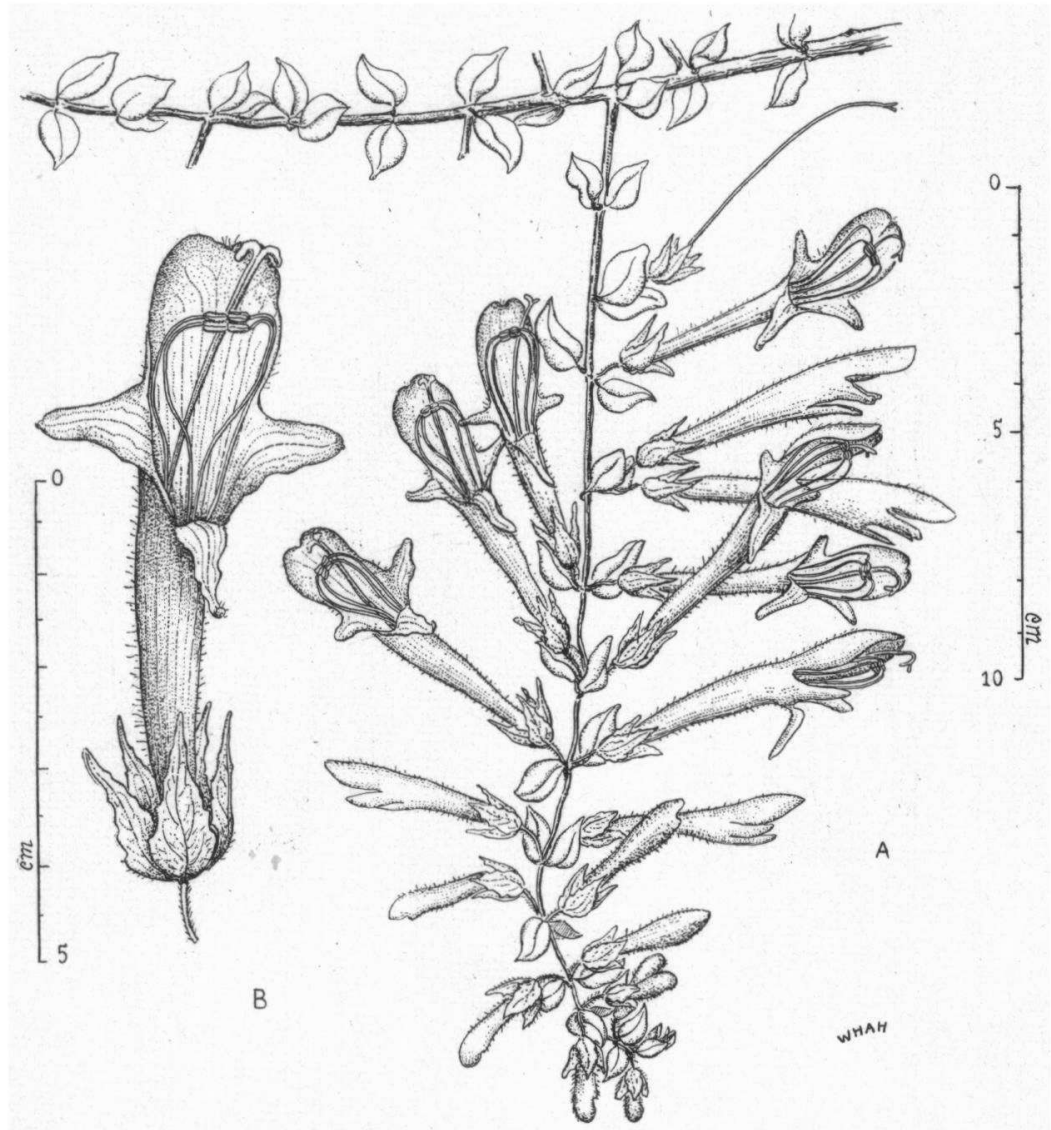

Fig. 17. Columnea oerstediana (Skutch 3554): A. branch; B. flower. Usually fewer flowers and longer pedicels. 
Leaves of a pair equal, small, shortly petiolate; blade ovate, oblong-ovate or oblong-elliptic, $10-37 \mathrm{~mm}$ long, shining and glabrous above, minutely appressed-pubescent beneath, somewhat revolute at the margin. Flowers solitary. Pedicels about as long as the leaves, 6-37 (mostly 10-25) mm long. Sepals green or somewhat reddish, ovate, $1.5-3 \times$ as long as wide, rounded or subcordate at the base. Corolla orange or orange-red, about $5 \times$ as long as the calyx; limb strongly bilabiate. Stamens exserted.

Epiphytic subshrub or herb. Stems pendant; creeping, or erect, $0.30-0.50 \mathrm{~m}$ long or more(?), branched, sometimes with nodigenous adventitious roots, appressedpubescent at the apex, glabrescent. Leaves: petiole appressed-pubescent, $0.5-2 \mathrm{~mm}$ long; blade deep green above; pale green beneath, 1.5-3 $\times$ as long as wide, fleshy, chartaceous or coriaceous when dry, acute or acuminate at the apex, cuneate or rounded at the base, entire; primary veins 1-4 on each side, curved along the margin. Inflorescence: pedicels appressed-pubescent. Sepals free, 10-18 $\times 3-11 \mathrm{~mm}$, acuminate at the apex, dentate, especially towards the base, or sinuate, sparsely pubescent on both sides. Corolla 45-70 mm long, at the throat 7-9 mm wide, gibbous at the base, slightly contracted above the gibbosity and there $2-4 \mathrm{~mm}$ wide, not contracted in the throat, somewhat bent downwards, slightly widened towards the throat, pilose outside, glabrous inside; tube narrow, 20-40 mm long; gibbosity pale green, rounded, $3 \mathrm{~mm}$ in diam.; ventral lip reflexed, of one lobe which is lanceolate, about $4 \times$ as long as wide, 12-16 $\times 3-4 \mathrm{~mm}$, obtuse, entire; dorsal lip erect, 3-lobed, $20-30 \mathrm{~mm}$ long, two lateral lobes spreading, obliquely triangular, about as long as wide, 5-11 mm long, at the base 5-10 mm wide, obtuse, entire; central lobe, the galea, about $11 \times$ as long as wide, $10-20 \times 7-15 \mathrm{~mm}$, entire, rounded, slightly cuspidate or retuse at the apex. Stamens yellow; filaments glabrous, not contorted; all connate at the base, inserted on the base of the corolla; anthers oblong, $1 \frac{1}{2}-2 \times$ as long as wide, all coherent, covered by the galea. Staminode none. Ovary pubescent, ovoid, 3-4 $\times 2-2.5 \mathrm{~mm}$; style exserted, as long as the corolla, minutely pubescent, apically curved along the inside of the galea; stigma bilobed. Disk gland one, dorsal, emarginate, glabrous. Placentae broad, ovuliferous on the inner surface only. Berry pubescent, $6-8 \mathrm{~mm}$ in diam. Seeds embedded in the fruit, covered by large hyalinous arilli (?), dark brown, ellipsoid, $1.2 \times 0.4 \mathrm{~mm}$, longitudinally striate.

Distribution: Only known from Costa Rica, French Guiana, and Amapá.

Ecology: In rainforests, at low elevations.

French Guiana: Sinnemary R., Aublet s.n. (BM), identified as C. scandens; near the confluence of Gêne R. and Oyac R., Martin s.n: (BM); Mt. Kaw, Cowan 38756 (U); Cayenne, Martin 100 (BM, p.p.), s.n. (BM, K); herb. Moricand s.n. (G); L. C. Richard s.n. (P). Guiana (FrENCH ?): Forster, herb. J. E. Smith 1102.4 (LINN).

Brazil: AMAPÁ: Rio Amapari, Serro do Navio, Cowan \& Maguire 38137 (NY); ibid., Cowan 38295 (US).

5. C. scandens L., Sp. Pl. 638. 1753; Jacquin, Hort. Vindob. 27, t. 48. 1776; Sims, Bot. Mag. 39: t. 1614. 1814; Loddiges, Bot. Cab. 5: t. 403. 1820; Edwards, Bot. Reg. 10: t. 805. 1824; Martius, Nov. Gen. 3: 65, 't. 226. f. 2. 1829; G. Don, Gen. Syst. 4: 652. 1838; D.C., Prod. 7: 542. 1839; Hanstein, Linnaea 26: 187, 211. 1853; Hooker, Bot. Mag. 85: t. 5188. 1859; Grisebach, Fl. Br. W.I. Isl. 464. 1861 ("1864"); Houllet, Rev. Hortic. 1864: pl. 231; Hanstein, Linnaea 34: 404. 1865; Duss, Fl. Ant. fr. 432: 1897; Urban, Symb. Ant. 2: 360. 1901; Morton, Fieldiana 28: 531. 1953; in Fl. Trin. and Tob. $2(5): 312.1955$. 
Neotype: Burmann, Plant. Amer. Car. Plumier. 1756. t. 139. f. 1, "Columnea".

Homotypic synonym: Glycanthes scandens (L.) Raf., Sylva Tellur. 83. 1838.

Heterotypic synonyms: Columnea rotundifolia Salisb., Parad. Lond. 1 (1): t. 29. 1806; Hanstein, Linnaea 34: 404. 1865; Urban, Symb. Ant. 2: 360. 1901. Type: TrINIDAD: Salisbury, icon. cit. (no specimen preserved).

C. jacquiniana Presl, Abh. Böhm. Ges. Wiss. 5 (3): 576. 1845. Type: Jacquin, Hort. Vindob. t. 48. 1776 (specimen after which it might have been depicted: Herb. Jacquin, from Martinique (BM).).

C. speciosa Presl, l.c., p. 575; Grisebach, Fl. Br. W.I. Isl. 464. 1861 ("1864"). Type not seen (mentioned as: "cult. in hortis").

C. fendleri Sprague, Kew Bull. 1912: 41; Morton, Fieldiana 28: 529. 1953. Type: Venezuela: Mérida ?, near Tovar, Fendler 2031 ( $\mathrm{K}$, holotype, isotype: GOET).

C. aripoensis Britton, Bull. Torr. Bot. Cl. 53: 470. 1926. Type: Trinidad: Heights of Aripo, Britton \& Freeman 2340 (NY).

Homotypic synonym: C. scandens var. aripoensis (Britton) Morton, Contrib. U.S. Nat. Herb. 29: 14. 1944; in Fl. Trin. and Tob. 2 (5): 313. 1955.

C. scandens var. vincentina Morton, Contrib. U.S. Nat. Herb. 29: 13. 1944. Type: St. Vincent: Mt. St. Andrews, Eggers 6698 (US, holotype, isotype: $\mathrm{P}$ ).

C. scandens var. australis Morton, Fieldiana 28: 531. 1953. Type: Venezuela: Monagas, Montaña de Aguacate, between Garipe and Caripito, Steyermark 62229 (F, holotype, isotype: US).

Leaves of a pair equal or sometimes subequal, petiolate; blade elliptic or oblong-elliptic, $15-65 \mathrm{~mm}$ long, strigose to glabrous or sometimes subtomentose on both sides. Flowers solitary, sometimes 2-3 together. Pedicels longer than the petioles, up to half as long as the leaves. Sepals green or sometimes reddish, ovate-lanceolate, lanceolate, or linear, 2.5-10 $\times$ as long as wide. Corolla red, 4-6 $\times$ as long as the calyx; limb strongly bilabiate. Stamens exserted.

Epiphytic or terrestrial, climbing or pendant vine or subshrub. Stems sarmentose, $30-50 \mathrm{~cm}$ long or more (?), branched, with spreading adventitious roots, sericeousstrigose near the apex, glabrescent. Leaves: petiole sericeous-strigose, $2-11 \mathrm{~mm}$ long; blade medium green above, mostly paler beneath, 1.4-3.5 $\times$ as long as wide, 9-38 mm wide, papyraceous or chartaceous when dry, acute or obtuse at the apex, cuneate or rounded at the base, entire, subentire, or remotely and shallowly crenate-dentate; primary veins 3-4 pairs, curved along the margin. Inflorescence: bracts, if any, small, green, lanceolate, $3-4 \times$ as long as wide, $4-8 \times 1.5-2 \mathrm{~mm}$, strigose, especially beneath. Pedicels sericeous-strigose, 8-20 mm long. Sepals free, at least the dorsal one, the others mostly connate for 1-2 mm, erect, subequal, acuminate, subulate, or sometimes acute at the apex, entire or with some teeth near the base, 8-17 $\times 1-4 \mathrm{~mm}$, strigose on both sides, especially outside. Corolla erect in the calyx, 43-70 mm long, extending its length during anthesis, dorsally gibbous at the base, slightly contracted above the gibbosity and there $1.5-4 \mathrm{~mm}$ wide, not contracted in the throat, at the throat 5-9 $\mathrm{mm}$ wide, pilosulous outside, inside only dorsally minutely and sparsely pubescent; tube $23-49 \mathrm{~mm}$ long; ventral lip reflexed, of one lobe; lobe lanceolate, $3-5 \times$ as long as wide, $12-15 \times 2.5-4$ $\mathrm{mm}$, obtuse, entire; dorsal lip erect, 3-lobed, 22-27 mm long; two lateral lobes 
spreading, obliquely triangular, about as long as wide, 7-9 $\mathrm{mm}$ long, at the base 7-10 mm wide, obtuse, entire; central lobe, the galea, $1-1+3$ as long as wide, 13-18 $\times 10-11 \mathrm{~mm}$, acute at the apex, entire. Filaments glabrous, not contorted, all connate at the base, inserted on the base of the corolla; anthers rectangular, about $1.5 \times$ as long as wide, all coherent, covered by the galea. Staminode none. Ovary appressed-pubescent or glabrous, ovoid, 3-3.5 × 1.5-2.5 mm; style exserted, as long as the corolla, pubescent above, glabrous below, apically curved along the inside of the galea, deciduous; stigma bilobed. Disk gland one, dorsal, large, emarginate, glabrous. Placentae broad, ovuliferous on the inner surface only. Berry white, globose. Seeds embedded in the fruit, dark brown, ellipsoid, 3-4 $\times$ as long as wide, $1.2-1.5 \times 0.3-0.5 \mathrm{~mm}$, longitudinally striate.

Distribution: West Indies, Colombia, Venezuela, Guiana, Trinidad and Tobago.

Ecology: In rainforests on rocks or on tree trunks; alt. $0-800 \mathrm{~m}$. Venezurla: Delta Amacuro: Caño del Guinipa, Bond, Gillin \& Brown 110 (PH, NY).

British Guiana: near Aruka, Schomburgk s.n. (not seen, cited by Schomburgk, 1848 , p. 972 , destroyed in Berlin).

French Guiana: Cayenne, Martin 100 (BM, p.p.).

\section{Drymonia Mart., Nov. Gen. 3: 57. 1829.}

Epiphytic or terrestrial shrubs, subshrubs, vines, lianas, or herbs of very diverse size, when creeping or climbing up to $5 \mathrm{~m}$ long, when erect up to $2 \mathrm{~m}$ high. Stems branched or not, mostly with spreading adventitious roots. Leaves opposite, those of a pair equal or subequal, often rather long-petiolate. Flowers axillary, usually solitary, rarely fasciculate or in pedunculate umbels. Sepals 5, green or coloured, leafy, free or nearly so, mostly large. Corolla variously coloured, infundibuliform or amply tubular, horizontal in the calyx, 1-3 $\times$ as long as the calyx, spurred at the base, ventricose or not, ampliate upwards, not contracted in the throat; limb wide, spreading, often slightly bilabiate; lobes 5 , subequal or unequal, rounded or nearly so, entire to fimbriate. Stamens 4, didynamous, included; filaments contorted or not, short-connate at the base, inserted on the base of the corolla; anthers all coherent or if not mostly connivent, oblong; cells parallel, discrete, often sagittate at the base, often partially dehiscent at the base. Ovary superior; style included; stigma bilobed, at least in the Guianan species. Disk annular or reduced to one dorsal gland. Placentae ovuliferous only on the inner or on both surfaces. Capsule globose or nearly so, bivalved; valves thick, fleshy, inside sometimes coloured. Seeds shining, ellipsoid or fusiform, obliquely striate.

Type species: $D$. calcarata Mart.

Distribution: About 35 species in tropical America.

Key to the species:

1. Stems and leaves hirsute; sepals $3-4 \times$ as long as wide, longacuminate; corolla lobes entire . . . 2. D. campostyla

Stems puberulous at the apex, minutely pubescent and shining; leaves strigillose or pubescent to glabrous; sepals $1-2 \times$ as long as wide; corolla lobes serrulate or fimbriate . . . . . 
2. Corolla lobes all serrulate or crenate-serrate; corolla practically glabrous outside ........ 1. D. calcarata Ventral corolla lobe erose-fimbriate; corolla puberulous outside . . . . . . . . . . 3. D. serrulata

1. D. calcarata Mart., Nov. Gen. 3: 58, t. 224. 1829; G. Don, Gen. Syst. 4: 653. 1838; D.C., Prod. 7: 543. 1839; Hanstein, Linnaea 26: 179,207 , 215, t. 2. f. 46. 1853; in Martius, Fl. Bras. 8 (1): 404. 1864; Linnaea 34: 355. 1865. $\quad$ Fig. 18, p. 392

Lectotype: BRAzIL: Amazonas, near R. Japura, Martius 3014 (M). Heterotypic synonym: D. buscalionii Fritsch ex Buscalioni, Annali Bot. Roma 9: 113, t. 3. 1911. Type: BraziL: Pará, near la Colonia di Annita Garibaldi, near Castanhal, Buscalioni 1765 (RO).
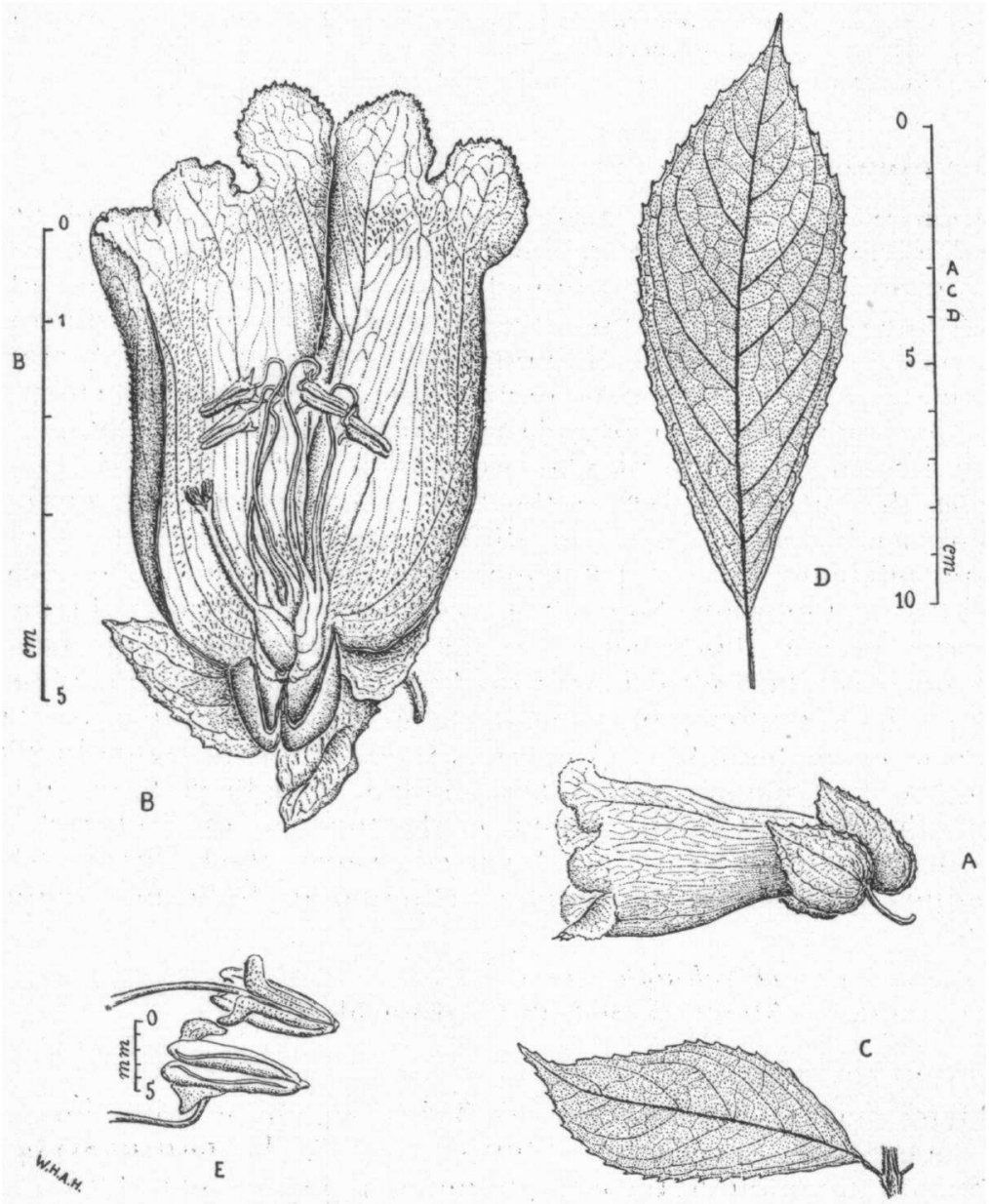

Fig. 18. Drymonia calcarata (Cowan 38630): A. flower; B. opened flower; C-D. leaves; $\mathrm{E}$. anther, both sides. 
Leafblades more or less serrulate, strigillose on both sides. Sepals green, sometimes reddish veined (teste Cowan 38607), ovate, 1.5-2 $\times$ as long as wide, acute, obtuse, or slightly acuminate at the apex, subcordate at the base, serrulate or sinuate, sparsely strigillose on both sides. Corolla $1.5-3 \times$ as long as the calyx, practically glabrous outside; limb slightly bilabiate; lobes crenate-serrate. Filaments sparsely pubescent with glandular hairs; anthers connivent, not coherent, sagittate at the base.

Vine or herb, $1 \mathrm{~m}$ high. Stems sarmentose, branched, with some spreading adventitious roots, pubescent at the apex, minutely pubescent and shining. Leaves: petiole strigillose, 5-38 mm long; blade dark green above, paler beneath, oblong, $2-3 \times$ as long as wide, $2-19 \times 1-7.5 \mathrm{~cm}$, papyraceous when dry, short-acuminate at the apex, obliquely cuneate, rounded, or subcordate at the base. Flowers solitary. Pedicel short, strigillose, $10-17 \mathrm{~mm}$ long. Sepals free, four subequal, oblique, $20-37$ $\times 10-20 \mathrm{~mm}$, the fifth (dorsal) somewhat smaller, curved around the spur. Corolla white, yellow in the throat (teste Martius) or pale yellow (teste Cowan 38607), sometimes purple-spotted (changing during anthesis?), amply tubular, $45-60 \mathrm{~mm}$ long, at the base $8-10 \mathrm{~mm}$, at the throat $20-30 \mathrm{~mm}$ wide, contracted above the spur, dorsally ventricose, ampliate towards the throat, inside partially pubescent with glandular hairs; tube $\mathbf{3 5}-\mathbf{4 0} \mathrm{mm}$ long; spur approximately globose, 6-8 $\mathrm{mm}$ in diam.; limb $25-35 \mathrm{~mm}$ wide; lobes $10-20 \mathrm{~mm}$ in diam., the ventral one larger than the two lateral ones which are slightly larger than the two dorsal ones. Filaments widened at the base; anthers glabrous, 6-7 mm long, at the apex $2 \mathrm{~mm}$, at the base $2-3 \mathrm{~mm}$ wide, spurred at the base; cells dehiscent by a longitudinal split starting at the base. No staminode seen. Ovary minutely pubescent, ovoid, 5-6 × 4-5 mm; style pubescent; stigma bilobed. Disk gland one, dorsal, entire, glabrous. Placentae ovuliferous on the inner surface only. Capsule subglobose, sparsely pubescent, $2 \times 1.5 \mathrm{~cm}$. Seeds brown, fusiform.

Distribution: northern Brazil.

Ecology: In rainforests.

Brazil: Amapá: R. Amapari, Serro do Navio, alt. 70-300 m; Cowan 38607 (NY), 38630 (US).

2. D. campostyla Lwbg., p. 305. Figs. 19, p. 394 ; 20 p. 395 Type: Suriṇame: Upper Suriname R., near Kabel, Jonker 625 (U).

Leafblades subentire or obscurely repand-serrate, hirsute or pilose on both sides, beneath especially on the midrib and veins. Sepals green, ovate-lanceolate, long-acuminate at the apex, rounded at the base, repand-serrate, hirsute on both sides, especially outside, four subequal, oblique, $3-4 \times$ as long as wide. Corolla $1.5-2 \times$ as long as the calyx, hirsute outside; lobes subequal, entire. Filaments with a single hair near the apex; anthers all coherent, not sagittate; cells bean-shaped, only apically joined, not confluent.

Creeping suffrutescent herb. Stems about $50 \mathrm{~cm}$ long, branched, with spreading adventitious roots, hirsute, especially near the apex. Leaves: petiole hirsute, $6-45 \mathrm{~mm}$ long; blade dark green above, paler beneath, often obliquely oblong-ovate or oblong-elliptic, $2-3.5 \times$ as long as wide, $2.5-10 \times 1.2-4.5 \mathrm{~cm}$, papyraceous when dry, acute or acuminate at the apex, cuneate or rounded at the base. Flowers 1-3 together. Peduncle very short or obsolete, with some bracts. Bracts lanceolate, about $5 \times$ as long as wide, 5-10 $\times 1-2 \mathrm{~mm}$, acuminate at the apex, entire, hirsute on both sides. Pedicels hirsute, 5-20 mm long. Sepals free, four 20-35 $\times 5-11 \mathrm{~mm}$, the fifth (dorsal) about half as long as the others, 3-6 $\times$ as long as wide, 12-21 $\times$ 2.5-5 mm. Corolla. white, inside yellow and purple-lined (teste Jonker), amply tubular, 35-60 mm long, at the base 3-5 mm, at the throat $10-15 \mathrm{~mm}$ wide, 
slightly contracted above the spur, not or hardly ventricose, inside partially pubescent with glandular hairs; tube $30-45 \mathrm{~mm}$ long; spur obtuse, about as long as wide, 3-5 mm long; limb 2-3.5 $\mathrm{cm}$ wide; lobes rounded, 6-13 $\mathrm{mm}$ in diam. Filaments contorted; anthers $2.5 \times 2 \mathrm{~mm}$; connective pilose at the base; cells partially dehiscent at the base. Staminode very small, $1 \mathrm{~mm}$ long, glabrous, with a triangular acuminate sterile anther. Ovary hirsute, ovoid, 4-5 $\times 2.5-3 \mathrm{~mm}$; style shortly hirsute with often obscurely aggregated hairs; stigma bilobed. Disk gland one, dorsal, large, emarginate, glabrous. Placentae ovuliferous on both surfaces. Fruit (almost mature), included in the calyx, globose, hirsute, 9-10 $\mathrm{mm}$ in diam. Seeds dark brown, ellipsoid, about $4 \times$ as long as wide, $1-1.2 \times 0.25-0.3 \mathrm{~mm}$.

Distribution: Suriname and French Guiana.

Ecology: In rainforests, at low elevations.

Suriname: Upper Suriname R., near Kabel, Jonker 625 (U, type); Abontjeman, Sara Ck., Suriname R., coll. indig. 279 (U); Emmaketen, B. W. 5642 (U).

French Guiana: Marowijne R., Wachenheim 173 (P); ibid., vic. of Codebert, Wachenheim 162 (P); Lower Mana R., Charvein, Benoist 497 (P).

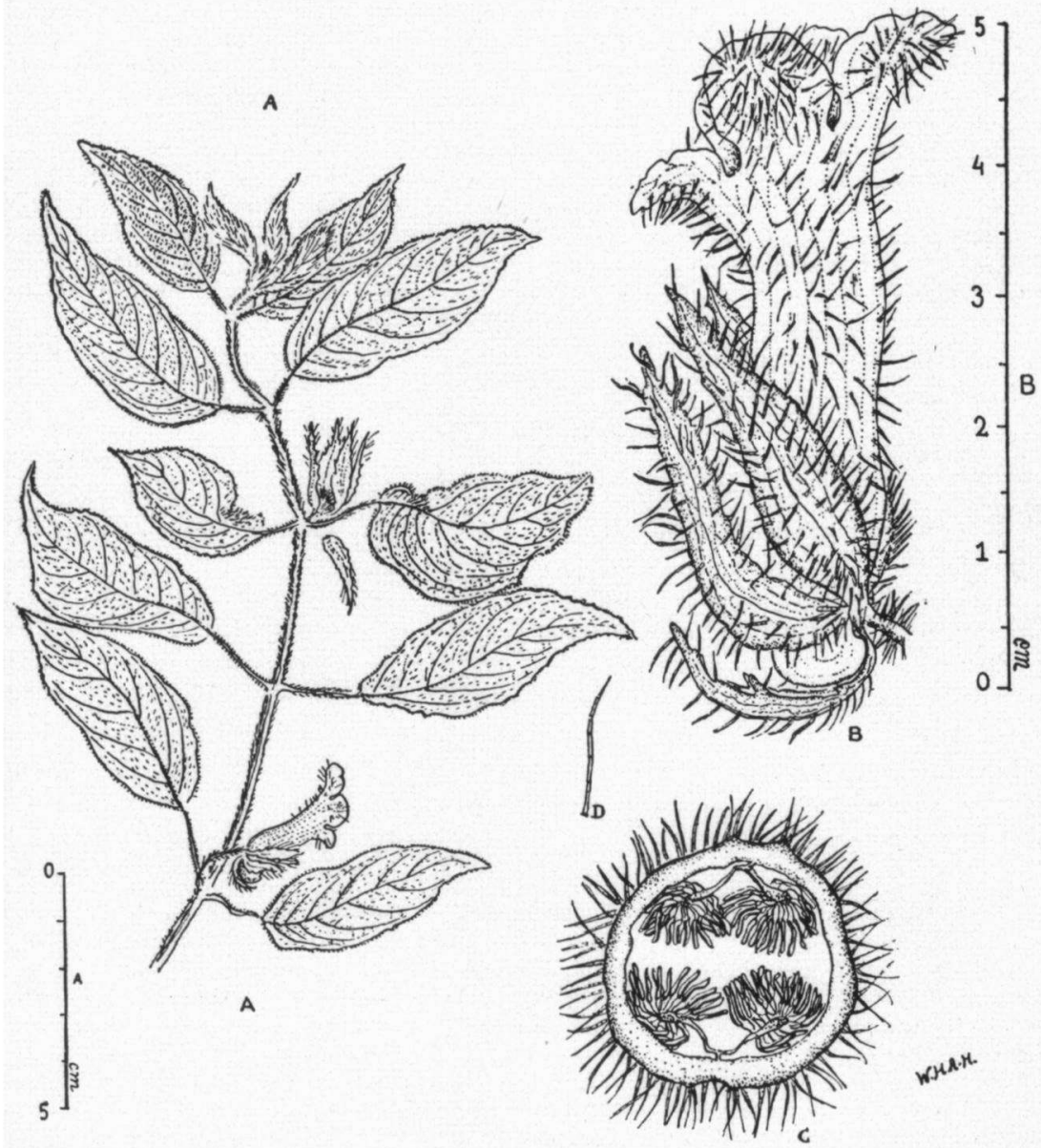

Fig. 19. Drymonia campostyla: A. branch; B. flower (A-B: B. W. 5642); C. transverse section of ovary; D. hair (C-D: Benoist 497). 
3. D. serrulata (Jacq.) Mart. ex D.C., Prod. 7: 543. 1839 (see also Martius, Nov. Gen. 3: 59. 1829); Hanstein, Linnaea 26: 179, 207. 1853; op. cit. 34: 354. 1865; Urban, Symb. Ant. 2: 355. 1901; Pulle, Enum. 420. 1906; Johnston, Contrib. Gray Herb. 34 (7): 260. 1909; Sandwith, Kew Bull. 1938: 371; Morton in Fl. Trin. and Tob. 2 (5): 309. 1955.

Fig. 21, p. 396

Basionym: Besleria serrulata Jacq., Hort. Schönbr. 3: 21, t. 290. 1798.

Type: Cult. in Hort. Schönbrunn, Jacquin s.n. (W, ster.).

Homotypic synonyms: Drymonia bicolor Lindl., Bot. Reg. 24: t. 4. 1838; Otto, Allg. Gartenz. 6: 158, t. 4. 1838.

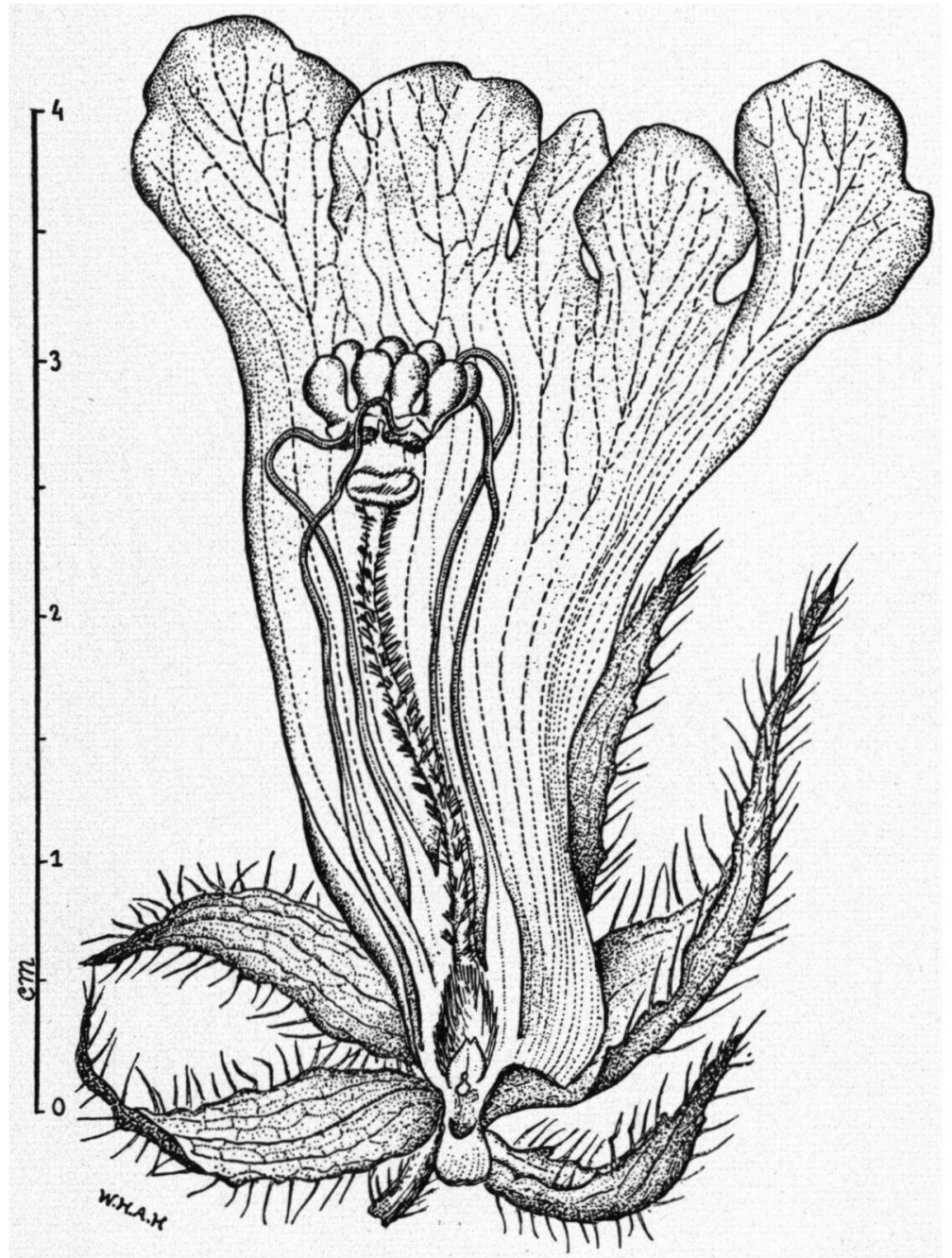

Fig. 20. Drymonia campostyla (Benoist 497), opened flower. 
Drymonia jacquini G. Don, Gen. Syst. 4: 653. 1838.

Heterotypic synonyms: Besleria spectabilis H.B.K., Nov. Gen. 2: 321. 1818 ("1817"). Type: Colombia: Cauca, Smitha R., between La Sequia and Popayan, Humboldt \& Bonpland 2028 (P-BO). Homotypic synonym: Drymonia spectabilis (H.B.K.) Mart. ex G. Don, l.c. (see also Martius, l.c.); D.C., l.c.; Hanstein, Linnaea 26: 179. 1853; op. cit. 34: 355. 1865; Oersted, Centralamer. Gesn. 37. 1858; Morton, Field Mus. Publ. Bot. 18; 1175. 1938; Johnston, Sargentia 8: 276. 1949.

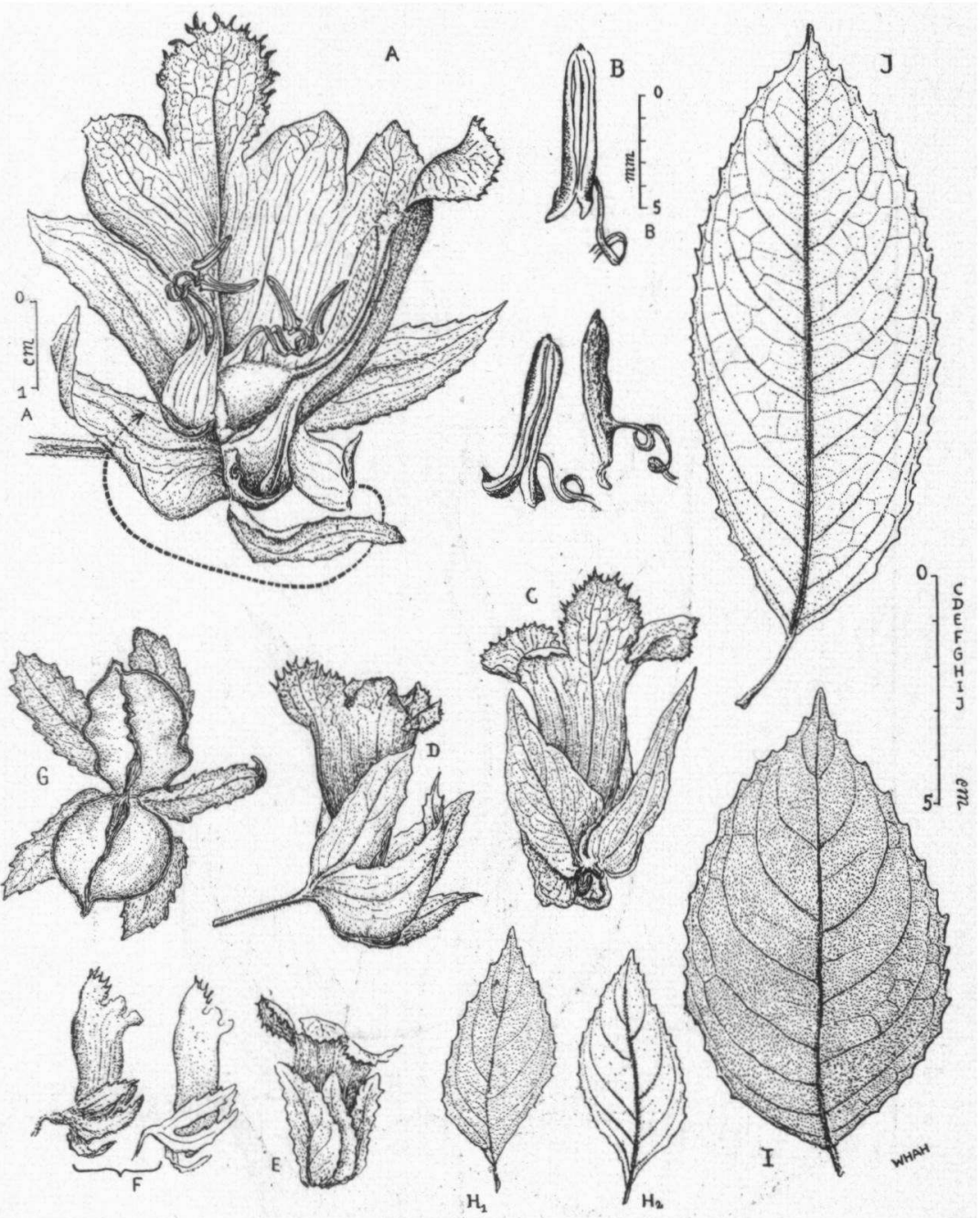

Fig. 21. Drymonia serrulata: A. opened flower; B. anthers; C-F. flowers; G. open capsule; $H_{1}$ and $I$. leaves, upper side; $H_{2}$ and $J$. leaves lower side (A, $B, D$ : Broadway, 15 Sept. 1918; C, H: von Tuerckheim II 114; E, G, J: Splitgerber 586; F: Kegel, cult. at Gent, anno 1846; I: Britton \& Broadway 2811). 
Drymonia cristata Miq., Linnaea 18: 26. 1844; Lemaire, Fl. d. Serres Sér. 1. 4: pl. 388. 1848; Lindley and Paxton, Flower Garden 1: 41, 42. f. 25. 1850; Hanstein, Linnaea 34: 356. 1865; Pulle, l.c.; Morton, Bull. Torr. Bot. Cl. 75. 1948; Lemée, Fl. Guian. fr. 3: 460. 1953. Type: SuRiname: Focke 766 (U, flowers lost).

Homotypic synonym: D. sarmentosula Lem., l.c.

D. parvifolia Griseb., Fl. Br. W.I. Isl. 463. 1861 ("1864"); Urban, l.c., not Morton. Type: Colombia: Magdalena, Santa Marta (not Jamaica !), Purdie 177 (GOET, holotype, isotype: $\mathrm{K}$, photographs in $\mathrm{U}, \mathrm{WAG}$ ).

D. maculata S. Moore, Trans. Linn. Soc. Ser. 2. 4: 410. 1895. Type: BraziL: Matto Grosso, Barro do Bugres, S. Moore 529 (BM).

Leafblades móre or less serrulate, sparingly strigillose on both sides, beneath sometimes practically glabrous between the veins. Sepals green, sometimes purple-spotted, acute or acuminate at the apex, entire, sinuate, or serrulate, puberulous or sparsely appressed-pubescent on both sides, four subequal, oblique, decurved, broadly ovate to ovate-oblong, 1.2-4 (mostly 1.5-2) $\times$ as long as wide, rounded or subcordate at the base. Corolla $1-2 \times$ as long as the calyx, puberulous outside; limb bilabiate, with two dorsal and three ventral lobes, the anterior one erose-fimbriate, larger than the others, the others serrulate. Filaments glabrous; anthers connivent, not coherent, sagittate at the base.

. Shrub, subshrub, or vine, when creeping or climbing up to $5 \mathrm{~m}$ long, when erect up to $2 \mathrm{~m}$ high. Stems sarmentose, branched or not, with spreading adventitious roots, pubescent at the apex, minutely pubescent and shining. Leaves: petiole appressed-pubescent or puberulous, 6-50 (mostly 10-20) $\mathrm{mm}$ long; blade medium or dark green above, paler green or sometimes purple beneath, elliptic or oblongelliptic, variable, $1.5-3$ (mostly $2-2.5$ ) $\times$ as long as wide, $4-19 \times 2-8 \mathrm{~cm}$ (mostly $6-10 \times 3-5 \mathrm{~cm}$ ), papyraceous when dry, acute or acuminate at the apex, cuneate to rounded at the base. Flowers mostly solitary, sometimes 3 together. Pedicels appressed-pubescent or puberulous, 5-25 mm long. Sepals free, four erect, 18-50 $\times$ 5-33 mm (mostly 20-40 $\times 15-25 \mathrm{~mm}$ ), the fifth (dorsal) smaller than the others, ovate to lanceolate, curved around the spur. Corolla creamy, pale yellow, pinkish, pink, purple- or dark red-veined or -spotted, dark red, or purple (changing during anthesis from pink to purple (teste Johnston 62, see p. 307)), 3-5.5 cm long, at the base $3-8 \mathrm{~mm}$, at the throat $10-20 \mathrm{~mm}$ wide, slightly contracted above the spur, dorsally often ventricose, ampliate towards the throat, inside partially pubescent with glandular hairs; tube $20-30 \mathrm{~mm}$ long; spur approximately globose, $5-10 \mathrm{~mm}$ in diam.; limb 14-35 mm wide, lobes rounded, about $1-1.5 \mathrm{~cm}$ in diam. Filaments contorted, widened at the base; anthers $6-7 \times 2 \mathrm{~mm}$, spurred at the base; cells dehiscent by a longitudinal split starting at the base. Staminode one, very small, glabrous, 0.5-1 mm long, without anther. Ovary puberulous, ovoid, 5-8 $\times$ 4-6 $\mathrm{mm}$; style pubescent, upcurved; stigma bilobed. Disk annular, glabrous, dorsally or laterally widened and ventrally narrowed or reduced to one dorsal gland. Placentae ovuliferous on both surfaces. Capsule globose, puberulous or appressedpubescent, 10-20 $\times 10-18 \mathrm{~mm}$, with a thickened ring along the line of dehiscence; valves coriaceous, inside purple or dark red or orange-red, fleshy and glabrous. Seeds dark brown, obliquely ellipsoid, $3-4 \times$ as long as wide, $0.8-1 \times 0.25-0.3 \mathrm{~mm}$.

Distribution: Tropical America (From Mexico and the Lesser Antilles to Matto Grosso).

Ecology: Climbing or creeping on tree trunks, over shrubs, or 
sometimes over rocks, in rainforests, in clearings, forest-edges, or on banks of rivers or lakes; alt. 0-1300 m.

British Guiana: Barima R., Mt. Terminus, Ward \& Kortright 8795 (K).

Suriname: Corantijn R., Kaboeri Ck., B.W. 2180 (U); Avanavero Falls, Kabalebo R., Corantijn R., B.W. 4598 (U); Upper Suriname R., near Komoprati Fall, Lower Pikien Rio, Tresling 251 (U) ; Saramacca R. Headwaters, above Kwattahede, Maguire 23935 (NY, A, F, K, U); near Saramacca R., near Abontjeman, coll. indig. $278(\mathrm{U})$; Groningen, Samuels 97 (GH); Suriname R., Jodensavanne-Mapane Ck. area, Lindeman 6814 (U); Paramaribo, Went 292 (U); ibid., Wullschlägel 380 (BR, GOET); ibid., near plant. Jagtlust, Splitgerber 586 (L); ibid., near plant. Geyersvlijt, Kegel 827 (GOET); Berthoud-Coulon 478 (BM); Focke 766 (U, type of D. cristata); Hostmann $208(\mathrm{~K}), 570(\mathrm{~K})$; Weigelt s.n. (PH).

FRENCH GuianA: Acarouany R., Lower Mana R., Sagot 899 (P, K, U); Gabriel, anno 1802 (G); Martin s.n. (BM); Martin, herb. Rudge s.n. (BM); Poiteau s.n. (K); L. C. Richard s.n. (P).

4. D. turrialvae Hanst., Linnaea 34: 359. 1865; Morton, Field Mus. Publ. Bot. 18: 1176. 1938; H. E. Moore, African Violets, Gloxinias, and their relatives 196, 275, f.28A. 1957.

Type: Costa Rica: Volcán de Turrialba, Wendland 517, not seen, destroyed in Berlin?

Heterotypic synonym: D. marmorata Hook. f., Bot. Mag. 110: t.6763. 1884; Morton, Field Mus. Publ. Bot. 18: 1174. 1938. Type: Cult. Kew Gardens, 14 June $1883(\mathrm{~K})$.

According to Hooker this species occurs perhaps in Guiana. The only wild specimens the present author saw came from Costa Rica and Panama. This species differs from the Guianan species of Drymonia as follows: Leaves large, broadly elliptic, about $1+x$ as long as wide, obtuse or rounded at the apex, rounded or cordate at the base. Inflorescence several-flowered, pedunculate. Calyx purple or dark red.

7. Episcia Mart., Nov. Gen. 3: 39.1829.

Terrestrial or epiphytic herbs, sometimes suffrutescent, with or without stolons, when creeping or climbing up to $1 \mathrm{~m}$ long or more (?), when erect up to $60 \mathrm{~cm}$ high. Stems branched or not, mostly with spreading adventitious roots. Leaves opposite, those of a pair equal to strongly unequal, petiolate or occasionally subsessile; blade variously shaped, ovate or oblong-elliptic to oblong-lanceolate; smaller leaf of a pair often soon deciduous. Flowers axillary, solitary, fasciculate, or in pedunculate racemes, umbels, or cymes, short- or long-pedicellate, with or without bracts. Sepals 5 , green or coloured, free or connate at the base up to one-third of their length, acute or acuminate at the apex. Corolla variously coloured, trumpet-shaped or infundibuliform, usually oblique in the calyx, spurred at the base, contracted above the spur and often in the throat, mostly not ventricose; limb spreading; lobes 5, mostly subequal, rounded, entire to fimbriate. Stamens 4, didynamous, included or sometimes slightly exserted; filaments all connate at the base or free from each other, inserted on the corolla, usually on the base; anthers orbicular, oblong, or reniform, all coherent, coherent in pairs, or free; cells parallel or divergent at the base, discrete or confluent at the apex, partially or entirely dehiscent. Ovary superior; style included; stigma bilobed, capitate, or nearly so. Disk gland one, dorsal, large, occasionally also a small ventral or two lateral ones. Placentae ovuliferous only on the inner or on both surfaces. Fruit a capsule, bivalved. Seeds shining, ellipsoid, obliquely striate. 
Type species: $E$. reptans Mart.

Distribution: About 35 species in tropical America.

Key to the species:

1. Inflorescence racemose, with large green, purple- or redveined bracts; stems with a unilateral line of adhesive roots (like Hedera) . . . . . . . . . . . 6. E. maculata

Inflorescence if several-flowered umbellate, pedunculate or sessile, without or with small bracts . . . . . . . .

2. Flowers small; corolla about $12 \mathrm{~mm}$ long; leaves of a pair strongly unequal, all petiolate, the smaller one often deciduous; blade tomentose above . . . 1. E. bryogeton

Flowers larger, at least $15 \mathrm{~mm}$ long; leaves glabrous or hairy

3. Sepals linear, often purple, hirsute, free; leafblades oblonglanceolate, acuminate at the apex, cuneate at the base or decurrent into the petiole, hirsute on both sides, not bullate

Sepals ovate, oblong, or lanceolate, free or connate; leafblades glabrous or if oblong-lanceolate hirsute and bullate . . . . . . . . . . . . . . . .

4. Corolla crimson, about $5 \mathrm{~cm}$ long, trumpet-shaped; tube narrow, hardly widened towards the throat; inflorescence few-flowered . . . . . . 9. E. porphyrotricha

Corolla white or creamy, 3-4 cm long, infundibuliform; inflorescence sessile, many-flowered - . 2. E. ciliosa

5. At least four of the sepals connate at the base for one-third of their length, acute at the apex, sparsely pilose to glabrous; leaves mostly glabrous or nearly so . 4. E. densa

Sepals free or connate at the base, acuminate, if acute plant hirsute . . . . . . . . . . . . . .

6. Plant hirsute; leafblades bullate $\cdot \cdot \cdot \cdot \cdot \cdot \cdot \dot{0} \cdot \cdot \cdot$

Plant glabrous, pubescent, strigillose, or pilose; leafblades not bullate

7. Corolla red; creeping stoloniferous herbs; leaf blades mostly purple beneath and with a pale green area along the midrib; anther cells parallel .. . . . . . . . . . . .

Corolla white and/or mauve; plant with or without stolons; leaves purple or green beneath, with a pale area above or not; anther cells divergent at the base . . . . . . . .

8. Corolla tube $30-35 \mathrm{~mm}$ long, nearly straight, slightly widened, pinkish in the throat, all lobes spreading . . . . . 10. E. reptans

Corolla tube up to $25 \mathrm{~mm}$ long, suddenly decurved in the middle and from there more widened, yellow in the throat, two dorsal lobes recurved . . . . . . 3. E. cupreata

9. Corolla about $3 \times$ as long as the width of the limb, 47-70 mm long. . . . . . . . . . 5. E. hirsuta

Corolla $1-1 \frac{1}{4} \times$ as long as the width of the limb, 24-25 mm long .. . . . . . . . . . 11. E. sphalera

10. (6). Leafblades decurrent into the petiole; corolla white, 
40-60 mm long, tube 30-45 mm long 8. E. mimuloides

Leafblades not decurrent; corolla purple or crimson, 15-30 mm long, tube 12-25 mm long . . . 7. E. melittifolia

1. E. bryogeton Lwbg., p. 312.

Fig. 22, p. 400

Type: BRITISH GuIANA: Kurupung R., near Makreba Falls, Upper Mazaruni distr., Pinkus 12 (NY, holotype, photographs: U, WAG, isotype: US).

Leaves of a pair strongly unequal, the larger one $3-4 \times$ as long as the other; blade oblong-obovate or oblanceolate, variable, acute

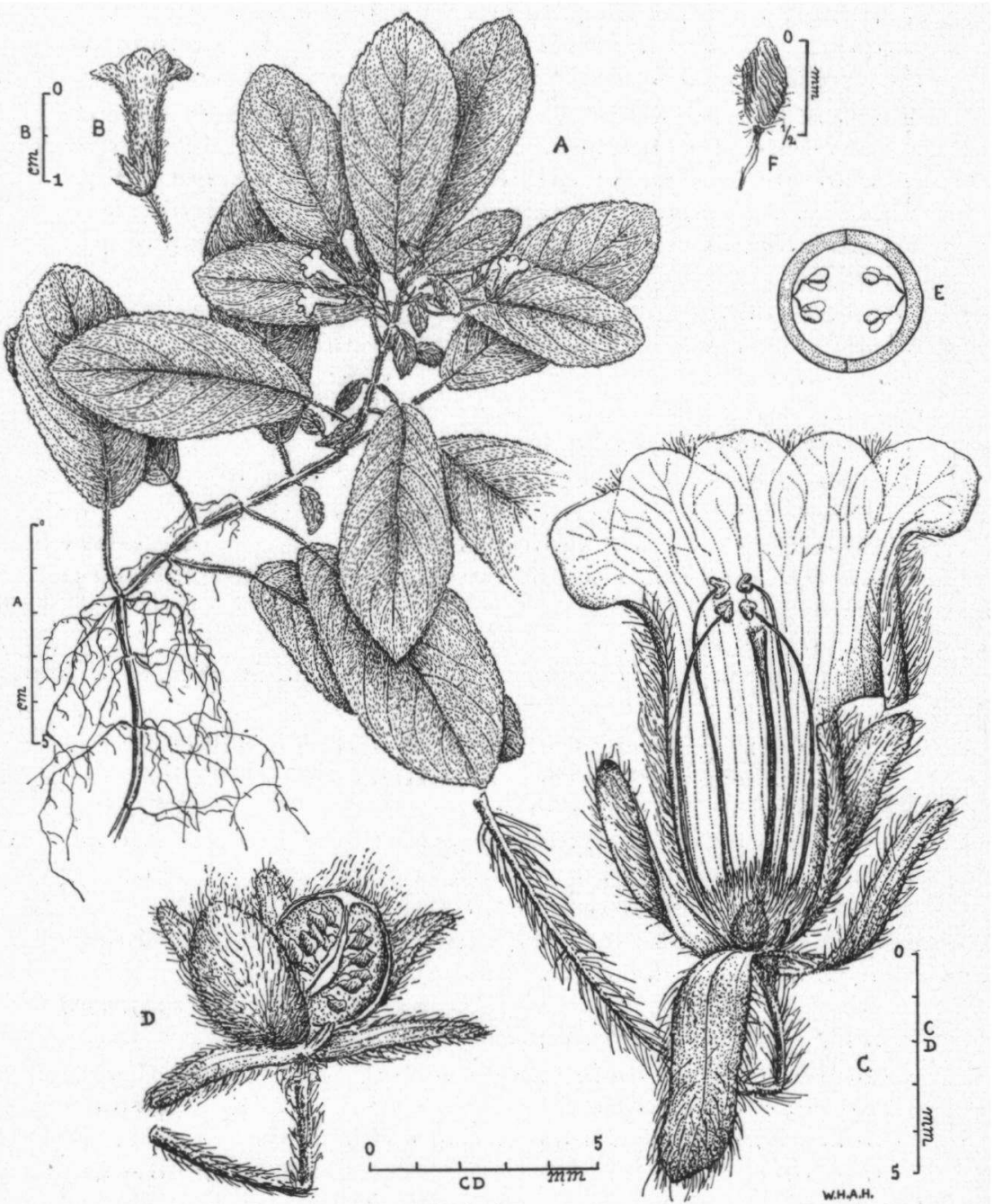

Fig. 22. Episcia bryogeton: A. branch; B. flower; C. opened flower; D. open capsule; E. diagrammatic transverse section of ovary; $F$. seed with funiculus (A-B: Pinkus 12, C-D: Altson 371). 
or obtuse at the apex, rounded or subcordate at the base, crenateserrate, tomentose above; the smaller one soon deciduous, similarly shaped and hairy. Flowers 3-10 (?) together. Sepals free, oblonglanceolate, 4-6 6 1-1.5 mm, acute at the apex. Corolla white, dorsally purple in the throat, infundibuliform, $10-14 \mathrm{~mm}$ long; villose outside. Anthers reniform.

Terrestrial creeping suffrutescent herb. Stems 2-50 (?) cm long, with often very short branches, 'with spreading adventitious roots, strigillose at the apex, woody and glabrescent towards the base. Leaves: larger one: petiole villose, 4-40 (mostly about 10-25) mm long; blade green, 1.5-2.7 $\times$ as long as wide, 32-95 $\times 17-45 \mathrm{~mm}$, papyraceous when dry, narrowed towards the base, strigillose beneath, especially on the midrib and veins; smaller one: petiole $6-7 \mathrm{~mm}$ long, blade about $2 \times$ as long as wide, 12-16 $\times 6-8 \mathrm{~mm}$. Inflorescence: bracts lanceolate, $5-7 \times 0.5-1 \mathrm{~mm}$, obscurely serrulate near the apex, villose. Pedicels slender, villose, 6-17 mm long. Sepals green, narrowed towards the base, with some teeth, villose outside, inside villose above, glabrous below, four subequal, erect, $3 \frac{1}{2}-4 \frac{1}{2} \times$ as long as wide, the fifth (dorsal) somewhat smaller and narrower, about $6 \times$ as long as wide, curved around the spur. Corolla oblique in the calyx, $2-3 \frac{1}{2} \times$ as long as the calyx, at the base $1.5 \mathrm{~mm}$, at the throat $4 \mathrm{~mm}$ wide, shortly spurred at the base, slightly contracted at $5 \mathrm{~mm}$ from the base, gradually widened to the throat, not ventricose, slightly bent downwards, glabrous inside; tube 8-11 mm long; limb 10-11 mm wide; lobes subequal, rounded, entire, $3-4, \mathrm{~mm}$ in diam. Stamens included; filaments glabrous, contorted or not, free from each other; inserted on the base of the corolla; anthers all coherent or coherent in pairs; cells confluent at the apex, dehiscent throughout by a longitudinal split. Staminode none. Ovary softly hirsute, globose, $1 \mathrm{~mm}$ in diam.; style slender, glabrous, about as long as the stamens; stigma stomatomorphic. Disk gland tongue-shaped, glabrous. Placentae ovuliferous on the inner surface only. Capsule globose, pubescent, $3 \mathrm{~mm}$ in diam. Seeds dark brown, rather obscurely and sparingly pilose, about $2 \times$ as long as wide, $0.5-0.7 \times 0.25-0.3 \mathrm{~mm}$.

Distribution: British Guiana.

Ecology: On moss-covered rocks, on slopes, at low elevations.

Britrsh Gutana: Kurupung Mts., Altson, 371 (K, 2 sheets, paratypes); ibid., Makreba Falls, Pinkus 12 (NY, US, type); Pakaraima Mts., Membaru-Kurupung Trail, Maguire \& Fanshawe 32392 (US).

2. E. ciliosa (Mart.) Hanst. in Martius, Fl. Bras. 8 (1): 403. 1864; Linnaea 34: 347. 1865; Morton, Bull. Torr. Bot. Cl. 75: 565. 1948.

Fig. 23, p. 402

Basionym: Hypocyrta ciliosa Mart., Nov. Gen. 3: 53. 1829; G. Don, Gen. Syst. 4: 652. 1838; D.C., Prod. 7: 541. 1839.

Type: BRAzIl: Amazonas, R. Japura, Manacurú, Martius 3117 (M, holotype; photographs: U, US, WAG).

Homotypic synonym: Columnea ciliosa (Mart.) Kuntze, Rev. 2: 471. 1891 .

Leaves apparently decussate, but each node bearing one lamina, one or two on each plant (?); blade oblong-lanceolate, $2.5-4 \times$ as long as wide, $17-40 \times 4.5-11 \mathrm{~cm}$, acuminate at the apex, long-decurrent into the petiole. Flowers numerous, aggregated. Sepals purplish or crimson, linear, free, hirsute outside. Corolla white or creamy, infundibuliform, 3-4 cm long, pubescent outside; lobes fimbriate. Anther cells discrete, bean-shaped, with tufts of stiff hyalinous hairs.

Epiphytic or terrestrial herb, about $30-40 \mathrm{~cm}$ high. Stems short, procumbent, with spreading adventitious roots, hirsute, glabrescent towards the base. Leaves: petiole hirsute, 6-14 mm, semiterete (teste Martius); blade dark green (?) above, 
paler beneath, chartaceous when dry, repand-serrate, ciliate, hirsute on both sides, beneath especially on the midrib and veins. Inforescence: bracts linear, shaped and coloured like the sepals, about $10 \times 1 \mathrm{~mm}$. Pedicels hirsute, $1-2.5 \mathrm{~cm}$ long. Sepals $10-15 \times$ as long as wide, 10-20 $\times 1-2 \mathrm{~mm}$, long-acuminate, with some teeth, glabrous inside, four erect, the fifth (dorsal) curved around the spur. Corolla about $2 \times$ as long as the calyx, at the base $1.5-2 \mathrm{~mm}$, at the throat $5-8 \mathrm{~mm}$ wide, shortly spurred at the base, somewhat contracted above the spur and in the throat, gradually widened, not or hardly ventricose, glabrous inside; tube about 20-33 $\mathrm{mm}$ long; spur obtuse, about $2 \mathrm{~mm}$ long and wide; lobes unequal, broadly rounded, 3-7 $\times$ 3-4 $\mathrm{mm}$ (?). Stamens included; filaments purplish, glabrous, contorted, all connate at the base for $5 \mathrm{~mm}$, inserted on the base of the corolla; anthers white, coherent in pairs; cells divergent at the base, dehiscent throughout by a longi-

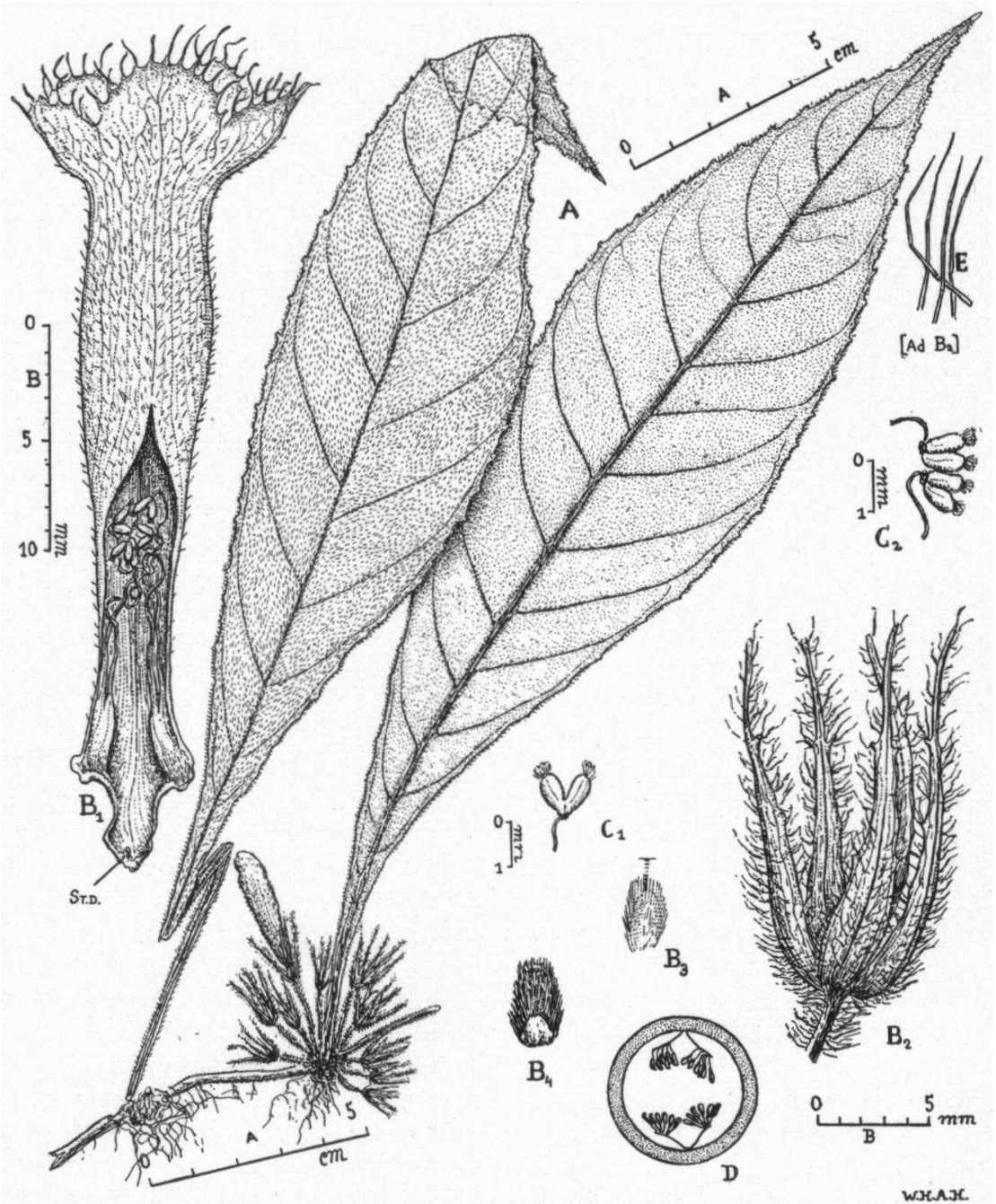

Fig. 23. Episcia ciliosa (Maguire \& Fanshawe 23019): A. plant; $B_{1}$. opened corolla; $\mathbf{B}_{2}$. calyx; $\mathbf{B}_{3}$. ovary; $\mathbf{B}_{4}$. ovary with disk; $\mathrm{C}$. anthers; D. diagrammatic transverse section of ovary; E. hairs of calyx. 
tudinal split. Staminode very small, glabrous, about $0.4 \mathrm{~mm}$ long, with a triangular sterile anther. Ovary reddish, softly hirsute, ovoid, 3-4 $\times 2-2.5 \mathrm{~mm}$; style sparsely pubescent; stigma capitate (concave, teste Martius). Disk gland tongue-shaped, glabrous, about $1.5 \mathrm{~mm}$ high. Placentae ovuliferous on the inner surface only. Fruit and seeds not seen.

Ecology: In rainforests.

Besides the type collection only known from the following:

British Guiana: Amatuk Portage, Maguire \& Fanshawe 23019 (NY, K, US); Pakaraima Mts., Membaru-Kupurung Trail, Maguire \& Fanshawe 32368 (US).

3. E. cupreata (Hook.) Hanst., Linnaea 34: 340. 1865; H. E. Moore, Baileya 1: 21, 46, f. 36A. 1953; op. cit. 2: 75. 1954; African Violets, Gloxinias, and their relatives 185, 275, 301, f. $26 \mathrm{~A} .1957$.

Basionym: Achimenes cupreata Hook., Bot. Mag. 73: t. 4312. 1847; Lemaire, Ann. Soc. Agr. Gand 3: 367, t. 156. 1847.

Type: Colombia: Magdalena (?), near Sona, Purdie 470 (K, holotype, photographs: U, US, WAG). Homotypic synonym: Cyrtodeira cupreata (Hook.) Hanst., Linnaea 26: 207,215 , t. 2. f. 39. 1853.

Creeping terrestrial herb with stolons. Leaves of very variable size, those of a pair equal or subequal; blade usually bullate, elliptic, oblong-ovate, or rounded, acute, obtuse, or sometimes acuminate at the apex, often obliquely rounded or subcordate at the base, crenateserrate, hirsute on both sides. Flowers solitary or rarely 2-3 together. Sepals lanceolate, acute at the apex. Corolla scarlet, the limb deeper than the tube, with a yellow ring and sometimes four purple spots in the throat, $20-35 \mathrm{~mm}$ long, slightly widened in the basal half, suddenly curved in the middle and from there ventrally more widened; tube 15-25 mm long; two dorsal lobes generally bent backwards.

Plant about 5-20 cm high. Stems terete, branched, with spreading adventitious roots, hirsute. Stolons hirsute, $6-50 \mathrm{~cm}$ long. Leaves: petiole hirsute, $0.5-7 \mathrm{~cm}$ long; blade $1-2 \times$ as long as wide, $13-85 \times 10-55 \mathrm{~mm}$, in young plants on stolons often smaller, membranaceous or papyraceous when dry; midrib and veins impressed above, prominent beneath; secondary veins reticulate. Inflorescence: pedicel hirsute, $1-4.5 \mathrm{~cm}$ long. Sepals green, subequal, somewhat connate at the base, $3-6 \times$ as long as wide, 5-12 × 1-3 mm, slightly narrowed towards the base, more or less serrate near the apex, hirsute on both sides, four erect, the fifth (dorsal) somewhat smaller, curved around the spur. Corolla oblique in the calyx, $2 \frac{1}{2}-5 \frac{1}{2} \times$ as long as the calyx, at the base $3-4 \mathrm{~mm}$, at the throat $6-9 \mathrm{~mm}$ wide, slightly contracted above the spur, hardly so in the throat, villose outside, inside with a ring of glandular hairs in the throat; spur obtuse, about as long as wide; limb slightly oblique on the tube, $14-25 \mathrm{~mm}$ wide; lobes subequal, rounded, crenateserrate, $6-11 \mathrm{~mm}$ in diam. Stamens included; filaments glabrous, contorted after the pollen is shed, all connate at the base, inserted on the base of the corolla; anthers all coherent, orbicular or oblong, $1-1 \frac{1}{2} \times$ as long as wide; cells parallel, discrete, dehiscent throughout by a longitudinal split. Staminode small, glabrous, $0.5-1 \mathrm{~mm}$ long, with a sterile anther. Ovary hirsute, ovoid, $2.5-4 \times 1.5-3 \mathrm{~mm}$; style glabrous or with scattered hairs near the apex; stigma capitate or obscurely bilobed. Disk gland entire or obscurely bilobed, glabrous, occasionally two tongueshaped lateral glands (Steyermark 56347). Placentae ovuliferous on both surfaces. Capsule globose, hirsute, $6 \mathrm{~mm}$ in diam. Seeds dark brown, ellipsoid, $1 \frac{1}{2}-2 \times$ as long as wide, $0.3-0.4 \times 0.15-0.25 \mathrm{~mm}$.

Distribution: Colombia, Venezuela, Brazil (Amapá).

Ecology: In shady moist places, in rainforests, mostly in the mountains; alt. 0-2400 m. 
In Guiana only one variety:

E. c. var. cupreata.

Leaf blade bullate, dark green with a pale green area along the midrib above and purple beneath.

Brazil: Amapá: Calçoene (= Carsevenne), Geay, anno 1898 (P); R. Amapari, Serro do Navio, Cowan 38562 (NY).

4. E. densa C. H. Wright, Kew Bull. 1895: 17; Hooker, Bot. Mag. 122: t. 7481. 1896; Morton, Bull. Torr. Bot. Cl. 75: 566. 1948.

Type: British Guiana: Macouria R., right bank Lower Essequibo R., Jenman 2414 ( $K$, holotype, photographs: U, US).

Homotypic synonym: Centrosolenia densa (C. H. Wright) Sprague, Kew Bull. 1912: 87; Sandwith, Kew Bull. 1931: 489.

Leaves of a pair equal or subequal, long-petiolate; blade dark green above, purple or crimson beneath, oblong-elliptic or oblong-lanceolate, variable, acute, acuminate, or sometimes obtuse at the apex, cuneate, rounded, or sometimes obliquely subcordate at the base, subentire to crenate-serrate, mostly glabrous or nearly so on both sides, sometimes strigose to subtomentose above and sparsely pilose beneath, especially on the midrib and veins. Inflorescence umbellate or subcymose. Flowers mostly numerous, aggregated. Peduncle short. Sepals purple outside, green inside, acute, four connate to one-third of their length, obliquely oblong-lanceolate, the fifth (dorsal) mostly nearly free, lanceolate. Corolla white or pale stramineous (teste Hooker), sometimes with purple or purple-tipped lobes, approximately trumpetshaped, 3-6 (mostly 4-6) cm long; tube 25-47 mm long.

Terrestrial herb, 30-60 cm high. Stems ascending, short, about $10-20 \mathrm{~cm}$ long or more (?), sometimes branched, fleshy, terete, with spreading adventitious roots, sometimes puberulous at the apex, glabrous. Leaves: petiole purple, glabrous, 1-14 (mostly 6-12) cm long; blade $1 \frac{1}{2}-4$ (mostly $2 \frac{1}{2}$ ) $\times$ as long as wide, thinly fleshy, chartaceous when dry. Inflorescence: peduncle minutely puberulous or glabrous, up to $1 \mathrm{~cm}$ long. Bracts linear-lanceolate, about $5 \times$ as long as wide, 5-10 $\times 1-2 \mathrm{~mm}$, acuminate, entire or subentire (?), practically glabrous. Pedicels purple, practically glabrous, $7-30 \mathrm{~mm}$ long. Sepals repand-serrate, sparsely pilose or glabrous on both sides, four erect, $2 \frac{1}{2}-4 \times$ as long as wide, $12-23 \times 3-8 \mathrm{~mm}$, the fifth (dorsal) $5-6 \times$ as long as wide, about 1 smaller than the others, $11-17 \times 2-5 \mathrm{~mm}$. Corolla oblique or horizontal in the calyx,2-4 $\times$ as long as the calyx, at the base $3-4 \mathrm{~mm}$, at the throat 5-12 mm wide, shortly spurred at the base, slightly contracted above the spur, not or hardly so in the throat, not or hardly ventricose, slightly bent downwards, hirsute outside, glabrous inside; limb 15-18 mm wide; lobes subequal, rounded, entire, 5-7 $\mathrm{mm}$ in diam. Stamens included; filaments glabrous, contorted, all connate at the base, inserted on the base of the corolla; anthers reniform, coherent in pairs; cells divergent at the base, confluent at the apex, dehiscent throughout by a longitudinal split. Staminode very small, about $0.5-1 \mathrm{~mm}$ long, glabrous, curved into the spur, without anther. Ovary hirsute, ovoid, $2-4 \times 1-2 \mathrm{~mm}$; style filiform, minutely puberulous or glabrous; stigma capitate. Disk gland entire or bilobed, glabrous, $1.5 \mathrm{~mm}$ high. Placentae ovuliferous on the inner surface only. Capsule globose or nearly so, $9 \times 9-12 \times 14 \mathrm{~mm}$, with scattered hairs; valves coriaceous. Seeds dark brown, $1.5-2 \times$ as long as wide, $1-1.2 \times 0.5-0.7 \mathrm{~mm}$.

Remark: Leaves mostly glabrous or nearly so, more hairy only in some specimens: B.W. 6981; Tutin 218, 217 (BM sheet only and this partially!). 
Distribution: Three Guianas.

Ecology: On forest floor, often in clearings, along the rivers and along the coast, on often white sandy soil; alt. 0-300 m.

Britrsh Gutana: Upper Mazaruni R.; de la Cruz 2139 (NY, F, MO, US); Issano Road, F. D., 7115 (K, U) ; Potaro R. Gorge, Waratuk Falls, Maguire \& Fanshawe 23036 (NY, US); Garraway Stream, Potaro R., Maguire \& Fanshawe 22982 (NY, F, K, U, US) ; Potaro R., 10 miles south of Potaro Landing, Hitchcock 17386 (NY, K, S, US); Tumatumari, Gleason 58 (NY), 83 (NY, US); BarticaPotaro Road, c. 83 miles, Tutin 217 (BM, K, U, US), 218 (BM); Moraballi Ck., Essequibo R., F. D. 2988 (K, S); ibid., near Bartica, Sandwith 49 (K, NY); Macouria $R$., right bank Lower Essequibo $R$., Jenman 2414 (K, holotype); Rockstone, Gleason 659 (NY), 660 (NY), 661 (NY); East of Ituni, south of Mackenzie, Cowan 39228 (US).

Suriname: Wilhelmina Mts., B.W. 6981 (U)

French Guiana: Cayenne, Martin s.n. (BM, P, U); between Roura and Kaw, Martin s.n., herb. Rudge (BM); Mt. Kaw, Cowan 38725 (U); anonym. (P). Cult.: Kew Gardens, from Demerara, 7 Sept. 1892 (K); Kew Gardens, from Br. Guiana, 24 Oct. 1895 ( $K$, specimen depicted in the Bot. Mag.).

5. E. hirsuta (Benth.) Hanst., Linnaea 34: 350. 1865; Morton, Bull. Torr. Bot. Cl. 75: 566. 1948. Fig. 24, p. 404

Basionym: Centrosolenia hirsuta Benth., Lond. Journ. Bot. 5: 362. 1846.

Type: British Guiana: banks of Paramu R., Schomburgk s.n. (K, holotype, photographs: U, US).

Heterotypic synonym: Episcia cordata Gleason, Bull. Torr. Bot. Cl. 58: 466. 1931. Type: Venezuela: Amazonas, slopes of Mt. Duida, waterfall at Aguita, near Orinoco R., Tate 878 (NY, holotype, photographs: $\mathrm{U}, \mathrm{WAG}$; isotypes: $\mathrm{K}, \mathrm{US}$ ).

Leaves of a pair subequal to strongly unequal (on a single branch!); blade olivaceous with a pale green area along the midrib above and purplish or reddish beneath or entirely green, often obliquely oblongelliptic, variable in shape and size, bullate, acute, obtuse, or rounded at the apex, subcordate or rounded at the base, irregularly crenateserrate (larger leaves not rarely bicrenate), hirsute on both sides, beneath especially on the midrib and veins. Flowers 1-6 together. Sepals green or purplish (?), free, lanceolate, acuminate. Corolla tube white, limb pale mauve or lavender, trumpet-shaped, 47-70 mm long.

Creeping terrestrial herb, with stolons. Stems short, with spreading adventitious roots, hirsute at the apex, glabrescent. Stolons up to $75 \mathrm{~cm}$ long. Leaves: petiole more or less hirsute, $0.7-13 \mathrm{~cm}$ long; blade $1 \frac{1}{2}-2 \frac{1}{2}$ (mostly about 2) $\times$ as long as wide, $2-15 \times 1.3-8 \mathrm{~cm}$, rarely smaller, papyraceous when dry. Inflorescence: pedicels slender, hirsute, 8-50 (mostly 12-35) mm long. Sepals serrate, hirsute on both sides, especially outside, four subequal, about 5-6 $\times$ as long as wide, $6-15 \times 1.2-5$ $\mathrm{mm}$, the fifth (dorsal) somewhat smaller and narrower, 5-12 $\times 0.5-1.5 \mathrm{~mm}$, curved around the spur. Corolla oblique in the calyx, 5-10 $\times$ as long as the calyx, at the base $2.5-5 \mathrm{~mm}$, at the throat $10-15 \mathrm{~mm}$ wide, contracted above the spur and in the throat, dorsally slightly ventricose, slightly bent downwards, villose outside, glabrous inside; tube $33-50 \mathrm{~mm}$ long; spur obtuse, $2-5 \mathrm{~mm}$ long and wide; limb 15-42 $\mathrm{mm}$ wide; lobes subequal, crenate-serrate, 6-17 $\mathrm{mm}$ in diam. Stamens included; filaments glabrous, contorted, widened towards the base, all connate at the base, inserted on the base of the corolla; anthers reniform, coherent in pairs or free; cells divergent at the base, confluent at the apex or not, dehiscent throughout by a longitudinal split. Staminode very small, glabrous, about $\mathrm{mm}$ long, acuminate, without anther. Ovary softly hirsute, ovoid, $3-4 \times 1.5-2$ 
mm; style filiform, glabrous or nearly so; stigma often obscurely bilobed. Disk gland tongue-shaped or rarely rectangular, glabrous, $1.5-1.7 \mathrm{~mm}$ high. Placentae ovuliferous on the inner surface only. Capsule globose, hirsute, 7-8 $\mathrm{mm}$ in diam. Seeds dark brown, obliquely ellipsoid, about $2 \times$ as long as wide, $0.7-1 \times 0.35-0.5$ $\mathrm{mm}$, borne on long funiculi with a white arillus.

Distribution: Guayana Highlands.

Ecology: On wet rocks near waterfalls in montane rainforests, at middle and high elevations.

British Guiana: Paramu R., Schomburgk s.n. (K, holotype); Kurupung R., near Makreba Falls, Pinkus 13 (NY); ibid., Altson 321 (K, U); vic. of Mt.

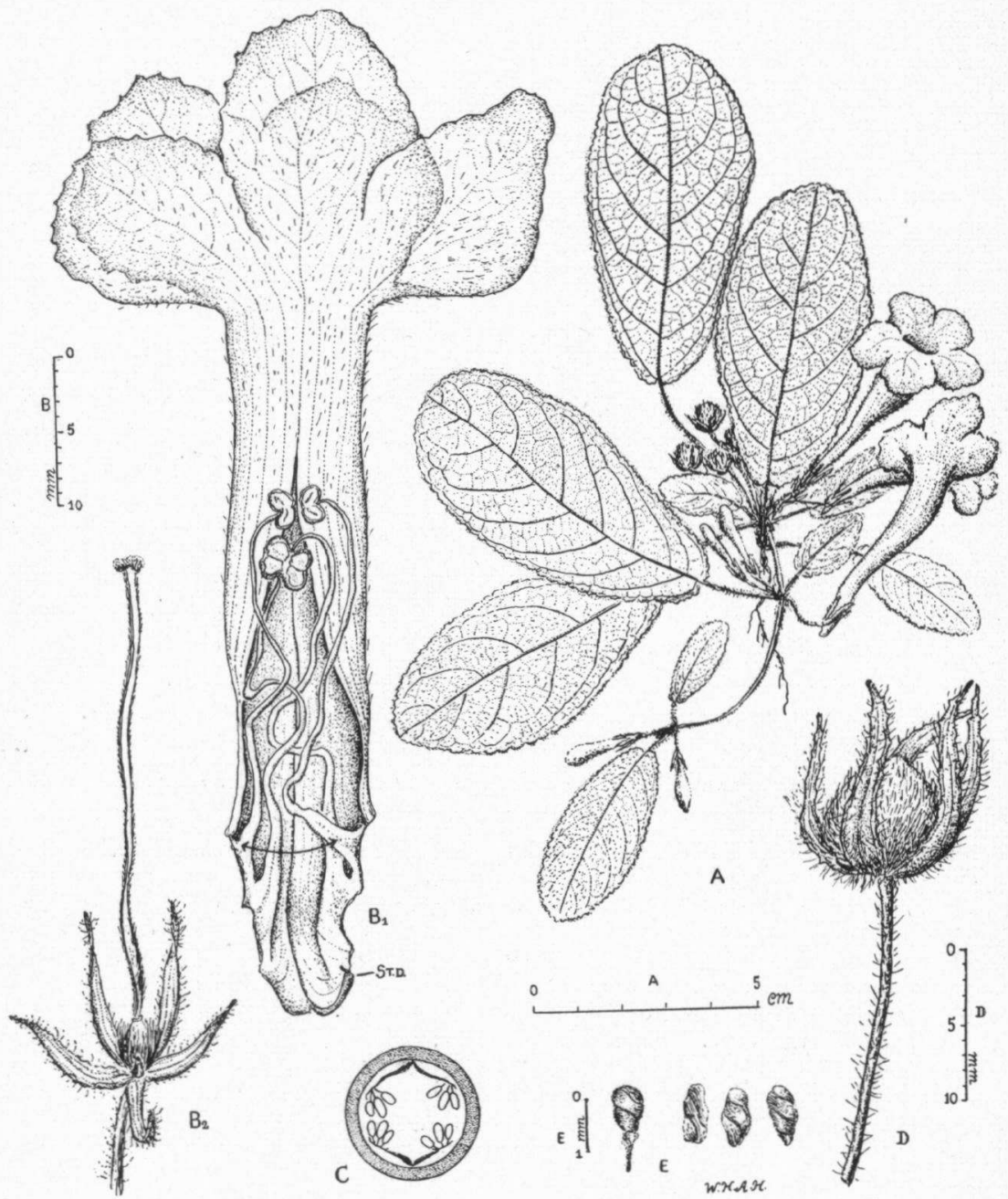

Fig. 24. Episcia hirsuta: A. plant (Appun 976); $B_{1}$. opened corolla; $B_{2}$. calyx with pistil (B: Sandwith 1279); C. diagrammatic transverse section of ovary; D. capsule (Altson 321); E. seeds, one with funiculus (Appun 976). 
Roraima, Moruakow, Abbengets 64 (K); Mt. Ayanganna, Pakaraima Mts., Maguire, Bagshaw \& Maguire 40586 (NY, US), 40588 (US); Potaro R. Gorge, Tukeit, Maguire \& Fanshawe 23078 (NY, K, US); ibid., on ascent from Tukeit, Sandwith 1279 (K); Kaieteur Savanna, Hollister, anno 1936 (NY); ibid., Jenman 1250 (K, P); Cucuyu Ck., Appun 976 (K).

6. E. maculata Hook. f., Bot. Mag. 116: t. 7131. 1890; Morton, Bull. Torr. Bot. Cl. 75: 566. 1948.

Type: Cult. Kew Gardens, 2 Sept. 1889 (K, holotype, photographs: U, US, WAG).

Stems with a unilateral line of adhesive roots. Leaves of a pair equal or subequal, long-petiolate; blade medium green above, paler green or sometimes purple beneath, elliptic or oblong-elliptic, variable, acuminate at the apex, cuneate, rounded, or occasionally subcordate at the base, sparsely pilose to glabrous above. Inflorescence racemose, up to $14 \mathrm{~cm}$ long, with large green, purple- or red-veined bracts. Sepals green, purple- or red-veined, linear-lanceolate. Corolla yellow or pale creamy (teste Archer 2432), spotted with bright red, brown, or purple-brown, infundibuliform.

Creeping or climbing vine on tree-trunks, up to $1 \mathrm{~m}$ high. Stems terete, branched or. not, hirsutulous at the apex, glabrescent. Leaves: petiole sparsely pilose to glabrous, 1.5-19 (mostly about 5-10) cm long; blade shining (teste Hooker), 1.5-3 (mostly $2-3) \times$ as long as wide, $7.5-29 \times 3.5-12 \mathrm{~cm}$, papyraceous when dry, serrate, with more or less pronounced teeth, sparsely pubescent beneath, especially on the midrib and veins; midrib and veins impressed above, prominent beneath. Flowers numerous. Peduncle short, sparsely pubescent, 1-3 cm long. Bracts leafy, shortly petiolate or subsessile, rounded, oblong, or ovate, $1-2 \frac{1}{2} \times$ as long as wide, $2-5.5 \times 1-3.5 \mathrm{~cm}$, acute or obtuse at the apex, cuneate, rounded, or subcordate at the base, serrate to subentire, sparsely appressed-pubescent to glabrous on both sides, the outer ones mostly larger than the others and mostly rounded or nearly so. Pedicels sparsely pubescent, 5-22 mm long. Sepals leafy, spreading, connate at the base, acute or acuminate at the apex, narrowed towards the base, serrate towards the apex, sparsely appressed-pubescent on both sides, four subequal, 5-8 $\times$ as long as wide, $20-40 \times 4-7 \mathrm{~mm}$, the fifth (dorsal) half as big as the others, curved around the spur, about $10 \times$ as long as wide, 9-17 $\times 1-2 \mathrm{~mm}$. Corolla oblique in the calyx, $1 \frac{1}{2}-2 \frac{1}{2}$ (mostly about 2 ) $\times$ as long as the calyx, 44-55 $\mathrm{mm}$ long, at the base $4-5 \mathrm{~mm}$, at the throat $10-18 \mathrm{~mm}$ wide, slightly contracted above the spur, not so in the throat, not ventricose, macroscopically glabrous outside, quite glabrous inside; tube $27-35 \mathrm{~mm}$ long; spur obtuse, about $2 \times$ as long as wide, $7 \times 4 \mathrm{~mm}$; lobes subequal, entire, 10-12 $\mathrm{mm}$ in diam., four spreading, the fifth (ventral) inflexed over the throat (teste Hooker) like a trap-door. Stamens included; filaments glabrous, contorted or not, free from each other, inserted somewhat under the middle of the corolla tube; anthers all coherent, orbicular or nearly so; cells parallel, discrete, dehiscent throughout by a longitudinal split. Staminode very small, glabrous, about $\$ \mathrm{~mm}$ long, without anther. Ovary pubescent, ovoid, 4-5 $\times 3-4 \mathrm{~mm}$; style filiform, about as long as the stamens, glabrous; stigma obscurely bilobed. Disk gland entire, glabrous. Placentae ovuliferous on both surfaces. Capsule subglobose, sparsely pubescent, 10-15 $\times 8$-14 $\mathrm{mm}$. Seeds medium brown, subglobose, $1.2-1.6 \times$ as long as wide, $0.7-1 \times 0.5-0.7$ $\mathrm{mm}$, rounded at the apex, mucronate at the base, borne on long funiculi with a small arillus.

Distribution: British Guiana.

Ecology: In rainforests; on sandy soil (teste F.D. 6897), at low and middle elevations.

British Guiana: Morawhanna, Bartlett 8600 (K); Matthews Ridge, Barima R., Cowan 39337 (US); Mt. Terminus, Barima R., Ward, May 1907 (K); Waini R., 
Beckett, July 1906 (K, U); Upper Aruau R., Aruka R., Martyn 53 (K); Portage between Aruau R. and Yarikita R., Hitchcock 17601 (NY, K, S, US); Pomeroon R., Jenman 2002 (K, NY); ibid., de la Cruz 3022 (NY, US), 3124 (NY, F, MO, PH, US); ibid., Yawiami Ck., Jenman 1939 (K); Pomeroon distr., Mt. Russell, Jenman 2097 (K); Kaieteur Falls, Potaro R., de la Cruz 4395 (NY, F, US); Potaro, 10 miles south of Potaro Landing, Hitchcock 17397 (NY, K, S, US); Upper Rupununi R., near Dadanawa, de la Cruz 1518 (NY, F, MO, PH, US), 1535 (NY, F, MO, PH, UC, US); Basin of Essequibo R., Haiowa Falls, A. C. Smith 2123 (NY, F, G, K, S, U, US) ; Essequibo County, near Mazaruni Forest Station, Archer 2432 (US, K); ibid., F.D. 3521 (K); Kamuni Ck., Groete Ck., Essequibo R., Maguire \& Fanshawe 22826 (NY, F, K, U, US); Rockstone, Gleason 664 (NY); Arawai Ck., right bank Essequibo R., F.D. 6897 (K, U); Macouria R., right bank Lower Essequibo R., Jenman 2419 (K).

7. E. melittifolia (L.) Mart., Nov. Gen. 3: 42. 1829; G. Don, Gen. Syst. 4: 655. 1838; D.C., Prod. 7: 547. 1839; Hooker, Bot. Mag. 79: t. 4720. 1853; Grisebach, Fl. Br. W.I. Isl. 462. 1861 ("1864"); Hanstein, Linnaea 34: 343. 1865; Duss, Fl. Ant. fr. 431. 1897; Urban, Symb. Ant. 2: 354. 1901; in Fedde, Repert. Beih. 5: 46. 1920.

Basionym: Besleria melittifolia L., Sp. Pl. 619. 1753; J. E. Smith, Exotic Bot. 1: t. 54. 1804; Schrank, Hort. Monac. 1: t. 44. 1819. Neotype: Burmann, Plant. Amer. Car. Plumier. 1756. t. 48, "Besleria pedunculis ramosis".

Homotypic synonyms: Skiophila melittifolia (L.) Hanst., Linnaea 26: 207, 215, t. 2. f. 41. 1853; Morton in Fl. Trin. and Tob. 2 (5) : 314.1955. Chrysothemis melittifolia (L.) G. Don (as melissaefolia) in Loudon, Encycl. suppl. 2: 1402. 1855.

Chrysothemis venosa Dcne., Rev. Hortic. Sér. 3. 3: 242. 1849; op. cit. 4: 381. 1850.

Heterotypic synonym: Besleria guadalupensis D.G., Prod. 7: 538. 1839. Lectotype: GUADELOUPE: l' Herminier, anno 1822 (G-DG). Homotypic synonyms: Episcia guadalupensis (D.C.) Hanst., Linnaea 34: 345.1865.

Episcia melittifolia (L.) Mart. var. guadelupensis (D.C.) Urb., Symb. Ant. 2: 354. 1901 .

Leaves of a pair equal or subequal; blade dark green and shining above, paler green and sometimes purple-veined beneath, oblongelliptic, acute at the apex, obliquely cuneate or rounded at the base, sparsely strigillose to glabrous or sometimes densely pilose to subtomentose on both sides. Inflorescence umbellate or subcymose, 1-12flowered. Peduncle often very short, with bracts. Sepals green, lanceolate or ovate-lanceolate, long-acuminate at the apex. Corolla crimson or purple, approximately trumpet-shaped, $15-30 \mathrm{~mm}$ long.

Terrestrial succulent herb (not unlike Impatiens). Stems sappy, erect or ascending, mostly unbranched, rooting at the base, terete, densely strigillose or subtomentose at the apex, glabrescent (indument rather variable). Leaves: petiole sparsely strigillose to glabrous, $1-8 \mathrm{~cm}$ long; blade $1 \frac{1}{2}-3$ (mostly about 2 ) $\times$ as long as wide, 4-27 $\times 2-13 \mathrm{~cm}$, membranaceous when dry, crenate. Inflorescence: peduncle up to $4 \mathrm{~cm}$ long. Bracts linear-lanceolate, 4-7 $\times$ as long as wide, $7-20 \times 2-5 \mathrm{~mm}$, acuminate, entire or with some teeth, sparsely strigillose on both sides. Pedicels slender, sparsely strigillose or sometimes villosulous, 1-7 (mostly about 3 ) $\mathrm{cm}$ long.

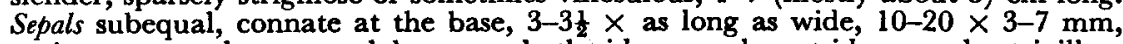
entire or repand-serrate, glabrous on both sides or only outside sparsely strigillose 
or villosulous, four erect, the fifth (dorsal) curved around the spur. Corolla oblique in the calyx, $1 \frac{1}{2}-3 \times$ as long as the calyx, at the base 3-5 mm, at the throat 5-7 mm wide, not contracted in the throat, slightly widened, not ventricose, somewhat bent downwards, strigillose outside, glabrous inside; tube 12-25 mm long; spur very short, obtuse; limb 9-15 $\mathrm{mm}$ wide; lobes subequal, entire, 4-6 $\mathrm{mm}$ in diam. Stamens included; filaments glabrous, flattened at the base, all connate at the base, inserted on the base of the corolla; anthers creamy, orbicular, coherent in pairs; cells parallel, hardly or not confluent at the apex, dehiscent throughout by a longitudinal split. Staminode very small, triangular, glabrous, about $\mathbf{m m}$ long, without anther. Ovary hirsute, ovoid, 4-5 $\times 2-2 \frac{1}{2} \mathrm{~mm}$; style included, glabrous; stigma bilobed. Disk gland bilobed, glabrous. Placentae ovuliferous only on the inner or (as figured by Hooker) on both surfaces. Capsule subglobose, strigillose, $6 \times 5-8 \times 7 \mathrm{~mm}$. Seeds dark brown, $1 \frac{1}{2}-2 \times$ as long as wide, $0.4-0.5 \times$ $0.25-0.3 \mathrm{~mm}$, obtuse or rounded at the apex, mucronate at the base.

Distribution: Lesser Antilles; Guiana?

Ecology: In rainforests, at middle elevations.

Description based on West Indian material. From Guiana only known by a specimen cited by ScHомBURGK $(1848$, p. 972$)$ which has been destroyed in Berlin, and by two collections from French Guiana the labels of which may have been exchanged.

British Gutana: Kanuku Mts., Schomburgk s.n.

French Guiana: Leblond 286 (G); herb. Maire, herb. Cosson 18 (P).

AuBlet's specimen $(1775$, p. 636$)$ is Nautilocalyx pictus.

8. E. mimuloides Benth., Lond. Journ. Bot. 5: 362. 1846; Schomburgk, Reisen Br. Guian. 3: 972. 1848; Hanstein, Linnaea 34: 345. 1865; Urban in Fedde, Repert. 17: 165. 1921 ; Graham, Ann. Carneg. Mus. 22: 250. 1934 ("1933"); Sandwith, Kew Bull. 1938: 371.

Lectotype: BRITISH GuIANA: Roraima, Schomburgk 843 (1425) (K, lectotype, isotypes: BM, G, P, 2 sheets, $W$, photographs of one $P$ sheet in $U$ and $U S$ ).

Homotypic synonym: Nautilocalyx mimuloides (Benth.) Morton in Fl. Trin. and Tob. 2 (5): 304. 1955; Leeuwenberg, Baileya 5: 23.1957. Leaves of a pair equal or subequal; blade medium green above, paler beneath, oblong, acuminate at the apex, decurrent into the petiole, sparsely pubescent above, puberulous beneath, especially on the midrib and veins. Inflorescence umbellate or subcymose, 1-8flowered. Peduncle short, with bracts. Sepals green, ovate or oblongovate, acuminate at the apex, sparsely pubescent outside. Corolla white or rose-white (teste Archer 2321), infundibuliform, 4-6 cm long.

Terrestrial herb, 15-40 cm high. Stems sappy, creeping or ascending, branched or simple, with spreading adventitious roots, puberulous at the apex, glabrescent. Leaves: petiole puberulous, $1-4.5 \mathrm{~cm}$ long; blade $2-3 \times$ as long as wide, 5-19 $\times$ $2.5-7.5 \mathrm{~cm}$, membranaceous when dry, crenate-serrate. Inflorescence: peduncle puberulous, $0.5-1.5 \mathrm{~cm}$ long. Bracts green, oblong-lanceolate, $2 \frac{1}{2}-4 \times$ as long as wide, $6-15 \times 2-5 \mathrm{~mm}$, acuminate at the apex, entire, sparsely appressed-pubescent to glabrous on both sides. Pedicels slender, puberulous to glabrous, $1-3 \mathrm{~cm}$ long. Sepals free, $2-2 \frac{1}{2} \times$ as long as wide, $10-16 \times 5-7 \mathrm{~mm}$, rounded or subcordate at the base, entire, minutely puberulous inside, four erect, oblique, the fifth (dorsal) curved around the spur. Corolla oblique in the calyx, $3-4 \times$ as long as the calyx, at the base $4-5 \mathrm{~mm}$, at the throat $9-14 \mathrm{~mm}$ wide, shortly spurred at the base, hardly contracted above the spur and in the throat, slightly widened, hardly ventricose, pilose outside, inside partially with scattered minute 2-celled glandular hairs; tube 3-4.5 cm long; spur obtuse, $2 \times 3 \mathrm{~mm}$; limb 17-26 mm wide; lobes 
subequal, entire, 4-6 mm in diam. Stamens included; filaments glabrous, contorted, free from each other, inserted on the corolla at 3-4 $\mathrm{mm}$ from the base; anthers free, oblong; cells parallel, hardly confluent at the apex, dehiscent throughout by a longitudinal split. Staminode very small, glabrous, about $\neq \mathrm{mm}$ long, without anther. Ovary hirsute, ovoid, $3-4 \times 2-3 \mathrm{~mm}$; style included, shorter than the stamens, glabrous, thickened towards the apex; stigma bilobed. Disk gland tongue-shaped, glabrous. Placentae ovuliferous on the inner surface only. Capsule globose, sparsely pubescent, $10 \mathrm{~mm}$ in diam. Seeds dark brown, $1 \frac{1}{2}-2 \times$ as long as wide, $0.6-0.8 \times 0.3-0.4 \mathrm{~mm}$, obtuse at the apex, mucronate at the base.

Distribution: British and French Guiana and Tobago.

Ecology: In dense rainforests on riverbanks on sandy soil, on ridges or on sandstone (?), at low and middle elevations.

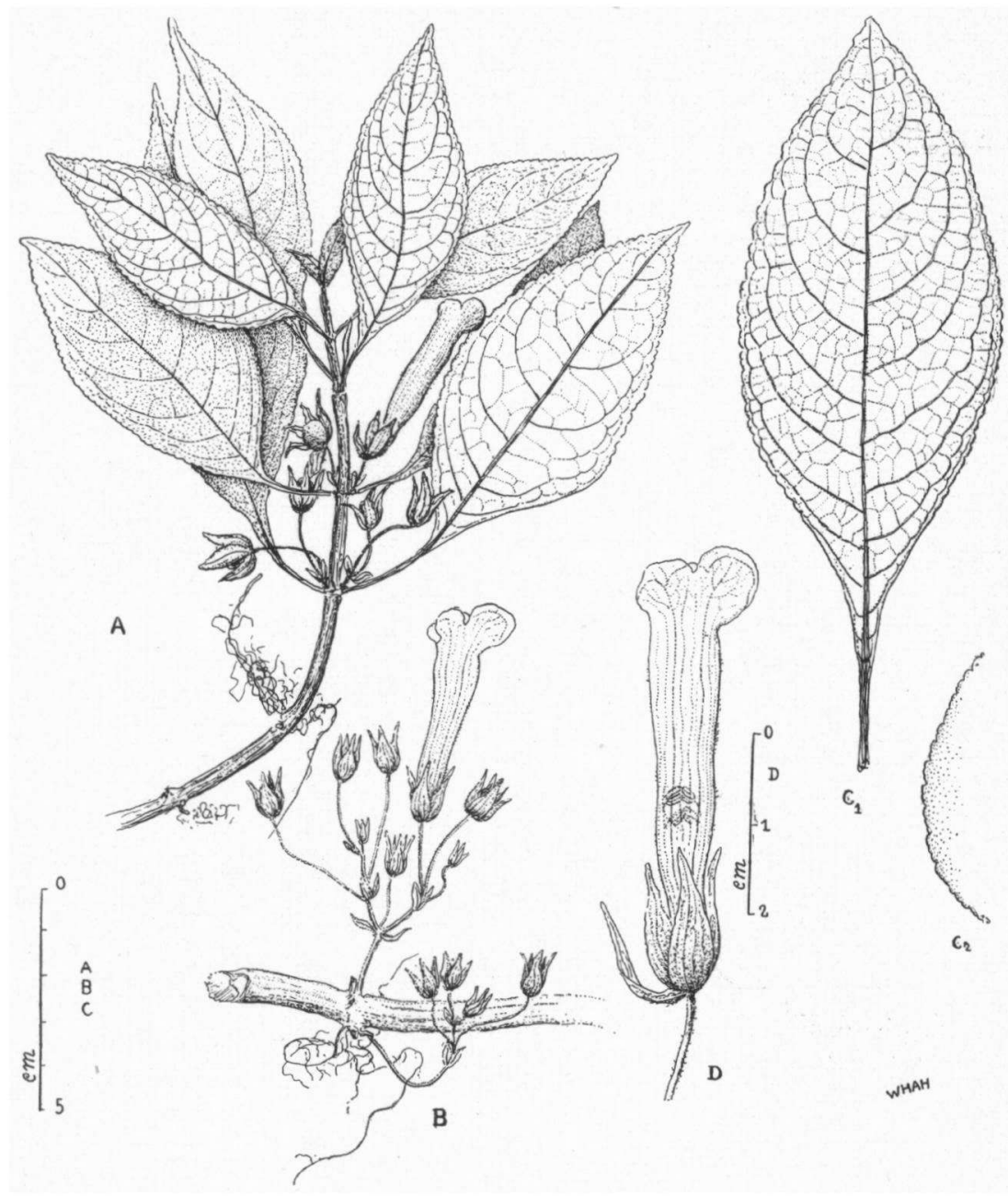

Fig. 25. Episcia mimuloides: A. plant (Schomburgk 843); B. portion of branch with inflorescences; C. leaf (B-C: Maguire a.o. 40488); D. flower (F. D. 2022). 
British Guiana: Barima R., Jenman 7123 (K, U); ibid., Matthews Ridge, Maguire 40488 (US); Portage between Aruau and Yarikita Rs., Hitchcock 17595 (NY, S, US); Arawau R., Boniska Landing, Archer 2321 (US, K); Cuyuni R., Kamaria Road, F.D. 2022 (K, F); Kamaria carry over, Foote s.n. (K); near Lower Kamaria Landing, Sandwith 659 (K, NY); near junction of Mazaruni R. and Cuyuni R., Graham 397 (NY); Roraima, Schomburgk 843 (1425) (K, lectotype, isotypes: BM, G, P, W); Schomburgk 125 (78) (K, paratype).

FRENCH Guiana: Poiteau s.n. (K, LE).

9. Episcia porphyrotricha Lwbg., p. 311 Fig. 26, p. 411 Type: British Guiana: Pakaraima Mts., Wenamu R., Davenport 7 (K, holotype, photographs: U, WAG).

Leaves of a pair unequal (?); blade dark green above, medium green (?) beneath, oblong-lanceolate, acuminate at the apex, cuneate or decurrent into the petiole, hirsute on both sides. Flowers 1-2 together. Sepals purplish-green, linear, purple-hirsute outside. Corolla

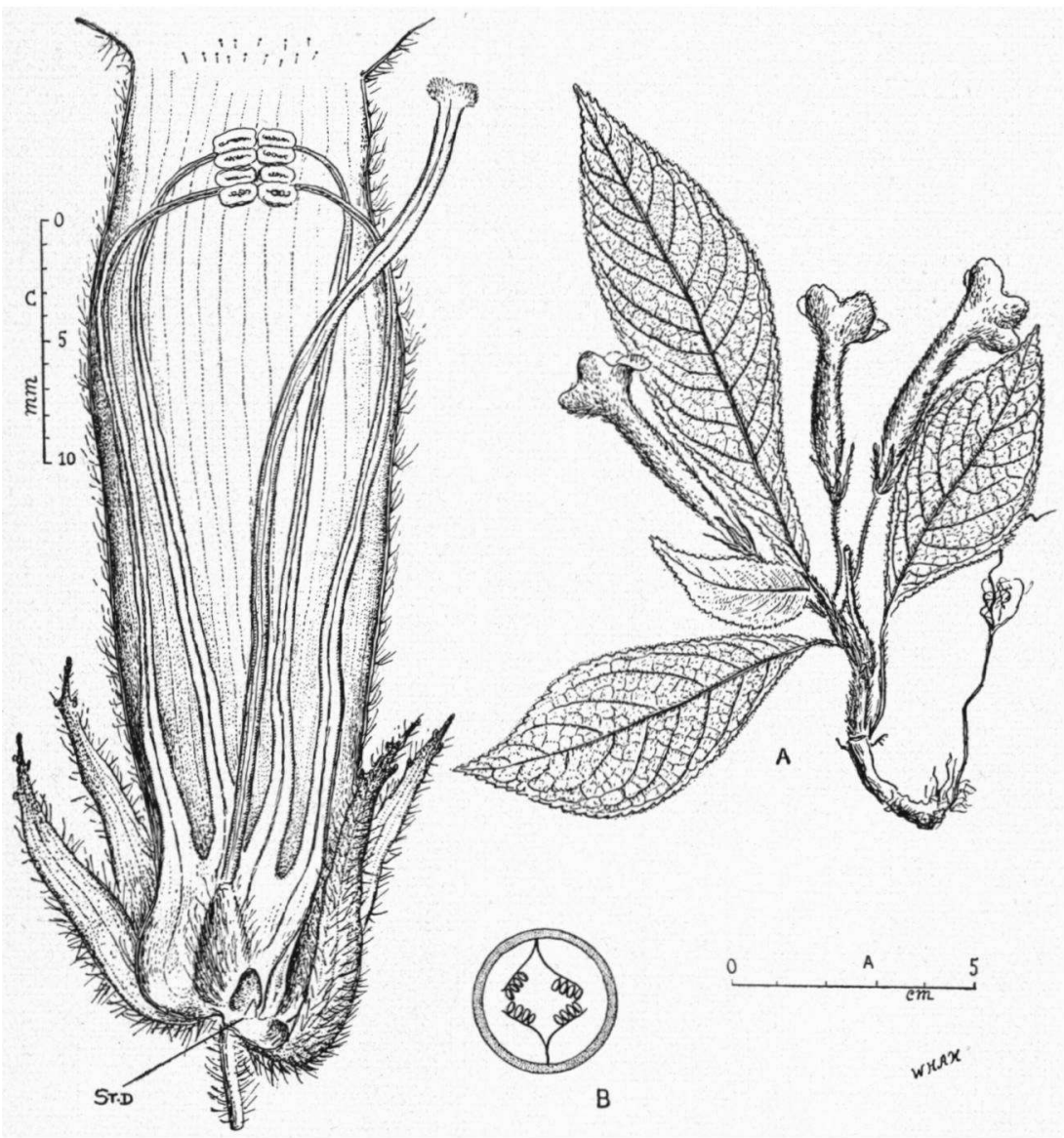

Fig. 26. Episcia porphyrotricha (Davenport 7): A. plant; B. diagrammatic transverse section of ovary; C. opened flower. 
crimson (teste Davenport), trumpet-shaped, 47-54 $\mathrm{mm}$ long; tube cylindric.

Terrestrial herb, about $15 \mathrm{~cm}$ high. Stems short, thick, with some adventitious roots, setose at the apex, glabrescent. Leaves: petiole hirsute, $8-40 \mathrm{~mm}$ long; blade $21-4 \times$ as long as wide, $7-16 \times 2.5-4 \mathrm{~cm}$, papyraceous when dry, serrate. Inflorescence: pedicels slender, purple-hirsute, $20-25 \mathrm{~mm}$ long. Sepals free, about $10 \times$ as long as wide, $10-15 \times 1-2 \mathrm{~mm}$, acuminate at the apex, with some teeth near the apex, sparsely pubescent inside, four erect, the fifth (dorsal) curved around the spur. Corolla erect in the calyx, about $4 \times$ as long as the calyx, at the base $3-4 \mathrm{~mm}$, at the throat $5-6 \mathrm{~mm}$ wide, slightly contracted above the spur and in the throat, hardly ventricose, villose outside, inside pubescent with glandular hairs in the throat; tube 38-42 $\mathrm{mm}$ long; spur obtuse, $2 \mathrm{~mm}$ long and wide; limb 17-22 mm wide; lobes subequal, obscurely repand-serrate, $6-10 \mathrm{~mm}$ in diam. Stamens included; filaments contorted, glabrous, all connate at the base, inserted on the base of the corolla; anthers orbicular, all coherent; cells divergent at the base, discrete, dehiscent throughout by a longitudinal split. Staminode very small, triangular, glabrous, about $0.5 \mathrm{~mm}$ long and wide, without anther. Ovary hirsute, ovoid, $4 \times 2.5 \mathrm{~mm}$; style about as long as the corolla tube, glabrous, slightly thickened towards the apex; stigma bilobed. Disk gland tongue-shaped, about $1.5 \mathrm{~mm}$ high. Placentae ovuliferous on the inner surface only. Seeds and fruit not seen.

Distribution: British Guiana.

Ecology: In rainforests.

British Guiana: Pakaraima Mts., Wenamu R., alt. 180 m., Davenport 7 (K, holotype); Schomburgk 37 (K).

10. E. reptans Mart., Nov. Gen. 3: 41, t. 217. 1829; G. Don, Gen. Syst. 4: 655, f. 70. 1838; D.G., Prod. 7: 546. 1839; Hanstein, Linnaea 26: 178, 207, 215, t. 2. f. 38. 1853; in Martius, Fl. Bras. 8 (1): 402. 1864; Linnaea 34: 342. 1865; Leeuwenberg, Baileya 5: 23. 1957; H. E. Moore, African Violets, Gloxinias, and their relatives 190, 276, 301 , f. $25 \mathrm{~A}$, f. $26 \mathrm{C}$. 1957.

Lectotype: BRAzIL: Amazonas, near R. Japurá, near Manacurú, Martius 3091 (M, photographs: U, US).

Heterotypic synonym: Cyrtodeira fulgida Linden, Cat. 90: 5. 1873; André, Illustr. Hortic. 20: 107, pl. 131. 1873. Type: Illustr. Hortic., icon. cit., depicted by Linden; no specimen preserved.

Homotypic synonym: Episcia fulgida (Linden) Hook. f., Bot. Mag. 100: t. 6136. 1874; H. E. Moore, Baileya 1: 46, f. 36B. 1953; op cit. 2: 75.1954.

Creeping stoloniferous herb. Leaves of very variable size, those of a pair equal or subequal; blade dull dark green above and with a creamy area along the midrib and often also along the veins, purple beneath, bullate, elliptic, acute or obtuse at the apex, rounded or subcordate at the base, hirsute on both sides. Flowers solitary or three together. Sepals green, lanceolate, acute at the apex, hirsute on both sides. Corolla scarlet, pinkish in the throat, trumpet-shaped, 3.5-4 cm long; tube nearly cylindric.

Terrestrial or epiphytic, about 5-20 cm high, up to $50 \mathrm{~cm}$ long. Stems terete, with spreading adventitious roots, hirsute. Stolons hirsute, $6-20 \mathrm{~cm}$ long. Leaves: petiole hirsute, $0.5-7 \mathrm{~cm}$ long; blade $1 \frac{1}{2}-2 \times$ as long as wide, $2-13 \times 1.3-9$ (in cult. plants up to $15 \times 10) \mathrm{cm}$, in young plants on stolons often smaller, membranaceous or papyraceous when dry, crenate-serrate; midrib and veins impressed 
above, prominent beneath. Inflorescence: peduncle obsolete or very short, hirsute, up to $5 \mathrm{~mm}$ long. Two bracts present when peduncle not obsolete, green, lanceolate, 4-5 $\times$ as long as wide, 5-12 $\times 1-3 \mathrm{~mm}$, acuminate, entire, hirsute on both sides. Pedicels slender, hirsute, $1-4 \mathrm{~cm}$ long. Sepals subequal, somewhat connate at the base, about $4 \times$ as long as wide, 7-15 (mostly 9-13) $\times 2-4 \mathrm{~mm}$, slightly narrowed towards the base, serrate near the apex, four erect, the fifth (dorsal) curved around the spur. Corolla oblique in the calyx, $2 \frac{1}{2}-4 \times$ as long as the calyx, at the base 3-4 $\mathrm{mm}$, at the throat 5-8 mm wide, shortly spurred at the base, hardly contracted above the spur, not so in the throat, slightly widened, not ventricose; hirsute outside, inside with a ring of glandular hairs in the throat; tube $3-3.5 \mathrm{~cm}$ long, slightly and gradually curved; spur obtuse, about as long as wide; limb $1.5-2.5 \mathrm{~cm}$ wide; lobes subequal, serrulate, 5-10 mm in diam. Stamens included; filaments glabrous, sometimes contorted after the pollen is shed, free from each other, inserted on the base of the corolla; anthers nearly rectangular, about $1.5 \times$ as long as wide, all coherent; cells parallel, discrete, dehiscent throughout by a longitudinal split. Staminode very small, tongue-shaped, glabrous, about $0.3 \mathrm{~mm}$ long, with an obscure sterile anther (in specimen cult. at Baarn, Netherlands, $2 \mathrm{~mm}$ long, with a conspicuous sterile anther). Ovary hirsute, ovoid, 4-5 $\times 2.5-3 \mathrm{~mm}$; style somewhat longer than the stamens, glabrous; stigma capitate. Disk gland obscurely bilobed, glabrous. Placentae ovuliferous on both surfaces. Capsule globose, hirsute, $10 \mathrm{~mm}$ in diam. Seeds dark brown, $2 \times$ as long as wide, $0.8 \times$ $0.4 \mathrm{~mm}$.

Distribution: Colombia, British and French Guiana, and Brazil (Amazonas, Rio Branco, and Minas Gerais).

Ecology: On rocks or on tree trunks, among mosses, in rainforests, near waterfalls, at low elevations.

British Guiana: Upper Mazaruni R., de la Cruz 2350 (NY, F, MO, PH, UC, US); Upper Rupununi R., near Dadanawa, de la Cruz 1787 (NY, F, MO, PH, UC, US); between Demerara and Berbice Rs., de la Cruz 1588 (NY, F, K, MO, PH, UC, US).

French Guiana: Leprieur, anno 1859 (G).

11. E. sphalera Lwbg., p. 310.

Fig. 27, p. 414

Type: Suriname: Kappler 2044 ( $P$, holotype, isotypes: GOET, $\mathrm{RO}, \mathrm{W}, 2$ sheets, distributed as Tussacia villosa).

Creeping stoloniferous herb. Leaves of very variable size, those of a pair equal or subequal; blade medium green above, paler beneath, bullate, elliptic or oblong-ovate, acute or obtuse at the apex, rounded or subcordate at the base, hirsute on both sides. Flowers solitary, often long-pedicellate. Sepals green, lanceolate, acute at the apex, hirsute on both sides. Corolla white (?) or mauve (?), obliquely infundibuliform, 24-25 mm long; tube suddenly curved in the middle and from that point more strongly widened, $15 \mathrm{~mm}$ long; limb $20-25 \mathrm{~mm}$ wide. Stamens slightly exserted when filaments not contorted.

Terrestrial. Stems with spreading adventitious roots, hirsute. Leaves: petiole hirsute, 5-40 mm long; blade 1.3-2 $\times$ as long as wide, $2-7 \times 1.5-4 \mathrm{~cm}$, membranaceous when dry, crenate-serrate. Inflorescence: pedicel slender, hirsute, $2-5 \mathrm{~cm}$ long. Sepals subequal, nearly free, $3-4 \times$ as long as wide, $6-9 \times 1.5-3 \mathrm{~mm}$, slightly narrowed towards the base, entire or with some teeth, four erect, the fifth (dorsal) somewhat smaller, curved around the spur. Corolla oblique in the calyx, at the base $3 \mathrm{~mm}$, at the throat $6-10 \cdot \mathrm{mm}$ wide, slightly contracted above the spur, not or hardly so in the throat, not ventricose, villose outside, inside with a ring of glandular hairs in the throat; spur obtuse, about $1.5 \times$ as long as wide; lobes 5 , subequal, crenate-serrate, $\cdot 6-10 \mathrm{~mm}$ in diam. Filaments glabrous, contorted or not, all connate at the base, inserted on the base of the corolla; anthers oblongreniform, all coherent; cells divergent at the base, confluent at the apex, partially 
dehiscent at the base. Staminode small, glabrous, $0.5 \mathrm{~mm}$ long, with a very small sterile anther. Ovary hirsute, ovoid, $2.5 \times 1.5 \mathrm{~mm}$; style pubescent with glandular hairs; stigma saucer-shaped or obscurely bilobed. Disk gland entire, glabrous. Placentae ovuliferous on both surfaces. Capsule globose, hirsute, $5 \mathrm{~mm}$ in diam. Seeds dark brown, $2 \times$ as long as wide, $0.5 \times 0.25 \mathrm{~mm}$.

Distribution: Suriname, French Guiana.

Ecology: No data extant.

SURINAmE: Kappler 2044 (P, holotype, isotypes: GOET, RO, W).

French Guiana: Lower Mana R., vic. of Codebert, Wachenheim 209 (P).

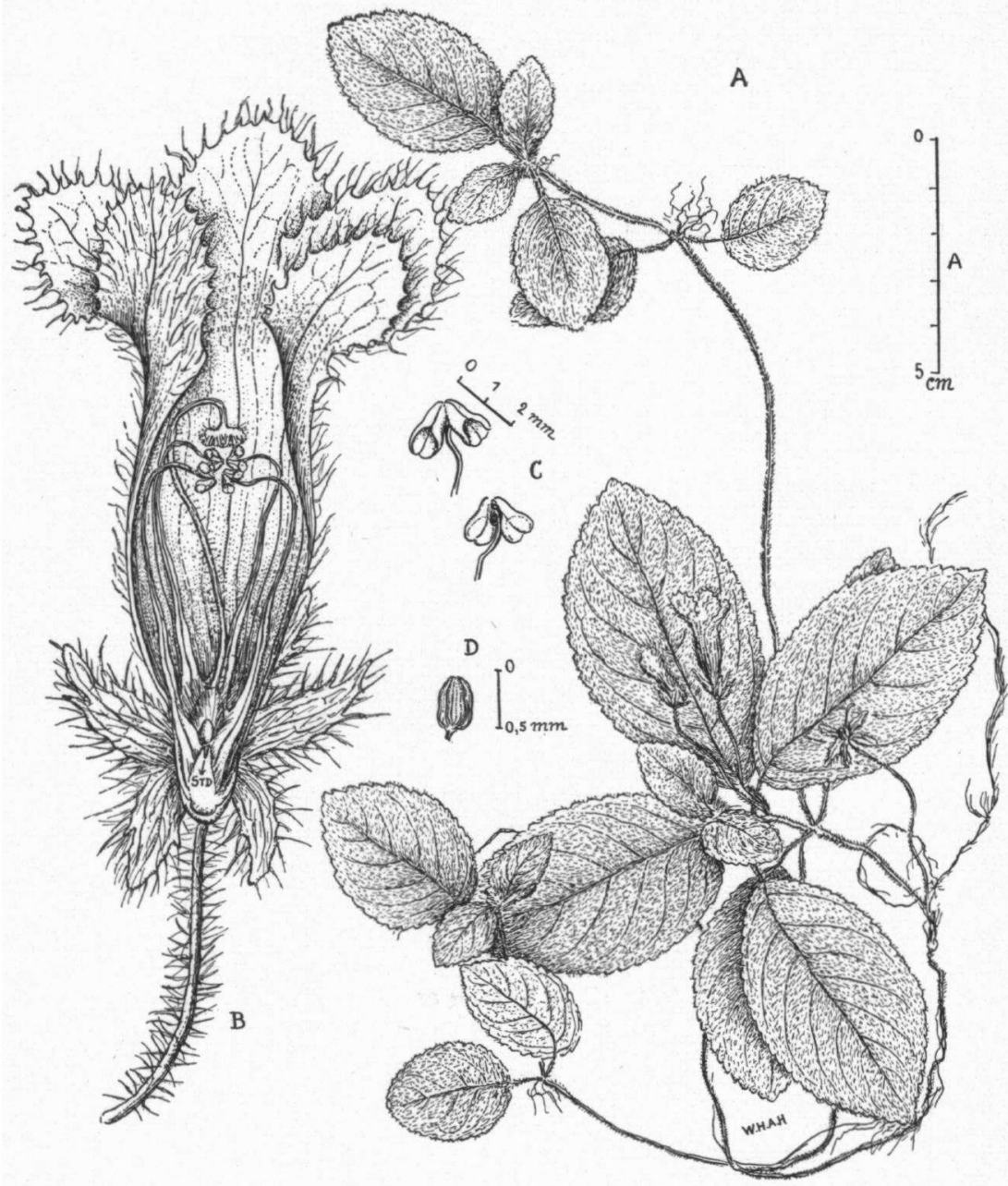

Fig. 27. Episcia sphalera: A. plant; B. opened flower; C. anther, both sides; D. seed (A and D: Kappler 2044, B-C: Wachenheim 209). Corolla often wider in the throat. 
8. Gloxinia l'Hérit., Stirp. Nov. 149. 1789 ("1784").

Terrestrial herbs, sometimes shrubby, $0.05-1.00 \mathrm{~m}$ high, with creeping rhizomes bearing scaly fleshy propagules or sometimes with stolons. Stems erect, mostly unbranched. Leaves opposite, those of a pair equal or subequal, petiolate, towards the apex often deminishing in size and changing into often sessile bracts; blade orbicular, ovate, or elliptic. Flowers axillary, solitary or sometimes 2 together, when leaves grading into bracts inflorescence terminal, racemose. Sepals green, leafy, nearly free, subequal, oblong. Corolla lilac, violet, pale blue, or pale purple, spotted or not, obliquely campanulate, ventrally often gibbous at the base, oblique in the calyx, not contracted in the throat, limb oblique, spreading; lobes 5 , subequal, nearly rounded, mostly broader than long. Stamens 4, didynamous, included; filaments contorted, inserted on the base of the corolla; anthers orbicular or nearly so, all coherent; cells parallel or divergent at the base, discrete, dehiscent throughout by a longitudinal split. Ovary inferior; hypanthium green, obovoid; style included; stigma stomatomorphic or bilobed. Disk annular, often narrow, entire or 5-lobed. Capsule obovoid, apically bivalved, included in the hypanthium. Seeds shining, ellipsoid, spirally striate.

Type species: G. perennis (L.) Fritsch (G. maculata l'Hérit.)

Distribution: About 6 species in tropical America.

1. G. perennis (L.) Fritsch in Engl. Prantl, Nat. Pflanzenf. 4 (3b): 174. 1894 ("1895"); in Engl., Bot. Jahrb. 50: 395. 1913; Notizbl. Berlin 6: 381. 1915; Pulle, Enum. 421. 1906; Boldingh, Fl. W.I. Eil. 365. 1913; Morton, Field Mus. Publ. Bot. 18: 1177. 1938; in Fl. Trin. and Tob. 2 (5): 314. 1955; Lemée, Fl. Guian. fr. 3: 464. 1953; H. E. Moore, African Violets, Gloxinias, and their relatives 141, 277, 301, f. 16, pl. IVB. 1957; Druce, Rep. Bot. Ech. Cl. Brit. Isles 3: 418. 1914 ("1913") (unnecessary new combination).

Basionym: Martynia perennis L., Sp. Pl. 618. 1753.

Type: Cult., Hort. Cliffortianus (BM). (Hort. Cliff. t. 18).

Homotypic synonyms: Gloxinia maculata l'Hérit., Stirp. Nov. 149. 1789 ("1784"); Sims, Bot.-Mag. 28: t. 1191. 1808; Reichenbach, Mag. aest. Bot. (= Icon. Pl. cult. et colend.) 1: t. 3. 1822; Savi, Fl. Ital. 3: 41, t. 98. 1824; G. Don, Gen. Syst. 4: 651. 1838; D.C., Prod. 7: 534. 1839; Hanstein, Linnaea 26: 169, 201. 1853; op. cit. 27: 716, 762. 1854; op. cit. 34: 432. 1865; in Martius, Fl. Bras. 8 (1): 419. 1864.

Salisia maculata (l'Hérit.) Regel, Bot. Zeit. 9: 894. 1851.

Gloxinia trichotoma Moench, Suppl. 194. 1802.

Salisia gloxiniaefolia Regel, Flora 32: 179. 1849.

Heterotypic synonyms: Gloxinia heterophylla Poepp. et Endl., Nov. Gen. 3: 9. 1845. Type: Peru: Loreto, near boundary of San Martín, Cochero, Poeppig 1619 (W, holotype, photographs: U, WAG).

Gloxinia trichantha Miq., Linnaea 22: 473. 1849. Type: Suriname: "Horto culta et arenosis spontanea", Focke 822 (U, 2 sheets). 
Stems green or often partially reddish, with spreading short red lines. Leaves deminishing in size towards the apex and changing into sessile or subsessile bracts; blade dark green above, paler green or sometimes only partially dark red or purple beneath, orbicular, ovate, or elliptic, often obliquely cordate, subcordate, or rounded at the base, sparingly strigillose above. Inflorescence terminal. Corolla lilac, violet, pale purple, or pale blue, with a purple or crimson blotch inside at the base, villose outside.

Plant with creeping rhizomes. Stems glabrous or with scattered hairs. Leaves: petiole glabrous or with scattered hairs, mostly about $2-6 \mathrm{~cm}$, up to $12 \mathrm{~cm}$ long; the bases of the petioles of a pair clasping the nodes of the stems; blade $1-1 \frac{1}{2} \times$ as long as wide, mostly about 5-14 $\times 3.5-10 \mathrm{~cm}$, up to $18 \times 14 \mathrm{~cm}$, membranaceous or papyraceous when dry, acute or sometimes obtuse or slightly acuminate at the apex, crenate-serrate, beneath glabrous or with scattered hairs on the midrib and veins. Flowers solitary, the lower ones in the axils of common leaves. Bracts orbicular or oblong-ovate, $1-1 \frac{1}{2} \times$ as long as wide, coloured and hairy as the leaves, deminishing in size to $10 \times 8 \mathrm{~mm}$, acute at the apex, rounded or cuneate at the base. Pedicels green, often with short red lines, slightly thickened towards the hypanthium, glabrous or with scattered hairs, $7-40 \mathrm{~mm}$ long. Sepals dark green inside, paler outside, spreading, 2-3 $\times$ as long as wide, $8-19 \times 3.5-8 \mathrm{~mm}$, acute or obtuse at the apex, narrowed towards the base, serrulate near the apex, glabrous or with scattered hairs on both sides. Corolla 25-40 mm long, at the throat 20-32 $\mathrm{mm}$ wide, ventrally gibbous at the base, inside at the base sparsely pubescent with glandular hairs; lobes entire, $10-12 \times 10-18 \mathrm{~mm}$. Filaments sparsely pubescent with glandular hairs, contorted, free from each other; anthers nearly triangular, all coherent in a circle; cells divergent at the base. Staminode oblong-triangular, sparsely pubescent with glandular hairs, $1.5-2 \mathrm{~mm}$ long, with a sterile anther. Ovary apically pubescent; hypanthium often with short red lines, 6-7 $\times 4 \mathrm{~mm}$; style sparsely pubescent with glandular hairs, $12-15 \mathrm{~mm}$ long; stigma stomatomorphic. Disk obscure, annular. Placentae ovuliferous on both surfaces. Capsule about $2 \frac{1}{2} \times$ as long as wide, apically pubescent, 10-18 $\times 4-7 \mathrm{~mm}$; hypanthium when in fruit with 10 longitudinal ribs. Seeds brown, about $2 \times$ as long as wide, $0.3 \times 0.15 \mathrm{~mm}$.

Distribution: Colombia, western Venezuela, Ecuador, Peru, Brazil (Amazonas), and possibly Guiana.

Ecology: In rainforests, sometimes on rocks, at middle and high elevations.

This species is perhaps not indigenous in Guiana. From this region it is only known by old collections some of which from gardens.

Suriname: Paramaribo, cult. and wild, Focke 822 (U, type of G. trichantha);: Splitgerber 102 (L); Paramaribo, in hort., Kegel 1430 (GOET); ibid., cult.,. Wullschlägel 767 (BR, GOET, U, W).

French Guiana: Leprieur, anno 1850 (P); L. G. Richard s.n. (P); in hort.,. Poiteau s.n. (LE).

Doubtful species:

2. G. suaveolens Dcne., Rev. Hortic. Sér. 3.2: 463.1848.

Homotypic synonym: Salisia suaveolens (Dcne.) Regel, Bot. Zeit. 8.894.1851.

Description highly insufficient. No specimens seen.

9. Kohleria Regel, Flora 31: 250. 1848.

Terrestrial herbs, subshrubs, or shrubs, $0.20-1.50 \mathrm{~m}$ high, often with rhizomes bearing scaly fleshy propagules or sometimes . with stolons. Stems erect or ascending at the base, branched or not, conspicuously hairy, glabrescent near the base. Leaves opposite or in. 
whorls of three, those of a pair or a whorl equal or subequal, petiolate; blade variously shaped, ovate to lanceolate, mostly conspicuously hairy. Inflorescence axillary or terminal, when terminal thyrsoid, many-flowered, with leafy bracts, when axillary flowers solitary or several in sessile or pedunculate umbels or cymes, with or without bracts. Sepals green or coloured, free, equal. Corolla variously coloured, tubular, erect or oblique in the calyx, 3-5 $\times$ as long as the calyx, not spurred, slightly widened at the very base, contracted just above it and in the throat, usually ventricose in the middle, conspicuously hairy outside; tube nearly cylindric; limb spreading; lobes 5, subequal, rounded or broadly rounded. Stamens 4, didynamous, often slightly exserted when filaments not contorted; filaments contorted or not, inserted on the base of the corolla; anthers orbicular or oblong, all coherent or free; cells parallel, discrete, dehiscent throughout by a longitudinal split. Ovary half to over two-thirds inferior; style thick, about as long as the stamens; stigma bilobed or stomatomorphic. Disk glands five, sometimes two dorsal ones connate or all of them united into an entire very thin annulus. Placentae ovuliferous on both surfaces. Capsule ovoid, the superior part bivalved, acuminate with an often slightly curved apex; valves sublignose. Seeds shining, obliquely ellipsoid, obliquely striate or reticulate-striate, or with a network of impressed lines.

Type species: $K$. hirsuta (H.B.K.) Regel.

Distribution: about 45 species in tropical America.

K. hirsuta (H.B.K.) Regel, Flora 31: 250. 1848; Hanstein, Linnaea 34: 441. 1865; Urban, Symb. Ant. 2: 369. 1901 ; H. E. Moore, Baileya 1: 99, f. 50A. 1953; Morton in Fl. Trin. and Tob. 2 (5): 313.1955.

Basionym: Gesneria hirsuta H.B.K., Nov. Gen. 2: 317, t. 189. 1818 ("1817"); D.C., Prod. 7: 530. 1839.

Type: Venezuela: Sucre, Cumana, Caripe, Humboldt \& Bonpland 330 (B, herb. Willdenow 11717, holotype, not seen, photograph in $U$; isotypes: $P, 2$ sheets!).

Homotypic synonyms: Isoloma hirsutum (H.B.K.) Regel, Bot. Zeit. 9: 893. 1851; Grisebach, Fl. Br. W.I. Isl. 459. 1861 ("1864").

Brachyloma hirsutum (H.B.K.) Hanst., Linnaea 26: 203. 1853; op. cit. 29: 526, 569. 1858.

Heterotypic synonym: Brachyloma rhodomallon Hanst., Linnaea 29: 526, 570. 1858. Type: Venezuela: Mérida, Moritz 414 (BR, CGE, $\mathrm{L}, \mathrm{P}$, isotypes).

Homotypic synonym: Kohleria rhodomallos (Hanst.) Hanst., Linnaea 34: 441. 1865; Fritsch in Engl., Bot. Jahrb. 37: 420. 1913.

Leafblade medium green above, paler green and often red-veined and red at the margin or rarely entirely red beneath, obliquely oblongovate or oblong-elliptic, obliquely cuneate or less often rounded at the base, sparsely tomentose above, often whitish-sericeous-tomentose beneath. Inflorescence axillary, 1-6-flowered. Peduncle obsolete or very short, up to $9 \mathrm{~mm}$ long. Pedicels elongate. Sepals $3.5-6 \times$ as long as wide. Corolla orange-red outside, inside orange-yellow, red- 
spotted on the limb and in the throat. Stamens slightly exserted when filaments not contorted.

Perennial herb, $0.20-1.20 \mathrm{~m}$ high, with rhizomes. Stems green, often reddish, often woody at the base. Leaves: petiole lanate-hirsute, 12-50 mm long; blade $2-3 \times$ as long as wide, $3-17 \times 1.5-8.5 \mathrm{~cm}$, papyraceous when dry, acuminate at the apex, serrate. Inflorescence: bracts, if any, very small, about $5 \mathrm{~mm}$ long, lanatehirsute. Pedicels often reddish, slender, lanate-hirsute, 25 $65 \mathrm{~mm}$ long, not thickened at the apex. Sepals green, often partially reddish, triangular to subulate, 6-10 $\times$ 1-2.5 mm, acuminate at the apex, entire, tomentose on both sides. Hypanthium tomentose, broadly obovoid, about as long as wide, 4-7 mm long. Corolla nearly cylindric, erect in the calyx, $2 \frac{1}{2}-4 \frac{1}{2} \times$ as long as the calyx, 23-35 mm long, at the base $3-4 \mathrm{~mm}$, at the throat $5-8 \mathrm{~mm}$ wide, densely red-villose outside except the glabrous base, glabrous inside, only on the limb densely pilose with capitate hairs; tube 19-32 $\mathrm{mm}$ long; limb $10-12 \mathrm{~mm}$ wide; lobes rounded, entire, $3-5 \mathrm{~mm}$ in diam. Filaments glabrous, free from each other; anthers oblong. Staminode small, glabrous, $2.5 \mathrm{~mm}$ long, with a small sterile anther. Ovary half to two-thirds inferior, superior part softly hirsute, $2.5 \times 2 \mathrm{~mm}$; style pubescent with capitate hairs; stigma white, bilobed. Disk glands five, equal, truncate, glabrous. Capsule $10-12 \times 7-8 \mathrm{~mm}$, superior part hirsute. Seeds dark brown, $1 \frac{1}{2}-3 \times$ as long as wide, $0.4-0.6 \times 0.15-0.25 \mathrm{~mm}$, with a network of impressed lines.

Distribution: Colombia, Venezuela, Trinidad, and British Guiana.

Ecology: Often on rocks, on mountains; alt. approximately 900$2000 \mathrm{~m}$.

BRITISH Guiana: Kanuku Mts., Iramaipang, F.D.-W.B. 623 (K).

\section{Lembocarpus Lwbg., p. 318.}

Small acaulescent terrestrial or sometimes epiphytic herbs, 5-20 cm high, with a small tuber bearing the leaves and the peduncles. Leaves usually solitary, petiolate; blade cordate-orbicular, acute or obtuse at the apex, cordate at the base, irregularly sinuate-dentate, subpalmate-veined. Inflorescence axillary, solitary, subcymose, few-flowered. Peduncle slender, about as long as the leaves, with some small bracts. Sepals 5, green, free, equal, linear-lanceolate, with three longitudinal veins. Corolla pale blue or tube white and limb lavender or white with purple dots inside on the limb, amply tubular or campanulate, oblique in the calyx, $2-4 \times$ as long as the calyx, not spurred, dorsally slightly gibbous at the base, not contracted above the gibbosity, nor in the throat, hardly ventricose; limb spreading, slightly oblique; lobes 5 , subequal, broadly rounded. Stamens 4 , didynamous, included; filaments not contorted, free from each other, inserted on the base of the corolla; anthers oblong, coherent in pairs; cells parallel, discrete, dehiscent throughout by a longitudinal split. Ovary superior; style included, deciduous; stigma bilobed. Disk annular, narrow, entire. Placentae ovuliferous only on the outer surface. Fruit a capsule, bivalved. Seeds (immature) shining, obliquely ellipsoid, longitudinally or obliquely striate.

Distribution: One species in the mountains of Suriname.

L. amoenus Lwbg., p. 319.

Fig. 28, p. 419

Type: Suriname: Nassau Mts., Maguire 40806 (U).

Leaves sometimes 2 together, petiole slender, mostly shorter than 
the blade. Corolla pale blue or tube white and limb lavender (changing during anthesis?).

Tuber globose with spreading adventitious roots, $2-15 \mathrm{~mm}$ in diam. Leaves: petiole pilose or villose, $0.5-10 \mathrm{~cm}$ long; blade medium green above, paler green or purplish beneath, slightly oblique, about as long as wide, $10-140 \times 7-140 \mathrm{~mm}$ (mostly about 50-80 $\times 45-75 \mathrm{~mm}$ ), thinly membranaceous when dry, often very sparingly strigillose above, sparsely strigillose beneath, especially on the midrib and veins; 3-7 longitudinal veins together at the base. Inflorescence 1-7-flowered.

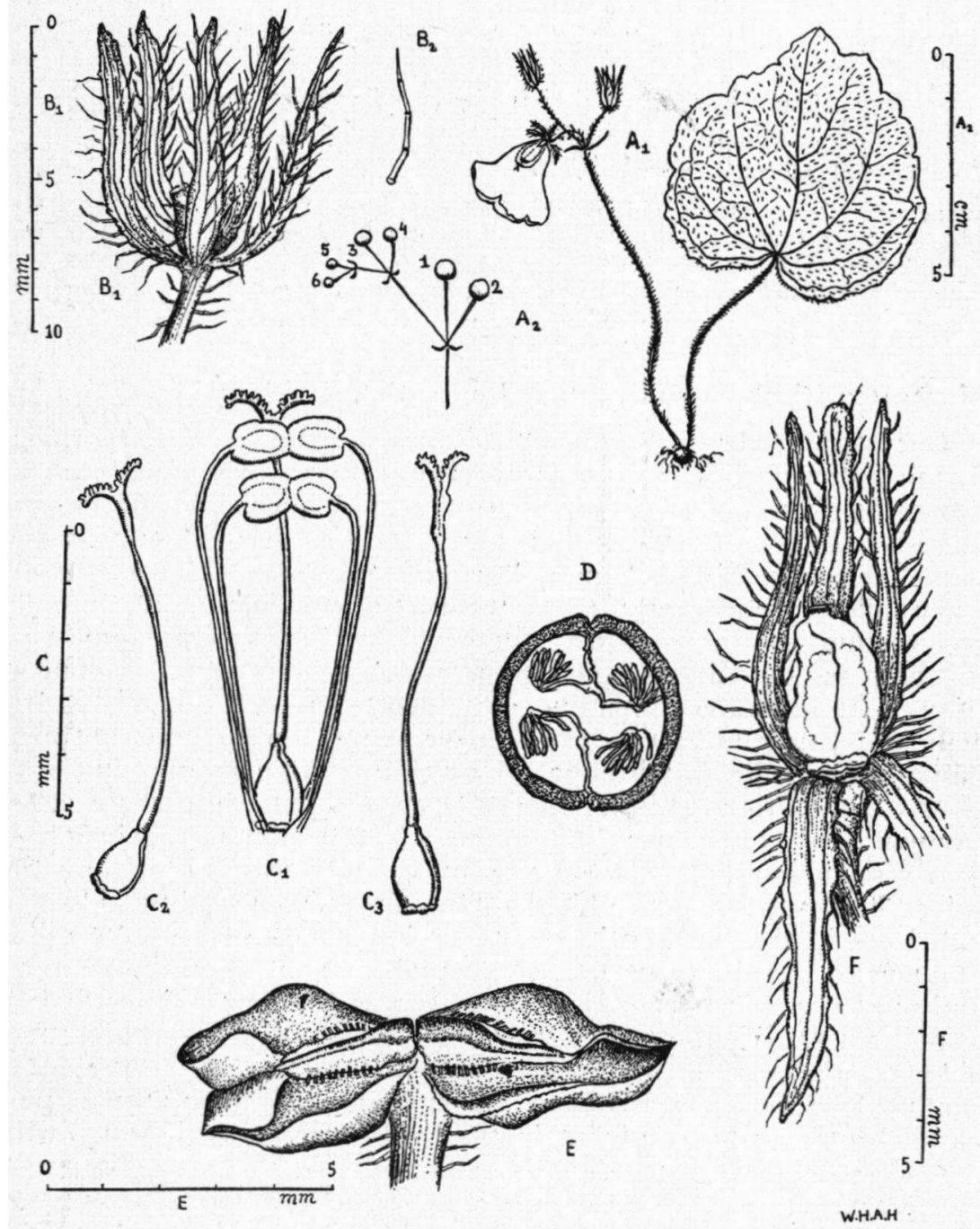

Fig. 28. Lembocarpus amoenus: $A_{1}$. plant; $A_{2}$. diagram of inflorescence; $B_{1}$. calyx with ovary; $B_{2}$. hair of calyx; $C_{1}$. pistil and stamens; $C_{2}$ and $C_{3}$. pistils; $D$. transverse section of ovary; $E$. open capsule; $F$. immature fruit (A-D and F: Lanjouw \& Lindeman 2833; E, B. W. 7077). 
Peduncle pilose or villose, 4-16 cm long. Bracts linear-lanceolate, $5-8 \times$ as long as wide, $2-10 \times 0.3-1.5 \mathrm{~mm}$, acute or acuminate, entire or obscurely dentate, pilose or villose on both sides. Pedicels pilose or villose, 5-20 mm long. Sepals $3 \frac{1}{2}-10 \times$ as long as wide, $5-11 \times 0.5-3 \mathrm{~mm}$, acuminate at the apex, entire or obscurely sinuate-dentate, villose with ordinary and capitate hairs outside, minutely puberulous or glabrous inside. Corolla $16-25 \mathrm{~mm}$ long, at the base $2-4 \mathrm{~mm}$, at the throat 9-16 $\mathrm{mm}$ wide, pilose or villose outside, glabrous inside; tube $12-20 \mathrm{~mm}$ long; limb 11-21 mm wide; lobes entire, ciliolate, 5-12 $\times 2-6 \mathrm{~mm}$. Filaments glabrous, gradually widened towards the base. Staminode very small, glabrous, $1.5 \mathrm{~mm}$ long, obtuse, without anther. Ovary puberulous, ovoid, $2-2.5 \times 1.5-2 \mathrm{~mm}$; style about as long as the stamens, glabrous. Disk glabrous. Capsule broadly ovoid, puberulous, 5-6 5 4-5 mm; valves chartaceous, glabrous and shining inside. Immature seeds medium brown.

Ecology: On moss-covered dripping rocks (once found on a small tree testibus Lanjouw \& Lindeman), on ferrite boulders in montane savanna-forests; alt. , 400-1 $100 \mathrm{~m}$.

Suriname: Nassau Mts., Lanjouw \& Lindeman 2466 (U), 2593 (U), 2833 (U); ibid., Maguire 40788 (U), 40806 (U, holotype); Wilhelmina Mts., B.W. 7077 (U); Emmaketen, B.W. 5807 (U).

Some additional specimens belong to Lembocarpus and perhaps to $L$. amoenus (see p. 319).

11. Napeanthus Gardn., Lond. Journ. Bot. 2: 13. 1843.

Terrestrial herbs, $5-45^{\circ} \mathrm{cm}$ high. Stems short or very short, rhizomatous, often woody. Leaves crowded at the apex, rosulate, sessile or subsessile, lanceolate, oblong-spathulate, oblanceolate, or linearlanceolate, very variable. Inflorescence axillary, usually subcymose, umbellate, or nearly so, 1-many-flowered. Peduncle with some bracts when inflorescence several-flowered. Bracts green, leafy. Calyx usually green, campanulate; sepals 5 , equal, valvate in the bud, connate at the base up to half of their length, oblong, lanceolate, or oblongtriangular, in flower spreading and inconspicuously veined, entire, acuminate, in fruit erect, enlarged, becoming thicker and conspicuously veined with longitudinal veins connected here and there by cross-veins. Corolla white or lilac with or without white at the base, campanulate, cup-shaped, or rotate, soon deciduous, glabrous or nearly so, about $1-2 \times$ as long as the calyx; tube shorter than the calyx; limb subactinomorphic or actinomorphic, spreading; lobes 5 , imbricate in the bud, entire, more or less emarginate at the apex. Stamens included or exserted, five, equal, or four, often didynamous and mostly with a staminode; filaments glabrous, not contorted, free from each other, inserted on the base of the corolla; anthers free, oblong, orbicular, or reniform; 'cells often divergent at the base, confluent at the apex, dehiscent throughout by a longitudinal split, parallel and hardly confluent in the bud. Staminode glabrous, with a sterile anther. Ovary superior, glabrous, ovoid or globose; style mostly curved; stigma saucer-shaped or obscurely bilobed. Disk none. Placentae ovuliferous on both surfaces. Capsule included in the calyx, 2- or 4-valved, apiculate by the persisting style; valves somewhat translucent, deciduous. Seeds brown, shining, ellipsoid, obliquely or sometimes longitudinally striate or reticulate-striate. 
Type species: $\mathcal{N}$. primulifolius (Raddi) Sandwith ( $\mathcal{N}$. brasiliensis Gardn.):

Distribution: 12 species in tropical America.

Key to the species:

Peduncle very slender; flowers small; flowering calyx $3 \mathrm{~mm}$ (or more ?), when fruiting up to $6 \mathrm{~mm}$ long; sepals connate at the base up to one-third of their length $\ldots \ldots \ldots 1$. N. jelskii Peduncle rather stout; flowers larger; flowering calyx about $7 \mathrm{~mm}$, when fruiting up to $13 \mathrm{~mm}$ long; sepals united for one-third to one-half of their length . . . . . . . . 2. N. sp.

1. N. jelskii Fritsch, Sitzungsb. Akad. Wiss. Wien 134, Abt. 1: 124. 1925.

Type: French Guiana: Cayenne, Jelski s.n., not seen, destroyed in Berlin.

Leaves lanceolate or oblong-spathulate, $2-4 \times$ as long as wide, $1.5-14 \times 0.8-4.5 \mathrm{~cm}$, membranaceous when dry, obtuse or rounded at the apex, remotely serrate-dentate, sinuate, or subentire. Peduncle filiform. Bracts small, 2-4 $\times 0.4-1 \mathrm{~mm}$. Sepals connate at the base to one-third of their length, lanceolate, about $3 \times$ as. long as wide, in flower $3 \times 1 \mathrm{~mm}$ (or more?), in fruit up to $6 \times 2 \mathrm{~mm}$. Corolla (only seen in bud) pilose outside; limb subactinomorphic. Stamens 4 with a staminode.

Plant 5-10 cm high. Stems very short, $0.5-2 \mathrm{~cm}$ long, glabrous. Leaves medium green above, paler beneath, sparsely and minutely pilose on both sides. Inflorescence cymose. Peduncle sparsely pilose, 2.5-6 cm long. Bracts lanceolate, about $4 \times$ as long as wide, acuminate, entire, pilose on both sides. Pedicels filiform, sparsely pilose, 10-25 mm long. Sepals pilose outside, glabrous inside. Corolla white (?), glabrous inside; lobes rounded. Filaments (in the bud) of the stamens and of the staminode equal, $0.5 \mathrm{~mm}$ long. Ovary globose, $1 \mathrm{~mm}$ in diam.; style glabrous; stigma obscurely saucer-shaped. Capsule one-third of the length of the calyx, globose, $1.5 \mathrm{~mm}$ in diam., bivalved. Seeds dark brown, about $2 \times$ as long as wide, about $0.3 \times 0.15 \mathrm{~mm}$.

Distribution: French Guiana.

Ecology: No data extant.

French Guiana: Acarouany R., Lower Mana R., Mélinon 58 (P); Leprieur, anno $1838(\mathrm{P})$.

The following specimens mav belong to a new snecies closelv related to $\mathcal{N}$. jelskii, but the material is insufficient for description. They differ by the leaves which are 4-12 $\times$ as long as wide, acute or acuminate, and more hairy above. Stamens 5 (or 4 with a staminode ?).

French Guiana: Cayenne, anonym. (G, U); De Candolle \& l'Héritier s.n. (G); Poiteau s.n. (K, P, U); L. C. Kichard s.n. (P).

Gutana: French?: Boordes (?) s.n. (C).

\section{N. sp.}

Several specimens have been collected in Guiana which resemble $\mathcal{N}$. primulifolius (Raddi) Sandwith (Webbia $22(1): 332.1956$ ) that is indigenous in southern Brazil (Rio de Janeiro, São Paulo). They differ by the following characters: Seeds reticulate-striate, not spinose. 
Leaves beneath with numerous stomata-groups, more than the specimens from southern Brazil. No complete corolla seen. In bud 4 stamens and one staminode.

Suriname: Emmaketen, Stahel 183 (U); Nassau Mts., Lanjouw \& Lindeman 2630 (U).

French Gulana: Leprieur, anno 1840 (P), s.n. (G); L. C. Richard s.n. (P); herb. expos. col. s.n. (P).

12. Nautilocalyx Linden, Cat. 1851: 12 (fide Hanstein, Linnaea 26: $181,207.1853$.

Terrestrial or sometimes epiphytic herbs, $0.20-1.20 \mathrm{~m}$ high. Stems sappy, erect, creeping, or ascending, branched or not, often near the base with spreading adventitious roots. Leaves opposite, those of a pair equal or subequal, petiolate or sometimes sessile; blade often purple beneath, oblong-elliptic to lanceolate, acute or acuminate at the apex. Flowers axillary, solitary, fasciculate, or in short-pedunculate umbels or cymes, with often sepal-like bracts. Sepals green or coloured, free or connate at the base, ovate to linear-lanceolate. Corolla white, creamy, or pale yellow, often purple-spotted or-lined, tubular, usually narrowly infundibuliform, $1 \frac{1}{2}-3 \frac{1}{2} \times$ as long as the calyx, spurred or gibbous at the base, hardly or not ventricose; limb spreading; lobes 5 , subequal, rounded. Stamens 4 , didynamous, included; filaments contorted, inserted on the corolla on or near the base; anthers orbicular or oblong, all coherent, coherent in pairs, or free; cells parallel, discrete, dehiscent throughout by a longitudinal split. Ovary superior; style included; stigma bilobed, capitate, or nearly so. Mostly one dorsal disk gland, rarely also a ventral one. Placentae ovuliferous usually only on the inner or rarely on both surfaces. Capsule bivalved. Seeds shining, ellipsoid, obliquely striate.

Type species: $\mathcal{N}$. bracteatus (Linden) Linden ex Planch.

Distribution: 10 species in Colombia, Peru, Venezuela, Guiana, and Brazil (Amazonas).

Key to the species:

Stems short-villose or -pilose at the apex; leafblades sparsely strigose to glabrous above; corolla inside often dorsally purple-dotted and dorsally with glandular hairs, limb serrate . . . . N. pictus

Stems lanate-villose at the apex; leafblades villose to subtomentose above; corolla inside purple-lined, with glandular hairs in the throat, limb entire . . . . . . . . N. villosus

1. N. pictus (Hook.) Sprague, Kew Bull. 1912: 88.

Basionym: Centrosolenia picta Hook., Bot. Mag. 77: t. 4611. 1851. Type: Cult. in Kew Gardens from a collection of Spruce in Brazil (K, photographs: U, US).

Homotypic synonym: Episcia picta (Hook.) Hanst. in Martius, Fl. Bras. 8 (1): 403. 1864; Linnaea 34: 349. 1865.

Misapplied names: Collandra picta (Hook.) Lem., Jardin fleuriste 2: pl. 214. 1852, not Hanst. 
Columnea (?) picta (Hook.) Lem. ex Hanst. in Martius, Fl. Bras. 8 (1): 422. 1864, not Karsten.

Heterotypic synonym: Nautilocalyx lacteus Sandwith, Kew Bull. 1931: 489. Type: British Guiana: Essequibo R., Moraballi Ck., near Bartica, Sandwith 3 (K, photographs: U, US).

Stems purple-villose or -pilose at the apex. Leafblades dark green and sometimes paler along the midrib above, paler green, purpleveined, or entirely purple beneath, sparsely strigose to glabrous above, appressed-pubescent or pilosulous beneath, especially on the midrib and veins. Sepals densely villose on both sides, especially outside. Corolla white, creamy, or rarely yellow (teste L. C. Richard), inside dorsally pubescent with glandular hairs and often with some purple dots, lobes more or less serrate. Style pubescent.

Plant 20-30 cm high. Stems purple or green, creeping or ascending, glabrescent. Leaves: petiole purple or green, villose or pilose, $1-8$ (mostly 1.5-5) cm long; blade often bullate, oblong-elliptic or oblong-lanceolate, $2-3 \frac{1}{2} \times$ as long as wide, $5.5-22.5 \times 2.5-10 \mathrm{~cm}$, papyraceous when dry, acuminate at the apex, cuneate at the base, crenate-serrate. Flowers solitary or aggregated. Peduncle obsolete or very short, with some sepal-like lanceolate bracts. Pedicels short, purple-villose, 3-15 mm long. Sepals green or purple, connate at the extreme base, linear-lanceolate, $4 \frac{1}{2}-10 \times$ as long as wide, long-acuminate, with some teeth, four erect, 12-25 $\times$ $1.5-4 \mathrm{~mm}$, the fifth (dorsal) somewhat smaller, curved around the spur. Corolla $1 \frac{1}{2}-3 \frac{1}{2} \times$ as long as the calyx, 3-4.5 cm long, at the base $2-4 \mathrm{~mm}$, at the throat $7-11 \mathrm{~mm}$ wide, spurred at the base, slightly contracted above the spur and in the throat, more or less pilose outside; tube more or less infundibuliform, 25-35 mm long; spur obtuse, $1-1 \frac{1}{2} \times$ as long as wide, 1.5-5 $\times 1-4 \mathrm{~mm}$; limb 15-20 mm wide; lobes 4-7 mm in diam., two dorsal ones somewhat smaller than the others. Filaments glabrous or with some hairs near the apex, contorted, all connate at the base, inserted on the base of the corolla; anthers orbicular, coherent in pairs or all coherent, rarely free; connective mostly sparsely pilose. Staminode very small, glabrous, $0.3-1.3 \mathrm{~mm}$ long, apiculate, without anther. Ovary hirsute, ovoid, 2.5-4 × 1.5-3 mm; style filiform; stigma bilobed. Disk gland one, dorsal, large, nearly triangular, entire, glabrous; sometimes a tongue-shaped entire glabrous ventral gland. Placentae ovuliferous on the inner surface only. Capsule globose, about half as long as the calyx, pubescent, $6-8 \mathrm{~mm}$ in diam. Seeds dark brown, $1 \frac{1}{2}-2 \times$ as long as wide, $0.6-0.8 \times 0.3-0.5 \mathrm{~mm}$, obtuse at the apex, mucronate at the base.

Distribution: Guiana and northern Brazil.

Ecology: In rainforests; alt. 0-600 m.

British Guiana: Kurupung R., Jacoba, Lang \& Persaud 155 (F); Kurupung R., Kumaka, Altson 422 (K); Mazaruni R., Jenman 5457 (K); Upper Mazaruni R., de la Cruz 2327 (NY, F, MO, US); Kopinang R., Anandabaru, Altson 484 (K); Kaieteur Falls, Appun 1872 (BM); Potaro R. Gorge, Waratuk Falls, Maguire \& Fanshawe 23047 (NY, K, US); Kangaruma-Potaro Landing, Gleason 199 (NY, K, US); Cuyuni R., Appun 338 (K); ibid., Kartabo Point, Mell \& Mell 200 (US); Cuyuni R., north of Tinamu Fall, Martyn 303 (K); Essequibo R., Jenman 1297 (K); ibid., Persaud 350 (F); Mazaruni Station, Sandwith 1047 (K, G, S, U); ibid., Tutin 84 (BM); Bartica Grove, Jenman 2364 (K); Essequibo R., Moraballi Ck., near Bartica, Sandwith 3 (K, holotype of $\mathcal{N}$. lacteus) ; Essequibo R., Unabaruka Ck., Flemming's Camp, Martyn 226 (K); Pomeroon distr., Mt. Russell, Jenman 2080 (K); Rockstone, Gleason 611 (NY, K, US); Demerara R., Vrijheid, Altson 110 (K); between Demerara and Berbice Rs., de la Cruz 1584 (NY, F, MO, US). SuRINAmE: between Upper Gran Rio and Franssen Herderschee Piek, Tresling 411 (U); Nassau Mts., Lanjouw \& Lindeman 2686 (U), 2890 (U), 2941 (U); ibid., Maguire 40808 (NY).

French Guiana: Marowijne R., across from Nassau Mts., Cowan \& Lindeman 
39219 (NY); Aroura distr., Aublet s.n. (BM, identified as Episcia melittifolia); Leprieur, anno 1850 (P), s.n. (P, U); Martin 274 (BM), s.n. (BM); Martin, herb. Rudge s.n. (BM); Poiteau s.n. (K); L. C. Richard s.n. (P).

Guiana: British ?: Talbot, anno 1840 (K); French?: anonym. (Martin?) (BM); herb. J. E. Smith 1058. 11 (LINN).

2. N. villosus (Kunth et Bouché) Sprague, Kew Bull. 1912: 89 Basionym: Drymonia villosa Kunth et Bouché, Ind. Sem. Hort. Berol. 1847: 12 (fide Walpers, Ann. 1: 474. 1848-1849); Hooker, Bot. Mag. 81: t. 4866 . 1855.

Type not seen, destroyed in Berlin?

Homotypic synonym: Episcia villosa (Kunth et Bouché) Hanst., Linnaea 34: 348. 1865.

Stems lanate-villose at the apex. Leaf blades dark green above, paler green or purple beneath, more or less villose or subtomentose on both sides, beneath especially on the midrib and veins. Sepals villose outside, inside glabrous or only apically villose. Corolla white, inside with longitudinal purple lines and with glandular hairs in the throat; lobes entire. Style glabrous.

Plant about 20-30 cm high. Stems purple or green, erect or ascending, glabrescent. Leaves: petiole lanate-villose, $1-7 \mathrm{~cm}$ long; blade elliptic or oblong-elliptic, variable in size, $1 \frac{1}{2}-2 \times$ as long as wide, up to $16 \times 8.5 \mathrm{~cm}$, membranaceous or papyraceous when dry, acuminate at the apex, cuneate at the base, coarsely serrate or crenateserrate. Flowers 1 -several, mostly less than in $\mathcal{N}$. pictus. Peduncle obsolete or very short, with some sepal-like bracts. Pedicels villose, 5-20 mm long. Sepals green, connate at the base, linear-lanceolate, $6-10 \times$ as long as wide, long-acuminate, entire or with some teeth, four erect, 11-20 $\times 1.5-2.5 \mathrm{~mm}$, the fifth (dorsal) somewhat smaller, curved around the spur. Corolla $2-3 \frac{1}{2} \times$ as long as the calyx, $4-5.5 \mathrm{~cm}$ long, at the base $2-4 \mathrm{~mm}$, at the throat $7-15 \mathrm{~mm}$ wide, spurred at the base, slightly contracted above the spur and in the throat or not, pilose or villose outside; tube more or less infundibuliform, 3-4.5 cm long; spur obtuse; limb 2-3 $\mathrm{cm}$ wide; lobes $7-11 \mathrm{~mm}$ in diam. Filaments glabrous or with a single hair, contorted, all connate at the base, inserted on the base of the corolla; anthers orbicular or nearly so, coherent in pairs; connective glabrous. Staminode very small, glabrous, 0.3-0.5 mm long, acuminate, without anther. Ovary hirsute, ovoid, 4-5 $\times 2.5-3$ $\mathrm{mm}$; style filiform, stigma obscurely bilobed. Disk gland one, dorsal, large, entire, glabrous. Placentae ovuliferous on the inner surface only. Fruit and seeds not seen.

Distribution: Venezuela; Guiana?

Ecology: In shady places (teste Pittier).

French Gulana: St. Jean, Benoist 820 (P)? (see p. 320).

\section{Rechsteineria Regel, Flora 31: 247. 1848.}

Terrestrial herbs with tubers, up to $1.20 \mathrm{~m}$ high. Stems erect, branched or not. Leaves opposite, subopposite, or in whorls of 3-5, those of a pair or a whorl equal or subequal, petiolate or sessile; blade oblong, lanceolate, orbicular, or subcordate. Flowers solitary or fasciculate, axillary, in pedunculate axillary umbels or cymes, or in terminal thyrsoid or paniculate inflorescences. Sepals 5, green, reddish, or purplish, usually partially united. Corolla tubular, erect in the calyx, 3-5 $\times$ as long as the calyx, sub-globose at the base or especially dorsally gibbous, contracted just above the base and in the throat, 
more or less ventricose and curved; limb subactinomorphic or bilabiate (Guianan spp.), when bilabiate dorsal lip erect, and bilobed, and ventral lip spreading, half as long as the dorsal one, of three lobes. Stamens 4, didynamous, included or exserted; filaments not contorted, inserted on the base of the corolla; anthers all coherent in a square or in a circle; cells parallel, discrete, dehiscent throughout by a longitudinal split. The base of the ovary adnate to the base of the calyx; style filiform, slightly longer than the stamens; stigma stomatomorphic. Disk glands 1-5. Placentae ovuliferous on both surfaces. Capsule ovoid, acuminate with an often slightly curved apex, bivalved; valves sublignose. Seeds shining, fusiform, longitudinally reticulate-striate.

Type species: $R$. allagophylla (Mart.) Regel.

Distribution: About 75 species in Mexico and South America from Colombia to northern Argentina and Uruguay.

Key to the species:

Calyx lobes long-acuminate, $1-2 \times$ as long as the tube; leaves villose or pilose; flowers always axillary . . . 2. R. schomburgkiana

Calyx lobes acute or acuminate, $\frac{1}{2}-1 \times$ as long as the tube; leaves tomentose; inflorescence axillary or terminal . . 1 . R. incarnata

1. R. incarnata (Aubl.) Lwbg., p. 320. Figs. 29, p. 426; 30I, p. 428 Basionym: Besleria incarnata Aubl., Hist. Pl. Guian. 2: 635, and 4: t. 265. 1775; G. Don, Gen. Syst. 4: 652. 1838; D.C., Prod. 7: 539. 1839; Hanstein, Linnaea 34: 421. 1865.

Type: French Guiana: basin of Galibi Ck., Aublet s.n. (BM, flowers lost).

Heterotypic synonyms: Gesneria chelonioides H.B.K., Nov. Gen. 2: 315. 1818 ("1817"); G. Don, l.c., p. 647; D.C., l.c., p. 527; Hanstein, Linnaea 34: 262. 1865 (as Gesnera). Type: Colombia: Cauca, near the glens of the R. Guilguase and R. Smitha, between Popayan and Amalguer, Humboldt \& Bonpland s.n. (P-BO, photographs: F, U, US).

Homotypic synonyms: Corytholoma chelonioides (H.B.K.) Regel, Gartenflora 7: 374. 1858.

Rechsteineria chelonioides (H.B.K.) Kuntze, Rev. 2: 474. 1891.

Gesneria rutila Lindl., Bot. Reg. 14: t. 1158. 1828; Martius, Nov. Gen. 3: 34.1829 (as Gesnera); G. Don, l.c., p. 648; D.C., l.c., p. 529; Hanstein in Martius, Fl. Bras. 8(1): 372. 1864; Linnaea 34: 270.1865 (as Gesnera). Type: Cult. from South America, C. de Vandes, Sept. 1827 (GGE, holotype, photographs: U, WAG).

Homotypic synonyms: Corytholoma rutilum (Lindl.) Dcne.,. Rev. Hortic. Sér. 3. 2: 467.1848.

Rechsteineria rutila (Lindl.) Kuntze, 1.c.

Gesneria lindeniana Brongn., Rev. Hortic. Sér. 3. 1: 363. 1847. Type: Venezuela: Mérida, Linden 1408 (G, holotype, isotypes: BM, $\mathrm{F}, \mathrm{G}, \mathrm{K}, \mathrm{P}, \mathrm{W}, 2$ sheets).

Gesneria stachydifolia Benth., Pl. Hartweg. 230. 1846; Hanstein, Linnaea 34: 263. 1865 (as Gesnera stachyfolia). Type: Colombia: 
Cauca, vic. of Popayan, opposite the junction of $R$. Sucio with $R$. de las Botas, Hartweg 1259 (K, holotype, photographs: U, WAG). Homotypic synonym: Rechsteineria stachydifolia (Benth.) Kuntze, l.c.

Leafblades tomentose on both sides. Inflorescence terminal, thyrsoid. Bracts leafy, deminishing in size towards the apex or not. If the bracts do not deminish in size the flowers stand actually in the axils of ordinary leaves. Axillary part of inflorescence 1-5-flowered. Calyx campanulate, pubescent on both sides; lobes $\frac{1}{2}-1 \times$ as long as the tube, triangular, 1-2 $\times$ as long as wide, acute or acuminate, entire.

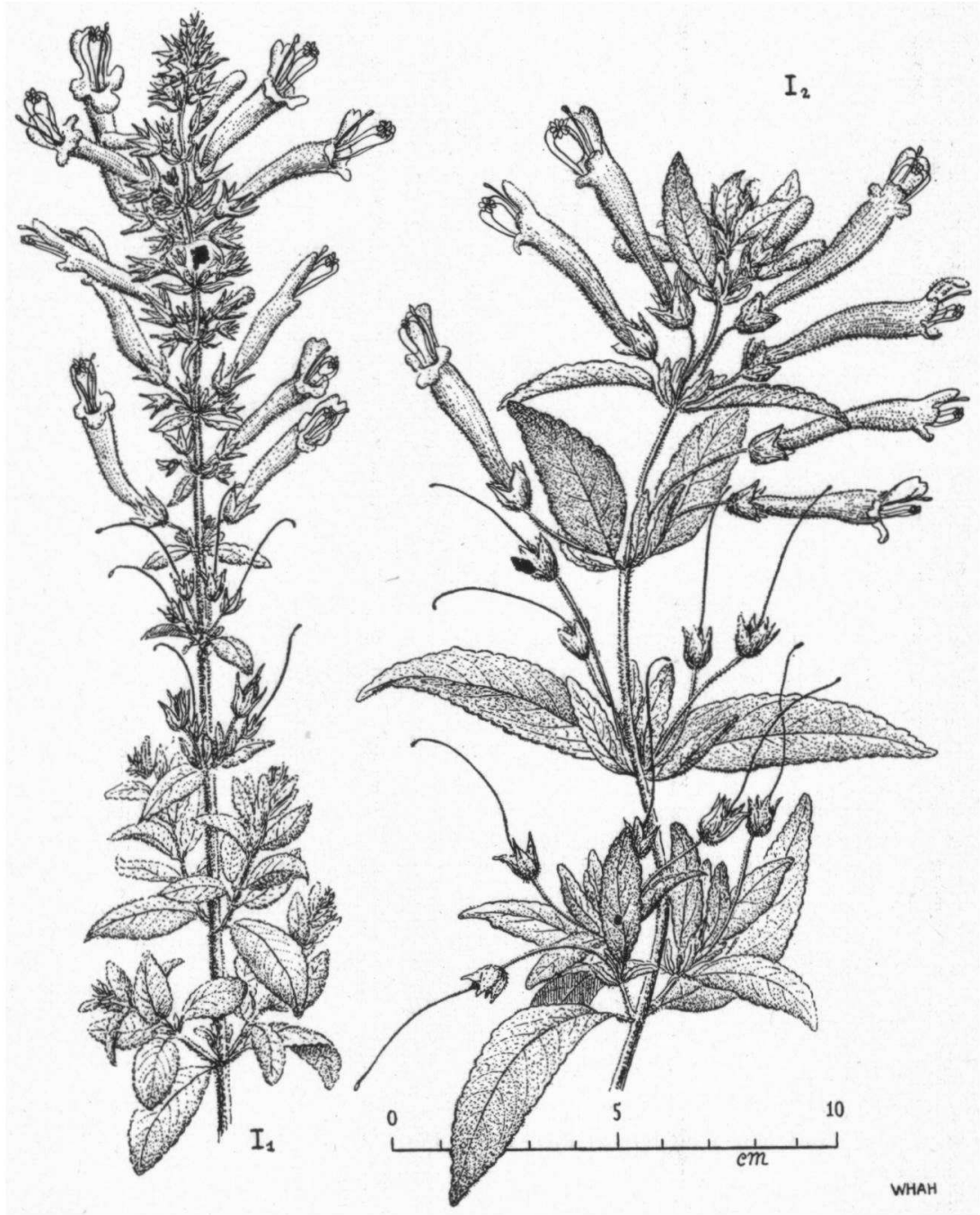

Fig. 29. Rechsteineria incarnata, branches, ( $I_{1}:$ Rombouts $505, I_{2}:$ Jenman 1908). 
Corolla red, yellow inside and outside at the base, sparsely pubescent outside; limb bilabiate.

Plant $0.15-1.20 \mathrm{~m}$ high. Tuber about $2.5-8 \mathrm{~cm}$ long. Stems often reddish, appressedpubescent to hirsutulous. Leaves opposite or rarely in whorls of three, petiolate or sessile; petiole appressed-pubescent or hirsutulous, 1-15 mm long; blade medium

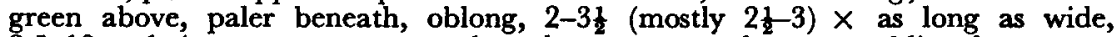
2.5-10 $\times 1-4 \mathrm{~cm}$, papyraceous when dry, acute at the apex, obliquely cuneate at the base, crenate. Inflorescence: axillary part: peduncle very short or obsolete, with two small bracts, these oblong-lanceolate, $2-3 \times$ as long as wide, 3-15 $\times 1-4$ $\mathrm{mm}$, acuminate, entire. Pedicels pubescent, $0.5-4 \mathrm{~cm}$ long. Calyx green or reddish, 7-14 $\times 6-9 \mathrm{~mm}$; lobes 4-7 $\times 3-6 \mathrm{~mm}$. Corolla erect in the calyx, 30-50 mm long, dorsally gibbous at the base, slightly contracted above the gibbosity and there 3-5 mm wide, somewhat ventricose, slightly contracted in the throat and there 5-8 $\mathrm{mm}$ wide, glabrous inside; tube 25-38 mm long; dorsal lip 5-8 $\times$ 4-7 mm, rounded, bilobed; ventral lip: lobes broadly rounded, $2-3 \times 4-5 \mathrm{~mm}$; all lobes entire. Stamens exserted; filaments glabrous, free from each other; anthers nearly rectangular. Staminode small, lanceolate, $1.5-3 \mathrm{~mm}$ long, long-acuminate, glabrous, curved into the gibbosity, without anther. Ovary almost entirely superior, pubescent, ovoid or conic, 3-5 $\times 2-3 \mathrm{~mm}$; style puberulous. Disk of one large dorsal mostly bilobed, sometimes entire or cleft gland, sometimes three additional glands, two lateral and one ventral, present. Capsule somewhat longer than the calyx, pubescent, 10-15 × 4-7 mm. Seeds dark brown, about 1-1.2 mm long.

Distribution: Colombia, Venezuela, Guiana, Brazil (Amazonas).

Ecology: On usually granitic rocks or on savannas, at low and middle elevations.

British Gutana: Pomeroon distr., Mt. Russell, Jenman 1908 (K); Upper Takutu R., Appun 2125 (K); Rupununi R., Appun 1581 (K); ibid., Sand Ck., F.D.-W.B. 70 (K); Demerara R., Parker s.n. (K).

Suriname: Corantijn R., above Frederik Hendrik Falls, Rombouts 158 (U); Upper Sipaliwini R., Rombouts 505 (U); ibid., Morro Grande, Rombouts 474 (U); Wilhelmina Mts., B.W. 7033 (U); vic. of Tanjimama R., Coppename R., Mennega 482 (U); Upper Saramacca, R. Pulle 492 (U).

French GuIANA: basin of Galibi Ck., Aublet s.n. (BM, holotype); bank of La Comté R., L. G. Richard s.n. (P); Cayenne, Martin s.n. (BR); ibid., Mélinon, anno $1846(\mathrm{P})$; Perrottet, anno $1819(\mathrm{P}, \mathrm{G})$, anno $1822(\mathrm{G})$; Poiteau s.n. (K).

2. R. schomburgkiana (Kunth et Bouché) Kuntze, Rev. 2: 474. 1891 (as $R$. schomburgkii). $\quad$ Fig. 30 S, p. 428

Basionym: Gesneria schomburgkiana Kunth et Bouché, Ind. Sem. Hort. Berol. 1844: 11 (fide Linnaea 18: 501. 1844); Hanstein, Linnaea 34: 270. 1865 (as Gesnera schomburgkii).

Type: BRITISH Guiana: Kanuku Mts., Schomburgk 118 (holotype destroyed in Berlin, isotypes: BM, $\mathrm{K}$ !; the $\mathrm{K}$ sheet is the holotype of Gesneria guianensis, photographs in U and US).

Homotypic synonym: Gesneria guianensis Benth., Lond. Journ. Bot. 5: 360.1846.

Leafblades villose to pilose on both sides, beneath especially on the midrib and veins. Flowers axillary, 1-2 together. Calyx campanulate, villose outside, pubescent inside; lobes $1-2 \times$ as long as the tube, triangular, long-acuminate, entire. Corolla red, villose outside with ordinary and glandular hairs; limb bilabiate.

Plant 0.15-1.00 m high. Tuber about $5 \mathrm{~cm}$ in diam. Stems villose. Leaves opposite or subopposite, petiolate, the basal ones sometimes sessile; petiole villose, $3-30 \mathrm{~mm}$ long; blade medium green above, paler beneath, oblong, about $2 \times$ as long as 
wide, 7-14 $\times 3.5-7 \mathrm{~cm}$, papyraceous when dry, acute at the apex, cuneate or rounded at the base, crenate. Inflorescence: peduncle very short or obsolete. Bracts none. Pedicels slender, villose, 8-32 mm long. Calyx green, 9-12 $\times$ 5-7 mm. Corolla 4-5 $\times$ as long as the calyx, 40-50 mm long, dorsally gibbous at the base, above the gibbosity $3-5 \mathrm{~mm}$ wide, dorsally somewhat ventricose, slightly contracted in the throat and there 5-8.5 mm wide, glabrous inside; tube 31-36 mm long; dorsal lip rounded, 5-6 mm in diam., bilobed; ventral lip: lobes $2 \times 4 \mathrm{~mm}$; all lobes entire. Stamens slightly exserted; filaments glabrous, free from each other; anthers nearly rectangular. Staminode small, oblong-triangular, $1.5 \mathrm{~mm}$ long,
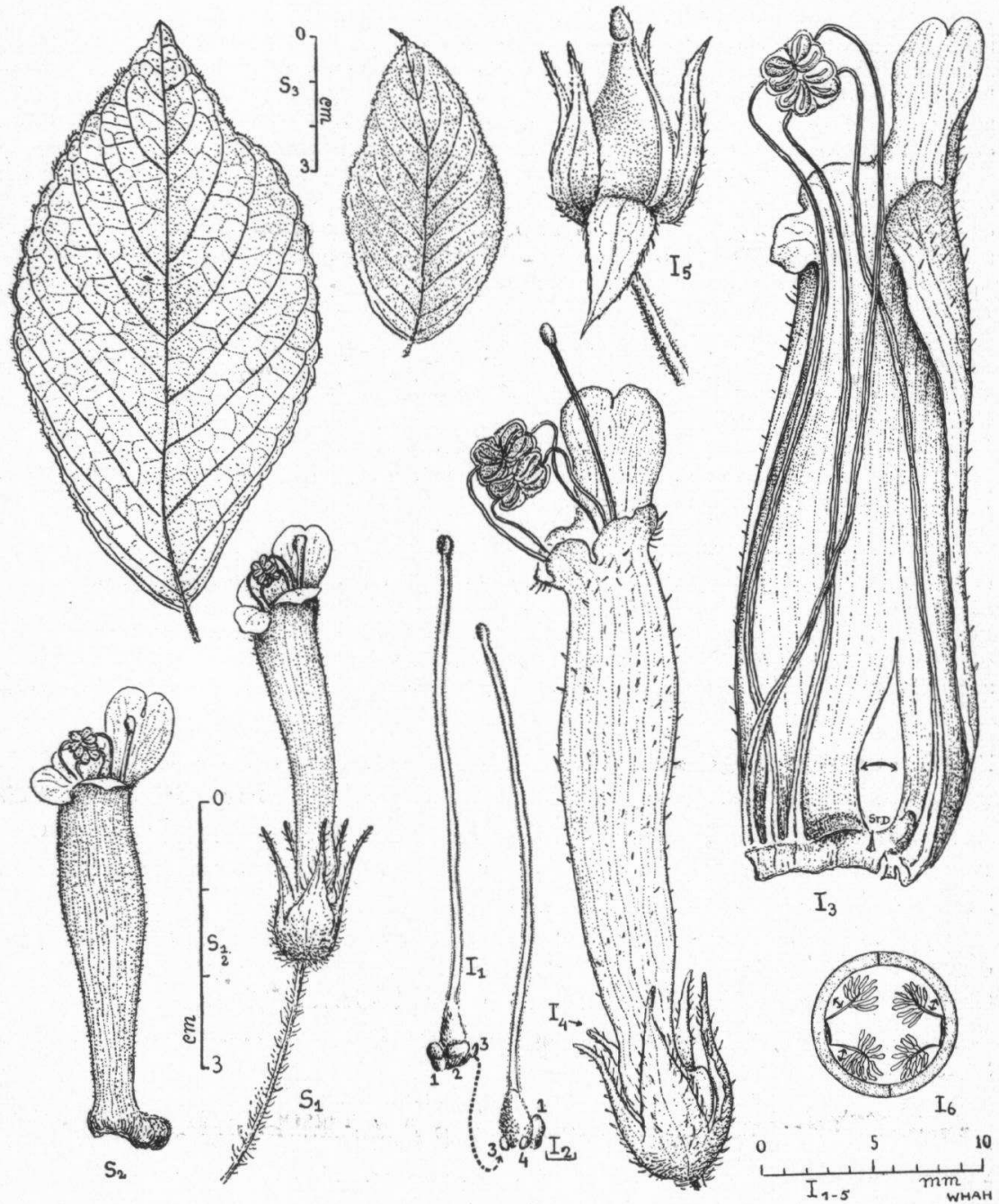

Fig. 30. Rechsteineria incarnata (Rombouts 505): 1. pistil dorsal; 2. pistil lateral; 3 . opened corolla; 4. flower; 5 . capsule; 6 . diagrammatic transverse section of ovary; S. $R$. schomburgkiana (A. C. Smith 3652 ): 1. flower; 2 . corolla with style; 3. leaves. 
acuminate, glabrous, curved into the gibbosity, without anther. Ovary one-third inferior, pubescent, ovoid, 4-5 × 3-4 $\mathrm{mm}$; style puberulous. Disk glands four, one large dorsal bilobed one and three small ones, one ventral and two lateral. Capsule about as long as the calyx, sparsely pubescent, $10-12 \times 5-7 \mathrm{~mm}$. Seeds dark brown, about $0.5 \mathrm{~mm}$ long.

Distribution: British and French (?) Guiana.

Ecology: On granitic rocks, on mountains.

British Guiana: Kanuku Mts., Schomburgk 118 (BM, K, isotypes); ibid., Mt. Iramaipang, alt. $975 \mathrm{~m}$, A. C. Smith 3652 (NY, F, K, U, US).

French Guiana: Poiteau s.n. (not seen, cited by Hanstein, 1865, p. 270, destroyed in Berlin ?).

14. Rhoogeton Lwbg., p. 321.

Terrestrial acaulescent herbs, 5-10 cm high, with (or without ?) small tubers bearing adventitious roots. Leaves radicular, petiolate; blade rounded, ovate, or oblong-ovate, often proliferous at the margin with small bulb-shaped (?) rooting plants, pinnately veined. Inflorescence axillary, umbellate, subcymose, or thyrsoid, long-pedunculate, 1-6-flowered, with some small lanceolate bracts. Sepals 5, green, free, oblong or lanceolate, acute, with 3 longitudinal veins. Corolla orange or red (?), nearly trumpet-shaped, oblique in the calyx, slightly widened, shortly spurred at the base, slightly contracted above the spur and in the throat, somewhat ventricose, slightly bent downwards, sparsely pubescent or strigillose outside, inside with glandular hairs in the throat; limb spreading, lobes 5, more or less unequal, rounded. Stamens 4, didynamous, included; filaments contorted or not, free from each other, inserted on the corolla near the base; anthers orbicular, square, or oblong, all coherent, coherent in pairs, or free; cells parallel, discrete, dehiscent throughout by a longitudinal split. Staminode very small, without anther. Ovary superior; style erect, included; stigma bilobed. Disk gland one, dorsal, large, glabrous, emarginate. Placentae ovuliferous on the outer surface only. Fruit and seeds not seen.

Type species: $R$. cyclophyllus Lwbg.

Distribution: 2 species in the mountains of British Guiana.

Key to the species:

Leafblades about as long as wide, rounded at the apex, cordate at the base, largely crenate-serrate . . . . 1. R. cyclophyllus Leafblades about $2 \times$ as long as wide, acuminate at the apex, rounded or obliquely cuneate at the base, biserrate-dentate 2 . R. viviparus

1. R. cyclophyllus Lwbg., p. 322.

Fig. 31, p. 430

Type: British Guiana: Mt. Ayanganna, Pakaraima Mts., Maguire, Bagshaw \& Maguire 40585 (NY, holotype, photographs: U, WAG).

Leafblades orbicular or nearly so, rounded at the apex, cordate at the base, crenate-serrate. Corolla orange, 20-22 mm long; lobes unequal.

Delicate small plant, $5-15 \mathrm{~cm}$ high. Tuber $2-4 \mathrm{~mm}$ in diam. Leaves: petiole slender, sparsely pubescent, 5-30 mm long; blade medium green above, paler 
beneath, 1-11 $\times$ as long as wide, 15-45 $\times 15-35 \mathrm{~mm}$ or smaller, membranaceous when dry, pubescent above, with scattered hairs beneath. Inflorescence subcymose or thyrsoid, 1-6-flowered. Peduncle $-4 \times$ as long as the leaves, sparsely pubescent, 4-15 cm long. Bracts opposite, oblong-lanceolate, $3-5 \times$ as long as wide, 3-8 $\times$ 0.5-2 mm, acute, entire, pubescent. Pedicels pubescent, 5-10 mm long. Sepals entire, sparsely pubescent on both sides, four subequal, oblong-lanceolate, $2-4 \times$ as long as wide, $2.5-4 \times 1-1.5 \mathrm{~mm}$, the fifth (dorsal) somewhat smaller and narrower, curved around the spur. Corolla $4-5 \times$ as long as the calyx, at the base $2 \mathrm{~mm}$, at the throat $5 \mathrm{~mm}$ wide, sparsely pubescent outside; tube 14-16 $\mathrm{mm}$ long; limb 11-17 mm wide; lobes obscurely crenate-serrate, one ventral large, obovate, 6-9 9 5-7 mm, two lateral ones rounded, 4-6 mm in diam., two dorsal ones rounded, 3.5-4 mm in diam. Filaments glabrous, not contorted; anthers orbicular or nearly so, all coherent or free. Staminode triangular, glabrous, $0.15 \mathrm{~mm}$ long. Ovary glabrous, ovoid, $2 \times 1.5 \mathrm{~mm}$; style glabrous, about as long as the stamens. Disk gland $0.5 \mathrm{~mm}$ high.
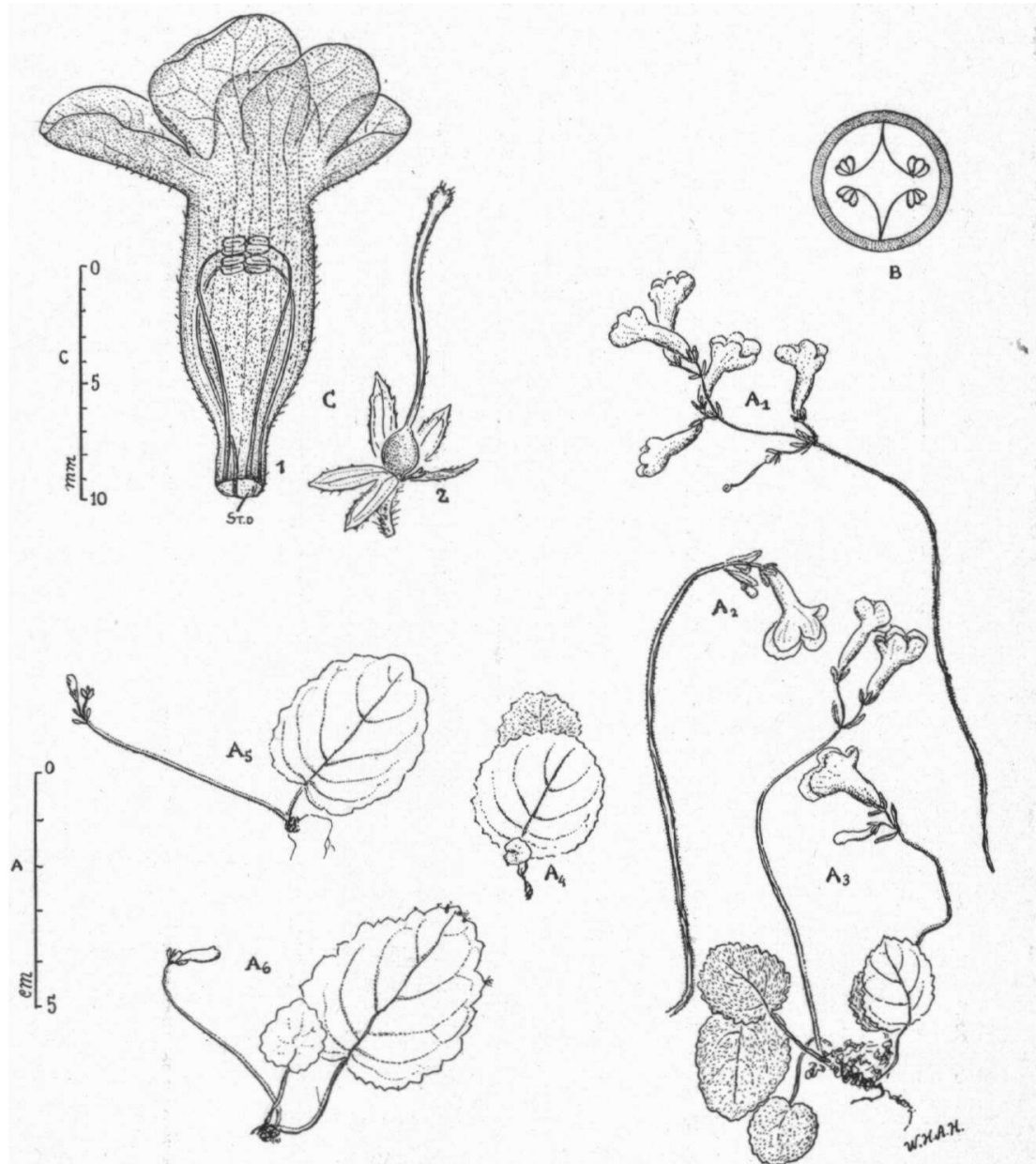

Fig. 31. Rhoogeton cyclophyllus: $\mathbf{A}_{1}$ and $\mathbf{A}_{\mathbf{2}}$. inflorescences; $\mathbf{A}_{\mathbf{3}-\mathbf{a}}$. plants; $\mathrm{B}$. diagrammatic transverse section of ovary; $C_{1}$. corolla; $C_{2}$. calyx with pistil ( $A_{2.6}$. . and $C_{1}$ : Maguire a.o. 40585; $A_{1.8,4}$ and $C_{2}$ : Jenman 896 ). 
Ecology: On dripping rocks, on montane slopes.

Britsh Gulana: Mt. Ayanganna, Pakaraima Mts., alt. 1000-1500 m, Maguire, Bagshaw \& Maguire 40585 (NY, holotype); Kaieteur, Jenman 896 (K).

2. R. viviparus Lwbg., p. 323.

Fig. 32, p. 431

Type: British GuiANa: Kaieteur, Jenman 880 (P, holotype, photographs: $\mathrm{U}, \mathrm{WAG}$, isotypes: $\mathrm{K}, \mathrm{U})$.

Leafblades ovate or oblong-ovate, acuminate at the apex, rounded or obliquely cuneate at the base, biserrate-dentate. Corolla red (?), 25-37 mm long; lobes subequal.

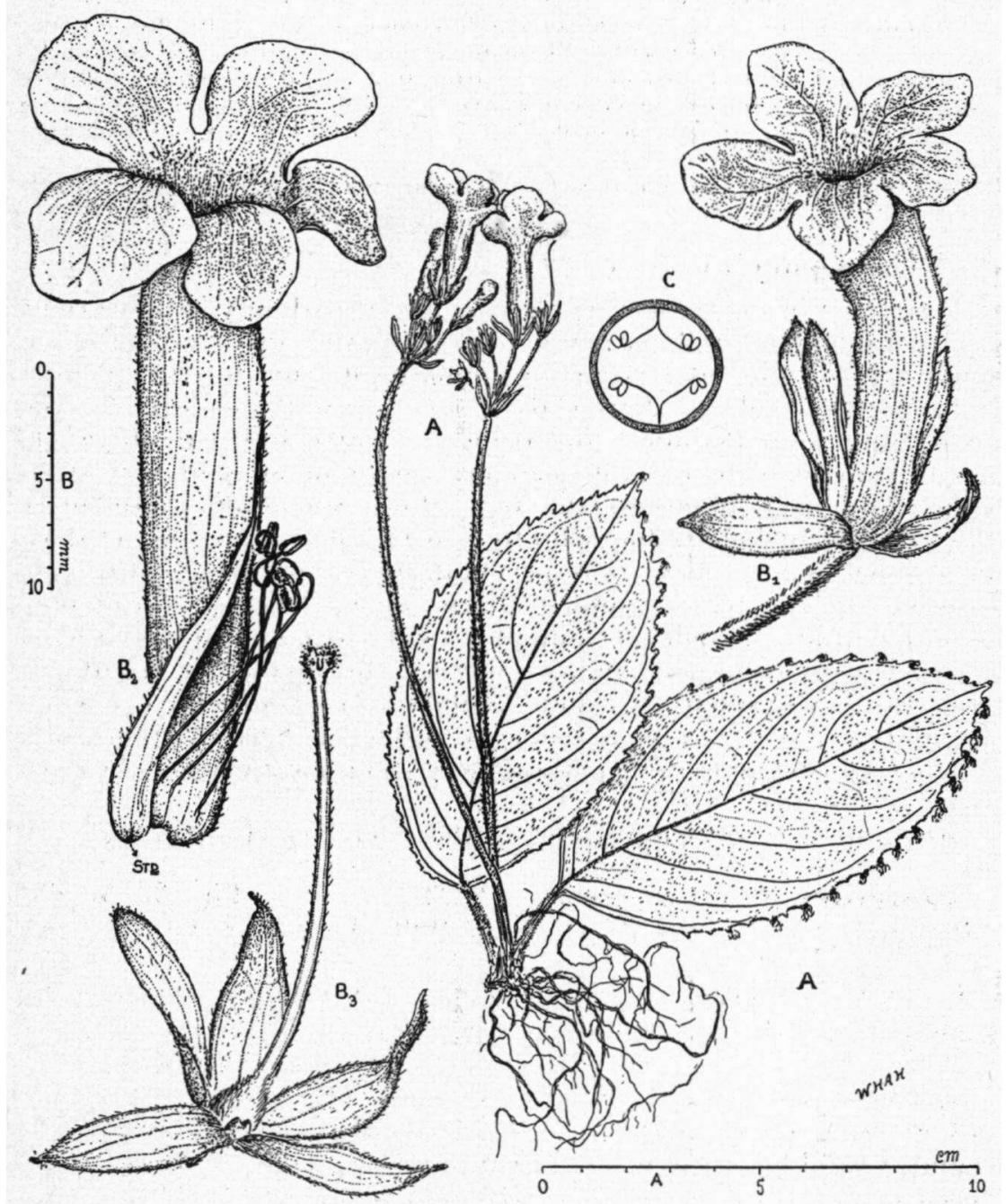

Fig. 32. Rhoogeton viviparus (Jenman 880): A. plant; $\mathbf{B}_{1}$. flower; $\mathbf{B}_{2}$. opened corolla; $B_{3}$. calyx with pistil; $C$. diagrammatic transverse section of ovary. 
Plant $10-30 \mathrm{~cm}$ high. Rhizome (?) with adventitious roots and some small lanceolate scales. Scales $5-6 \times$ ca. $1 \mathrm{~mm}$, strigillose. Leaves: petiole strigillose, $7-75 \mathrm{~mm}$ long; blade dark green above, paler beneath, about $2 \times$ as long as wide, $5.5-10.5 \times 2.5-5.5 \mathrm{~cm}$ or sometimes smaller, papyraceous when dry, sparsely strigillose to glabrous above, strigillose beneath, especially on the midrib and veins. Inflorescence umbellate or subcymose, 1-6-flowered. Peduncle $1-1 \frac{1}{2} \times$ as long as the leaves, sparsely strigillose at the base, glabrescent towards the apex, 7-22.5 cm long. Bracts lanceolate, 4-5 $\times$ as long as wide, 7-15 $\times 1.5-3 \mathrm{~mm}$, acuminate, entire, sparsely strigillose above, strigillose beneath. Pedicels strigillose, 5-20 mm long, with small bracteoles which are slightly smaller than the bracts. Sepals oblong, entire or with some teeth, sparsely strigillose on both sides, four subequal, 3-4 $\times$ as long as wide, $6-10 \times 1.5-3 \mathrm{~mm}$, the fifth (dorsal) somewhat smaller, curved around the spur. Corolla $3-4 \times$ as long as the calyx, at the base $2-3 \mathrm{~mm}$, at the throat 5-7 $\mathrm{mm}$ wide, sparsely strigillose outside; tube 18-28 $\mathrm{mm}$ long; limb 12-25 $\mathrm{mm}$ wide; lobes 5 , rounded; obscurely crenate-serrate, 4-10 $\mathrm{mm}$ in diam., two dorsal ones somewhat smaller. Filaments glabrous, contorted, inserted on the corolla, two lateral at $1 \mathrm{~mm}$ and two ventral at $2 \mathrm{~mm}$ from the base; anthers nearly square, all coherent, coherent in pairs, or free (?). Staminode glabrous, $0.25 \mathrm{~mm}$ long. Ovary pubescent, ovoid, $3-4 \times 2-2.5 \mathrm{~mm}$; style sparsely pubescent. Disk gland about $0.5 \mathrm{~mm}$ high.

British GuiANA: Kaieteur, on rocks near the waterfall, Jenman 880 ( $P$, holotype, sotypes: $K, U), 888(K)$.

\section{Tylosperma Lwbg., p. 323.}

Terrestrial herb. Stems very short, covered with adventitious roots. Leaves radicular, rosulate, petiolate or subsessile; blade oblanceolate, acuminate at the apex, short- or long-decurrent into the petiole. Inflorescence axillary, shorter than the leaves, paniculate. Flowers small, numerous. Peduncle and pedicels slender and without bracts. Sepals connate at the base, linear-lanceolate, long-acuminate. Corolla white, tubular, gibbous at the base; limb spreading. Stamens 4, didynamous, included; filaments free from each other, inserted on the corolla near the base; anthers reniform, coherent in pairs; cells divergent at the base, confluent at the apex, dehiscent throughout by a longitudinal split. Staminode small, without anther. Ovary superior; style included; stigma capitate. Disk glands 5, one dorsal large, the others small. Placentae ovuliferous on both surfaces. Fruit a capsule, 2- or 4(?)-valved; valves translucent. Seeds shining, subglobose, somewhat flattened, pustulate, obliquely mucronate at the base.

Distribution: Only one species in the Guayana Highlands.

T. cuneatum (Gleason) Lwbg., p. 323.

Fig. 33, p. 433

Basionym: Episcia cuneata Gleason, Bull. Torr. Bot. Cl. 58: 467. 1931.

Type: Venezuela: Amazonas, slopes of Mt. Duida, waterfalls at Aguita, Tate 879 (NY, holotype, photographs: U, WAG, isotypes: $\mathrm{K}$, US).

Leafblade 3-9 (mostly 5-6) $\times$ as long as wide, sharp-serrate, subtomentose to glabrous on both sides. Inflorescence practically secund. Corolla 8-10 mm long; limb 5-7 mm wide.

Plant 10-30 cm high. Leaves: petiole sometimes purple, up to $9 \mathrm{~cm}$ long; blade dark green above, paler beneath, 2.5-28 $\times 0.8-5 \mathrm{~cm}$, membranaceous or papyraceous when dry. Inflorescence: peduncle sparsely strigillose, 1-10 cm long. Pedicels 
sparsely strigillose, $0.5-3.5 \mathrm{~cm}$ long. Sepals green, subequal, $6-8 \times$ as long as wide, 3-6 $\times 0.5-1 \mathrm{~mm}$, slightly carinate, entire, sparsely strigillose outside, glabrous inside. Corolla erect in the calyx, $2-3 \times$ as long as the calyx, at the base $1-1.5 \mathrm{~mm}_{1}$ at the throat $1.5-2 \mathrm{~mm}$ wide, slightly contracted above the gibbosity, not so in the throat, not ventricose, glabrous outside; tube nearly cylindric, glabrous inside, 6.5-7.5 mm long; lobes subequal, ovate-oblong, 2-3 $\times 1.5-2 \mathrm{~mm}$, entire, with small glandular hairs above. Filaments glabrous, inserted on the corolla at about $2 \mathrm{~mm}$ from the base. Staminode glabrous, $0.5 \mathrm{~mm}$ long. Ovary practically glabrous or puberulous, ovoid, $2 \times 1.5 \mathrm{~mm}$; style erect, about as long as the stamens, 2.5-3 mm long. Disk glands: the dorsal one tongue-shaped, $1.2 \mathrm{~mm}$ long, longitudinally cleft when the capsule is mature, the others nearly terete, $0.6 \mathrm{~mm}$ long. Capsule glabrous or puberulous, oblong-ovoid, about as long as the calyx, $3-5 \times 2 \mathrm{~mm}$. Seeds dark brown, $0.3-0.4 \mathrm{~mm}$ in diam.

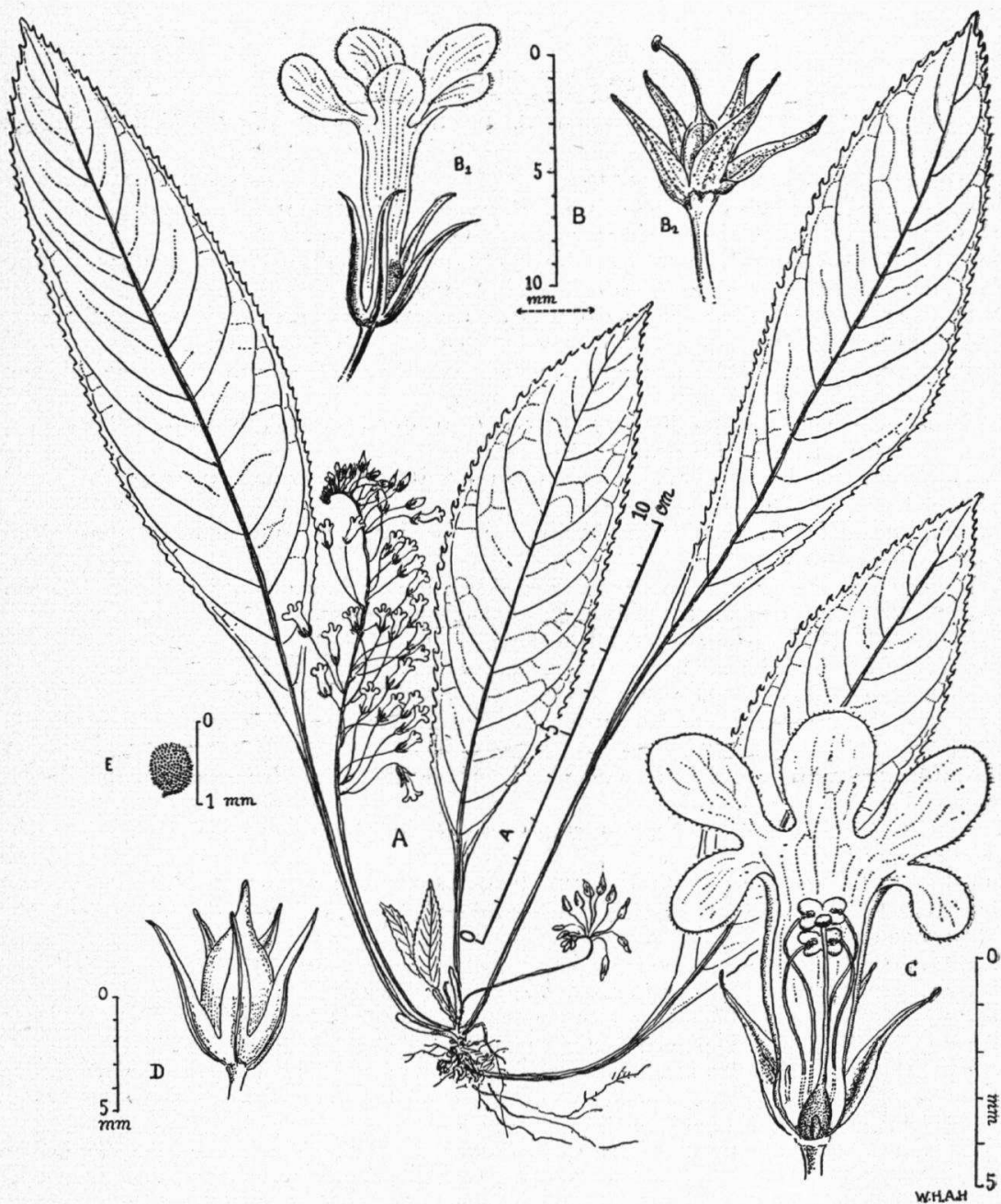

Fig. 33. Tylosperma cuneatum (Tate 879): A. plant; $\mathbf{B}_{1}$. flower; $\mathbf{B}_{2}$. immature fruit; C. opened flower; D. capsule; E. seed. 
Ecology: Among mosses on steep moist rocks near waterfalls, in high mixed forests (testibus Maguire \& Fanshawe), at middle elevations. Brutish Guiana: Makreba Falls, Kurupung R., Altson 357 (NY); ibid., Pinkus 11 (NY, US); Potaro R. Gorge, Maguire \& Fanshawe 23520 (NY, BR, F, G, $\mathrm{K}$, MO, U, UC, US).

\section{ACKNOWLEDGEMENTS}

The author expresses his gratitude to Prof. J. Lanjouw, under whose guidance the present work was undertaken, to Dr. F. P. JonkER, who suggested the working up of the Gesneriaceae for the Flora of Suriname, to Mr. G. V. Morton, who during his sojourn at Utrecht introduced him to the special problems of the family and who kindly helped and advised him in the course of his work.

\section{IMPORTANT LITERATURE}

Aublet, F. 1775. Histoire des plantes de la Guyane françoise 2 and 4. LondonParis.

Benthay, G. and J. D. Hooxer. 1876. Genera Plantarum 2, Gesneraceae. London. Burmann, J. 1756. Plant. Amer. Car. Plumier., Besleria and Columnea. Leiden. De Candolle, A. P. 1839. Prodromus 7, Gesneriaceae. Paris.

DoN, G. 1838. General History of the Dichlamydeous Plants, arranged according to the natural system 4, Gesneriaceae. London.

Duss, Père A. 1897. Flore phanérogamique des Antilles françaises, Annales Instit. Col. Marseille 4me année. 3me vol. ("1896"): 430-433, Gesnériacées.

Fritsch, K. 1894 ("1895"). Gesneriaceae, in A. Engler and K. Prantl, Die Natürliche Pflanzenfamilien 4(3b). Leipzig.

FrITsGH, K. 1906. Zweiter Beitrag zur Kenntnis der Gesneriaceen-Flora Brasiliens, in A. ENGLer, Bot. Jahrb. 37: 481-502.

Graham, E. H. 1934. Flora of the Kartabo Region, Annals Carneg. Mus. 22 ("1933"): 250-251, Gesneriaceae.

GrISEBACH, A. H. R. 1859-1864. Flora of the British West Indian Islands 459-465 1861 , Gesneriaceae.

Hanstein, J. 1853. Die Gesneriaceen des Königlichen Herbariums und der Gärten zu Berlin, 1, Linnaea 26: 145-216, t. 1-2.

HaNSTEIN, J. 1854. Idem 2 (1), Linnaea 27: 693-785.

HANSTEIN, J. 1857-1858. Idem 2 (2), Linnaea 29: 497-592.

Hanstein, J. 1865-1866. Idem 2 (3), Linnaea 34: 225-462.

Hanstein, J. 1864. Gesneraceae, in K. F. P. von Martius, Flora Brasiliensis 8 (1). München.

Hrtchcock, A. S. and M. L. Green, 1929. Besleria, in Proposals British Botanists. London.

Kunth, K. S. 1817-1818. Nova Genera et Species Plantarum (ed. folio) 2. 1818, Gesnereae. Paris.

Lemé, A. 1953. Flore de la Guyane française 3, Gesneriaceae. Paris.

Martius, K. F. P. von. 1829. Nova Genera et Species Plantarum 3, Gesnereae. München.

MoOre, H. E. 1953. The Scarlet Episcias, Baileya 1: 46-48, 89-101.

MOORE, H. E. 1954. Chrysothemis, Baileya 2: 86-88.

MOORE, H. E. 1954. Cultivated Species of Episcia, Baileya 2: 69-75.

MOORE, H. E. 1955. Drymonia macrophylla, Baileya 3: 109-112.

Moore, H. E. 1957. African Violets, Gloxinias, and their relatives, New York.

Morton, C. V. 1938. Gesneriaceae, in P. C. Standley, Flora of Costa Rica, Field Mus. Publ. Bot. 18: 1137-1187.

Morton, C. V. 1939. A revision of Besleria, Contrib. U.S. Nat. Herb. 26: 395-474.

Morton, C. V. 1944. Taxonomic Studies of Tropical American Plants, Contrib. U.S. Nat. Herb. 29: 1-40, Gesneriaceae.

Morton, C. V. 1953. Gesneriaceae, in J. A. Steyermark and collaborators, Contributions to the Flora of Venezuela, Fieldiana 28: 520-534. 
Morton, C. V. 1955. Gesneriaceae, in Flora of Trinidad and Tobago 2 (5). Trinidad.

Oersted, A. S. 1858. Centralamericas Gesneraceer = Kong. Dansk. Vidensk. Selsk. Skrift. 5 (5): 75-152, t. 1-11. 1859.

Plumier, C. 1703. Nova Plantarum Americanarum Genera, Besleria and Columnea. Paris.

Pulle, A. A. 1906. An Enumeration of Vascular Plants known from Suriname, Gesneriaceae. Leiden.

SANDWITH, N. Y. 1931. Contributions to the Flora of Tropical America, Kew Bull. 1931 : 489-492, Gesneriaceae.

Schomburgk, Richard. 1848. Reisen in Britisch-Guiana 3, Versuch einer Fauna und Flora von Britisch-Guiana, Gesneriaceae. Leipzig.

Solereder, H. 1909. Zur Systematik einiger Gesneraceen-Gattungen, insbesondere der Gattung Napeanthus, Beih. Bot. Centralbl. 24 (2): 431-439.

Sprague, T. A. 1912. The Genus Nautilocalyx, Kew Bull. 1912: 85-90.

TrapP, A. 1956. Zur Morphologie und Entwicklungsgeschichte der Staubblätter sympetaler Blüten, in $W$. T.ROLL and $H$. von GuTrenBerg, Bot. Stud. 5: 63-79, Das Androeceum der Gesneriaceen. Jena.

URBAN, I: Symbolae Antillanae 2: 344-388, Enumeratio Gesneriacearum. Leipzig.

\section{INDEX OF EXSICCATAE}

The number in parentheses corresponds with the species-number of the following enumeration. Only numbered collections have been listed.

1a. Alloplectus coccineus (Aubl.) Mart. ex G. Don var. coccineus

1b. A. co. var. fusco-maculatus Lwbg.

2a. A. cristatus (L.) Mart. ex G. Don var. brevicalyx Morton

2b. A. cr. var. crenatus Morton

2c. A. cr. var. cristatus

2d. A. cr. var. epirotes Lwbg.

3. "A". dariensis Seem.

4. A. ichthyoderma Hanst.

5. A. meridensis Klotzsch ex Hanst.

6. A. pycnosuzygius (Donn. Sm.) Morton

7. A. savannarum Morton

8. Besleria flavo-virens Nees et Mart.

9. B. insolita Morton

10. B. lanceolata Urb.

11. B. laxiflora Benth.

12. B. lutea $L$.

13. B. minutiflora Fritsch

14. B. pansamalana Donn. Sm.

15. B. patrisii D.C.

16. B. penduliflora Fritsch

17. B. saxicola Morton

18. - B. verecunda Morton

19. "Centrosolenia" conferta Morton

20. " $C$ ". decurrens Morton

21. "C". lineata Morton

22. Chrysothemis dichroa Lwbg.

23. C. friedrichsthaliana Hanst.

24. C. pulchella (Donn ex Sims) Dcne.

25. C. rupestris (Benth.) Lwbg.

26. C. villosa (Benth.) Lwbg.

27. Codonanthe calcarata (Miq.) Hanst.
28. C. crassifolia (Focke) Morton

29. Columnea affinis Morton

30. C. aureonitens Hook.

31. C. calotricha Donn. Sm.

32. C. guianensis Morton

33. C. lehmannii Mansf.

34. C. oerstediana Klotzsch ex Oerst.

35. C. scandens $L$.

36. Diastema hispidum (D.C.) Fritsch

37. Drymonia calcarata Mart.

38. D. campostyla Lwbg.

39. D. lanceolata (Hanst.) Morton

40. D. serrulata (Jacq.) Mart. ex D.C.

41. D. turrialvae Hanst.

42. Episcia bryogeton Lwbg.

43. . E. ciliosa (Mart.) Hanst.

44. E. congesta (Oerst.) Hanst.

45a. E. cupreata (Hook.) Hanst. var. cupreata

45b. E.c. var. viridifolia (Hook.)

Nichols.

46.' E. decumbens Mart.

47. E. densa C. H. Wright

48. E. fimbriata Fritsch ex descr., see p. 816 .

49. 'E. 'hirsuta (Benth.) Hanst.

50. E. inclinata Brandegee

51. E. lilacina Hanst.

52. E. longipetiolata Donn. Sm.

53. E. maculata Hook. $f$.

54. E. melittifolia (L.) Mart.

55. E. mimuloides Benth.

56. E. peltata Morton

57. E. porphyrotricha Lwbg.

58. E. punctata (Lindl.) Hanst. 
59. “E”. purpusii Brandegee

60. E. reptans Mart.

61. E. silvatica Cuatrec.

62. E. sphalera Lwbg.

63. E. urticifolia Lwbg.

64. Gloxinia perennis (L.) Fritsch

65. Hypocyrta nummularia Hanst.

66. Kohleria hirsuta (H.B.K.) Regel

67. Lembocarpus amoenus Lwbg.

68. Napeanthus andinus Rusby

69. N. apodemus Donn. Sm.

70. N. bracteatus Morton

71. N. jelskii Fritsch

72. N. primulifolius (Raddi) Sandwith

73. N. primulinus (Karst.) Benth. et Hook. ex Jackson

74. N. reitzii (L. B. Smith) Burtt

75. N. rigidus Rusby

76. N. riparius Philipson

Abbengets, N. J., 64 (49).

Abraham, A. A., 345 (17).

Allard, H. A., 20925, 22103, 22104, 22118 (64).

Allart, A., 152 (66), 478 (29).

Allen, P. H., 629 (4), 2674 (23), 2717 (21), 3528 (4), 3557 (40), 3579 (51, distrib. as $E$. fendleri), 3652 (24, distrib. as Tussacia woodsoni), 4594 (23), 4987 (39), 4996 (6), 5588 (51), Alston, A. H. G.; 7611 (45b)

Altson, R. A., 110 (80), 311 (17), 321 (49), $357(88), 371(42), 422,484(80)$.

André, E., 47 (12), 940 (45b), 2484 (28).

Appun, C. F., 338 (80), 976 (49), 1581 (83), $1872(80), 2125(83)$.

Archer, W. A., 1698 (63), 1703, 2107, 2210 (23), 2321 (55), 2432 (53).

Arnoldo, Bro. M., 862 (12).

Badier, de, 66 (54).

Bailey, I. W., 110 (27), 181 (27, type of C. bipartita).

Bailey, L. H., 709 (12).

Bailey, L. H. \& E. Z. Bailey, 731 (66).

Baker, C. F., 99 (la).

Bang, M., 1729 (68), 1729a (75).

Bangham, W. N., 415, 460 (40), 604 (28).

Bartlett, A. W., 8600 (53), 8743 (9).

Beard, J. S., 448 (12).

Beard, P., 1041 (35), 1076 (12), 1241 (35), 1285 (12), $1363(54), 1428$ (2b).

Beddington, 33 (11), 35 (1a), 44 (27), 45 (la).

Bélanger, C. P., 176 (2c), 178 (54), $179,180,191$ (12), $193(10), 194$ (2c), 196 (54), 312 (10), 365 (24), 366 (12), 776 (24).

Bellard, E. P. de, 18 (66).
77. "N". saxicola Brandegee

78. N. subacaulis (Griseb.) Benth. et Hook. ex Kuntze

79.' 'Nautilocalyx pallidus (Sprague) Sprague

80. N. pictus. (Hook.) Sprague

81. N. villosus (Kunth et Bouche) Sprague

82. Phinaea repens (Donn. Sm.) Solereder

83. Rechsteineria incarnata (Aubl.) Lwbg.

84. R. schomburgkiana (Kunth et Bouché) Kuntze

85. Rhoogeton cyclophyllus Lwbg.

86. R. viviparus Lwbg.

87. Tetranema mexicanum Benth: (Scroph.)

88. Tylosperma cuneatum (Gleason) Lwbg.

Benoist, R., 57 (27), 194 (28), 196 (1a), $497(38)$.

Bernouilli, G. and R. Cario, 2014 (40).

Berthoud-Coulon, 478 (40).

Biolley, P.; 6958 (40).

Blanchet, J. S., 1465, 1835, 1839 (1a), 1857 (8), 2121, 2122 (1a).

Boldingh, I., 1782B (12), 3825 (28), $3918 \mathrm{e}$ (1b).

Bond, F. E., T. S. Gillin and S. Brown, 110 (35), 219 (28).

Bowie, J. and A. Cunningham 19 (72).

Box, H. E., 1725 (2c).

Britton, N. L., 2553 (24).

Britton, N. L., and E. G. Britton, 2835 (28), 9185 (64).

Britton, N. L., E. G. Britton and T. E. Hazen, 395 (24).

Britton, N. L., and W. E. Broadway, 2811 (40).

Britton, N. L. and J. F. Cowell, 291 (12), $300(2 a)$.

Britton, N. L., W. G. Freeman and

L. H. Bailey, 2247 (78).

Britton, N. L., W. G. Freeman and W. Nowell $2541(28)$.

Britton, N. L. and T. E. Hazen, 252 (78), 1633 (28).

Britton, N. L., T. E. Hazen and W. Mendelson, 1277 (78), 1304 (66), 1784 (28).

Britton, N. L. and W. Mendelson, 823 (28).

Broadway, W. E., 1877 (12), 2291, 3132 (78), 3719 (2b), 4282 (55), $4525(54), 4526$ (2b), 5766 (40), $5789(35), 6172(24), 6175$ (28), 6738 (78), $6784 .(28), 6925$ (24), $6926,7486,7974$ (28), 9053 (40), 9856 (35). 
Buchtien; O., 1337, 1921 (68).

Burchell, W. J., 6455 (40), 9737 (27).

B.W. (Boschwezen $=$ Forest Dept. Suriname), $626(31), 645(11), 664$ (31), $1063(28), 2180(40), 2987$ (lb), 3486 (27), 3489 (28), 4115 (1b), 4598 (40), 4602 (24), 5642 (38), 5653 (1b), 5705 (11), 5723 (31), 5807 (67), 6328 (1b), 6619 (31), 6981 (47), 7033 (83), 7077 (67), 7103 (1b), 7226 (25).

Caley, G., 3 (40), 9 (54).

Chaffanjon, J., 330 (22).

Chickering, A. M., 111 (11).

Choussy, F., 48 (40).

Collenette, C. L., 463 (51, distrib. as E. chontalensis), 465 (24).

Cook, O. F. and R. F. Griggs, 614 (70).

Cooper, J. J., 5884 (34).

Cowan, R. S., 38247 (9), 38258 (1a), 38295 (34), 38310 (la), 38327 (27), 38361,38393 (1a), 38453 (9), 38513 (1a), 38555 (27), 38562 (45a), 38607, 38630 (37), 38725 (47), 38734 (1a), 38756 (34), 38776 (9), 38985 (1a), 39228 (47), 39337 (53), 39358 (27).

Cowan, R. S. and J. C. Lindeman, 39049,39179 (1b), 39219 (80).

Cowan, R. S. and B. Maguire, 38055 (27), 38072 (1a), 38137 (34).

Cruz, J. S. de la, 1135, 1316 (27), $1405(28), 1518,1535$ (53), $1584(80)$, 1588, 1787 (60), 2139 (47), 2197 (28), 2294 (27), 2327 (80), 2350 (60), 2810 $(28), 3022,3124(53), 3250$ (28), 3735 (27), 4395 (53), 4593 (24).

Cuatrecasas, J., 2046 (61), 13722 (23).

Cufodontis, G., 721 (28).

Dahlgren, B. E. and E. Sella, 520 (1a).

Davenport, 7 (57), 8 (24).

Davidson, M. E., 207 (4), 828 (40).

Dawe, M. 'T., 225 (45b), 256 (64), $281^{2}(45 b)$.

Dodge, C. W., 3469 (23).

Dodge, C. W. and V. F. Goerger, 9569 (28), 10153 (51, distrib. as Achimenes sp.), 10308 (11).

Dunlap, V. C., 352 (23).

Dusén, P., 3693 (74).

Duss, Père A., 178 (12), 179 (54), 328 (24), 1876 (35), 2377 (54), 2985 (2a), 3384 (24), 4637 (35).

Edwards, J. B., 396, 670 (40).

Eggers, H. F. A., 60 (54), 349b, 393 (24) $514(12), 602(2 \mathrm{a}), 613(54), 716(35)$, $835(54), 3524$ (12), 5985 (54), 6031 (12), 6160 (2b), 6199 (35, distrib. as C. speciosa), $6663(54), 6698$ (35, distrib. as C. speciosa), 6738 (12), 13078 (p.p.: 66 ; p.p.: 83), 13358 (66), $13525(40)$.

Ekman, E. L., 7271 (12).

Elias, Bro., 145 (64), 781 (45a).

Endrès, 178 (65), 184 (40).

Englesing, F. C., 154, 284 (11).

Erlanson, C. O., 493, 574 (23).

Ewan, J., 17081 (35).

F.D. (Forest Dept. Brit. . Guiana), 2022 (55), 2988 (47), 3024 (17), 3521 (53), 4158 (27), 6334 (18), 6897 (53), 7115 (47), "W.B. (Fr. G. WilsonBrowne S. J.) 70 (83), W.B., 569 (26), W.B., 623 (66), W.B., 635 (26).

Fendler, A., $178(28), 301$ (40). 322 (51, type of $E$. fendleri), 325 (11), 490 (28), 506 (78), 509 (24), 510, 513 (40), 770 (73), 790, 791 (30), 792, 793 (66), 795 (83), 2031 (35), 2033 (40).

Fernández, A., 325 (23).

Fernández, F., 99 (29).

Finlay, K., 178 (64), 179, 180 (40).

Florschütz, J. and P. A., 359 (lb), $515(28), 613,1181(27), 1326,1400$ (25), 1522 (28), 2213 (24).

Focke, H. C., 766 (40), 822 (64), 941 (27), 975 (1b).

Friedrichsthal, E. von, 1252 (23).

Friend, E. A., 128 (24).

Funck, N. and L. Schlim, 37 (12), 38 (2a) $152,242(66), 656(83), 1417$ (45a), 1511 (5), 1517 (83), 1699 (4).

Gaillard, A., 12 (22).

Galeotti, H., 1905 (87), 1922 (44).

Garcia-Barriga, H., 11528 (23).

Gardner, G., 581 (72, type of $\mathcal{N}$. brasiliensis).

Geay, F., 334 (1a).

Gentle, P. H., 1693, 2993 (28), 3016 (40).

Ginzberger, $A$. and M. Hagmann, 924 (27).

Glaziou, A., 1075, 8835, 18412, 19745 (72, distrib. as $\mathcal{N}$. brasiliensis).

Gleason, H. A., 58, 83 (47), 199 (80), 361 (27), 420 (17), 611 (80), 659, 660,661 (47), $664(53), 745,854$ (27). Goudot, J. (all numbers p.p.), 1, 2 (40), $5(61), 6(83)$.

Graf, L. J., 2 (35).

Graham, E. H., 397 (55), 513 (24).

Guillemin, J. B. A., 620 (72).

Gwyn Vaughan, D. J., 2 (27).

Hahn, L., 10 (10), 116 (12), 117 (54, distrib. as Besleria violacea), 118 (2c), 287 (35), 314 (10), 495 (24), 496 (54, distrib. as Besleria violacea), 512, 612, 1420 (10). 
Harlow, J. R., 53 (40).

Harris, W., 6313 (12), 9829 (64), 12101 (24).

Hartweg, K. T., 1103 (58), 1259 (83, type of $\boldsymbol{R}$. (Gesneria) stachydifolia).

Hatschbach, G., 1717 (74, distrib. as Hatschbachia reitzii).

Haught, O., 1846 (40), 2523 (76); $2574(30), 4345$ (66), 3062 (40), $4647,4726,4824$ (23).

Hayes, S., 23 (40), 188 (24).

Heyde, E. T. and E. Lux, $4034(40)$.

Hinton, G. B., 10582, 10616 (40, distrib. as $D$. grandiflora).

Hitchcock, A. S., 16948, 17234 (27), 17375 (17), 17386 (47), 17397 (53), 17595 (55), 17601 (53).

Hodge, W. H., 825 (2a), 828 (54), 6976 (23).

Hodge, W. H. and B. T., 1075 (35), 2710 (Pl. Exiscc. Gray. 1091) (54).

Holm, R. W. and H. H. Iltis, 14, 95 (11), 252 (23), 318 (51), 703 (40, distrib. as $D$. rubra var.), 816 (23).

Holt, E. G. and E. R. Blake, 817 (22).

Holton, I. F., 609 (40).

Hoskin, I., 22 (12), 50 (35), 100 (2a).

Hostmann, F. W., 208 (40), 285 (28), $570(40), 1225$ (lb).

Hostmann, F. W. and A. Kappler, 1372 (1b).

Howard, R. A:, 10345 (24), 11517, 11674 (35).

Hulk, J. F.; 243 (25), $416^{\circ}(27)$.

Humbert, H., 26630 (45).

Humboldt, F. W. H. A. von and A. J. A. Bonpland, $330(66), 2028$ (40, type of D. spectabilis), 2032 (83).

Hunter, A. A. and P. H. Allen, 329 (11).

Idrobo, J. M. and R. E. Schultes, 1337 (64).

Imray, J., 75, 152 (2a), 194 (35), 293 (12).

Jenman, G. S., 236 (27), 880, 888 (86), 896 (85), $1067(27), 1250(49), 1297$ $(80), 1908 .(83), 1939,2002$ (53), $2080(80), 2097 .(53), 2364$ (80), 2414 (47), 2419 .(53), 2517 (28), 3990 (24), 4156 (18), 5457 (80), 5608 (24), 7123 (55), 7983 (28).

Johnston, I. M., 62 (40), 141 (23).

Johnston, J. R., 278 (40).

Jonker, A. M. E. and F. P., 308, 314 (28), 486 (1a), 625 (38).

Jurgensen, C., 650 (44).

Kalbreyer, W.; 1048: (83).

Kappler, A., 2044 (62, distrib. as Tussacia villosa).
Kegel, H., 805 (28), 827 (40), 1370 (1b), 1430 (64).

Kenoyer, L. A., 536 (23).

Killip, E. P., 5077 (23), 5221 (28), 7884 (33), 11436, 35079, 35280, 35535,40038 (23).

Killip, E. P. and H. Garcia, 33232 (23).

Killip, E. P. and T. Lasser, 37773 (29).

Killip, E. P. and A. C. Smith, 18349 (4), 19412 (66), 19851 (5), 20033 (4), 23374, 23393, 24076, 25207 (64).

Klug, G., 1836, 2232 (40), 2855, 2877 (48), 3998, $4214(64)$.

Køie, M., 4766 (23).

Krukoff, B. A., 1217, 1353 (1a), 1405 (48), 1422 (37), 1526 (1a), 5890a (79), 6161 (37), 6245,6516 (27).

Kuntze, O., herb. 624 (24), 831 (78), 1383 (66).

Kupper, W., 454 (69).

Kuyper, J., 1(28).

Lane, M., 444 (12).

Lang, $H$. and A. C. Persaud, 155 (80).

Langlassé, E., 81 (83).

Lanjouw, J., 1244 (27).

Lanjouw, $J$. and J. C. Lindeman, 1410, 1940 (28), 2096 (1b), 2104 (28), 2373 (8), 2466, 2593 (67), 2686 (80), 2742 (31), 2833 (67), 2890 (80), 2921 (31), 2941 (80).

Lansberge, J. C. van, 313 (30).

Leblond, J. B., 16 (12), 284 (1a), 286 (54), 439 (12)

Lehmann, F. C., 2631, 4717 (24), 6063 (33), 8423 (23), 8865 (45b), CCI (60).

Liebmann, F., 9214 (44).

Lindeman, J. C., 3613 (28), 4987 (1b), $5186(28), 6814(40)$.

Linden, J., 42 (66); 236 (30), 981; 1408 (83).

Lloyd, F. E., 184 (12), 191 (2a).

Lundell, C. L., 441, 6549 (40).

Macbride, J. F., 4658 (64).

Maguire, B., 23935 (40), 24539 (8), 24762 (25), 40488 (55), 40702 (lb), 40788, 40806 (67), 40808 (80).

Maguire, B., W. M. C. Bagshaw and C. K. Maguire, 40585 (85), 40586, 40588 (49), 40594 (2d), 40595 . (7).

Maguire, B. and D. B. Fanshawe, 22826 (53), 22838 (27), 22982 (47), 23019 (43), 23036 (47), 23047 -(80), 23067 (32), 23078 (49), 23127 (7), 23520 (88), 32368 (43), 32392 (42).

Maguire, B. and G. Stahel, 22765 (28), 25050 (27)

March, W. T.; 1185, 1333 (24). 
Martin, J., 100 (pp. 34; p.p. 35), 274 (80), 275 (31), 276 (8).

Martinez-Calderon, G., 50 (40).

Martius, K. F. P. von, 3014 (37), 3091 (60), 3117 (43), 3119 (46), 3239 (37).

Martyn, E. B., 53 (53), 226, 303 (80).

Matthews, A., 2010 (64).

Matuda, E., 3440 ( 40 , distrib. as $D$. chiabensis?) 4864 (58), 16429. (45), 16457 (50), $16572(40)$.

Maxon, W. R. and A. D. Harvey, $8011^{\prime}(41)$.

Mélinon, M., 58 (71), 109, 342, 434 (1a).

Mell, C. D. and R. C., 200 (80).

Mennega, A. M. W., 378 (27), 482 (83), 560, 564 (28).

Metcalf, R. D. and J. Cuatrecasas, 30098 (56), 30200 (19).

Mexia, Y., 6271a (79, distrib. as Alloplectus), 7182 (64), 8410, 8410a (33, distrib. as Alloplectus).

Meyers,'J. G., 5817 (27), 5827 (1a), 5839 (11).

Miers, J., 4231 (72).

Miller, G. S., 127 (35).

Monteiro da Costa, R. C., 225 (1a).

Moore, S., 529 (40, type of D. maculata).

Morton, C. V., 4618, 5291, 5532, $6224(35)$

Morton, C. V. and J. Acuña, 3329 (12).

Moritz, J. W. K., 413, 414 (66), 870 (73), $1127(66), 1132$ (5).

Mouret, F., 218 (35), 219 (2c), 222 (12).

Nurse, H. W., 6857 (40).

Oersted, A. S., 9291 (34).

Orcutt, C. R., 2345 (24).

Othmer, B., 217 (78), 455 (24).

Paul, Bro., 134 (23).

Pearce, R., 226, 543 (64).

Peck, M. E., 513 (40).

Pennell, F. W., 1846 (64), 4588 (23).

Pennell, F. W. and E. P. Killip, 8329 (83).

Pennell, F. W., E. P. Killip and T. E. Hazen, 8620 (40).

Pérez Arbeláez, E. and J. Cuatrecasas, $6765(60)$.

Perrottet, G. S., 262 (12), 266 (54).

Persaud, A. C., 350 (80).

Philipson, W. R. and J. M. Idrobo, 1958 (45b).

Philipson, W. R.; J. M. Idrobo and Fernández, 1679 (76).

Philipson, W. R., J. M. Idrobo and Jaramillo, 2051 (76).

Pinkus, A. S., 11 (88), 12 (42), 13 (49), 244 (27).

Pittier, E., 104 (66).
Pittier, H., 466, 467, 2488 (23), 2493 (52), 2550 (40), 2567 (51), 3490 (23), 3843,4017 (40), 4085 (23), 5111, 5200 (24), 7336 (51), 7894 (83), $9864(66), 10130$ (29), 11251 (66), 11930 (81), 12173 (29), 12218, 13057 (64, p.p.?), 13140 (66), 13521 (35), 13879 (29), 16008 (69), 16009 (51).

Plée, A., 471 (35).

Poeppig, E., 1031 (36), 1619 (64, type of $G$. heterophylla).

Privault, D., 109 (54), 113 (2c), 149 (12), 198 (54).

Proctor Cooper, G., 142 (23), III 57 (54), III 258 (p.p. 23, p.p. 24).

Pulle, A. A., 134 (27), 437 (24), 444 (28), $492(83)$, H 13 (24).

Purdie, W., 177 (40, type of D. parvifolia Griseb.), 470 (45a).

Purpus, G. A., 6701 (77), 6850 (14, distrib. as Episcia aurea), 6851 (50), 7003 (11, type of $B$. chiapensis), 7008 (65, type of Episcia truncicola), 7216 (59), 7533 (14, type of Episcia aurea).

Quentin, R. P., 35 (54), 64 (12), 459 (54), 485, 700 (2a).

Reed, E., 699 (45b).

Rendle, A. B., 131 (12).

Richard, Mme., 46 (54).

Riedel, L., 419 (8), 1277 (64).

Riley, L. A. M., 301 (23).

Rodriguez, J. V., 3028 (45).

Rodriguez, L., 2593 (54), 2665 (12), 2774 (2a), 3358 (54), 3363 (2c), 3609 , 3806 (35), 3864 (12), 3940 (54), 4494 (12), 4707, 6712 (54).

Rohr, J. von, 9, 140 (54).

Rombouts, H. E., 119 (25), 158, 474, 505 (83), 790a (28), 828 (1b).

Rose, Mr. and Mrs. J. N., 21739 (66).

Rovirosa, J. N., 870 (11).

Rowlee, W. W. and H. E. Rowlee, 375 (11).

Sagot, P., 425 (1a), 426 (28, type of C. confusa), 899 (40).

Salvador Calderón, 1343 (64).

Salvoza, F. M., 843 (40).

Samuels, J. A., 97 (40).

Sandeman, C., 94 (64).

Sandwith, N.' Y., 3 (80, type of $\mathcal{N}$. lacteus), 49 (47), 58 (17), 286 (27), $316(28), 659$ (55), 1047 (80, distrib. as $\mathcal{N}$. lacteus), 1216 (27), 1279 (49), $1362(7), 1385(28), 1837(35), 1866$ (40), 1895 (24), 1902 (55), 1915, 1922 (78).

Schiefer, H., 787. (45b). 
Schipp, W. A., 1046 (40), S-639 (11). Schlim, L., 93 (66), 533 (45a), 958 (40), 1087 (4), 1668 (45a).

Schomburgk, R., 37 (57), 110 (1a), $118(84), 125$ (55), 205 (11), 843 (55).

Schultes, R. E. and M. Villarreal, 7394 (23).

Schulz, J. P., 7611 (25).

Seemann, B. C., 61 (11), 1058 (3).

Seibert, R. J., 361 (11), 556, 569 (23), $2240(64)$.

Shafer, J. A., 489, 546 (35).

Shank, P. J. and A. Molina, 4958 (23).

Sherring, R. V., 267 (2b).

Sieber, F. W., 14(54), 161 (2c), 308 (10), 319 (p.p. 10, p.p. 12), 408 (54), 409 (12).

Silva, A., 136 (37).

Simmonds, N. W., 207 (24), 226-(35), 274 (24), 301 (78).

Skutch, A. F., 2195, 2854 (41), 3553 (39), 3554 (34), 4854 (69), 4947 (11), 4951 (23).

Smith, A. C., 2123 (53), 2531 (1a), 2708,2815 (27), 2880 (1a), 2886 (11), 2887 (1a), 3026 (28), 3555 (11), 3579 (26), 3652 (84).

Smith, G. N., 72 (2b), 107 (p.p. 35, p.p. 2b).

Smith, H. H., 1395, 1396, (30) 1398 (83, distrib. as Gesneria stachydifolia), 1399 (11, type of $B$. debilis), 1402 (40, distrib. as $D$. spectabilis), 2672 (11, type of $B$. tenuifolia).

Smith, H. H. and E. W. Smith, $629(54)$.

Smith, J. D., 5109 (69), 6703 (41), $6707,6708,6709$ (69), 6717 (11), $6718(40), 6728$ (31).

Sneidern, K. von, 5189 (23).

Soukup, J., 2212 (64).

Sprague, T. A., 123 (45b).

Splitgerber, F. L., 102 (64), 586 (40), 716 (lb).

Spruce, R., 1850 (1a), 2407 (24), 2604 (1a), 2654 (7), 2801 (60), 3024, 3149 (83), 3379 (88), 3612 (22), 4513 (64).

Stahel, G., 180, 248 (227).

Standley, P. C., 7827 (11), 28842 (64), $33416,33515,33542$ (34), 36891, 36918 (69), $37740,38122,39163$, 39644 (34), 40952, 41121 (23), 52648 (11), 52699 (28), 52773, 52941, 53880, 54132,54572 (11), 71201, $90621 \mathrm{a}$, $90623(70)$.

Standley, P. C. and R. Torres, 51229, 51283, 51651,51779 (34).

Standley, P. C. and J. Valerio, 45390 (69), 47092 (41, distrib. as D. marmorata).

Stehlé, H., 141 (24), 381 (64), 5771 (54).

Stehlé, H. and H., 2133 (2c), 2172 (54).
Steyermark, J. A., 31356 (45a), 39935, 41687, 41978, 44881 (11), 45412 (28), 49247, 49636 (11), 55037 (66), 56023 (5), 56066 (45b), 56142 (30), 56347 (45a), 56469 (5), 57016 (73) 57145 (66), 57965 (30), 57974 (11), 57992 (88), 57996 (49, distrib. as $E$. cordata), 58089 (27), 59950 (16), $60582(66), 60732(28), 61008,61995$ $(30), 62140(40), 62199(30), 62229$ (35), 74739 (27), 75390 (28).

Steyermark, J. A. and P. H. Allen, 17254 (51, distrib. as Achimenes grandiflora).

Stoffers, A. L., 3234, 3438, 4233 (12).

Stork, H. E., 489 (34).

Tamaya, F., 1670 (29, distrib. as $C$. aureonitens).

Tate, G. H. H., 878 (49, type of $E$. cordata), 879, (88, distrib. as Episcia cuneata).

Tate, R., 314 (11), 317, 318, 319 (51, syntypes (?) of $E$. chontalensis).

Terrasson, 38 (12), 39 (2c).

Terry, M. E. and T. Terry, 1421 (41), 1471 (69).

Thiébaut, C., 7 (12), 331 (35), 375 (2a), 832 (54).

Thurn, E. im, 287 (16).

Tonduz, A., 6679 (11), 8902 (40), 9553 (69), 9559 (11), 10938 (34), 11511 (11), 13039 (34), 14719 (11).

Traill, J. H. W., 625a (27).

Tresling, J., 251 (40), 411 (80), 470 (25).

Triana, J., 473 (45b), 2486 (33), 2539 (23), 2557 (61), 4161 (45b), 4167 (23).

Trinidad, herb. (see also N. W. Simmonds), 2506 (78), 2514 (35), 3034 (64), 7008 (28), $13973=$ Simmonds $207^{(24)}, 14017=$ Simmonds 226 (35), 14139 (66), $14169=$ Simmonds 274 (24), 14729 (66), 15059 (28), $15061(66), 15298(35)$.

Tucker, J. M., 1345 (40).

Tuerckheim, H. von, 7643 (40), 7644 (11), 7647 (82, distrib. as Napeanthus repens), 7756 (11), 8542 (31), II 89 (82, distrib. as Napeanthus repens), II 114 (40), II 162 (11), II 810 (31), II 1740 , II 2281 (14).

Tutin, T. G., 84 (80), 217, 218 (47).

Ule, E., 2417 (72), 5215 (1 a), 5493 (64), 6211 (13), 8459 (11), 8751 (16), 8760 (32), 9774 (40).

Versteeg, G. M., 253 (1b), 329 (27), 342,354 (18), 394 (28), 760 (25). 
Wachenheim, 146 (1a), 162, 173 (38), 209 (62).

Ward, R, and Kortright, 8795 (40).

Weber, N. A., 347 (27).

Weberbauer, A., 2324 (64).

Wedel, H. von, 709 (11), 789 (23), 790 (51, distrib. as Achimenes grandiflora), 912, 1026 (11), 1130 (23), 1156 (28), 1219, 1272, 1281 (40), 1296 (28), 1388 (40), 1456 (51, distrib. as Achimenes grandiflora), 1458 (23), 1555 (40), 1597 (11), 1607 (51), 1646 (11), 1875 (28), 1890, 2006, 2052 (40), 2138 (11), 2306 (41), $2321,2340,2453$ (11), 2497 (40), 2509 (23), 2549 (28); 2621. (23), 2782, 2893 (28).

Went, F. A. F. G., 292 (40), 379 (27), 1020 (24).

Williams, Li., 10052 (35, distrib. as C. fendleri), 10455 (66), 11146 (83),
11460 (24), 12230 (35, distrib. as C. fendleri).

Williams, L. O., 16072 (34).

Williams, L. O. and A. Molina, 14439 (11).

Williams, R. S., 731 (11), 939 (23).

Woodson, R. E., P. H. Allen and R. J. Seibert, 1195 (24, type of Tussacia woodsoni), 1614, 1642 (23), 1765 (40), 1826 (11).

Woodson, R. E. and R. W. Schery, 878 (23), 905 (11).

Woodworth, R. H. and P. A. Vestal, $371(40)$.

Wullschlaegel, H. R., 379 (28), 380 (40), 767 (64), 1322 (1b).

Yuncker, T. G., 4718 (11).

Yuncker, T. G., J. M. Koepper and K. A. Wagner, 8001 (28), 8303 (11). 


\section{INDEX OF NAMES}

(New names or combinations are in bold face, synonyms in italics. Page numbers of principal entries in bold face.)

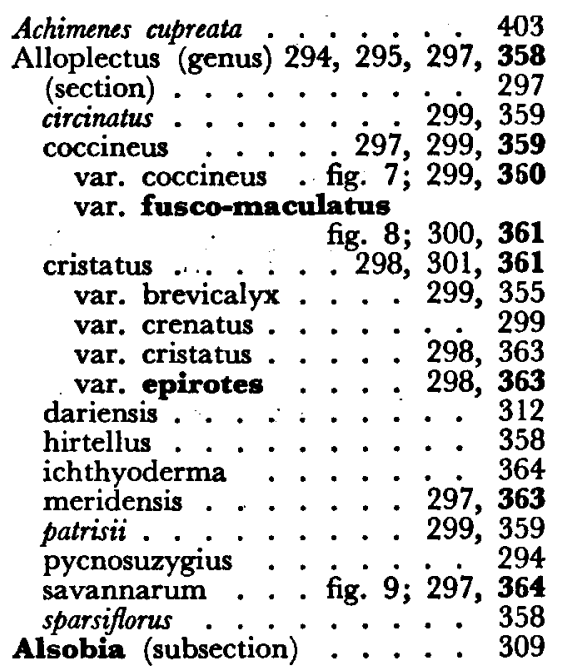

Besleria (genus) 294, 297, 300, 365 (section)....... 301 berteriana . . . . . 371 chiapensis : . : 369 coccinea . . . . 299, 359 cristata . . . 298, 300,361 debilis . . . . . 369 flavo-virens fig. $10 ; 300,301,366$ guadalupensis ...... 408 imrayi . . . . . . . . 371 incarnata . . . . 320, 425 insolita . . . . fig. $12 ; 368$ lanceolata . . . . 369 laxiflora . f fig. 11; 369 lutea . . 300,301, 355, 366, 371 luteo-virens . . . . 301, 367 melittifolia : . . 300, 335, 408 minutiflora . . . . . 301 pansamalana : . : 317 patrisii . . . . . fig. $12 ; 371$ penduliflora : . . 373 pulchella . . $329,334,375$ saxicola . . . . . fig. $12 ; 373$ serrulata . . . 306, 395 spectabilis . . . . . 306, 396 tenuifolia . . . . . . 369 verecunda . . . . fig. $12 ; 374$

Brachyloma hirsutum . . . 317, 417 rhodomallon ..... 317, 417

Centrosolenia (section) . . 308, 311
Centrosolenia (subsection) . . 310 aena ....... 316 conferta . . . . . 312 decurrens . . . . . 312 densa .......... 404 glabra . . . . 302, 381 hirsuta . . .... 310, 405 lineata ...... 312 picta . . . . 319, 422

Chirita lavandulacea . . . 355

Chrysothemis . . . . . 329, 375 aurantiaca . . . 335, 376 dichroa ... fig. $1 ; 329,331$ friedrichsthaliana fig. $1 ; 329$, 333. 355 melittifolia .... . 340, 408 pulchella $329,330,334,355,375$ rupestris figs. 2,$3 ; 329,336$, 355,376 semiclausa . . . . 329,338 venosa . . . . . 340, 408 villosa : fig. $3 ; 329,338,377$

Codonanthe . . . . 302, 378 bipartita . . . . . 379 calcarata fig. $13 ; 302,35 \dot{3}, 378,381$ confusa . . . . . 302, 381 crassifolia fig. $13 ; 302,355,381$ gracilis . . . . . . 378 Collandra (section) . . 294, 303 aureonitens ..... 355, 383 pilosa . . . . . . 383 picta . . . 319, 422

Columnea (genus) 294, 296, 303, 382 (section) . . . . . 303 affinis . . . . . . 385 aripoensis $\therefore 303,390$ aureonitens . . . 303, 383 calotricha . figs. 14,$15 ; 303,385$ ciliosa . . . . . . . 401 fendleri. . . 303, 304, 390 gloriosa . . . . 293, 355 guianensis . . fig. 16; 303,386 jacquiniana . . . 303, 390 lehmannii . . . . 295 oerstediana fig. 17; 303, 355, 388 picta. . . . . . 319, 423 pilosa : : : : . 383 rotundifolia : . 303, 390 sanguinea . . . . . . 340 scandens . . . 303, 382, 389 var, aripoensis . . 303, 304, 390 var. australis : . 303, 304, 390 var. vincentina : $303,304,390$ speciosa . . . 303, 390 


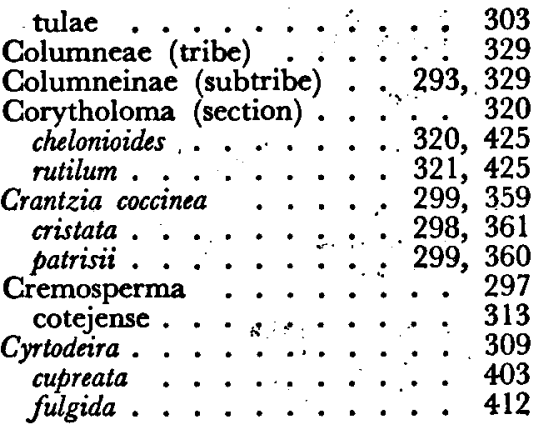

Diastema hispidum . $\because \because 317$

Drymonia (genus) 295, 296, 305, 391 (section) . . . . . 305 bicolor . . . 306,395 buscalionii . . 308, 392 calcarata . . fig. $18 ; 308,392$ campostyla figs. 19, 20;305, 393 cristata. . . . 306, 397 jacquini : : . 306, 396 lanceolata . . . . . 317 maculata . . 306, 397 marmorata . . . . . 398 parvifolia $: \ldots 306,397$ sarmentosula . . . 306,397 serrulata . f fig. $21 ; 306,355,395$ spectabilis . . . . 306, 396 turrialvae . . . . . . 398 villosa . .

Elongatae (subsection) . . . . 301 Episcia (genus) 295, 296, 297; 398 (section) . . . . . . 309 (subsection) . . . . . 309 aurea . . . . . . 317 bicolor : . . . 309 bryogeton : fig. $22 ; 312,400$ buchtienii . . . ..... 312 ciliosa $\because$ fig. $23 ; 312,401$ congesta . . . . . 312 cordata . . . 310, 405 cuneata . . . 317, 323, 432 cupreata . . . . 309, 403 var. cupreata .... 404 decumbens . . . . . . 311 densa . . . 313, 404 dianthiflora . . . . 309 elongata ... . . . . . . 317 erythropus . . . . . . 312 fimbriata . . . . . . 310 fulgida. . . : : 412 glabra . . . . . 312 guadalupensis . . . . . 408 hansteiniana. .312 hirsuta . fig. 24;308, 310, 405 hispida . . . . . 317 hookeri : .... 302, 317, 381

inclinata. . . 311

lanceolata . . . . . 317

lilacina ........ 309

longifolia . . . 316

longipetiolata . . . . . 312

luciani ..... . 316 maculata . . 312, 355, 407 melittifolia . . . 301, 311, 408 var. guadelupensis . . . 408 mimuloides fig. 25; 296, 311, 409 ochroleuca ....... 316 peltata . . . . . . 310 picta. . . . . . 319, 422 porphyrotricha fig. $26 ; 310$, pulchella ... . . 317, 335, 376 punctata . . . . . 309 purpusii . . . . . 317 reptans $\quad 308,309,399,412$ rosea ........ . 317 silvatica . . . 309 sphalera . . fig. $27 ; 310,413$ subacaulis. . . . . 317, 353 truncicola . . . . . . 317 urticifolia . . . 313, 314 villosa ....... . . 320, 424

Gentianaceae . . . . . 340

Genuinae . . . . . . . . 305

Gesneria chelonioides . . . 320, 425 elongata . . . . . . 317

guianensis ... . . . . . 427 hirsuta . . . . 317, 417 lindeniana . . $\because 321,425$ rutila . . . . . 321, 425 schomburgkiana . . . 427 stachydifolia. . . 321, 425

Gloxinia . . . . 297, 415 heterophylla . . . . 415 maculata . . . . . 415 perennis . $\because 355,415$ suaveolens : $\because \ldots \ldots . . .416$ trichantha . . . . . . 415 trichotoma $\because \because \because \because \because \because 415$

Glycanthes scandens : : 303,390

Hatschbachia . . . . . . 341 reitzii ...... 349

Heintzia (section) . . . . . 295

Hypocyrta . . . . . 294, 296 ciliosa . . . . . . . 401 crassifolia . . . . . 302, 381 nummularia . . . . . 317

Isoloma hirsutum . . . . 317, 417

Klugia . . . . . . . 324

Kohleria : : 317,416

Kohleria amabilis . . . 293, 355 hirsuta ..... 317, 417 rhodomallos . . . . . 317, 417 spicata ...... 355 


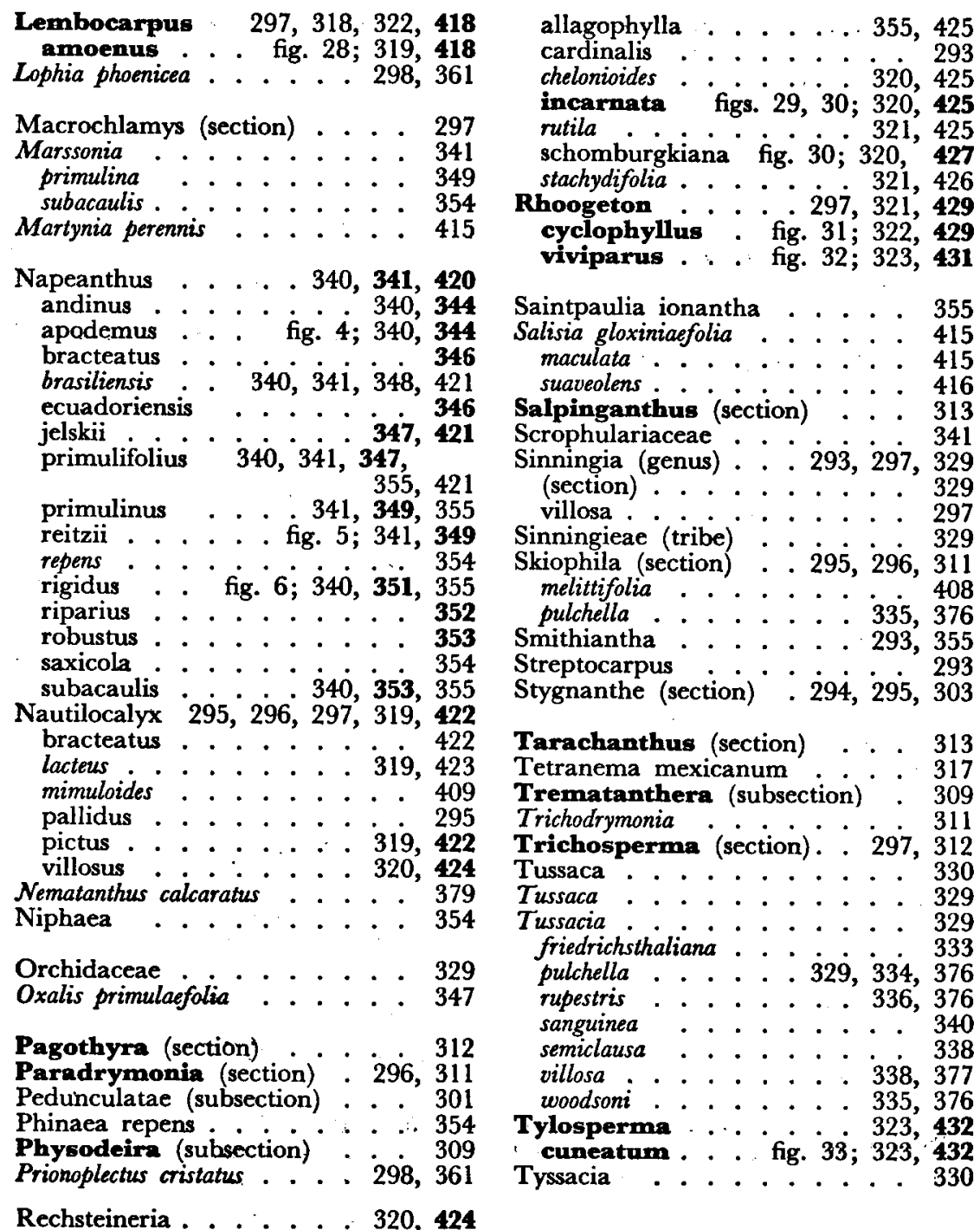

